\title{
Lower limb immobility and venous thromboembolism risk: investigating preventive measures.
}

By Irene Braithwaite $\mathrm{MBChB}$

A thesis submitted to the Victoria University of Wellington in fulfilment of the requirements for the degree of Doctor of Philosophy

Victoria University of Wellington

2016 
Dr Irene Braithwaite: Lower limb immobilisation and venous thromboembolism risk Copyright (C) 2016

\section{SUPERVISORS}

Professor Richard Beasley

Professor John Miller

Wellington 


\section{Abstract}

Prolonged work-related seated immobility and lower limb immobilisation (LLI) are two situations in which the risk of venous thromboembolism (VTE) is poorly understood, and no evidence-based guidelines for the reduction of risk exist.

The aim of this research was to investigate the role of prolonged work-related immobility and LLI as risk factors for VTE and to assess the haemodynamic effects and feasibility of two possible preventive measures; the Legflow device during prolonged work-related immobility and an intermittent pneumatic compression device (IPC) during LLI.

Four studies of work-related immobility were conducted: a case-control study to assess the risk of prolonged seated immobility and VTE; a meta-analysis of two case-control studies to assess the risk of VTE in sedentary workers; a study of popliteal vein haemodynamics in ten adults using the Legflow device while seated and a feasibility study of the Legflow device in 96 sedentary office workers in their working environment. Four studies of LLI were completed: a meta-analysis of two case-control studies to assess the association between LLI and VTE risk; an audit of VTE rates in patients with Achilles tendon injury undergoing LLI prescribed prophylactic aspirin; a study of the effect of an IPC device beneath a fibreglass cast on popliteal vein haemodynamics in 24 adults and a feasibility study of the IPC device in patients undergoing LLI at the Fracture Clinic at Wellington Regional Hospital.

There was no association between prolonged seated immobility and VTE (odds ratio (OR) $1.18,95 \% \mathrm{Cl} 0.56$ to $2.48, \mathrm{P}=0.67)$. Each additional hour seated in a 24 hour period was associated with VTE (OR $1.08,95 \% \mathrm{Cl} 1.01$ to $1.6, \mathrm{P}=0.02)$. There was an association between sedentary occupations and VTE (OR $1.79,95 \% \mathrm{Cl} 1.22$ to $2.63, \mathrm{P}=0.003)$. The Legflow device increased popliteal vein peak systolic velocity (PVPSV) (difference between the Legflowmobilised and immobile limb adjusted for baseline $60.0 \mathrm{~cm} / \mathrm{s},(95 \% \mathrm{Cl} 44.6$ to $75.3 \mathrm{P}<0.001)$. In the working environment $50 \%(95 \% \mathrm{Cl} 40$ to $60 \%)$ of sedentary office workers were adherent (use $\geq$ four times per day) with the Legflow device. 
LLI was associated with VTE (OR 73.1, 95\%Cl 10.1 to 530, P<0.001). A total of $14 / 218$ (6.4\%, $95 \% \mathrm{Cl} 3.6 \%$ to $10.5 \%)$ Achilles tendon patients prescribed aspirin developed VTE, an incidence similar to the $6.3 \%$ identified in a previous patient group not routinely prescribed VTE prophylaxis. The haemodynamic effect of the IPC device was not impaired by its placement within a fibreglass leg cast (difference in PVPSV between IPC in-cast and IPC outside-cast $-0.8 \mathrm{~cm} / \mathrm{s}, 95 \% \mathrm{Cl}-6.5$ to $4.9, \mathrm{P}=0.78)$. Only $7 / 142(5 \%, 95 \% \mathrm{Cl} 2.0$ to $9.9 \%)$ of potentially eligible patients utilised the IPC device in the feasibility study.

Prolonged work- and computer-related seated immobility and sedentary professions are associated with VTE. The Legflow increases venous blood flow in seated adults and is a feasible device for use in the office environment. The introduction of aspirin for VTE prophylaxis of VTE during LLI did not influence VTE rates. The haemodynamic effect of the IPC is not impaired in a fibreglass cast, but its use is not a feasible option in the clinical setting of LLI. 


\section{Acknowledgements}

Well, who would have thought I would write a PhD?

It seems quite a few people, without whom I am sure I never would have completed the thesis!

First and foremost, I would like to thank my main supervisor, Professor Richard Beasley. Without his constant enthusiasm, enduring positivity and unending support I am sure I would still be in the planning phase of this thesis.

Professor Mark Weatherall, has, I think, discovered a previously untapped well of patience during our statistical conversations, and I thank him for the rigor that he has demanded from myself, my studies and study data.

Professor John Miller, my VUW supervisor who has so capably pointed me in the right direction as and when required.

My husband Paul has looked out for me, put up with me, provided me with hot dinners, space to spread out references, chased the cat off my computer seat, plied me with wine when necessary and with exercise 'just because'. I hope he might be the first to call me 'Doctor Doctor'!

I have been lucky enough to have the most supportive colleagues at work, Janine Pilcher, Mathew Williams, Anthony Mallon, Mark Holliday (my ever so patient office buddy) and the Semprinis to name a few. Ali who does tif.s and gif.s and computer stuff and Denise who does all the other stuff! And I miss Maureen, who always made work more than a little like play.

I have had the full support of my hospital colleagues, from the unbridled enthusiasm of Bernadette de Ruyter and Liam Dunbar, to the ongoing interest and support of Mr Nigel Willis and Mr Grant Kiddle in the orthopaedic department and Professor John Carter in haematology. Pacific Radiology were integral in completing the two haemodynamic studies, with the more than capable input from Stephen Mackintosh and Samantha Buchanan, and with access to radiology facilities at the most unusual hours!

Many of those who supported me have also become co-authors in some of the papers I have been fortunate enough to have published as a result of undertaking this thesis: Laird Cameron, Bridget Maher (née Healy), Matthew McKenzie, Judith Riley, Liam Dunbar Anthony Mallon, Bernadette de Ruyter, Samantha Buchanan, Stefan Ebmeier, Alex Semprini, Professor Weatherall and Professor Beasley. 
How would I have completed a PhD without study participants and patients? All 540 or so of them, who gave their time and a weensie bit of themselves so that we could all learn a bit more. Thank you.

Finally, the Health Research Council of New Zealand, who funded my studies with a Clinical Training Fellowship, and who support my workplace, the Medical Research Institute of New Zealand.

I am looking forward to catching up with some friends and family. I have missed them lately! They too have been very patient and I hope this makes them proud. 


\section{Table of Contents}

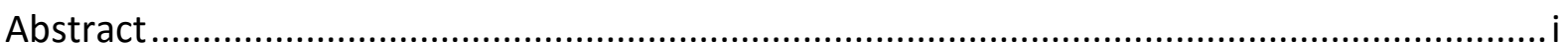

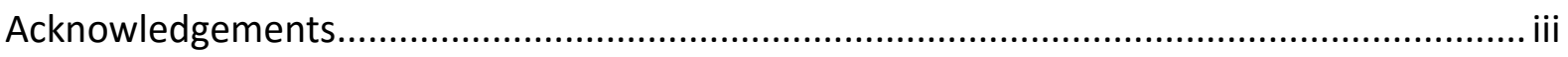

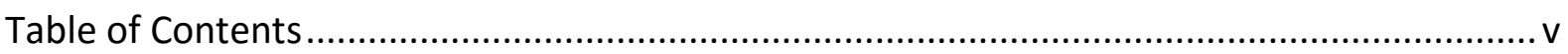

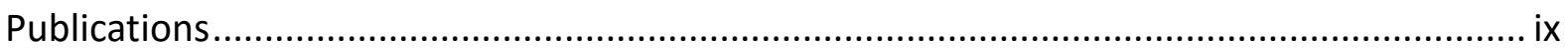

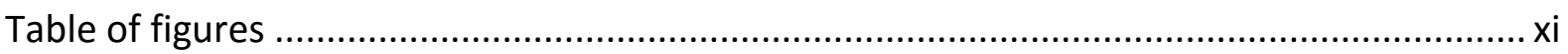

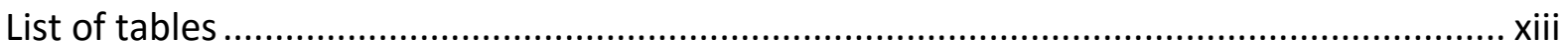

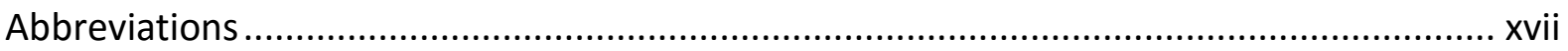

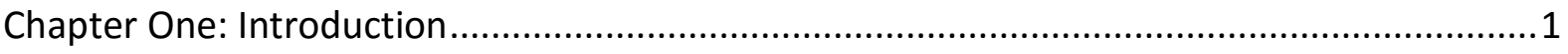

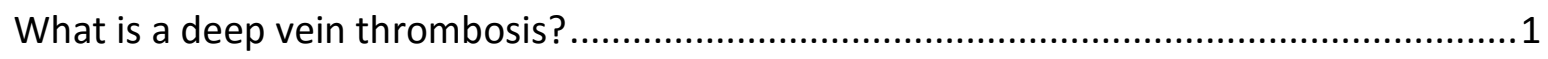

Prevalence of venous thromboembolism ....................................................................

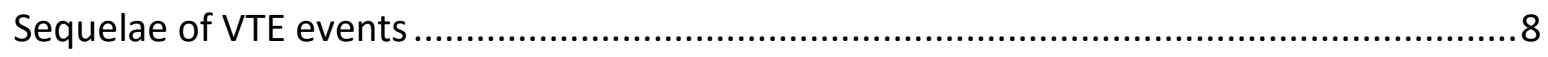

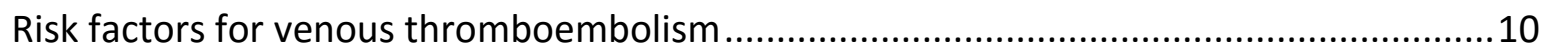

Hypercoagulability: the first component of Virchow's triad ........................................ 10

Endothelial wall injury: the second component of Virchow's triad ...............................13

Stasis: the third component of Virchow's triad .......................................................... 14

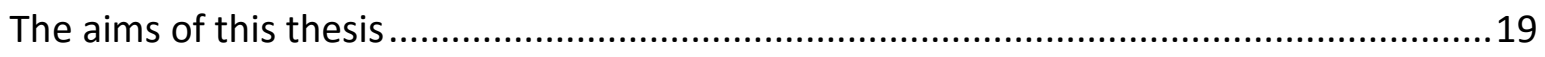

Chapter Two: Seated Immobility and venous thromboembolism ....................................23

The developing evidence for risk of venous thromboembolism with sedentary behaviours

Occupational sitting, computer use and venous thromboembolism 
STUDY ONE: Venous thromboembolism risk associated with protracted work- and computer-related seated immobility: A case-control study

STUDY TWO: Seated immobility and the risk of venous thromboembolism: a meta-analysis of two case-control studies.

Chapter Three: Lower limb immobilisation and the risk of venous thromboembolism

The medical literature: Prevalence of venous thromboembolism in lower limb trauma or immobilisation. .80

STUDY THREE: Lower limb immobilisation and venous thromboembolism risk: Combined Case-control studies .90

Chapter Four: Interventions for the prevention of venous thromboembolism in sedentary workers

STUDY FOUR: Legflow increases lower-limb venous haemodynamics when seated: a randomised controlled trial 108

Chapter Five: Interventions for the prevention of venous thromboembolism in the orthopaedic setting

Low molecular weight heparin

Rivaroxaban

Aspirin 136

STUDY FIVE: Venous thromboembolism rates in lower limb immobilisation after Achilles tendon injury unchanged after introduction of prophylactic aspirin: audit..... 140 Intermittent pneumatic compression therapy. .153

Intermittent pneumatic compression utilising foot pumps

STUDY SIX: Venous haemodynamics of Jet Impulse Technology within a lower limb fibreglass cast: a randomised controlled trial. (JIT in-cast trial) .165 
Chapter Six. Measurement of venous thromboembolism risk factors within the workforce, sedentary time at work and investigation of a possible intervention to promote venous blood flow of the lower limbs.

Validation of the Fitbit $^{\oplus}$ Flex $^{\mathrm{TM}}$ to measure the sedentary time during work regarding the 'Dome device at work study'

STUDY SEVEN: Measurement of venous thromboembolism risk factors within the workforce, sedentary time at work and investigation of a possible intervention to promote venous blood flow of the lower limbs: (Dome device at Work Study).....

Chapter Seven. A cohort feasibility study of an intermittent pneumatic compression device within a below-knee cast for the prevention of venous thromboembolism 209

STUDY EIGHT: A cohort feasibility study of an intermittent pneumatic compression device within a below-knee cast for the prevention of venous thromboembolism 213

Chapter 8: Conclusions 229

Appendix I. Venous thromboembolism risk associated with protracted work- and computer-related seated immobility: A case-control study. Participant Information Sheet

Appendix II. Venous thromboembolism risk associated with protracted work- and computer-related seated immobility: A case-control study. Questionnaire: Cases. .241

Appendix III: Venous thromboembolism risk associated with protracted work- and computer-related seated immobility: A case-control study. Questionnaire: Controls .....251 Appendix IV: Australian and New Zealand Classification of Occupations (ANZSCO): Level 2 Occupations .265

Appendix V: Participant information sheet: Legflow: Haemodynamic study..... 269 
Appendix VI: Participant Information Sheet: The effect of lower limb fibreglass cast on the efficacy of the Impulse IPC device on lower limb venous haemodynamics in healthy subjects.

Appendix VII: Validation of Fitbit ${ }^{\oplus}$ Flex $^{\mathrm{TM}}$ : Participant data

Appendix VIII: Participant Information Sheet: Sedentary occupations: measurement of venous thromboembolism risk factors within the workforce, sedentary time at work and investigation of a possible intervention to promote venous blood flow of the lower limbs. (Dome device at work study) .299

Appendix IX: Questionnaire: Sedentary occupations: measurement of venous thromboembolism risk factors within the workforce, sedentary time at work and investigation of a possible intervention to promote venous blood flow of the lower limbs. (Dome device at work study). .307

Appendix X: Participant Diary: Sedentary occupations: measurement of venous thromboembolism risk factors within the workforce, sedentary time at work and investigation of a possible intervention to promote venous blood flow of the lower limbs. (Dome device at work study).

Appendix XI: Participant Information Sheet: Venous thromboembolism prevention in lower leg injury requiring immobilisation: a feasibility study and open-label trial of Jet Impulse Technology (JIT) within-cast. (JIT in-cast study)

Appendix XII: Publisher permissions to reproduce journal articles.... 


\section{Publications}

To date the following manuscripts and abstracts of research presented or related to this thesis have been submitted or published.

1. Beasley R, Braithwaite I, Jurevics R. Gaming: a 21st Century variant of seated immobility thromboembolism. (submitted to Clinical Medical Journals)

2. Braithwaite I, Shirtcliffe P, Weatherall M, Beasley R. Anti-androgen contraceptive pills and higher risk of venous thromboembolism. NZMJ. 2016;1430:102-4

3. Braithwaite I, Healy B, Cameron L, Weatherall M, Beasley R. Venous thromboembolism risk associated with protracted work- and computer-related seated immobility: A case control study. JRSM open, 7(8), 2054270416632670. (STUDY ONE: Included in Chapter Two).

4. Braithwaite I, Weatherall M, Beasley R. Lower limb immobilisation and venous thromboembolism risk: combined case-control studies. Postgraduate Medical Journal, postgradmedj-2016. (STUDY THREE: Included in Chapter Two).

5. Braithwaite I, Mackintosh S, Mallon A, Weatherall M, Beasley R. Legflow increases lower-limb haemodynamics when seated: a randomised controlled trial. Occupational ergonomics. 2016; DOI 10.3233/OER-150231. (STUDY FOUR: Included in Chapter Four).

6. Braithwaite I, Dunbar L, Eathorne A, Weatherall M, Beasley R. Venous thromboembolism rates in lower limb immobilisation after Achilles tendon injury unchanged after introduction of prophylactic aspirin: audit. J Thromb Haemost. 2016;14:331-335. DOI: 10.1111/jth.13224. (STUDY FIVE: Included in Chapter Five).

7. Braithwaite I, Mackintosh S, Buchanan S, Mallon A, Schwarzenlander K, De Ruyter B, Weatherall $M$, Beasley R. Intermittent pneumatic compression device within a lower limb fibreglass cast and venous haemodynamics: a randomised controlled trial. (Accepted by JRSM Open; STUDY SIX: Included in Chapter Five).

8. Braithwaite I, de Ruyter B, Semprini A, Ebmeier S, Kiddle G, Willis N, Carter J, Weatherall $M$, Beasley R. A cohort feasibility study of an intermittent pneumatic compression device within a below-knee cast for the prevention of venous thromboembolism. BMJ open, 6(10), e012764. (STUDY EIGHT: Included in Chapter Seven). 


\section{Table of figures}

Figure 1.0: The coagulation cascade.

Figure 3.1: The proportion of cases with cast immobilisation in the four weeks prior to their index VTE event $(n=62)$ with other risk factors for VTE. Cases may have had more than one other risk factor.

Figure 4.1: The top panel shows the Legflow Device, and the bottom panel shows the position of the participant during the study.

Figure 4.2. Popliteal vein blood flow measurements in the immobile and Legflow mobilised legs at baseline, 15 and 20 minutes as assessed by duplex ultrasonography. Clockwise from top left panel: Peak Systolic Velocity, Mean Flow Velocity, Vein Diameter and Total Volume Flow.

Figure 4.3. Popliteal vein blood flow: change from baseline (Legflow mobilised limb minus immobile limb) at 15 and 20 minutes as assessed by duplex ultrasonography. The horizontal lines are the 25th, median, and 75th percentiles, symbols are the mean and the whiskers are from minimum to maximum. Clockwise from top left panel: Peak Systolic Velocity, Mean Flow Velocity, Vein Diameter and Total Volume Flow.

Figure 5.1. Vena Jet pump (VADOplex, Germany) and its' associated under-cast foot garment 162

Figure 5.2: Application process, IPC and cast 164

Figure 5.3. Flow of participants through study 169

Figure 5.4: Box-plot of peak systolic velocity in casted and non-casted leg at each time point. The horizontal lines are the $25^{\text {th }}$, median and $75^{\text {th }}$ percentile, the symbol is the mean and the whiskers are the minimum to maximum.

Figure 6.1: Fitbit $^{\circledR}$ Flex $^{\mathrm{TM}}$ as usually worn by a participant

Figure 6.2: Flow of participants through study 193 
Figure 6.3: Frequency of pre-existing risk factors for venous thromboembolism reported by participants (exclusive of prolonged work-related seated immobility).

Figure 6.4: Frequency of risk factors for venous thromboembolism reported by participants inclusive of prolonged work-related seated immobility...... 198

Figure 7.1: Placement of patient foot within JIT foot garment and fibreglass cast. 216

Figure 7.2: The VADOplex ${ }^{\circledR}$ VenaJet foot pump system. 217

Figure 7.3: Flow of eligible participants for study 219

Figure 7.4: Dorsal foot ulcer with IPC / JIT foot garment utilisation. .223 


\section{List of tables}

Table 2.1 Risk factors for venous thromboembolism

Table 2.2: Characteristics of study participants and univariate odds ratios between potential risk factors and venous thromboembolism. .46

Table 2.3: Univariate and multivariate associations between components of seated immobility and VTE event.

Table 2.4: Study participants by occupational group and sex, $\mathrm{N}=200$ for both cases and controls

Table 2.5: Risk factors for venous thromboembolism

Table 2.6: Characteristics of study participants.

Table 2.7: Study participants by occupational class.

Table 2.8: Odds Ratios $(95 \% \mathrm{Cl}) *$ for association of risk factors and VTE with prolonged workand computer-related seated immobility as a categorical variable.

Table 2.9: Odds Ratios $(95 \% \mathrm{CI}) *$ for association of risk factors and VTE with frequency of prolonged work- and computer-related seated immobility as a continuous variable.

Table 3.1: Retrospective reviews of data for VTE events in patient groups

Table 3.2: Placebo arms of randomised controlled trials involving lower limb immobilisation

Table 3.3: Prospective cohort studies involving lower limb immobilisation

Table 3.4: Characteristics of study participants

Table 3.5: Clinical situation for lower limb immobilisation in cases and controls .95

Table 3.6: Univariate Odds Ratios $(95 \% \mathrm{Cl})$ for association of risk factors and VTE. .96 
Table 3.7: The difference between the percentage of cases and controls with a potentially preventable risk factor for VTE.

Table 4.1: Popliteal vein blood flow measurements as assessed by duplex ultrasonography.

Table 4.2. Differences in popliteal vein blood flow between Legflow-mobilised limb and immobile limb at each time point.

Table 4.3: Comparison of popliteal venous haemodyamics measured in the Legflow study compared with other studies measuring the effects of mechanical and non-mechanical interventions in seated participants.

Table 5.1. Characteristics of patients with Achilles tendon injuries included in audit.

Table 5.2. Standard Capital and Coast District Health Board protocol for non-operative management of Achilles tendon injury by cast immobilisation

Table 5.3. Characteristics of patients who experienced venous thromboembolism (VTE) within 70 days of cast immobilisation for treatment of Achilles tendon injury.

Table 5.4: Popliteal vein blood flow measurements assessed by duplex ultrasonography..170 Table 5.5: Differences in popliteal vein blood flow between casted leg and non-casted leg at each time point.

Table 6.1: Comparison of Fitbit ${ }^{\circledR}$ Flex $^{\text {TM }}$ recorded time in five minute increment and documented active/ sedentary time by participants. 185

Table 6.2: Participant characteristics. 195

Table 6.3: Use of the Legflow reported in participant diary. 196

Table 6.4: Table of work-related sitting parameters reported by participants. 199

Table 6.5: Fitbit monitored work-related sitting and non-sitting times..... 201 
Table 6.6: Comparison of participant reported sitting time while at work and monitored sitting activity

Table 7.1. Flow of potential participants through the feasibility study

Table 7.2. Description of potential and enrolled participants in feasibility study .222 


\section{Abbreviations}

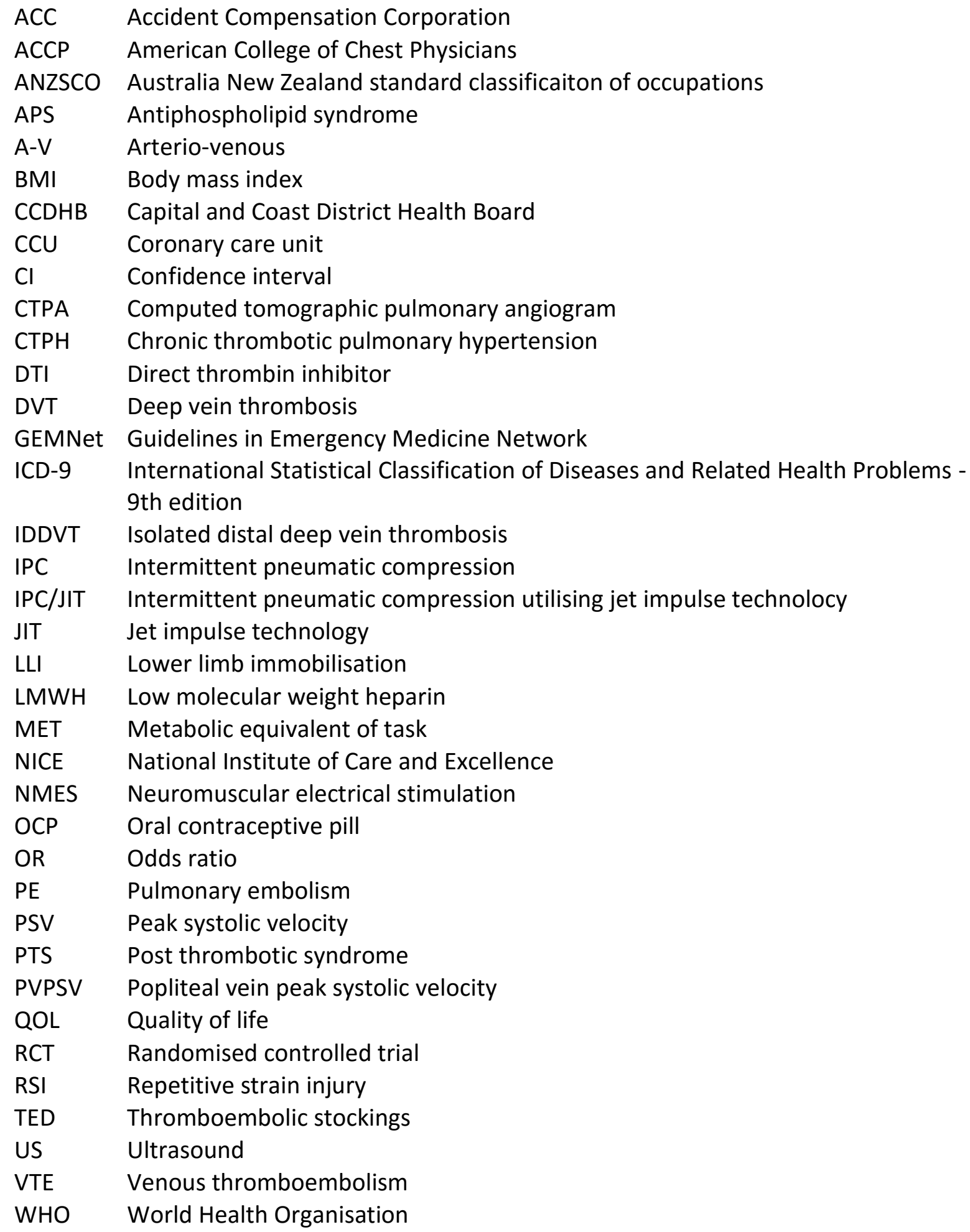




\section{Chapter One: Introduction}

\section{What is a deep vein thrombosis?}

A deep vein thrombosis (DVT) is a blood clot that forms in the deep vein of the leg or the arm. Veins are thin walled blood vessels that rely on a combination of internal valves and muscle contraction to return blood back to the heart. Blood flows from superficial veins close to the skin, via perforator veins into the deep veins of muscles. Veins have one way valves to ensure blood flows in only one direction when they are squeezed by muscles.

Haemostasis is a constant physiological response that prevents excessive blood loss after injury, and depends on a series of events involving platelets, endothelial cells, proteins of the coagulation cascade and the fibrinolytic pathway.

\section{The coagulation cascade}

The coagulation cascade is a series of biochemical events that result in the formation of a blood clot and its eventual dissolution (Figure 1.0). Factors that operate in this cascade are usually inert (in zymogen form) throughout the body until activated by a preceding insult or chemical when it undergoes biochemical change to release its active form. As shown in Figure 1.0 there are two initial pathways of coagulation that end in a common pathway. The intrinsic pathway is activated when zymogens are exposed to negatively charged surfaces that can be found on bacteria, large fat molecules and on the sub endothelial tissue, while the extrinsic pathway is activated by damage to tissue.

The 'cascade' is exactly that. In the case of the intrinsic pathway, exposure to a negative charge binds Factor XII to a sub-endothelial surface. A complex of prekallikrein and high molecular weight kininogen interacts with the same exposed surface and when in close enough proximity to the bound factor XII, activates that Factor, becoming Factor XIla. Factor XIla then activates Factor XI, which becomes Factor Xla, which activates Factor IX. Factor IXa binds with Factor VIII which in turn activates Factor X, the beginning of the common pathway. 
The extrinsic pathway is rapidly activated by tissue damage, and results in activation of Factor X via a single step pathway. Tissue factor (Factor III) is bound to the cell membranes of most human cells. When released Factor III binds to Factor VII, which is activated to VIIa. Factor VIla binds with calcium and phospholipids and rapidly activates Factor X.

The common pathway, the first common step of both the intrinsic and extrinsic pathways, starts at Factor X. Activated Factor X activates Factor II (Prothombin) which then forms Factor Ila (thrombin). Thrombin activates Factor I (Fribinogen) to Factor la (Fibrin) which will ultimately form a blood clot.

Not only does vascular endothelial injury initiate the clotting cascade, it also causes activation of platelets, which are bound to the endothelial collagen. This causes a calcium release attracts prothrombin to the platelet surface, resulting in a meshwork of activated Factor Ila (thrombin) surrounding the platelets, eventually forming an organised clot with the assistance of Factor XIIIa.

\section{Fibrinolytic pathway}

A concurrent antagonising cascade, the fibrinolytic pathway, is activated that ensures the clot is eventually dissolved, thus maintaining the balance between coagulation and anticoagulation (a homeostatic mechanism). When there is an imbalance between these two cascades a venous thrombosis may form. Venous thrombi in superficial and perforator veins are usually isolated events, as the one way valves tend to filter the blood clots and prevent them from entering the deep venous system. However, a blood clot forming the deep venous system can cause localised pain and swelling and parts of the clot may break off, becoming an embolus (pleural; emboli). Emboli can travel through the venous system, to the right side of the heart and into the pulmonary circulatory system, becoming lodged in the lung; a pulmonary embolism (PE). Collectively DVT and PE are known as venous thromboembolism (VTE). 
Figure 1.0: The coagulation cascade

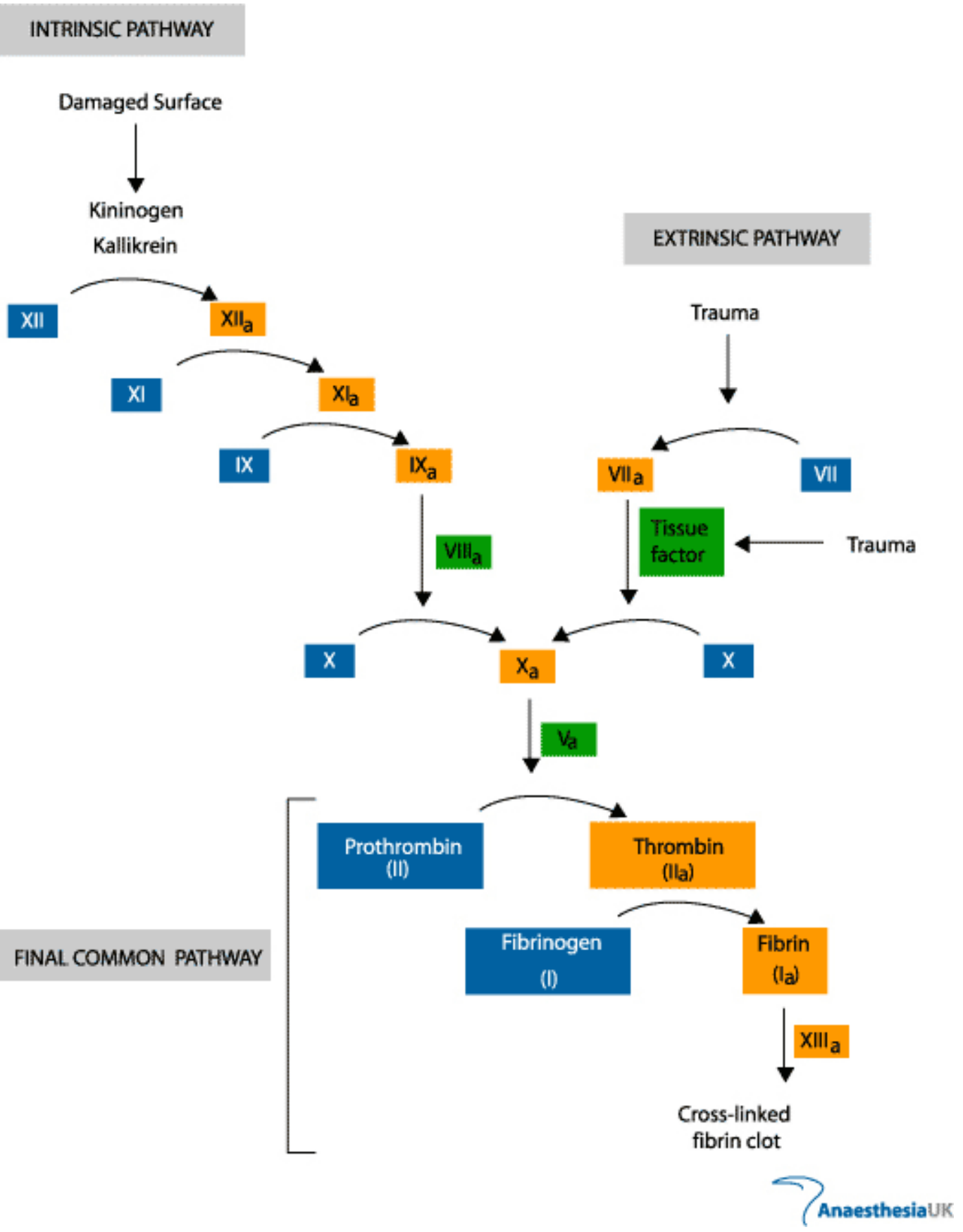




\section{Virchow's triad}

Thrombosis has been documented in the medical literature since as early as 2650 BC (Dickson, 2004), and venous thrombosis was first found in the literature in around 1400AD. Modern day understanding of risk factors for VTE appears to have started with Rudolf Virchow. While in Berlin in the mid-1800's, Virchow undertook as series of autopsies by which he determined that clots in the lungs appeared separated from a more distal source (DVTs in the legs), as the lung clots had a concave shape that matched the convex shape of the clots in the legs. He described the phenomenon of the lung clot dislodging from the more distal leg clot as 'embolism' (Virchow, 1856). Throughout the course of Virchow's writing the themes of 'alterations in the constitution of the blood' (hypercoagulability), 'vascular endothelial injury' and 'alterations in blood flow' (stasis), were developed in relation to the pathogenesis of VTE (Virchow, 1859). Over time, this combination of concepts in the pathogenesis of VTE has become known as 'Virchow's triad' and while the overall concepts of hypercoagulability, vascular endothelial injury and stasis have persisted, more specific risk factors for VTE have been identified and quantified. 


\section{Prevalence of venous thromboembolism}

In the absence of national VTE surveillance programmes, researchers across a range of countries and communities have attempted to estimate the incidence of VTE.

\section{United States}

In the United States (US) W Coon and colleagues reported thromboembolic events by age from within a Michigan community (the Tecumseh Community Health Study) in 1970. Extrapolated to 1970 US census figures, they estimated the annual incidence of DVT to be 22 and 27 per 10,000 person-years in men and women aged 46 years or greater respectively (Coon, Willis III, \& Keller, 1973). In a defined population in Massachusetts the average annual incidence of DVT and PE was found to be 4.8 per 10,000 and 2.3 per 10,000 personyears (Anderson Jr. et al., 1991). A study of the annual incidence of VTE in the population of Olmsted County Minnesota over a 25-year period (1966 to 1990) found an overall age and sex-adjusted incidence of VTE of 12 per 10,000 person-years (4.8 per 10,000 PEs and 6.9 per 10,000 DVTs) (Silverstein et al., 1998). The rate of VTE events increased with increasing age, but it was also noted that the rates of PE had reduced by almost half during the study period, while the rate of DVT had stayed the same for men, reduced for women under 55 years of age and increased for women over 60 years. Cushman and colleagues reported an age standardised rate of 19.2 per 10,000 person-years in two cohorts representing different regions of the US and noted that half of cases were idiopathic and that the short-term mortality and two-year recurrence rates were 'appreciable' (Cushman et al., 2004).

\section{Europe}

Similar rates of VTE have been reported in Europe. In 1992 Nordström and colleagues reported an incidence of 16 events per 10,000 inhabitants per year in the city of Malmö, Sweden, and described an increasing risk with increasing age (Nordström, Lindblad, Bergqvist, \& Kjellström, 1992). In Brest, France, the annual incidence of VTE was found to be 18.3 per 10,000 person-years, with an incidence of DVT and PE of 12.4 and 6.0 per 10,000 person-years respectively (Oger, 2000). In 2007 Cohen and colleagues estimated that across six European Union countries (France, Germany, Spain, Italy, Sweden and the United 
Kingdom) there were over one million VTE events of which 466,000 were DVT, 296,000 were PEs and 370,000 were VTE-related deaths (AT Cohen et al., 2007).

\section{Australia and New Zealand}

In Australia the annual incidence of VTE has been estimated at 10 per 10,000 person-years, resulting in approximately 20,000 episodes of VTE per annum (National Institute of Clinical Studies, 2005), and by extrapolation, the New Zealand VTE Prevention Programme group estimated that there would be approximately 4,000 VTE events per annum in New Zealand (New Zealand Venous Thromboembolism Prevention Steering Group, 2012).

\section{Background risk in the general population}

In a systematic review of studies on the incidence of VTE in the general population which included nine studies mainly from the US and Sweden, Fowkes and colleagues estimated that in the general population between five and 10 per 10,000 people per annum would be diagnosed with a first incidence of DVT, of which approximately $40 \%$ would have no clear cause (Fowkes, Price, \& Fowkes, 2003).

\section{Fatality rates associated with VTE}

In around $25 \%$ of PE cases, the initial presentation is sudden death (J. Heit, 2008). Ageadjusted PE fatality rates in the US are estimated to be 0.9 per 10,000 person-years (Gillum, 1987). In the United Kingdom, it is estimated that 25,000 deaths annually are VTE related (House of Commons Health Committee, 2005). In Australia, there are an estimated 2,000 VTE-related deaths annually (National Institute of Clinical Studies, 2005). In-hospital casefatality rates from VTE are approximately $12 \%$, and at 1,2 , and 3 years can reach $19 \%, 25 \%$ and 30\% respectively (Anderson Jr. et al., 1991). This is corroborated by Prandoni and colleagues who estimated a survival rate after VTE at 8 years is approximately $70 \%$ (Prandoni et al., 1996). Between 10 and 30\% of all VTE patients die within 30 days of presentation (Beckman, Hooper, Critchley, \& Ortel, 2010; Cushman et al., 2004).

\section{Limitations of estimating background VTE rates}

In nearly all studies, the authors have indicated that the rates are estimations based on detected events, and that many VTE events may be undetected as autopsy rates have 
declined over the last few decades (J. A. Heit, 2005; Oger, 2000; Silverstein et al., 1998). Notwithstanding the possible underestimation of VTE events, VTE has been cited as a common cause of cardiovascular death, third in frequency behind acute coronary syndrome and cerebrovascular accident (Goldhaber \& Bounameaux, 2012). 


\section{Sequelae of VTE events}

Complications of VTE include a reduced quality of life (QOL), post-thrombotic syndrome (PTS) with or without venous ulceration, chronic thromboembolic pulmonary hypertension (CTPH) and VTE recurrence (Stein \& Matta, 2010).

\section{Quality of Life}

Survivors of VTE events experience a poorer QOL than population norms, and about one third of patients suffer a reduction in QOL within four months of their VTE event (Kahn et al., 2005).

\section{Post thrombotic syndrome}

PTS signs and symptoms include chronic leg pain, swelling, skin discolouration, varicose veins and ulcers. This condition is associated with a reduced quality of life (Kahn et al., 2005). Prandoni and colleagues estimate that one quarter of VTE patients develop PTS within two years, and nearly $30 \%$ within eight years (Prandoni et al., 1996). The risk of PTS is substantially increased with proximal DVTs. The ten-year cumulative risk in this clinical context is between $60 \%$ and $100 \%$ (Ashrani \& Heit, 2009).

\section{Chronic thromboembolic pulmonary hypertension}

CTPH is a disease of the pulmonary vasculature caused by chronic obstruction of the pulmonary arteries, resulting in a mean pulmonary arterial pressure of greater than $25 \mathrm{mmHg}$. It presents with increasing exertional dyspnoea, and occasionally syncope and is a long-term complication of PE in up to $10 \%$ of symptomatic patients. CTPH is also surmised to be a long term consequence of asymptomatic PEs (Lang \& Madani, 2014). CTPH can be cured by pulmonary arterectomy, however, due to a long asymptomatic period and gradual disease progression, the condition may often be well progressed by diagnosis, reducing the chance of operative cure. Estimated survival of operated patients is $89 \%$ at 3 years, while non-operated patients have a three-year survival of $70 \%$ (Delcroix et al., 2016). Death is usually caused by right ventricular failure due to a progressive increase in pulmonary arterial pressure. 


\section{Recurrence of venous thromboembolism}

Five to $14 \%$ of patients with VTE are readmitted with a recurrence within one year of their initial diagnosis (Cushman et al., 2004; Spyropoulos \& Lin, 2007) with 50 and 59\% of the DVT and PE readmissions occurring within 90 days respectively. Approximately one third of VTE patients experience a recurrence of VTE within 10 years of completing their initial course of treatment (J. Heit, 2008).

\section{The costs of venous thromboembolism}

In the US in 2007, the cost associated with the treatment of a newly diagnosed DVT or PE was estimated at $\$ 10,804$ and $\$ 16,644$ respectively (Spyropoulos \& Lin, 2007). Since then Beckman and colleagues have extrapolated that the annual costs associated with new VTE are between two and 10 billion US dollars (Beckman et al., 2010). With significant comorbidities arising from VTE events and the increased risk of recurrence as well as the costs associated with treatment of VTE and its sequelae, it is clear that VTE imposes a significant burden on health systems worldwide. 


\section{Risk factors for venous thromboembolism}

There are an increasingly large number of identified risk factors which, either alone, or when interacting with environmental, behavioural or genetic factors, or with each other, are associated with VTE. These risk factors, along with treatment modalities, can generally be classified into one of the components of Virchows' triad.

\section{Hypercoagulability: the first component of Virchow's triad}

In this component of Virchows triad, risk factors can be genetic or acquired, and include hereditary thrombophilias, antiphospholipid syndrome, increasing age, overweight and obesity, cancers and their treatments, pregnancy and the post-partum period, exposure to hormonal therapies such as oral contraceptive pills and hormone replacement therapy, smoking and acute and chronic medical conditions.

\section{Hereditary thrombophilia}

In 1965 Egeberg wrote the first report of an autosomal dominant genetic mutation causing a deficiency of antithrombin that presented in a Swedish family as a hereditary tendency to VTE, described as thrombophilia (Egeberg, 1965). Since then, protein C and protein S deficiencies have been described. Combined, deficiencies of antithrombin, protein $\mathrm{C}$ and protein S account for less than 10\% of VTEs (Kujovich, 2011). Resistance to protein C inferred by a Factor $V$ Leiden mutation is the most common genetic thrombophilia, and can be found in approximately $50 \%$ of families with thrombophilic tendencies and approximately $20 \%$ of individuals presenting with VTE (Kujovich, 2011). The mutation can be in one gene (heterozygous) or in both genes (homozygous).Two further genetic causes of thrombophilia have been identified; increased concentrations of Factor VIII associated with blood group $\mathrm{O}$, and a mutation in the prothrombin (Factor II) gene found in approximately $6 \%$ and $20 \%$ of patients presenting with VTE respectively (Rosendaal, 2005). 


\section{Antiphospholipid syndrome}

Antiphospholipid syndrome (APS) is a systemic automimmune condition, conferring increased risk of thrombosis (arterial and venous) and miscarriage. It is characterised by the presence of antiphospholipid antibodies (anticardiolipin antibodies, Lupus anticoagulant). It can occur independently (Primary APS) or in conjunction with other autoimmune disorders.

\section{Age}

A number of studies have found an association between increasing age and risk of VTE (Anderson Jr. et al., 1991; Coon et al., 1973; Silverstein et al., 1998; Stein \& Matta, 2010). Patients 70 years or older are six times more likely to be diagnosed with PE and 13 times more likely to be diagnosed with DVT than patients aged 20 to 29 years (Stein \& Matta, 2010).

\section{Cancer}

Cancers and their treatments are associated with a seven-fold higher risk of VTE. VTE is the second leading cause of death in cancer patients (Blom, Doggen, Osanto, \& Rosendaal, 2005). The risk depends on the stage of the cancer, the tumour type and location and treatment modalities employed (A. Lee \& Levine, 2003). Metastatic lung disease and concomitant chemotherapy are associated with higher VTE incidence (Walker et al., 2016). Breast cancer treatments, including Tamoxifen (an oestrogen receptor modulator) and the placement of central venous catheters during treatment are associated with increased risk of VTE (Rebouças et al., 2016). Doxorubicin and prolonged treatment with chemotherapy for Non-Hodgkins lymphoma are associated with higher risks of VTE (Sanfilippo et al., 2016). Because cancers are often associated with other risk factors for VTE, it can be difficult to establish the exact increased risk due to the cancer itself. However, there is evidence that some cancers express proteins that can upregulate prothrombotic factors in the human coagulation cascade (Nickel et al., 2016) which would indicate that cancer alone may cause VTE. 


\section{Pregnancy}

Pregnancy has been associated with VTE, with approximately one diagnosed VTE per 1,600 births. Most DVTs are ante-partum, but most PEs seem to occur postpartum and are associated with caesarean section (Gherman et al., 1999).

\section{Oestrogen exposure}

With the increasing availability of oral oestrogen compounds for contraception in the 1960s, there was an increased rate of VTE in young women to whom they were prescribed. As oestrogen doses in oral contraceptive pills (OCPs) have decreased over time, there remains a smaller but still increased risk of VTE. Compared to non-users of OCPs, those using OCPS containing desogestrol, gestodene and drospirenone have a six to seven-fold increased risk of VTE. Those using OCPs containing levonorgestrel have a three-fold increased risk of VTE. The risk of VTE is not increased for those using the progestogen only pill (Lidegaard, Nielsen, Skovlund, Skjeldestad, \& Lokkegaard, 2011). For those taking hormone replacement therapy after menopause the risk of PE is increased two-fold (Grodstein et al., 1996).

\section{Obesity}

Obesity (body mass index (BMI) greater than $30 \mathrm{~kg} / \mathrm{m}^{2}$ ) has been shown to induce changes in the coagulation cascade (Darvall, Sam, Silverman, Bradbury, \& Adam, 2007). Doppler investigations of limbs in obese individuals has shown greater vein diameter, lower venous amplitude and lower shear stress in the femoral vein compared to non-obese controls potentially leading to increased risk for VTE (Willenberg et al., 2010). Despite this the epidemiological evidence for obesity as an independent risk factor for VTE is unclear.

In the Longitudinal Investigation of Thromboembolism Etiology (LITE) study, information from two prospective cohort studies of the risk factors of arterial disease were combined and the same risk factors were assessed for association with VTE (Tsai et al., 2002). The hazard ratio for VTE with a BMI between 35 to $40 \mathrm{~kg} / \mathrm{m}^{2}$ was 2.5 , and for BMI greater than $40 \mathrm{~kg} / \mathrm{m}^{2}$ was 2.7 . In a prospective cohort study in the UK, overweight was significantly associated with risk of VTE (Huerta, Johansson, Wallander, \& García, 2007). 
However, as early as 1978 in a follow up study of 564 morbidly obese patients who underwent gastric bypass surgery, only seven developed PE and of 57 who had Doppler examinations of the lower limb, only one had a DVT (Printen, Miller, Mason, \& Barnes, 1978). More recently Heit and colleagues have found no association between BMI and VTE in their analysis of data from the Olmsted, Minnesota study (J. Heit et al., 2000). The conflicting findings suggest that obesity and overweight may interact with other risk factors to precipitate VTE without necessarily being independent risk factors themselves.

\section{Atherosclerotic disease}

The clinical evidence for an association between VTE and atherosclerotic disease, cardiovascular disease and cerebrovascular disease in particular, is mixed. In population studies, cardiovascular disease and heart failure have been associated with increased risk of VTE (Huerta et al., 2007). In studies of cohorts with diagnosed risk factors for cardiovascular and cerebrovascular disease, an increased risk of VTE with these same risk factors has not been demonstrated, or has been limited to an association with only a few risk factors such as obesity and diabetes (Glynn \& Rosner, 2005; Tsai et al., 2002).

\section{Other}

Nephrotic syndrome (Llach, 1985), major trauma (Geerts, Code, Jay, Chen, \& Szalai, 1994), burns (Sevitt \& Gallagher, 1961) and cigarette smoking (Hansson, Eriksson, Welin, Svardsudd, \& Wilhelmsen, 1999) have also been associated with increased risk of VTE due to their likely effect hypercoagulative effect.

\section{Endothelial wall injury: the second component of Virchow's triad}

Endothelial wall injury generally relates to piercing of a major blood vessel with a foreign body such as a central venous catheter and catheter-associated sepsis (Timsit et al., 1998), and / or damage from internal shear stress or hypertension (AT Cohen et al., 2007). However, chronic inflammation causing activation of membranes of monocytes (Grainge, West, \& Card, 2010) and sepsis with exposure to pro-coagulant surfaces of bacteria and platelet activation (Dellinger et al., 2004), causing activation of tissue factor and activation of the coagulation cascade are also in this category (Manly, Boles, \& Mackman, 2011). 
While hypercoagulability and endothelial wall injury, are two major components of Virchow's triad and are important considerations in the assessment, treatment and decisions with respect to the need for ongoing prophylaxis of VTE, it is not the focus of this thesis. Instead, this thesis will focus on particular aspects of stasis, the third component described in Virchow's triad.

\section{Stasis: the third component of Virchow's triad}

Risks associated with stasis include varicose veins, venous stasis, long surgical operations, and prolonged immobility (as on a long plane or car ride, or bed bound during hospitalization). In many of these instances, there is also endothelial wall injury and activation of the coagulation cascade, leading to hypercoagulability.

Immobility is associated with venous stasis, most often around venous valves, causing inflammation and hypercoagulability (Bovill \& van der Vliet, 2011; Virchow, 1856). The underlying premise is that severe hypoxia occurs in the deepest recesses of venous valvular structures as a result of prolonged stasis This hypoxia induces an adaptive response in the endothelial cells, upregulating genes associated with coagulation, inflammation and angiogenesis (Bovill \& van der Vliet, 2011), thereby increasing the risk of VTE.

\section{Varicose veins}

Varicose veins are enlarged, tortuous blood veins caused by stretched and inelastic vessel walls and / or damaged or weakened valves allowing the backflow of blood into the vein, resulting in engorged, dilated blood vessels. They may occur anywhere in the body, but are most commonly present in the legs and feet, most likely due to the gravitational demands of standing upright and walking. Varicose veins are present in approximately three quarters of adult females and over half of adult males (Beebe-Dimmer et al., 2005). Stasis associated with pooling in the varicose vein can lead to hypoxic injury and initiation of the coagulation cascade. Varicose veins are an age-dependent risk factor for VTE with a diminishing risk for age (J. Heit et al., 2000). Risk factors for varicose veins include increasing age, sex, pregnancy, obesity, standing for prolonged periods of time and family history of varicose veins. 


\section{Inpatient surgery}

Long surgical operations, hospitalisations and consequent immobility are closely related. Previous studies have shown that the risks of VTE in the clinical setting of surgery are high. In the Million Women Study, Sweetland and colleagues found that women were 70 times more likely to be admitted with VTE in six weeks of an inpatient operation, and ten times more likely after a day-case operation compared to women who had not had surgery (Sweetland et al., 2009). The VTE risk (although reduced compared to the first six weeks post-operatively) persisted between seven and 12 weeks; (ten times more likely after inpatient surgery and 5.5 times more likely after day-case surgery). They also found that the risk varied considerably by type of operation, with the highest relative risks for hip and knee surgery (220-fold increased risk) and for cancer (92-fold increase in risk).

Qu and colleagues have reported that overall incidence of VTE in women undergoing gynaecological surgery was $9.2 \%$ and that independent variables associated with increased risk of VTE in this setting were varicose veins (OR 4.64), bed rest greater than 48 hours (OR 3.94), operation length greater than three hours (OR 3.31), laparotomy surgery (OR 2.51), hypertension (OR 2.13) and age greater than 50 years (OR 2.10) (Qu et al., 2015).

In orthopaedic surgery, high risk operations for symptomatic VTE have also been identified despite prophylaxis; internal fixation of pelvic fractures (12\%), knee replacement surgery (3.7\%), internal fixation of proximal tibial fractures (3.8\%), and internal fixation of ankle fractures (3.6\%) (L. Lapidus, Ponzer, Pettersson, \& de Bri, 2013). Additionally high rates have been found after surgical achilles tendon repair (7.2\%), an orthopaedic operation that does not routinely receive prophylaxis (L. Lapidus et al., 2013).

Other high risk surgeries have also been identified, and VTE risk may be variable depending on interactions with patient age and the presence or absence of cancer (White, Zhou, \& Romano, 2003). White and colleagues analysed records from 76 different categories of surgery (1.65 million patients) and reported a number of surgeries with VTE rates greater than $2 \%$, which they recommended should be categorised as high risk surgeries; invasive neurosurgery (2.3\%), major vascular surgery (2.8\%) and major hip surgery $(2.4 \%)$. There are now international guidelines for the prevention of VTE in many of these inpatient surgical 
settings (Dellinger et al., 2004; Falck-Ytter et al., 2012; National Institute for Health and Clinical Excellence, 2010; NHS, 2010). However White also identified that $56 \%$ of VTE events occurred after hospital discharge and recommended extended duration prophylaxis after high risk surgery.

\section{Post-discharge venous thromboembolism}

Spencer and colleagues analysed hospital records of residents from Worcester Massachusetts between 1999, 2001 and 2003 and found that 74\% of VTE patients had developed their VTE event in the community. Of these, $23 \%$ had undergone surgery in the preceding three months and 39\% had been hospitalised. In 2014 Engbers and colleagues reported findings from the Age and Thrombosis - Acquired and Genetic risk factors in the Elderly (AT-AGE) study, a case-control study of 401 adults aged greater than 70 years with a first time VTE (cases) and 431 adults who had never had a VTE (Engbers, Blom, Cushman, Rosendaal, \& Van Hylckama Vlieg, 2014). They found that there was a 15-fold increased risk of VTE within two weeks of discharge from hospital and that over the three month period post-discharge, fractures conferred a 13-fold risk of VTE, followed by surgery, plaster cast, transient immobility at home and minor leg injuries (6.6-, 6.2-, 5.0- and 1.9-fold increased risk of VTE respectively). When considering this patient group, it is difficult to make a distinction between the effect of hospitalization, surgery, a plaster cast, associated transient or extended immobility and the effect of the injury or elective operation itself.

\section{Immobilisation at home and venous thromboembolism}

Immobilisation in the community as opposed to within a hospital also confers an increased risk of VTE. Nauffal and colleagues have reported a retrospective study from a multicentre, international registry of acute VTE events in Italy (RIETE database) where they compared the three month mortality rate in patients with PE with patients categorised to the presence of recent immobilisation (greater than or equal to four days), recent surgery or neither of the two (Nauffal et al., 2012). In the patients who presented with PE, $23 \%$ had suffered recent immobilisation and $12 \%$ had recent surgery. Half of the patients who died from PE had been recently immobilised (43\%) or had surgery (7\%). Importantly, patients with immobilisation 
were 2.2 times more likely to suffer a fatal PE, and there was no difference in risk between whether immobilisation was community or hospital based.

In a follow up study of non-hospitalised patients from the same RIETE database, LopezReyes reported that twice as many patients developed acute VTE at home than in the hospital. Therefore, because the fatality rate was the same between the two groups, twice as many patients died from PE after being immobilised at home than in the hospital (LopezReyes et al., 2015). As well as immobilisation due to illness at home, paresis due to neurological conditions confers a three-fold risk of VTE (Clements, Churilov, Wahab, \& Ng, 2016; Coon, 1976; J. Heit et al., 2000), and high rates of VTE have been found in rest home residents (J. Heit et al., 2002).

\section{Seated immobility}

Aside from hospitalisation or surgery, other clinical settings involving prolonged seated immobility such as long distance travel (S. C. Cannegieter, Doggen, van Houwelingen, \& Rosendaal, 2006; Ferrari \& Morgan, 2004), working in cramped conditions (Holtermann et al., 2010) and even attending the theatre (Homans, 1954) have been associated with increased risk of VTE. While travel-related thrombosis is now a well-recognised risk factor for VTE, seated immobility both at work and for computer use is a setting in which there is emerging evidence of increased risk of VTE, and until recently has been described mainly in case reports and case-series in the medical literature (See Chapter Two).

\section{Isolated lower limb immobilisation}

Isolated lower limb immobilisation is also a known risk factor for VTE (Engbers et al., 2014; J. Heit et al., 2000; Huerta et al., 2007). However, lower limb immobilisation occurs in a variety of clinical settings including acute or elective surgery, traumatic injury, inpatient management with associated hospital immobility, and outpatient management. Lower limb immobilisation can be utilised in the management of soft tissue injuries and fractures of foot or leg bones. Additionally, lower limb immobilisation can comprise a range of modalities including plaster casts, fibreglass casts, moonboots and external fixation, and can include a range of weight-bearing options throughout immobilisation including; non weightbearing, partial weight-bearing, touch weight-bearing or full weight-bearing. 
Not only this, but the clinical significance of the increased risk of VTE in the setting of belowknee lower limb immobilisation is contested due to debate about the true incidence of clinically important and / or symptomatic VTE events and the risks of applying the usual practice of VTE prophylaxis such as an anticoagulant to this large clinical population.

Although there is an evidence base of the efficacy of low molecular weight heparin (LMWH) in reducing the risk of VTE in the setting of lower limb immobilisation (Mark Testroote et al., 2014), this has not been reflected in evidence-based guidelines for VTE prophylaxis. Rather the recommendation has been made to undertake further research in this setting (FalckYtter et al., 2012; National Institute for Health and Clinical Excellence, 2010; Randelli et al., 2013)

\section{Two elements of stasis under investigation for this thesis}

The two clinical settings of prolonged work- and computer-related seated immobility and prolonged lower limb immobilisation, each involving an element of stasis are the focus of this thesis. 


\section{The aims of this thesis}

There were two main aims to this thesis. The first comprised investigation of the association between prolonged work- and computer-related seated immobility and an extension of this area of research into an investigation of a possible preventive intervention, the Legflow device; a small innovative device for sedentary office workers to use while sitting. Secondly in a similar manner to seated immobility, I investigated the risk of VTE associated with lower limb immobilisation and extended this area of current clinical knowledge by assessing the practicality and feasibility of a non-pharmacological intervention, an intermittent pneumatic compression (IPC) device for the prevention of VTE within a below-knee fibreglass cast.

To investigate the association of prolonged work- and computer-related seated immobility and below knee lower limb immobilisation and the risk of VTE, I undertook the following activities:

- A literature review of VTE risk associated with seated immobility including air travel, other modes of travel and occupations.

- Study One: a case-control study of 200 cases and 200 sex and age-matched controls to determine whether prolonged work- and computer-related seated immobility within the sedentary work environment is a specific risk factor for VTE and to assess whether prolonged work- and computer-related seated immobility has been considered by treating clinicians as potential etiological factors in the development of VTE.

- Study Two: combined the data from the study above, with data from a similar study undertaken by Healy et al (B. Healy, Levin, Perrin, Weatherall, \& Beasley, 2010) giving a total of 396 cases and 397 controls with the objective of determining whether specific Australia New Zealand Standard Classification of Occupation (ANZSCO) classes and sub-classes are more at risk for VTE than others, e.g. Information, Communication and Technology (ICT) professionals. 
- A literature review of VTE risk, current prophylactic options and prophylactic guidelines in the setting of major orthopaedic surgery and in temporary lower limb immobilisation.

- Study Three: analysed the same combined case-control data from the 397 cases and 397 controls to determine the risk for association of lower limb immobilisation with VTE.

- Study Four: an audit of the current rates of VTE associated with Achilles tendon injuries treated with temporary immobilisation of the lower limb at Wellington Regional Hospital, Capital and Coast District Health Board (CCDHB), Wellington, New Zealand after the introduction of once daily aspirin (100mg) for VTE prophylaxis in this clinical setting.

I then assessed the haemodynamic effects of two devices; the Legflow (Medical Research Institute of New Zealand, Wellington, New Zealand), a novel floor-based foot-device, in the setting of prolonged work- and computer-related seated immobility, and an intermittent pneumatic compression (IPC) device for the foot which uses Jet Impulse Technology (IPC/JIT) in the setting of lower limb immobilisation as follows;

- Study Five: a comparison of the lower limb haemodynamics resulting from the use of the Legflow device in ten adult participants by comparing peak systolic velocity (PSV) in the popliteal vein of the limb using the Legflow with the PSV in the popliteal vein of the non-mobilised limb.

- Study Six: a comparison of the lower limb popliteal vein haemodynamics resulting from the application of an intermittent pneumatic compression (IPC/JIT) device within and without a below-knee fibreglass cast in 24 adult participants. I compared IPC/JIT haemodynamic performance inside the cast to unencumbered IPC by measuring the PSV in the popliteal vein and assessed ease of application of a cast over an IPC/JIT device, participant comfort and tolerability of the IPC/JIT device within a lower limb fibreglass cast. 
And finally I undertook feasibility studies of both devices to assess the practicality and utilisation of the each of them in their intended patient group and patient environment, comprising;

- Study Seven: a feasibility study in a convenience sample of desk-based workers to determine the feasibility of utilising the Legflow in the workplace environment by measuring adherence with recommended usage over a one week period, to characterise the known risks for VTE in this workforce sample, and to review participant-reported sitting behaviours with respect to VTE risk.

- Study Eight: a feasibility study of a minimum of 4-weeks treatment with either an IPC/JIT device plus aspirin $100 \mathrm{mg}$ daily within the cast of 70 patients following stable ankle fracture or post-foot/ankle surgery in order to assess the proportion of eligible subjects likely to enrol in a large randomised controlled trial that would assess the efficacy of the IPC/JIT and aspirin, versus aspirin alone, versus Rivaroxaban 10mg daily in the reduction of VTE in patients undergoing lower limb immobilisation.

My findings are presented in the following thesis and are submitted to the Victoria University of Wellington in fulfilment of the requirements for the degree of Doctor of Philosophy. 


\section{Chapter Two: Seated Immobility and venous thromboembolism}

In the last 50 years there have been significant changes in the relative proportions of time spent undertaking physical activity and sedentary time, particularly in developed countries. In the US The 'urban sprawl' between 1950 and the year 2000 led to just over half of households being situated within one mile of their local school or shopping centre, personal vehicle ownership increasing to an average of 1.9 vehicles per household, and by the year 2000 over $88 \%$ of commuting to and from work being undertaken in private vehicles (Brownson, Boehmer, \& Luke, 2005).

By the year 2000 only $25 \%$ of adult Americans were achieving the recommended levels of physical activity (30 minutes of moderate intensity activity on at least five days of the week). Two thirds of youth were achieving the recommended level of activity in 2001 (20 minutes of activity that made them breathe hard three times per week), but were less likely to be achieving this at school (32\%) compared to ten years previously (41\%).

In 1950 , only $10 \%$ of households had a television. By the year $2000,98 \%$ of households had at least one television. Average television viewing has increased by 36 minutes per decade since 1950, with average daily viewing times approaching eight hours per day by 2000.

In 2011, 76\% of US households reported having a computer, compared to $62 \%$ in 2003 and $8 \%$ in 1984 (File 2013). Internet access increased to $72 \%$ of households in 2011, compared to $55 \%$ in 2003 and $18 \%$ in 1997.

The proportion of the labour force in high activity occupations has fallen from approximately $30 \%$ in 1970 to $23 \%$ in 2000 , while the proportion of people in low activity occupations remains fairly stable at approximately 41\% (Brownson et al., 2005).

\section{Sedentary behaviour and associated health risks}

In 2013, concerns about physical inactivity and its contribution to worldwide mortality and morbidity resulted in the member states of the World Health Organisation (WHO) agreeing to a target of reducing insufficient physical activity by $10 \%$ by 2020 , as it is now regarded as 
a key modifiable risk factor in the prevention of non-communicable diseases (World Health Organisation (WHO) 2013).

In a 14-year follow up study of 113,000 American adults, sitting for greater than 6 hours a day was associated with a 30\% greater risk of mortality compared to sitting for less than 3 hours a day (Patel et al. 2010). Time spent sitting was independently associated with total mortality, regardless of activity levels. In a study of the US National Health and Nutrition Examination Survey (NHANES) participants aged over 50 years who wore an accelerometer, participants in the third quartile of sedentary time had a four-fold increased risk of death compared to the first quartile, and those in the fourth quartile of sedentary time had a sixfold increased risk of death (Koster et al. 2012).

In an analysis of data from a pre-existing study (the National Institute of Health - American Association of Retired Persons study), nearly 241,000 American adults aged 50 to 71 years with no cancer, cardiovascular disease or respiratory illness at baseline were followed for 8.5 years. Sedentary behaviours were positively associated with all-cause mortality. Participants with the most television viewing (equal to or greater than seven hours) had a $60 \%$ greater likelihood of all-cause mortality, $85 \%$ more likely to die from a cardiovascular disorder and $22 \%$ more likely to die from cancer compared to those who watched television for one hour or less per day. This increased risk persisted, even when adjusted for moderate to vigorous physical activity (Matthews et al., 2012).

In a prospective study of over 17,000 Canadians followed for an average of 12 years, increased sitting time was associated with a higher risk of mortality from all causes, particularly cardiovascular disease, with a dose response association between increasing sitting time and mortality from cardiovascular disease and all-cause mortality (Katzmarzyk et al. 2009).

In a population-based study of 45 to 75 year old people living in the United Kingdom, selfreported television viewing was associated with markers of obesity (Jakes et al., 2003). Each one hour increment in television watching was associated with an $11 \%$ increased risk of allcause mortality, with an apparent dose response effect (Dunstan et al. 2010). 
While many of these studies have focused on cardiovascular disease and cancer risk, they have also reported on all-cause mortality, and VTE mortality must also be included in the mortality statistics. Even in these large population studies, the frequency of VTE as a fatal event, the lack of autopsies at death and variable death certificate documentation would make it very difficult to quantify the proportion of fatalities attributable to VTE.

\section{Occupational sedentary behaviour and associated health risks}

Health risks associated with occupational sedentary time is an emerging area of research. In 2005, Mummery and colleagues reported that in a sample of 2,500 working Australian adults, occupational sitting time was independently associated with overweight and obesity in men who were in full time work (Mummery et al. 2005). In 2008, Healy and colleagues reported an association between breaks in sedentary time at work and a reduction in biological markers of metabolic risk, recommending that sedentary times were actively broken up in the working environment as a result (G. N. Healy et al., 2008). The Danish Work Environment study has reported that there is an association between increased occupation sitting time and BMI in women, but not in men (Eriksen, Rosthøj, Burr, \& Holtermann, 2015), and have also reported that despite in increased occupational sitting times over the last decade, this has not been offset by increased physical activity during leisure time (Jans, Proper, \& Hildebrandt, 2007).

While there has been a focus on reducing sedentary occupational time as a result of growing awareness of the associations of sedentary behaviour with increased mortality, of sedentary occupational time with biological markers of metabolic risk, and of the deleterious effects of pronged computer used, particularly repetitive strain injury (IJmker et al., 2007), the focus of increased occupational sitting and computer use and VTE events is less well explored. 


\section{The developing evidence for risk of venous thromboembolism with sedentary behaviours}

\section{Early case reports of venous thromboembolism associated with sitting}

The importance of seated immobility as a possible risk factor for VTE was made apparent in the early 1940's when Dr Keith Simpson, an English forensic pathologist recorded a sudden increased incidence of fatal PE (Simpson, 1940). He attributed this increase to prolonged periods of sitting in deckchairs while sheltering in bunkers during the wartime blitz. The rate of PEs was noted to decline when the chairs were replaced by stretchers which allowed shelter occupants to lay flat.

In 1952, Mr M Naide described six cases of tall men suffering VTE without prior surgery or other precipitating risk factors known at that time. In one case he described a 49 year old man who presented with a PE after a 3,000 mile drive, and in another, he presented a 42 year old lawyer who developed bilateral thrombophlebitis after horse-riding (Naide, 1952).

In 1954,Mr John Homans, a Boston surgeon described five cases in which protracted periods of sitting were implicated in the development VTE; a physician who undertook a 14 hour plane journey, a businessman who sat for many hours each day at his work desk, a young woman who drove for a protracted period of time in a car, a large older gentleman who undertook a holiday in his automobile, and a large gentleman who sat with his legs crossed at a theatre performance for two hours (Homans 1954). Homans proposed that the cases previously outlined by Mr Naide, and in his own described cases, prolonged sitting occasioned a 'degree of dependency stasis' that might result in the development of silent thrombosis in deep veins of the calf.

\section{Travellers ankle}

In 1973, Mr HD Johnson, a London surgeon proposed that 'travellers' ankle'; discomfort and swelling encountered by those who travelled by coach and plane, but not apparently by those who travelled by cars or trains, was caused by stasis in the wide sinusoidal veins of the calf. He postulated that ankle swelling was caused by raised intra-capillary pressure, approximately $60 \mathrm{mmHg}$ greater in the 'coach' position than the recumbent position. He 
advocated regular stretching to assist with resorption of the excess fluid, something which he proposed those who travelled by car and train were able to do (Johnson, 1973). He also proposed that post-operative and post-partum patients should strive to regularly exercise their feet and ankles to reduce the risk for VTE. Johnson commented that he felt that it might be difficult to prove or disprove his hypothesis as no single doctor was likely to have 'enough material for significant analysis'.

\section{Physiological changes and venous stasis with long distance travel}

There is clinical evidence that prolonged air travel increases Factor VII and Factor VIII activity and suppresses fibrinolysis in adults, and these changes occur in travellers who are considered both high risk and low risk for VTE (Wolfgang Schobersberger et al., 2003). This suggests that a degree of risk is conferred on all travellers due to their stasis, rather than preferentially isolating to travellers already considered at medium to high risk of VTE.

The same research group has also published findings from a study of 19 healthy volunteers who undertook a ten hour bus journey. They reported increased coagulation activity in these participants with an associated decrease in prothrombin time and activated partial thromboplastin time, and significantly increased calf volume. This suggests that a hypoxic aircraft cabin environment may not be a 'crucial' risk factor for VTE events after long flights; rather the seated component of prolonged travel may be more influential ( $\mathrm{W}$ Schobersberger et al., 2004).

In 2008 Hitos and colleagues undertook a series of different seating and exercise protocols in 21 healthy volunteers who were asked to sit in seats adjusted to replicate the height and pitch of major commercial airlines of the day (Qantas, British Airways and United economy passenger seats) (Hitos, Cannon, Cannon, Garth, \& Fletcher, 2007). When participants were sitting still with feet touching the floor for 100 minutes, popliteal blood flow velocity was reduced by a mean of $41 \%$ and blood volume flow was decreased by $42 \%$. When seat height was adjusted so that participants could not place their feet on the floor, blood volume flow was decreased by $48 \%$ over 95 minutes. These findings are consistent with the findings of the Cannegeiter and Kuiper case-control studies where shorter and taller people were associated with a higher risk of VTE with air travel. 


\section{Cases reports and case-control studies of travel-related venous thromboembolism}

In 1977, eight cases of PE after travel were described, three by car, three by plane, one by rail and one by rail/ship combined. All bar two of the patients had previously experienced venous disorders of the legs (Symington \& Stack, 1997). The authors proposed that stasis was the most likely culprit in these cases, due to relatively cramped seating arrangements, and this this situation was not necessarily limited to air travel. They also proposed venous stasis was contributory as well as pressure applied to the back of the leg, or sitting with legs crossed. The authors suggested regular changes in sitting position, good hydration, and exercises at rest to avoid stasis. Further case series were reported throughout the next two decades (Cruickshank, Gorlin, \& Jennett, 1988; Mercer \& Brown, 1998; Sarvesvaran, 1986).

A likely causal association between venous thromboembolism (VTE) and long distance air travel has since been elucidated. One of the first case-control studies in the medical literature reported that patients with VTE were four times more likely to have travelled for four or more hours via any form of transport within the four weeks prior to their index event (Ferrari, Chevallier, Chapelier, \& Baudouy, 1999). In the Travel-related analysis of the Multiple Environmental and Genetic Assessment of risk factors for venous thrombosis (MEGA) study, Cannegieter and colleagues analysed data from a pre-existing case-control study on risk factors for VTE in an unselected population in the Netherlands (S. C. Cannegieter et al., 2006). Travel greater than four hours, in the eight week period prior to the VTE index event was found to double the risk of VTE. The risk of flying was similar to travel by car, bus or train and in all instances was highest within one week of the travel being completed. Common additional risk factors in travellers who developed VTE were Factor $\mathrm{V}$ Leiden deficiency, $\mathrm{BMI}>30 \mathrm{~kg} / \mathrm{m}^{2}$, and being taller than $1.9 \mathrm{~m}$. An additional risk factor specifically for flying was a height shorter than $1.6 \mathrm{~m}$. Kuipers and colleagues subsequently reported additional risk factors for repeat travellers who developed VTE; women on the OCP, and short, tall or overweight individuals (Kuipers et al., 2007). 


\section{Air travel and venous thromboembolism}

In a review of risk factors for developing VTE associated with air travel, it was found that $72 \%$ of patients had one risk factor for VTE (excluding thrombophilia) before they flew. Many VTEs were associated with long trips with sequential flights (Kesteven \& Robinson, 2001).

Lapostolle and colleagues found in their review of all PEs at an airport in France between November 1993 and December 2000, that the incidence of PE was higher in passengers travelling more than $5,000 \mathrm{~km}$, compared to those travelling less than $5,000 \mathrm{~km}$. This finding was replicated in a study out of a major Madrid airport (Perez-Rodriguez et al., 2003). Additionally Lapostolle reported that the majority of VTE events had occurred in passengers who reported never having left their seats during the flight.

In a prospective, controlled cohort study, Schwarz and colleagues reported that those who undertook long-haul flights were twice as likely to have an isolated distal DVT (IDDVT) on ultrasound (US) examination, and were 4.4 times more likely to have a proximal DVT than those who did not travel (Schwarz et al., 2003).

At around the same time Hughes and colleagues reported a prospective study of 1,000 travellers undertaking an aircraft journey of $>4$ hours with a pre-travel d-dimer of $<500 \mathrm{ng} / \mathrm{L}$ (Hughes et al., 2003). Those who developed high-clinical probability symptoms, or who became D-dimer positive within three months after travel were investigated with bilateral compression US or computer tomographic pulmonary angiography (CTPA). 878 were included in the final analysis, with all participants travelling in excess of ten hours. 112 travellers met the criteria for further investigation, of which nine ( $1 \%$ of the total) had clinically proven VTE (four PE, 5 DVT). Of the nine VTE cases, five had been classified at enrolment as being at moderate or high risk of developing VTE using a standardised scoring system, and two had thrombophilic risk factors that were identified after travel. There was no difference in the duration of travel between those that developed VTE and those that did not. This study demonstrated that even in travellers who might be identified as low risk; there was an increased risk of VTE associated with long distance travel. 
Martinelli and colleagues undertook a case-control study of 210 patients who had experienced VTE and 210 healthy controls (Martinelli et al. 2003). They found that compared to controls, VTE cases were twice as likely to report air travel in the month prior to their VTE event. Those who undertook air travel and had a proven thrombophilia had a 16-fold increased risk of VTE, and women who undertook air travel and were on the OCP had a 14-fold increased risk of VTE. The authors went on to suggest that where risks are cumulative, simple measures such as hydration and leg exercises may not be enough, and that more aggressive VTE prevention methods might be required.

In France, Paganin and colleagues also reported a relationship between air travel and VTE, with $82 \%$ of VTE patients having other risk factors present. They did not find an association between oestrogen use and VTE, although they had only 27 female patients and 44 female controls. They did, however, find an association between VTE after travel and a previous history of VTE events, varicose veins, immobility during the flight and cardiac disease, and that complete immobility, and fewer times getting up were strongly associated with PE.

Belcaro and colleagues found in the Long Flights Thrombosis One (LONFLIT 1) study of 355 low-risk and 389 high-risk travellers, that low risk passengers had no VTE events after longhaul air travel, while $4.9 \%$ of high risk passengers had thrombotic events, including DVT and thrombophlebitis. In the LONFLIT 2 study, 833 passengers were randomised to wear graduated compression stockings and 411 to no intervention. In the stocking group $0.24 \%$ developed a VTE event, and $4.5 \%$ in the control group developed VTE (Belcaro, Geroulakos, Nicolaides, Myers, \& Winford, 2001).

\section{A contrary view}

In a cohort study of airline pilots, published in 2014, Kuipers and colleagues reported a standardised morbidity ratio of 0.8 for VTE in commercial airline pilots compared to the general Dutch population. When compared to a sample of employees of large organisations from a previous study (Kuipers et al. 2007), airline pilots had a standardised morbidity ratio of 0.7 . Not only this, but the incidence rate of VTE in pilots $(0.3 / 1,000$ person years) did not increase with cumulative flying hours or vary by rank. The phenomenon of better health outcomes in airline pilots is not new, probably due to the health related selection criteria for 
their job. Additionally they may enjoy less restriction of movement than their passengers, and finally, if VTE risk is at its highest closest to the initial point of exposure, it is possible that interviewing experienced pilots actually excludes pilots that may have developed VTE early on in their career and have since left the profession.

In the now numerous studies investigating VTE risk associated specifically with air travel, the predominant theme arising is that the risk of VTE is greatly increased when in conjunction with one or more other risk factors (known or consequently diagnosed) for VTE. Additionally many studies have found increasing risk of VTE with increasing frequency and duration of travel, raising the possibility that it is 'too much sitting' rather than 'too much flying' that is the underlying contributory risk factor in this clinical setting (Dalen 2003).

\section{Evidence of venous thromboembolism associated with sedentary behaviours}

More recently in a prospective cohort study of nearly 69,950 nurses who completed biennial questionnaires that included questions on physical activity and sedentary behaviours, it was found that there was an association between time spent sitting and risk of 'idiopathic' PE (no history of surgery or major trauma within one month of the diagnosis, and no history of active malignancy), with a two-fold risk of PE in women who spent the most time sitting compared to those who spent the least time sitting (Kabrhel, Varraso, Goldhaber, Rimm, \& Camargo Jr., 2011). If this association is true, then consideration needs to be given to how to mitigate this risk in environments where sitting for prolonged periods occurs, particularly sedentary work roles and computer use both for work and for recreational activities.

In 2016, Shirakawa and colleagues reported increasing risk of PE as television viewing hours per day increased in a population-based cohort study involving 110,585 participants and calculated that the mortality rate from PE was 8.2 per 100,000 person-years among those watching television $\geq 5$ hours per day (Shirakawa et al. 2016). 


\section{Occupational sitting, computer use and venous thromboembolism}

Clinical evidence to support the biological plausibility of prolonged seated immobility as a risk factor for VTE in the working environment was provided by Levin and colleagues in 2009. They assessed popliteal venous flow of adults sitting in ergonomic office chairs as if they were in an office environment (Levin, Macintosh, Baker, Weatherall, \& Beasley, 2008). Levin found that compared to popliteal venous blood flow in the prone position, PSV in the seated position with legs flexed at 90 degrees fell by more than $80 \%$ after five minutes, with a reduction in total blood flow in excess of $70 \%$. These results were similar when sitting with legs flexed at 120 degrees. This provided clinical evidence of a substantial reduction in venous flow very early after assuming the standard seated position at a work desk.

\section{Case reports of venous thromboembolism after prolonged work- or computer-related sitting}

In 2003 Beasley and colleagues reported a case of a 32 year old male who presented with shortness of breath at rest, preceded by a six-week period of a swollen painful calf, which resolved after ten days. He was diagnosed with a PE and started on anticoagulation. A thrombophilia screen was normal, and no other recognised risk factors for VTE were found. He reported sitting in front of his computer for work and recreation, between 12 and 18 hours per day, and for up to six hours at a time without getting up. Beasley proposed the term 'eThrombosis' to describe this phenomenon and recommended research to estimate the risk of VTE associated with sitting at computers for work and recreation (Beasley, Raymond, et al. 2003).

Beasley described a second similar case the same year. A 53 year old woman spent three hours watching a move in the local movie theatre and developed sudden pain in her right leg soon after leaving. A popliteal DVT was diagnosed and she started anticoagulation therapy. In this instance, the patient had a DVT 27 years prior, which was considered provoked by the use of the OCP and had since fully resolved. In this instance she described episodes of prolonged computer use, where she would regularly sit for two to three hours at a time without getting up. Taking up the common theme of sitting, whether it be the already well known risk of air travel, at a movie theatre or working with computers, Beasley 
proposed use of the term 'seated immobility thromboembolism (SIT) syndrome' to encourage clinicians to consider all forms of protracted immobility when assessing risk for VTE (Beasley, Heuser, et al. 2003).

In 2004 Ho Lee, in Korea described a case of a 34 year old male who died from a PE after 80 hours of computer use. New bilateral popliteal thrombi were found posthumously. No other recognised risk factor for VTE was found. At that time in Korea, every child over the age of six accessed the internet, and the mean weekly computer use for all Koreans was 12.2 hours. The author concluded that computer use in Korea would place their younger population at risk of eThrombosis (H. Lee, 2004).

Beasley went on to describe a further five cases of VTE in younger people associated with prolonged sitting at work or computer use. Each of the cases were under 40 years of age, and described sitting for eight to 15 hours per day sitting for work or recreation, with a maximum of ranging between eight and 30 hours. All of them reported sitting for between three and six hours at a time without getting up. None of the cases had any other identified risk factor for VTE. Of interest, one case, a computer programmer, developed a DVT in the axillary and subclavian veins while the remaining four cases had proximal DVT and / or PE (Beasley, Heuser, \& Raymond, 2005).

Elikowski and colleagues described six different cases of individuals who had suffered VTE provoked by prolonged sitting at computers. Four of them were 30 years of age or younger, and had described spending between 12 and 16 hours a day in front of the computer, and sitting continuously for three to five hours at a time before getting up (Elikowski et al. 2011).

\section{Case reports of venous thromboembolism with extended gaming}

Reported cases of VTE after prolonged episodes of online gaming have followed a similar trajectory to reports of prolonged work- and computer-related sitting. Of note some VTE events have been reported in very young patients.

In 2003, Ng, a paediatric Registrar in the UK described the occurrence of a DVT in a 12 year old child who had spent the day prior to presentation kneeling on his lower legs while using a game console to play a computer game. The child had been kneeling in this position for up 
to four hours without a break. Thrombophilia screen after diagnosis was normal. This case is of particular interest as it occurred in a child outside of the usual context of malignancy, surgery or thrombophilia, and perhaps in the context of what is becoming an increasingly normal behaviour in young children; mechanical compression of the legs while seated for prolonged periods of time playing computer or console games $(\mathrm{Ng}$, Khurana, Yeang, Hughes, \& Manning, 2003).

In Singapore in 2006 a 16 year old boy presented with bilateral DVTs and a PE after a three hour session playing computer games. He had a history of a PE the year before, also preceded by prolonged sessions playing computer games. His coagulation screen was normal. He underwent surgical removal of his embolism (Chew, 2006).

In 2013 Chang and colleagues described a case of a New Zealand man who spent seven to eight hours each day gaming while he sat on his bed with his legs in front of him. This patient presented with a DVT that extended into his femoral vein and his common iliac vein, with features of occlusion, for which he underwent thrombolysis. He had no other recognised risk factors for VTE and his thrombophilia screen was normal (Chang, Burbridge, \& Wong, 2013).

In conjunction with my colleagues in 2014 , I reported a case of a 34 year old man who had been online 'gaming' for 48 hours prior to a collapse that lead to him presenting to hospital where he was diagnosed with a PE. As well as his online gaming, he had the additional features of morbid obesity and Klinefelters syndrome that in themselves may have some contributory risk for VTE. He described playing online computer games for up to nine days at a time with minimal food and hydration, and sleeping in his computer chair for only short periods so that he would not miss out on his turn in the computer game (Braithwaite et al. 2014).

Finally, Ken Lee described a fatal episode of VTE when a 16 year old youth diagnosed with internet addiction, spending more than 13 hours playing on his computer each day, presented with a DVT and massive PE. A thrombophilia screen was negative and no other risks for VTE were found. He was started on anticoagulation. Six months later, shortly after 
completing his treatment, he developed a life-threatening PE for which he underwent thrombolysis. He developed cardiovascular collapse and had to undergo an emergency pulmonary thrombectomy. He then had an inferior vena cava (IVC) filter placed as well as being prescribed anticoagulation. After two years of treatment, complete radiological resolution of the PE and DVTs was confirmed and his anticoagulation was stopped but his IVC filter remained in situ. A repeat thrombophilia screen was negative. He was advised to avoid prolonged immobility, but no formal counselling or psychological assessment was made of his continued and persistent computer use despite two near-fatal VTE events. Nine months later he suffered a fatal massive PE. This case illustrates the need to modify sedentary behaviour, and to seek assistance for this if needed, once it has been implicated as a risk factor for VTE (K. Lee, Lim, \& Hsu, 2015).

\section{A case series of VTE and work-and computer-related immobility}

In 2008, Aldington published a case series of adult patients aged no more than 65 years who had attended Wellington Hospital VTE outpatient clinic for follow-up after a diagnosis of DVT or PE within the previous 12 months (Aldington et al., 2008). 61 patients who had been diagnosed with a VTE event answered a questionnaire that included questions about usual risk factors for VTE and any episodes of prolonged seated immobility at work that may have occurred in the four weeks prior to their index event. Of the 61 respondents, $34 \%$ indicated that in the four weeks prior to presentation, they had met one of three possible definitions of prolonged seated immobility at work; at least 8 hours of sitting in a 24 hour period, with at least a three hour period without getting up, at least 10 hours of sitting in a 24 hour period with at least a two hour period without getting up, or sitting at least 12 hours in a 24 hour period with a period of at least one hour without getting up. These initial criteria of prolonged seated immobility at work were estimated based on the results of previous casecontrol studies of air travel, where VTE events had been strongly associated with longer flights, and with longer periods of immobility during the flights (Lapostolle et al., 2001; Paganin et al., 2003). As there was no control group the odds ratio for association of prolonged seated immobility at work with VTE could not be calculated. 
Even in conjunction with physiological studies showing reduced blood flow while sitting in office chairs, multiple case studies and a case series of VTE events cannot demonstrate an association with VTE. The relatively young age of many of these cases where the risk factor of immobility is modifiable, makes it desirable to demonstrate an association if one exists. This would provide an evidence base for interventions that could reduce the morbidity and mortality associated with these VTE events. The background rate of VTE is very low (as already discussed earlier in this chapter), so a randomised controlled trial of an intervention to reduce sedentariness at work and with computer use with VTE as an outcome is unlikely to be viable. With this limitation, a case -control study would seem an appropriate way to assess the relationship of seated immobility with VTE.

\section{Two case-control studies: work- and computer-related immobility}

Using the proportion of $34 \%$ of VTE patients describing a recent history of prolonged seated immobility at work that Aldington had reported, West and colleagues published the first case-control study investigating the association between prolonged seated immobility at work and VTE (West et al. 2008). Included cases consisted of 97 patients aged 18 to 65 years who attended the Wellington Hospital VTE Clinic following a radiologically confirmed VTE diagnosis. The controls comprised 106 patients aged 18 to 65 years admitted to the coronary care unit (CCU) at Wellington Hospital. West used the same variables as Aldington $(8+3,10+2,12+1)$ to define prolonged seated immobility.

The proportion of VTE patients that reported prolonged seated immobility at work in this study was $21 \%$ rather than the $34 \%$ reported in the case series, thus the study was slightly underpowered. The univariate and multivariate odds ratios for the association between prolonged seated immobility at work were calculated. In the univariate analysis, prolonged seated immobility at work was associated with a 2-fold increased risk of VTE, and prolonged seated immobility at work AND at home in front of the computer (TOTAL prolonged seated immobility) was associated with a nearly 3-fold increased risk of VTE. When included in a multivariate analysis with age, family history of VTE, previous history of VTE, surgery or trauma in the four weeks prior to the index event, and travel greater than four hours prior to the index event, prolonged seated immobility at work was associated with a 1.8-fold 
increased risk of VTE, and total prolonged seated immobility was associated with a 2.2-fold increased risk of VTE, but the associations were no longer significant.

There was a consistent association between the risk of VTE and all four parameters of maximum sitting time investigated; maximum hours seated in total in a 24 hour period, maximum hours seated only for work in a 24 hour period, the longest period seated in total without getting up and the longest period seated for work only without getting up.

West and colleagues concluded that here was preliminary evidence that prolonged sitting was associated with VTE. The selection of controls from the CCU may have influenced the result, as they were more likely to be on anticoagulants as part of their routine cardiology care and may have had had more sedentary roles due to the presence of cardiology symptoms. The suggestion of increased VTE risk with prolonged seated immobility at work, and total prolonged seated immobility, and the increase in risk associated with each additional hour of sitting time without getting up were consistent with results reported in studies of VTE and travel-related risk (Lapostolle et al., 2001; Paganin et al., 2003).

A second, larger, case-control study was undertaken, which as well as exploring maximum sitting times, also considered average time seated, specific occupational roles, desk and seat arrangements and workplace habits such as leaving the desk to eat lunch (B. Healy, Levin, et al., 2010). The 197 cases again comprised of patients aged 18 to 65 years attending VTE outpatient clinics at Wellington Hospital with a confirmed diagnosis of VTE in the previous six months. The six month period was chosen in an attempt to limit recall bias that might have occurred in the first study. The 197 controls were recruited from the Wellington Hospital CCU, and where possible they were age and sex matched with the VTE cases. In this case-control study, prolonged work- and computer-related seated immobility was defined as 10 hours of sitting during a 24 hour period, either at work or in front of a computer at home, and a period of at least two hours sitting without getting up. This criterion had to be met at least once in the four weeks prior to their index event. The $10+2$ variable was directly derived from Aldington and West, and represented the variable with the best area under the receiver operating curve for predicting risk of VTE, with a c-statistic of 0.565 . This case-control study differed somewhat from that in the West study in that it included work- 
related habits such as whether lunch was eaten at the desk or not, desk and chair seating arrangements and information related specifically to occupational roles.

In this study, the proportion of cases meeting the definition of prolonged work- and computer-related seated immobility was $17 \%$, and in controls it was $10 \%$. In multivariate analysis, prolonged work and computer-related seated immobility was associated with a significant 2.8-fold increased risk of VTE. Consistent with the Aldington study, there was a $10 \%$ increased risk of VTE with each hour increase in maximum sitting time and with each hour increase in average sitting time in a 24 hour period. There was a suggestion of increased risk with every extra hour of sitting without getting up.

In considering occupational categories, there was a suggestion that 'clerical / administration workers' and 'professionals' might be disproportionately represented in the cases. The selection of cases from the CCU of Wellington Hospital was based on the understanding the VTE and arterial thrombosis shared some common risk factors, but if patients had symptomatic cardiovascular disease, they may have been over-represented in sedentary occupations and the odds ratio for VTE due to prolonged work- and computer-related seated immobility may have been understated.

\section{Third case-control study for this thesis}

As part of this thesis, I undertook a third case-control study comparing work and computer related sitting behaviours and VTE risk factors between 200 VTE patients attending VTE outpatient clinics at Wellington Hospital (cases) and 200 outpatients at the Fracture Clinic of Wellington Regional Hospital who had no history of VTE events within the six months prior to their presentation with their index fracture event (controls).

The reasons for selecting controls from outside of the CCU setting were three-fold; firstly, this fracture clinic control group would be more likely to have a smaller proportion of individuals taking antithrombotic medication than those with diagnosed cardiovascular conditions, secondly, it might be less likely that controls would be undertaking sedentary roles due to any pre-existing cardiovascular medical conditions that might limit their activity, thirdly, this control group might have a lower concentration of the VTE risk factors 
that have been shown to be common between VTE patients and those with cardiovascular disease. I also gathered data on laptop use, both at work and at home and the position the laptop was used in (sitting and / or on lap) to ensure that all computer use was captured and to see if additive laptop and desktop use conferred additional risk for VTE. 


\section{STUDY ONE: Venous thromboembolism risk associated with protracted work- and computer-related seated immobility: A case-control study}

\section{Hypothesis}

The hypothesis of this study was that prolonged work- and computer-related seated immobility defined as being seated at work, and/or on the computer at home for at least 10 hours within a 24-hour period and, within that same period, for at least 2 hours without getting up, is associated with an increased risk of VTE.

\section{Primary Outcome Variable}

The primary outcome variable was the odds ratio for association with VTE of prolonged work- and computer-related seated immobility.

\section{Secondary Outcome Variables}

Secondary outcome variables were the odds ratio for association of VTE with increasing mean hours seated during a working day and the odds ratio for association with VTE with increasing maximum hours seated during a working day.

\section{Method}

This was a case-control study of 200 patients (cases) attending VTE clinics with a history of radiologically confirmed VTE in the past six months and 200 patients (controls) treated in fracture clinic for an upper limb injury with no history of VTE in the preceding six months. Univariate logistic regression was used to measure the strength of the association between case-control status and other explanatory variables. For the continuous variables the difference in mean values was also assessed with a simple t-test.

\section{Note}

This study has been reproduced in accordance with the publication permissions of the Journal of the Royal Society of Medicine, Sage Journals (I Braithwaite, Maher, Cameron, Weatherall, \& Beasley, 2016). Permissions are included in Appendix XII. The discussion section has been augmented to include an updated literature review. 


\section{Methods}

Cases for the study were from consecutive patients attending the Capital and Coast District Health Board (CCDHB) VTE service outpatient clinics between October 2011 and January 2013. For inclusion as a case the patients needed to be aged between 18 and 65 years and have a confirmed diagnosis of VTE within the last six months. With the exception of oncology patients, the VTE Service sees most cases who have presented for diagnosis and management in both primary and secondary healthcare services. A diagnosis of VTE required confirmation by compression Doppler Ultrasound, lung ventilation / perfusion scan, or helical CTPA. Patients with arterial thromboembolic disease or who had superficial thrombophlebitis without extension into the deep venous system were excluded.

Controls were recruited from orthopaedic outpatient departments at CCDHB between February 2011 and April 2013. Patients were eligible if they were aged between 18 and 65 years of age, had suffered a traumatic upper limb injury within the previous six months and had no VTE, or clinical suspicion of VTE, within the same time frame. The choice of upper limb injuries as a control group was made as this patient group would come from within the same community as the VTE cases, and similar to the VTE cases, would present to the same local emergency department with an acute event and be managed within an inpatient setting if indicated, with follow-up care provided in an outpatient setting.

Cases and controls were matched by sex and age within five years. Both cases and control participants completed an interviewer-administered questionnaire (Appendix II, Appendix III) about demographic data, occupational status, clinical details of the index case or control event, VTE risk factors, working environment and sports activities. VTE risk factors were grouped into categories shown in Table 2.1. 
Table 2.1 Risk factors for venous thromboembolism

$1 \quad$ Age

2 Sex

3 Obesity $\left(\mathrm{BMI}>30 \mathrm{~kg} / \mathrm{m}^{2}\right)$

4 Personal history of VTE - confirmed previous VTE event $>6$ months prior to inclusion in this study

5 Family history of VTE - confirmed VTE in parents or siblings

6 Long distance travel in the 4 weeks prior to presentation

- Air travel: any international flights ${ }^{\dagger}$

- Road-based travel greater than 4 hours (car, bus, motorbike, camper-van)

7 Surgery requiring general anaesthetic or spinal/epidural anaesthesia in the 4 weeks preceding presentation

8 Medical Immobility - general acute or chronic immobility for greater than 2 days' duration, other than post-operatively

- Includes bed-rest, wheelchair use over the previous 4 weeks, and overdose with reduced level of consciousness

9 Trauma or cast immobility in the four weeks preceding presentation

- Trauma requiring medical attention

- Cast immobility - orthopaedic limb/foot cast

10 Pregnancy or hormonal treatment, including hormonal contraceptive, hormone replacement therapy, or hormonal antagonist therapy for malignancy

11 Active malignancy or chronic medical illness known to pre-dispose to VTE, such as inflammatory bowel disease, cardiomyopathy or congestive heart failure, connective tissue disease including inflammatory arthritis

12 Smoking in the four weeks prior to presentation

13 Personal history of varicose veins, whether treated or not

14 Prolonged work- and computer-related seated immobility

- $\quad$ the duration seated at work and at home seated in front of a computer in the 4 weeks prior to presentation, defined as seated for at least 10 hours in a 24 hour period and at least 2 hours at a time without getting up, at least once in the 4 weeks before presentation

15 Strenuous exercise - for greater than 30 minutes at a time at least once in the 4 week prior to presentation

BMI - Body mass index

VTE - Venous thromboembolism

† International flights: from New Zealand this is equivalent to sitting in a plane for at least 4 hours 
Prolonged work- and computer-related seated immobility was defined in two ways. The first was as a categorical variable; as being seated at work, and/or on the computer at home for at least 10 hours within a 24 -hour period and, within that same period, for at least 2 hours without getting up. Subjects had to do this at least once in the 28 days before the onset of symptoms in the index event. If participants drove for their occupation (e.g. truck or taxi driver, courier), this was included in the immobility calculation, but commuting to and from work was not included. Secondly work- and computer-related seated immobility was also measured as continuous variables including the mean and maximum number of hours seated in a 24-hour period and the maximum number of hours seated at one time without getting up. This was also for the 28 days before the onset of symptoms in the index event.

Occupation was categorized according to Australia and New Zealand Standard Classification of Occupations (ANZSCO) (Appendix IV) then combined into high and low sedentary categories as previously described (B. Healy, Levin, et al., 2010). Medical records for the cases were reviewed for radiological confirmation of VTE, and to assess whether seated immobility for travel or at work were listed as risk factors by the referring medical professional and/or the assessing doctor at presentation. Data collection also included whether the VTE event was considered to be idiopathic, whether a thrombophilia screen had been requested, and any subsequent results.

\section{Statistical Methods}

Univariate logistic regression was used to measure the strength of the association between case - control status and other explanatory variables. For the continuous variables the difference in mean values was also assessed with a simple t-test. In a multivariate logistic regression, the predictor variables for case-control status were: one or more episodes of prolonged work- and computer-related immobility in the 28 days prior to the index event, maximum hours using a computer in one day, average hours of computer use in one day, and maximum and average hours using a laptop in one day. These were adjusted for prespecified confounding variables: $\mathrm{BMI}>30 \mathrm{~kg} / \mathrm{m}^{2}$, a personal history of VTE, a family history of VTE, and any of the following within the 28 days prior to the development of symptoms of 
the index event; immobility, surgery, cast immobilisation, air travel and/or road travel, and strenuous exercise in the 28 day period prior to the index event.

\section{Power Calculation}

Based on previous studies (B. Healy, Levin, et al., 2010; West et al., 2008) I anticipated that $10-15 \%$ of controls would meet the categorical definition for prolonged work- and computer-related seated immobility. It was anticipated that a sample size of 400 (200 cases and 200 controls) would give $80 \%$ power to detect an odds ratio for risk of 2.0 if the control rate was $15 \%$, and an odds ratio for risk of 2.25 if the control rate were $10 \%$, with a type 1 error rate of $5 \%$. These odds ratios were based on the previous studies in which similar categorical variables for prolonged work- and computer-related seated immobility were associated with VTE, with odds ratios of 1.8 (95\% Cl 0.71 to 4.8 ) (West et al., 2008) and 2.8 (95\% Cl 1.2 to 6.1, P=0.013) (B. Healy, Levin, et al., 2010).

SAS version 9.3 was used. 


\section{Results}

Of 206 possible cases approached to take part in the study four declined and two were excluded as they did not have radiologically confirmed DVT or PE, leaving 200 cases for analysis. The mean age (SD) of the cases was 46.1 (12.7) years and 93 were female. There were 136 index DVTs; 121 in the lower limb (53 proximal, 67 distal and one unspecified), 14 in the upper limb and one abdominal. There were 71 cases of pulmonary embolism (seven of whom also had confirmed DVT); 14 were sub-massive (large thrombus burden with a troponin T rise or evidence of right ventricular strain), and four were massive (large thrombus burden with syncope and/or systolic blood pressure less than $90 \mathrm{mmHg}$ ).

Of 250 possible controls approached; 27 declined and 23 were unable to be matched with the cases, leaving 200 age and sex-matched controls. The mean (SD) age of the controls was 45.9 (13.1) years and there were 93 females. There were 154 fractures, 15 sprains, 31 'other' injuries (e.g. dislocation, laceration). Most injuries (58\%) were distal.

The univariate analysis of the association between the different risk factors and VTE is shown in Table 2.2. Increased risk of VTE was observed with recognised risk factors including personal or family history of VTE, medical-related immobility, pregnancy or hormone therapy, surgery, trauma or cast immobility. For travel, road travel but not international air travel was associated with an increase in VTE risk, with odds ratios of 1.80 (95\% $\mathrm{Cl} 1.10$ to 2.95$) \mathrm{P}=0.02$ and 1.75 (95\% $\mathrm{Cl} 0.93$ to 3.32 ) $\mathrm{P}=0.085$ respectively. There was no significant association between prolonged work- and computer-related seated immobility and VTE risk, odds ratio 1.20 (95\% $\mathrm{Cl} 0.71$ to 2.03$) \mathrm{P}=0.50$. 
Table 2.2: Characteristics of study participants and univariate odds ratios between potential risk factors and venous thromboembolism.

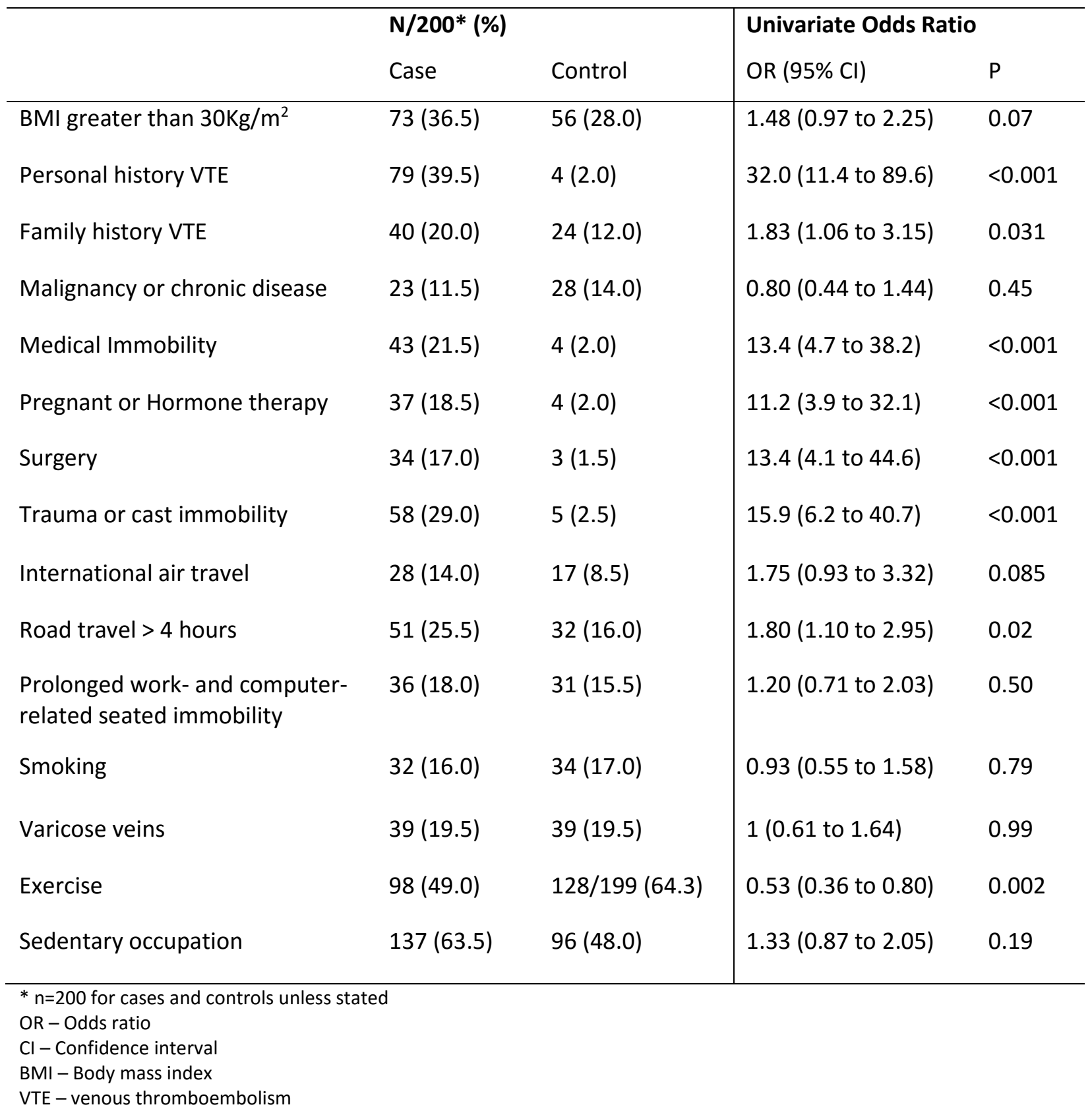


The univariate and adjusted multivariate associations between VTE and both categorical and continuous measures of work- and computer-related seated immobility are shown in Table 2.3. In the multivariate analyses, prolonged work- and computer-related seated immobility expressed as a categorical variable was not associated with VTE with an odds ratio of 1.18 (95\% $\mathrm{Cl} 0.56$ to 2.48 ) $\mathrm{P}=0.67$. As a continuous variable, each one hour increase in mean hours seated within a 24 hour period was associated with VTE with an odds ratio of 1.08 (95\% $\mathrm{Cl} 1.01$ to 1.60 ) $\mathrm{P}=0.023$. For travel, international air travel but not road travel was associated with an increase in VTE risk, with odds ratio of $2.6(95 \% \mathrm{Cl} 1.12$ to 6.3$) \mathrm{P}=0.026$, and $1.6(95 \% \mathrm{Cl} 0.77$ to 3.32$) \mathrm{P}=0.21$ respectively in the multivariate analyses. 
Table 2.3: Univariate and multivariate associations between components of seated immobility and VTE event.

\begin{tabular}{|c|c|c|c|c|}
\hline & \multicolumn{2}{|c|}{ Mean (SD) } & \multicolumn{2}{|c|}{$\begin{array}{c}\text { OR } \\
\text { (95\% Cl) } \\
\text { P value }\end{array}$} \\
\hline & Case & Control & Univariate & Multivariate* \\
\hline $\begin{array}{l}\text { Mean work/computer } \\
\text { seated (per hour) }\end{array}$ & $5.5(3.8)$ & $4.6(3.9)$ & $\begin{array}{l}1.06 \\
(1.01 \text { to } 1.12) \\
0.02\end{array}$ & $\begin{array}{l}1.08 \\
(1.01 \text { to } 1.6) \\
0.02\end{array}$ \\
\hline $\begin{array}{l}\text { Max work/computer } \\
\text { seated without getting } \\
\text { up (per hour) }\end{array}$ & $2.9(2.1)$ & $2.6(2.4)$ & $\begin{array}{l}1.07 \\
(0.98 \text { to } 1.16) \\
0.16\end{array}$ & $\begin{array}{l}1.10 \\
(0.98 \text { to } 1.23) \\
0.11\end{array}$ \\
\hline $\begin{array}{l}\text { Mean laptop on lap (per } \\
\text { hour) }\end{array}$ & $0.6(1.5)$ & $0.4(1.2)$ & $\begin{array}{l}1.09 \\
(0.94 \text { to } 1.27) \\
0.25\end{array}$ & $\begin{array}{l}1.16 \\
(0.95 \text { to } 1.41) \\
0.15\end{array}$ \\
\hline \multirow[t]{3}{*}{$\begin{array}{l}\text { Max laptop on lap (per } \\
\text { hour) }\end{array}$} & $0.9(2.3)$ & $0.8(1.7)$ & $\begin{array}{l}1.05 \\
(0.95 \text { to } 1.16) \\
0.33\end{array}$ & $\begin{array}{l}1.09 \\
(0.96 \text { to } 1.24) \\
0.21\end{array}$ \\
\hline & $N / 200(\%$ & & & \\
\hline & Case & Control & & \\
\hline $\begin{array}{l}\text { Prolonged work- and } \\
\text { computer-related } \\
\text { seated immobility }{ }^{\dagger}\end{array}$ & $36(18.0)$ & $31(15.5)$ & $\begin{array}{l}1.20 \\
(0.71 \text { to } 2.03) \\
0.50\end{array}$ & $\begin{array}{l}1.18 \\
(0.56 \text { to } 2.48) \\
0.67\end{array}$ \\
\hline
\end{tabular}

*All adjusted for BMI greater than 30, personal history of VTE, family history of VTE, Immobility, surgery, use of cast, recent air travel, recent road travel, and exercise status

+ Prolonged work- and computer-related seated immobility - the duration seated at work and at home seated in front of a computer in the 4 weeks prior to presentation, defined as seated for at least 10 hours in a 24 hour period and at least 2 hours at a time without getting up, at least once in the 4 weeks before presentation

VTE - Venous thromboembolism

$\mathrm{SD}$ - standard deviation, OR - Odds ratio, $\mathrm{Cl}$ - Confidence interval 
Table 2.4 shows the occupational groups of cases and controls. Sedentary occupations (managers, professionals, clerical and administrative workers) comprised $68.5 \%$ of cases and $48 \%$ of controls. Amongst the cases and controls, women were not more likely to be employed in sedentary occupations than men, with 55 of 137 (40\%) and 47 of 96 (49\%) of sedentary roles occupied by women in the cases and controls respectively. However, within sedentary occupations, women were more likely than men to be clerical and administrative workers ( 17 of 29 cases and 14 of 20 controls) and men were more likely to be managers and professionals ( 70 of 108 cases and 43 of 76 controls). There was no significant association between VTE and sedentary occupation with odds ratio of 1.33 ( $95 \% \mathrm{Cl} 0.87$ to 2.05) $\mathrm{P}=0.19$. 
Table 2.4: Study participants by occupational group and sex, $\mathbf{N}=\mathbf{2 0 0}$ for both cases and controls

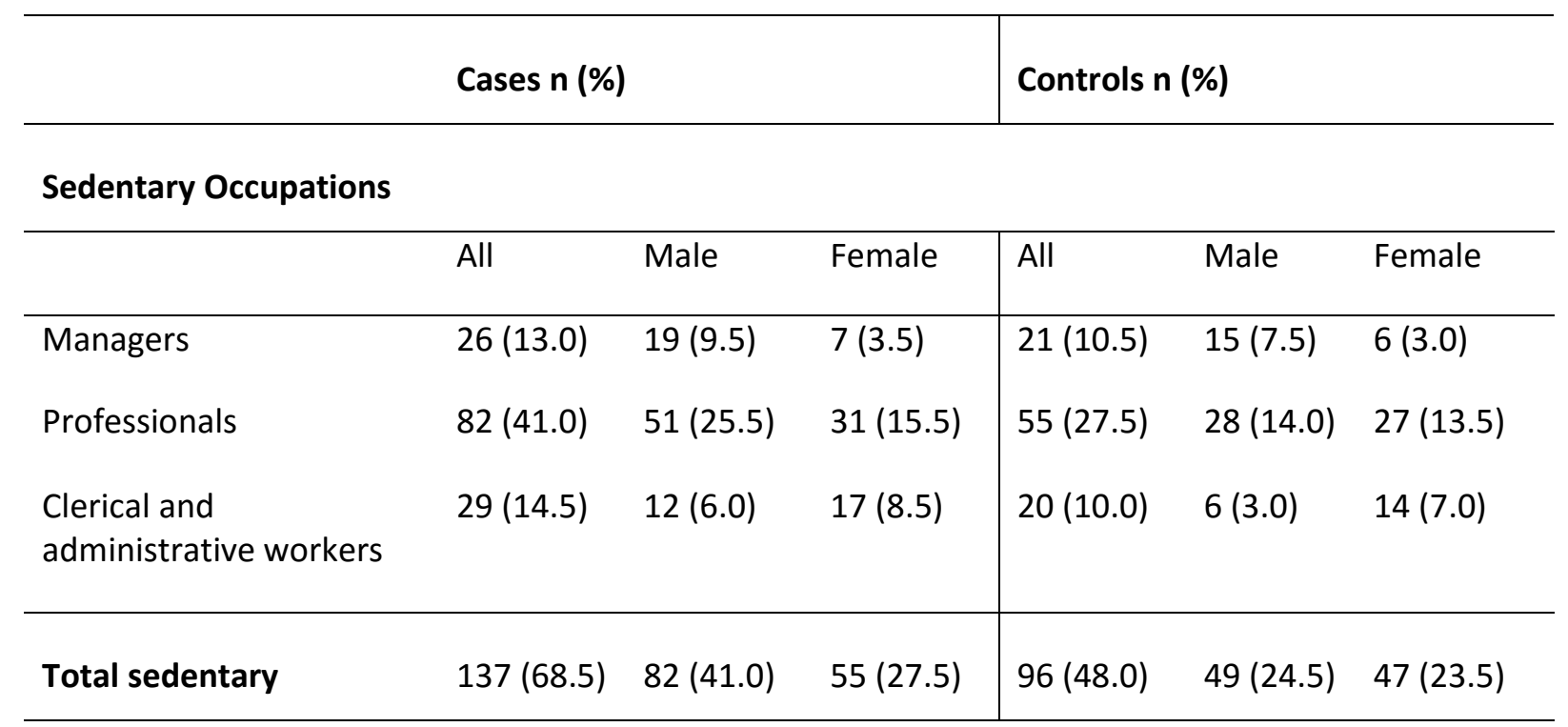

Non-sedentary Occupations

\begin{tabular}{|c|c|c|c|c|c|c|}
\hline & All & Male & Female & All & Male & Female \\
\hline $\begin{array}{l}\text { Technicians and trade } \\
\text { workers }\end{array}$ & $9(4.5)$ & $6(3.0)$ & $3(1.5)$ & $22(11.0)$ & $18(9.0)$ & $4(2.0)$ \\
\hline $\begin{array}{l}\text { Community and personal } \\
\text { service workers }\end{array}$ & $6(3.0)$ & $1(0.5)$ & $5(2.5)$ & $16(8.0)$ & $4(2.0)$ & $12(6.0)$ \\
\hline Sales workers & $6(3.0)$ & $2(1.0)$ & $4(2.0)$ & $11(5.5)$ & $4(2.0)$ & $7(3.5)$ \\
\hline $\begin{array}{l}\text { Machinery operators and } \\
\text { drivers }\end{array}$ & $1(0.5)$ & $1(0.5)$ & - & $8(4.0)$ & $8(4.0)$ & - \\
\hline Labourers & $8(4.0)$ & $4(2.0)$ & $4(2.0)$ & $11(5.5)$ & $11(5.5)$ & - \\
\hline Unemployed & $33(16.5)$ & $23(11.5)$ & $10(5.0)$ & $36(18.0)$ & $14(7.0)$ & $22(11.0)$ \\
\hline Total non-sedentary & $63(31.5)$ & $37(18.5)$ & $26(13.0)$ & $104(52.0)$ & $59(29.5)$ & $45(22.5)$ \\
\hline
\end{tabular}




\section{Discussion}

This case-control study has found a weak association between prolonged work- and computer-related seated immobility and VTE risk. Although the point estimates for all the categorical and continuous variables were consistent with an increased risk, only the measurement of seated immobility defined as the mean number of hours seated in a 24 hour period was statistically significant, with the odds ratio for risk increasing by 1.08 for each one hour longer seated in a 24 hour period.

These findings complement the previous case-control studies undertaken by West and Healy, which utilised similar criteria for prolonged work- and computer-related seated immobility, but a different control group (B. Healy, Levin, et al., 2010; West et al., 2008). In the previous studies controls were selected from patients presenting to $\mathrm{CCU}$, based on the landmark case-control study on traveller's thrombosis (Ferrari et al. 1999) and recognition of common risk factors for VTE and atherosclerosis (B. Healy, Levin, et al., 2010; Kuipers et al., 2014). I hypothesised that this CCU group might result in an underestimation of the risk associated with prolonged seated immobility as such patients may be more likely to have a sedentary occupation due to limitations placed on them by their cardiovascular disease. For this reason I chose a different control group, a 'healthy' group presenting to the Emergency Department with upper limb injury in which there was no clinical suspicion of VTE. In addition to age, I also matched by sex in the current study, due to the possibility that women were more likely to be in sedentary employment. This did not turn out to be the case with a similar proportion of men and women reporting a sedentary occupation in the control group.

In this study, using fracture clinic patients as the control group resulted in a greater proportion of controls having prolonged work- and computer-related seated immobility than in the previous study with the CCU-derived controls $(15.5 \%$ vs $9.6 \%$ in fracture controls and CCU controls respectively). In contrast, the proportion of VTE cases reporting prolonged work- and computer-related seated immobility was similar ( $18.0 \%$ vs $16.8 \%$ respectively). The use of the fracture clinic control group may have unexpectedly been inappropriate, as this group may have an association with immobility and their risk of upper limb fracture, 
perhaps related to reduced bone density secondary to immobility, or a greater tendency to fall and injure themselves because of less physical fitness and balance. It is possible the selection of controls derived from CCU may be preferable, on the basis that it may be more representative of the population at risk of presentation to hospital with a venous thrombosis (B. Healy, Levin, et al., 2010; Kuipers et al., 2014).

It seems likely that although convenient to treat seated immobility as a dichotomous variable, this may lose information relevant to a causal relationship between this behaviour and VTE, despite its' apparently strong association with VTE risk in the Healy study.

Dichotomising continuous variables can lead to a lack of power, and can increase the risk of Type 1 error in association studies. I anticipated this might be the case and, as West and Healy had done, I also examined continuous variables relating to the total duration of being seated and the duration seated at a particular time, both of which contribute to the VTE risk with prolonged seated immobility during long distance air travel (Lapostolle et al., 2001; Perez-Rodriguez et al., 2003). These included the association with the mean duration of seated immobility, in addition to the maximum duration on a single occasion, to obtain a measure of the burden of seated immobility over a 28 day period. Also, I examined the association with laptop use, in addition to computer use while seated. For these continuous variables, the point estimates all favoured an association between measures of prolonged seated immobility and VTE risk, although only the mean number of hours seated in a 24 hour period was statistically significant.

I assessed the role of seated immobility at work by crude classification of individuals into high and low sedentary occupations. Sedentary occupations, including managers, professionals (including information technology) and clerical and administrative workers, had a point estimate of association of an odds ratio of association with VTE of 1.3 compared to non-sedentary occupations. This is consistent with previous finding of an odds ratio for association of with VTE of 1.7 in the same sedentary occupational groups (B. Healy, Levin, et al., 2010). However, the association was not significant after adjusting for other confounding variables including prolonged work- and computer- related seated immobility at work and at home. This suggests that prolonged sitting rather than other aspects of an 
occupation underlies the association and is consistent with the findings of a Danish cohort study based on national registers that reported that drivers potentially exposed to prolonged cramped sitting at work were at a 1.28-fold greater risk of pulmonary embolism than a socioeconomically comparable group of construction workers with more dynamic physical work demands (Suadicani, Hannerz, Bach, \& Gyntelberg, 2012). It is unclear why the incidence of VTE is not increased in long-haul commercial airline pilots, who are also exposed to prolonged periods of sitting (Kuipers et al., 2014) but it is possible that pilots with early presentation of VTE may have left this career for medical reasons and thus there may be selection bias in the sample examined.

It is informative to compare my findings of increased VTE risk prolonged work- and computer-related seated immobility and increasing mean hours seated in a 24-hour period with other studies investigating more general health outcomes associated with sedentary behaviour. Increasing sitting times at work have been associated with increased BMI in women (Eriksen et al., 2015), and increased sitting time for any activities is associated with increased risk of cardiovascular disease (Katzmarzyk, Church, Craig, \& Bouchard, 2009) and all-cause mortality (Katzmarzyk et al., 2009; Koster et al., 2012; Matthews et al., 2012; Alpa V. Patel et al., 2010) This association is independent of individual activity levels (Matthews et al., 2012; Alpa V. Patel et al., 2010). A dose-response effect between sitting and all-cause mortality has also been reported (Dunstan et al., 2010; Koster et al., 2012). All-cause mortality, must by definition include VTE events, however the low frequency of VTE as a fatal event, lack of routine autopsies and variable death certificate documentation would make it very difficult to identify the proportion of all-cause mortality rates that are directly attributable to VTE. Regardless, my findings are consistent with worse health outcomes associated with prolonged sitting. This is important, as increased occupational sitting times over the last decade have not been offset by higher levels of activity during leisure time (Jans et al., 2007), thus modifying the working environment is becoming an increasingly important aspect of reducing the deleterious effects of sedentary behaviour.

I assessed seated immobility only as it related to work and recreational computer use. I did not include immobility related to television watching, video-gaming, or tablet technology, 
nor did I include non-screen-based activities such as reading. A comprehensive retrospective study to assess all of these activities is likely to be difficult due to their variability and the uncertainty with recall. This is an important consideration when interpreting the findings of this study as I did not assess the potential role of these or other forms of seated immobility with VTE risk and cannot be sure that other immobility related behaviours were not more prevalent in one group than the other.

Travel represented the other risk factor related to prolonged seated immobility that was assessed in this study. I found a significantly increased risk of road travel $>4$ hours in the univariate, but not the multivariate analyses, and a significantly increased risk of international air travel in the multivariate but not the univariate analyses. Together with the observation that long distance road travel was about 2-fold more common than long distance air travel, these findings illustrate the importance of considering all forms of long distance travel, rather than just air travel in assessing VTE risk. This interpretation is consistent with the findings of the landmark case-control study of travel and VTE risk, in which travel $>4$ hours in the 4 weeks preceding presentation was associated with a 4 -fold increased risk of VTE, with about two-thirds of cases related to car travel (Ferrari et al., 1999). Similarly, the large population-based case-control study from the Netherlands reported that the VTE risk for flying was similar to the risks of travelling by car, bus or train, and the long distance car travel was the most common form of travel in this population (S. C. Cannegieter et al., 2006).

These findings of increased risk of VTE with seated immobility in a growing range of contexts, (occupational, recreational computer use, air travel, road travel), suggest that clinicians should consider seated immobility thrombosis in its widest context when assessing risk for VTE. This is all the more important when individuals may present with more than one context in which they are seated for prolonged periods of time.

The clinical relevance of my findings is suggested by the possibility that if protracted seated immobility is not identified as a possible risk factor for VTE, then VTE events may be classified as idiopathic. This could then lead to recommendations for indefinite, rather than 
time limited, anticoagulation with its concomitant risks, and failure to give lifestyle modification advice to reduce the risk of recurrence.

\section{Conclusions}

This study provides some evidence that prolonged sitting with work- and recreationalcomputer use is associated with VTE, particularly as mean hours seated in a 24 hour period increases. This type of immobility is unlikely to be recognised in clinical settings and could lead to misclassification of VTE as idiopathic, leading to prolonged, inappropriate anticoagulation and a missed opportunity to address modifiable risk factors. 


\section{Prolonged work- and computer-related immobility, a categorical or continuous variable?}

The West, Healy, and this case control study just described all showed an approximate $10 \%$ increased risk of VTE with each additional hour seated. West found that the maximum number of hours seated at work was associated with VTE, with the risk increasing by $10 \%$ per hour longer seated (odds ratio 1.1, 95\% $\mathrm{Cl} 1.0-1.2$ ) and that the maximum number of hours seated at work without getting up was associated with VTE, with the risk increasing by $20 \%$ per hour longer seated (odds ratio $1.2,95 \% \mathrm{Cl} 0.96-1.6$ ).

The Healy study showed that prolonged work- and computer related seated immobility (seated for at least 10 hours in a 24 hour period and at least two hours at a time without getting up) was associated with an increased risk of VTE, OR 2.8, (95\% Cl 1.2 to 6.1, $\mathrm{P}=0.013)$. The maximum and average number of hours seated in a 24 -hour period were also associated with an increased risk of VTE with an odds ratio of 1.1 (95\% $\mathrm{Cl} 1.0$ to 1.2 , $\mathrm{P}=0.008)$ and $1.1(95 \% \mathrm{Cl} 1.0$ to $1.2, \mathrm{P}=0.014)$ per additional hour seated respectively.

In my case-control study (Study One), maximum hours seated during a 24 hour period were associated with an increased risk of VTE of 1.08 (1.01 to 1.6, P=0.02) with each additional hour seated. However, the categorical variable of any episode of prolonged seated immobility was not significantly associated with an increased risk of VTE (OR 1.18, $95 \% \mathrm{Cl}$ 0.56 to $2.48, \mathrm{P}=0.67)$.

It is possible that in treating prolonged work- and computer-related immobility as a categorical variable there may have been information lost relative to a causal relationship between this behaviour and VTE. A more meaningful test may be to look for an association between the frequency of exposure to prolonged work- and computer-related seated immobility and VTE.

\section{An investigation of specific sedentary occupations}

In both the West case-control study and my own, there were a higher proportion of roles defined as sedentary (according to the ANZSCO criteria; Appendix IV) in the cases than in the controls. Point estimates of association were consistent with increased risk of VTE with all sedentary roles. However, the occupational roles are high level, with multiple sub-roles 
contributing to them, some of which might confer more risk than others. For example, one might hypothesise that IT workers such as computer programmers might be more at risk of prolonged occupational sitting and or prolonged computer related sitting at home compared to an IT technician who might have a more active role in installing or shifting computers or technical equipment. The Healy study collected more specific occupation codes and role descriptions from all cases and controls, as did the case-control study just presented, as it used a very similar methodology. In total, these two case-control studies collected detailed information on occupation and work- and computer-related immobility from a total of 396 individuals who had suffered a VTE and 397 controls who had experienced either a cardiovascular event requiring hospital admission (Healy, Levin, et al. 2010) or an upper limb injury requiring ongoing hospital outpatient management (I Braithwaite, Maher, et al., 2016).

This provided an opportunity to tease out whether there might be some more specific occupational classes that might confer an additional VTE risk compared to others. Combining the data of the two case-control studies also provides an opportunity to investigate the association between the frequency of exposure to seated immobility and VTE across all participants. The hypotheses under investigation included; that sedentary occupations are associated with a higher risk of VTE, that clerical and administrative workers and ICT professionals (a specific sub-group of 'Professionals') are independently associated with higher risk of VTE, and that increasing exposure to episodes of prolonged work- and computer-related sitting in the four weeks prior to the index event for the VE cases and controls is associated with VTE. 


\section{STUDY TWO: Seated immobility and the risk of venous \\ thromboembolism: a meta-analysis of two case-control studies.}

\section{Hypothesis}

The hypothesis of this study was that prolonged work- and computer-related immobility and sedentary occupations, particularly those of Administration and Clerical workers and Information, Communications and Technology (ICT workers) have an increased risk of VTE compared to those who do not have episodes of prolonged work- and computer-related immobility, non-sedentary workers and those who are not administrative and clerical or ICT workers.

\section{Primary Outcome variable}

The primary outcome variable was the odds ratio for association with VTE of prolonged work- and computer-related immobility (defined as being seated at work, and/or on the computer at home for at least 10 hours within a 24-hour period and, within that same period, for at least 2 hours without getting up at least once in the 28 day period prior to the index event for inclusion into the study). In this instance prolonged work- and computerrelated immobility was treated as a categorical variable.

\section{Secondary Outcome Variables}

Secondary outcome variables were; the odds ratio for association between sedentary occupations as categorised according to the Australia New Zealand Standard Classification of Occupations (ANZSCO) and VTE, the odds ratio for the association between administrative and clerical roles and VTE, the odds ratio for association between Information, Communications and Technology (ICT) and VTE and the odds ratio for association between the frequency of protracted work- and computer-related immobility as a continuous variable (i.e. the frequency that participants met the criteria for prolonged work- and computer-related immobility within in the 28 day period prior to the index event) and VTE. 


\section{Method}

This was an analysis of the combined data from Study One already reported (I Braithwaite et al. 2016), and the Healy case-control study published in 2010 (B. Healy, Levin, et al., 2010). Cases comprised 396 patients with radiologically confirmed deep vein thrombosis (DVT) or pulmonary embolism (PE) attending outpatient VTE clinics. Controls comprised 197 inpatients in the coronary care unit and 200 outpatients treated for upper limb injuries in the fracture clinic. Statistical analysis was by logistic regression analysis of combined data from two case-control studies which utilised similar methodology, to investigate the association of risk factors with VTE in adults aged 18 to 65 years, with a specific focus on prolonged work- and computer-related immobility, sedentary occupations and specific occupations of administrative and clerical workers and ICT workers. 


\section{Methods}

Data from the Healy study were collected between February 2007 and February 2009, comprising 196 VTE cases that had been treated at the Wellington Hospital VTE outpatient clinic and 197 controls that had been admitted to the Wellington Hospital CCU with non-VTE related problems. The Healy data were combined with the data collected for the casecontrol study just described, comprising 200 VTE cases seen at the Wellington Hospital VTE outpatient clinic and 200 controls who were outpatients with upper limb injuries managed through the Fracture Clinic outpatient system. Datasets in common included; age, sex, information about the index event, risk factors for VTE, job title and description of the role, ANZSCO code, average and maximum number of hours seated at work during the day and average and maximum number of hours seated at the computer for recreational computer use.

In the process of combining data, age, sex and information about the index event were all reviewed and confirmed against hospital records. Personal and family histories of VTE were confirmed by reviewing individual case report forms and referring to hospital records. Risk factors for VTE were compared between the two case-control studies to ensure consistent application of criteria, to attend to any inconsistencies in the way the data may have been gathered between the two studies, and to allow more detailed analysis of separate risk factors if possible.

Long distance travel was separated into travel by air and travel by other means of transport. Surgery for both studies was distinguished as either orthopaedic surgery or non-orthopaedic surgery, and analysis of both surgical categories was undertaken separately. Cast immobility was checked through hospital records the ensure accuracy of the account of the immobilisation used. Pregnancy and hormonal treatment were categorised separately to allow analysis of risk with pregnancy and the puerperium compared to the risk of OCP and hormone replacement therapy HRT use. In the Healy study, most chronic conditions had been assessed as probable VTE risk factors, thus $92 \%$ of the control group (CCU patients) were deemed to have medical risk factors for VTE. These conditions were reviewed, and 
only pertinent diagnoses were retained as risk factors, namely heart failure, renal failure, active cancers or their treatments and systemic inflammatory conditions.

In the original studies, all job roles for both case studies had been classified into each of nine high-level groups listed in the ANZSCO categorisation system; (1) Managers, (2) Professionals, (3) Technicians and Trades Workers, (4) Community and Personal Service Workers, (5) Clerical and Administrative Workers, (6) Sales Workers, (7) Machinery Operators and Drivers, (8) Labourers, and (9) Other. These were then classified into high sedentary (managers, professionals and clerical and administrative workers) and low sedentary categories (technicians and trade workers, community and personal service workers, sales workers, machinery operators and [some] drivers, labourers and not otherwise employed). All ANZSCO classifications were reviewed to ensure consistency between the two case studies. Using the information captured on the case report forms for both studies, all participants received a secondary sub-classification which identified more specific roles within the high level category. Administrative and Clerical workers as an overall category and Information and Communications Technology (ICT) workers as a subcategory of Professionals were examined separately for an association with VTE.

The criteria for prolonged work- and computer-related seated immobility remained unchanged between the studies (a minimum of 10 hours seated at work and/or at home with recreational computer use in a 24-hour period and a period of at least 2 hours without getting up). Participants had to meet these criteria at least once in the four weeks before the index event. If participants drove for their occupation (e.g. truck or taxi driver, courier), this was included in the immobility calculation, but commuting to and from work was not included, as commuting time had not been captured in my case-control study. Laptop use at home was not included, as this had not been captured in the Healy case-control study.

Prolonged work- and computer-related immobility was assessed both as a categorical and as a continuous variable. For the categorical variable, the odds ratio for association between VTE was calculated according to whether cases and controls had met the criteria for prolonged work- and computer-related immobility at all, 'yes' or 'no'. For the continuous variable, records from both case-control studies were reviewed to establish the frequency 
with which all participants met the criteria for prolonged work- and computer-related seated immobility within the four week period prior to their index event, and the total number of times each participant had met the criteria for prolonged-work and computer related immobility was calculated.

VTE risk factors for all cases and controls were grouped into categories as outlined in Table 2.5. 
Table 2.5: Risk factors for venous thromboembolism

\section{General Risk factors for VTE}

$1 \quad$ Age

2 Obesity $(\mathrm{BMI}>30 \mathrm{~kg} / \mathrm{m} 2)$

3 Sex

4 Personal History of VTE - confirmed previous VTE event $>6$ months prior to inclusion in this study

5 Family history of VTE - confirmed VTE in parents or siblings

6 Long distance travel in the four weeks prior to presentation

- Air travel: any international flights greater than 3.5 hours

- Road-based travel greater than 4 hours (car, bus, motorbike, camper-van)

7 Non-orthopaedic surgery requiring general anaesthetic or spinal / epidural anaesthesia in the four prior to the index event

8 Orthopaedic surgery in the 4 weeks prior to the index event

9 Cast immobility in the four weeks prior to index event

10 Case-control study one versus case-control study two

11 Pregnancy or hormonal treatment, including hormonal contraceptive, hormone replacement therapy, or hormonal antagonist therapy for malignancy

\section{Possible occupational risk factors for VTE}

12 Prolonged work- and computer-related seated immobility:

As a categorical variable - the duration seated at work and at home seated in front of a computer, in the four weeks prior to presentation, defined as: Seated for at least 10 hours in a 24 hour period and at least 2 hours at a time without getting up, at least once in the four weeks before presentation

As a continuous variable - the number of times a participant was exposed to prolonged workand computer-related seated immobility (as defined above) in the 28 days prior to the index event

13 Sedentary Occupation (Manager group, Professionals group, Administrative and Clerical Workers group)

14 Administrative and Clerical Workers group

15 ICT Professionals (sub-group of Administrative and Clerical Workers group)

BMI - Body mass index

VTE - Venous thromboembolism 


\section{Statistical Methods}

Univariate logistic regression was used to measure the strength of the association between case - control status and other explanatory variables. For the continuous variables the difference in mean values was also assessed with a simple t-test. In the multivariate logistic regression for the categorical variable of prolonged work- and computer-related seated immobility the predictor variables were: one or more episodes of prolonged work- and computer-related immobility in the 28 days prior to the index event, occupational class, sedentary occupation, administrative and clerical workers, and IT workers. These were adjusted for pre-specified confounding variables: Age, sex, BMI $>30 \mathrm{~kg} / \mathrm{m}^{2}$, personal history of VTE, family history of VTE, and any of the following within the 28 days prior to the development of symptoms of the index event; cast immobilisation, orthopaedic surgery, non-orthopaedic surgery, and long distance travel.

The multivariate regression model for the continuous variable of prolonged work- and computer-related seated immobility utilised the same predictor variables and pre-specified variables that were used in the categorical analysis.

\section{Power Calculation}

In the first case-control study, (B. Healy, Levin, et al., 2010) multivariate regression analysis showed that prolonged work- and computer-related seated immobility was associated with an increased risk of VTE, OR $2.8,(95 \% \mathrm{Cl} 1.2$ to $6.1, \mathrm{P}=0.013)$. In this combined analysis, a sample size of 800 (400 cases and 400 controls from both case-control studies combined), would give $90 \%$ power with a type one error rate of $5 \%$ to detect an odds ratio for risk of VTE of 2.8 if the case rate was as low as $10 \%$ and the control rate as low as $4 \%$. 


\section{Results}

In total I reviewed 396 cases and 397 controls. Characteristics of all participants are shown in Table 2.6. The mean age of all cases was 45.9 years and 47.11 years in the controls. At least one episode of prolonged work- and computer-related seated immobility in the 4 weeks prior to their index event was reported in 63 cases and 48 controls. The proportion of obese participants in both groups was similar. Females comprised almost half of the cases and just over one third of the controls, reflecting the Healy study where the majority of the CCU controls were male. The number of cases claiming a personal history of VTE outnumbered controls ten to one and there were twice as many cases with a family history of VTE compared to the controls. 57 cases had undertaken long distance travel of any sort in the four weeks prior to their index event compared to 20 controls. $7.6 \%$ and $9.6 \%$ of the cases had undergone non-orthopaedic and orthopaedic surgery respectively compared to $1.3 \%$ and $0.3 \%$ of the controls. 62 of the cases had undergone cast immobility compared to 2 of the controls.

Of the cases, 235 worked in sedentary roles, of which 54 were administrative and clerical workers and 30 were ICT professionals, while in the controls 181 worked in sedentary roles, 39 were administrative and clerical workers and 17 were ICT professionals.

The mean (SD) number of days that participants reported prolonged work- and computerrelated seated immobility in the 28 days prior to their index event was 1.18 (3.78) in the cases and 1.03 (3.67) for the controls. The mean (SD) number of days that all sedentary occupations, clerical and administrative workers and ICT professionals reported prolonged work- and computer-related immobility was 1.62 (4.39), 0.57 (1.72) and 2.87 (5.51) for the cases and 1.51 (4.26), $0.18(0.8)$ and 1.94 (4.62) for the controls respectively (Table 2.6). 
Table 2.6: Characteristics of study participants

\begin{tabular}{lll}
\hline & Mean (SD) & \\
\hline Age (years) & $45.9(12.4)$ & $47.1(11.8)$ \\
& $\mathrm{N}=396$ & $\mathrm{~N}=395$ \\
\hline Case N/N (\%) & Control N/N (\%) \\
\hline BMI >30 & $151 / 390(38.7)$ & $137 / 395(34.7)$ \\
Female sex & $189 / 396(47.7)$ & $145 / 397(36.5)$ \\
Personal history VTE & $69 / 396(17.4)$ & $7 / 397(1.8)$ \\
Family history VTE & $82 / 396(20.7)$ & $41 / 397(10.3)$ \\
Long distance travel & $57 / 396(14.4)$ & $20 / 397(5.0)$ \\
Non-orthopaedic surgery & $30 / 396(7.6)$ & $5 / 397(1.3)$ \\
Orthopaedic surgery & $38 / 396(9.6)$ & $1 / 397(0.3)$ \\
Cast Immobility & $62 / 396(15.7)$ & $2 / 397(0.5)$ \\
First case-control-study versus second case-control & $196 / 396(49.5)$ & $197 / 397(49.6)$ \\
study & & \\
\hline
\end{tabular}

\section{Proposed occupational risk factors for VTE}

\begin{tabular}{lll}
\hline $\begin{array}{l}\text { At least one episode of prolonged work- and } \\
\text { computer-related seated immobility in the } 28 \text { days } \\
\text { prior to the index event }\end{array}$ & $63 / 396(15.9)$ & $48 / 397(12.1)$ \\
& & \\
Sedentary occupation & $235 / 396(59.3)$ & $181 / 397(45.7)$ \\
Administrative and Clerical Workers & $54 / 396(13.6)$ & $39 / 397(9.8)$ \\
ICT Professionals & $30 / 396(7.6)$ & $17 / 397(4.3)$
\end{tabular}

Mean number of exposures to prolonged work- and computer-related seated immobility in the 28 day period prior to the index event

\begin{tabular}{lll}
\hline All cases (N=396) and controls ( $\mathrm{N}=397)$ & $1.18(3.78)$ & $1.03(3.67)$ \\
Sedentary occupations & $1.62(4.39)$ & $1.51(4.26)$ \\
Administrative and clerical workers & $0.57(1.72)$ & $0.18(0.8)$ \\
ICT professionals & $2.87(5.51)$ & $1.94(4.62)$ \\
& & \\
\hline SD - Standard Deviation & & \\
BMI - Body Mass Index & & \\
VTE - Venous thromboembolism & & \\
ICT - Information, Communication and Technology &
\end{tabular}


The number(\%) of cases meeting the criteria for at least one episode of prolonged workand computer-related immobility was $51(22 \%), 8(15 \%)$ and $10(33 \%)$ in sedentary occupations, administrative and clerical workers and ICT professionals respectively (Table 2.7). The number(\%) of controls meeting the criteria for at least one episode of prolonged work- and computer-related immobility was 34(19\%), 2(5\%) and 3(18\%) in sedentary occupations, administrative and clerical workers and ICT professionals respectively. 
Table 2.7: Study participants by occupational class.

\begin{tabular}{|c|c|c|c|c|}
\hline & \multicolumn{2}{|c|}{$\begin{array}{l}\text { Cases } \\
\mathrm{N}=396\end{array}$} & \multicolumn{2}{|c|}{$\begin{array}{l}\text { Controls } \\
\mathrm{N}=397\end{array}$} \\
\hline Occupational Group & $\begin{array}{l}\text { Number in } \\
\text { Occupational } \\
\text { Group or sub- } \\
\text { group: N(\%) }\end{array}$ & $\begin{array}{l}\text { Meet criteria for } \\
\text { prolonged work- } \\
\text { and computer- } \\
\text { related seated } \\
\text { immobility: } \mathrm{N}(\%)\end{array}$ & $\begin{array}{l}\text { Number in } \\
\text { Occupational } \\
\text { Group or sub- } \\
\text { group: N(\%) }\end{array}$ & $\begin{array}{l}\text { Meet criteria for } \\
\text { prolonged work- } \\
\text { and computer- } \\
\text { related seated } \\
\text { immobility: } \mathrm{N}(\%)\end{array}$ \\
\hline \multicolumn{5}{|l|}{ Sedentary Occupations } \\
\hline TOTAL SEDENTARY & $235(59.6)$ & 51 (21.6) & $181(45.6)$ & $34(18.8)$ \\
\hline Managers & 47 (11.9) & $10(20.8)$ & $47(11.8)$ & $11(23.4)$ \\
\hline Professionals & $134(33.8)$ & $33(24.6)$ & 95 (23.9) & $21(22.1)$ \\
\hline $\begin{array}{l}\text { Clerical and administrative } \\
\text { workers }\end{array}$ & $54(13.6)$ & $8(14.8)$ & $39(9.8)$ & $2(5.1)$ \\
\hline $\begin{array}{l}\text { SUBGROUP: } \\
\text { ICT Professionals }\end{array}$ & $30(7.6)$ & $10(33.3)$ & $17(4.3)$ & $3(17.6)$ \\
\hline \multicolumn{5}{|l|}{ Non-sedentary Occupations } \\
\hline TOTAL NON-SEDENTARY & $161(40.0)$ & $12(7.5)$ & $216(54.4)$ & $14(6.5)$ \\
\hline $\begin{array}{l}\text { Technicians and trade } \\
\text { workers }\end{array}$ & $22(5.5)$ & $3(13.6)$ & $48(12.1)$ & $0(0.0)$ \\
\hline $\begin{array}{l}\text { Community and personal } \\
\text { service workers }\end{array}$ & $20(5.1)$ & $2(10.0)$ & $23(5.8)$ & $2(8.7)$ \\
\hline Sales workers & $17(4.3)$ & $1(5.9)$ & $22(5.5)$ & $0(0.0)$ \\
\hline $\begin{array}{l}\text { Machinery operators and } \\
\text { drivers }\end{array}$ & $9(2.3)$ & $1(11.1)$ & $18(4.5)$ & $2(11.1)$ \\
\hline Labourers & $12(3.0)$ & $0(0.0)$ & $28(7.1)$ & $1(3.6)$ \\
\hline $\begin{array}{l}\text { Not in paid employment } \\
\text { and not in voluntary role }\end{array}$ & $79(19.9)$ & $4(5.1)$ & $76(19.1)$ & $9(11.8)$ \\
\hline Unable to be classified & $2(0.5)$ & $1(50.0)$ & $1(0.3)$ & $0(0.0)$ \\
\hline
\end{tabular}

Prolonged work- and computer-related seated immobility - seated for a minimum of 10 hours in a 24 hour period, with a minimum period of two hours without getting up.

ICT - Information, Communication and Technology - a sub-group of Professionals 


\section{Prolonged work- and computer-related seated immobility and VTE}

Table 2.8 shows the univariate and multivariate associations of risk factors for VTE when considering risk factors for VTE as well as prolonged work- and computer-related seated immobility as a categorical variable. As expected, increasing age was significantly associated with VTE in both the univariate and multivariate analysis, as was a personal and family history of VTE. Long distance travel, non-orthopaedic and orthopaedic surgery were all associated with VTE in the multivariate analysis. BMI was not associated with increased risk of VTE.

There was no association between prolonged work- and computer-related seated immobility and VTE, although the point estimates (1.39 in univariate analysis and 1.24 in multivariate analysis) were consistent with increased risk of VTE.

There was a significant association between sedentary occupations and VTE with an OR for association of $1.79(95 \% \mathrm{Cl} 1.22$ to $2.63, \mathrm{P}=0.003)$.

There was no association between administrative and clerical roles and VTE in either the univariate or multivariate analysis.

ICT roles were associated with VTE in univariate analysis; OR 1.85 (1.00 to 3.41, $\mathrm{P}=0.049$ ), but in multivariate regression the association was not significant; OR 1.13 (95\% Cl 0.53 to $2.41, \mathrm{P}=0.75)$, although again, the point estimates were consistent with increased risk. 
Table 2.8: Odds Ratios $(95 \% \mathrm{CI}) *$ for association of risk factors and VTE with prolonged work- and computer-related seated immobility as a categorical variable.

\begin{tabular}{|c|c|c|}
\hline & $\begin{array}{l}\text { Odds ratio }(95 \% \mathrm{Cl}) \\
\mathrm{P} \text { value } \\
\text { Univariate }\end{array}$ & Multivariate \\
\hline \multirow[t]{2}{*}{ Age per decade older } & $0.93(0.82$ to 1.04$)$ & 0.83 (0.72 to 0.96$)$ \\
\hline & 0.19 & 0.014 \\
\hline \multirow[t]{2}{*}{$\mathrm{BMI}>30 \mathrm{Kg} / \mathrm{m}^{2}$} & 1.19 (0.89 to 1.59$)$ & 1.14 (0.80 to 1.63$)$ \\
\hline & 0.25 & 0.47 \\
\hline \multirow[t]{2}{*}{ Female sex } & 1.57 (1.18 to 2.09$)$ & 1.50 (1.04 to 2.14$)$ \\
\hline & 0.002 & 0.028 \\
\hline \multirow[t]{2}{*}{ Personal history VTE } & 11.7 (5.29 to 25.8$)$ & 17.4 (7.59 to 39.8$)$ \\
\hline & $<0.001$ & $<0.001$ \\
\hline \multirow[t]{2}{*}{ Family history VTE } & 2.36 (1.57 to 3.54$)$ & 1.80 (1.11 to 2.93$)$ \\
\hline & $<0.001$ & 0.017 \\
\hline \multirow[t]{2}{*}{ Long distance travel } & 3.14 (1.84 to 5.33$)$ & 4.11 (2.29 to 7.38$)$ \\
\hline & $<0.001$ & $<0.001$ \\
\hline \multirow[t]{2}{*}{ Non-orthopaedic surgery } & $6.48(2.49$ to 16.9$)$ & $8.53(3.12$ to 23.3$)$ \\
\hline & $<0.001$ & $<0.001$ \\
\hline \multirow[t]{2}{*}{ Orthopaedic surgery } & 42.4 (5.80 to 310.4$)$ & 59.6 (7.79 to 455.7$)$ \\
\hline & $<0.001$ & $<0.001$ \\
\hline \multirow[t]{2}{*}{ Cast immobility } & 36.3 (8.8 to 149.7$)$ & 41.9 (10.0 to 176.1$)$ \\
\hline & $<0.001$ & $<0.001$ \\
\hline First case-control study versus second & $0.98(0.74$ to 1.30$)$ & 1.02 (0.73 to 1.43$)$ \\
\hline case-control study & 0.88 & 0.91 \\
\hline \multicolumn{3}{|l|}{ Occupational Risk Factors } \\
\hline \multirow{2}{*}{$\begin{array}{l}\text { Prolonged work- and computer- } \\
\text { related seated immobility }\end{array}$} & 1.39 (0.93 to 2.08$)$ & 1.24 (0.75 to 2.03 ) \\
\hline & 0.11 & 0.40 \\
\hline \multirow[t]{2}{*}{ Sedentary occupation } & 1.75 (1.32 to 2.32$)$ & 1.79 (1.22 to 2.63$)$ \\
\hline & $<0.001$ & 0.003 \\
\hline \multirow[t]{2}{*}{ Administrative and Clerical Workers } & 1.43 (0.92 to 2.22$)$ & 1.08 (0.61 to 1.90$)$ \\
\hline & 0.11 & 0.79 \\
\hline \multirow[t]{2}{*}{ ICT professionals } & 1.85 (1.00 to 3.41$)$ & 1.13 (0.53 to 2.41$)$ \\
\hline & 0.049 & 0.75 \\
\hline \multicolumn{3}{|l|}{$\begin{array}{l}\text { *All adjusted for Age, BMI, personal history of VTE, } \\
\text { non-orthopaedic surgery, cast immobility and occup } \\
\text { CI - confidence interval } \\
\text { VTE - venous thromboembolism } \\
\text { BMI - body mass index } \\
\text { ICT - information and communications technology }\end{array}$} \\
\hline
\end{tabular}


The associations of risk factors for VTE when considering exposure to prolonged work- and computer-related seated immobility as a continuous variable (mean number of exposures to prolonged seated immobility in the four weeks prior to the index event) are shown in Table 2.9. Although the point estimates were slightly different from the first multivariate analysis in Table 2.8 with prolonged work- and computer-related seated immobility as a categorical variable, the significant associations between increasing age, sex, personal and family history of VTE, long distance travel, surgery; both non-orthopaedic and orthopaedic, and cast immobility remained.

The point estimates for prolonged work and computer related immobility in both univariate and multivariate analysis were consistent with a higher risk of VTE, but the associations were not significant.

There was a significant association between sedentary occupations and VTE in both the univariate (OR 1.75, $\mathrm{Cl} 1.32$ to $2.32, \mathrm{P}<0.001$ ) and multivariate analysis (OR 1.85, $\mathrm{Cl} 1.26$ to 2.71, $P=0.002)$.

ICT professionals were associated with an increased risk of VTE in the univariate analysis, but this was not significant in the multivariate analysis.

While there was a suggestion of an association between VTE risk and being an administrative and clerical worker in the univariate analysis, this also did not bear out in the multivariate analysis. 
Table 2.9: Odds Ratios $(95 \% \mathrm{Cl}) *$ for association of risk factors and VTE with frequency of prolonged work- and computer-related seated immobility as a continuous variable.

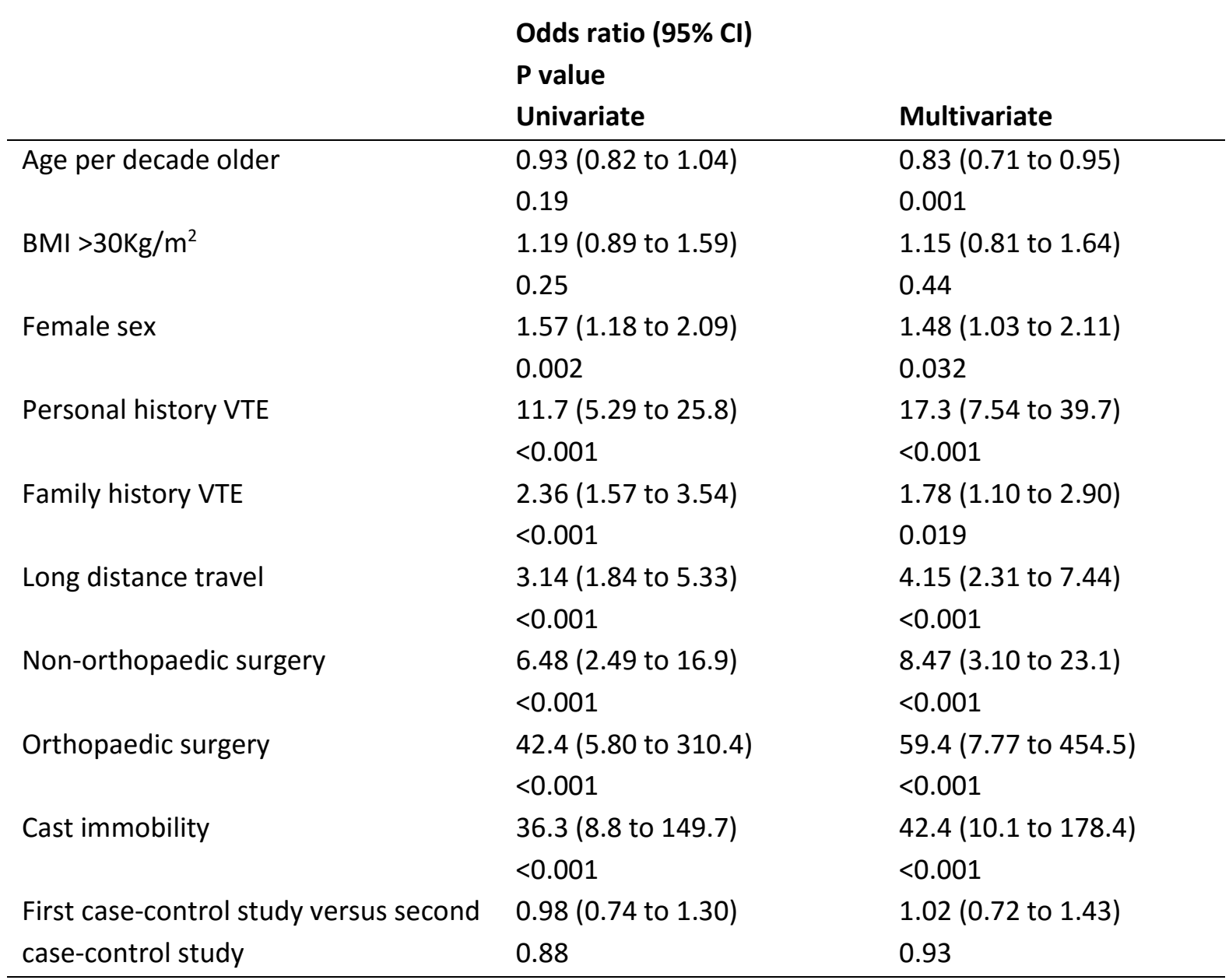

Occupational Risk Factors

\begin{tabular}{|c|c|c|}
\hline Prolonged work- and computer- & 1.01 (0.97 to 1.05$)$ & $1.00(0.96$ to 1.05$)$ \\
\hline related seated immobility & 0.56 & 0.91 \\
\hline \multirow[t]{2}{*}{ Sedentary occupation } & 1.75 (1.32 to 2.32$)$ & 1.85 (1.26 to 2.71$)$ \\
\hline & $<0.001$ & 0.002 \\
\hline \multirow[t]{2}{*}{ Administrative and Clerical Workers } & 1.43 (0.92 to 2.22$)$ & 1.06 (0.60 to 1.86$)$ \\
\hline & 0.11 & 0.85 \\
\hline \multirow[t]{2}{*}{ ICT professionals } & 1.85 (1.00 to 3.41$)$ & 1.13 (0.53 to 2.41$)$ \\
\hline & 0.049 & 0.75 \\
\hline
\end{tabular}




\section{Discussion}

Sedentary occupations were associated with an increased risk of VTE with an OR of 1.76 (95\% $\mathrm{Cl} 1.2$ to $2.6, \mathrm{P}=0.004)$. There was no association between prolonged work- and computer-related seated immobility and VTE when considered as a categorical variable; OR $1.24(95 \% \mathrm{Cl} 0.75$ to $2.03, \mathrm{P}=0.4)$, nor was there an association between increasing frequency of exposure to prolonged work- and computer-related seated immobility and VTE; OR 1.00 (95\% $\mathrm{Cl} 0.96$ to $1.05, \mathrm{P}=0.91$ ). ICT professionals had increased risk of association for VTE in the univariate analysis, and a point estimate consistent with increased risk of association in the multivariate analyses that was not statistically significant. There was no evidence of increased risk of association for VTE in the clerical and administrative workers group in either univariate or multivariate analyses.

The finding of an association between sedentary occupations and VTE risk is consistent with studies that have shown increased risk of VTE with increasing duration of travel (Lapostolle et al., 2001; Perez-Rodriguez et al., 2003), and with fewer episodes of mobilising while travelling (Paganin et al., 2003). In both the case-control studies contributing to this current analysis (B. Healy, Levin, et al., 2010) outlined in the beginning of this chapter, increasing mean hours seated in a 24-hour period; a characteristic that would be expected in sedentary occupations; is significantly associated with VTE. Unfortunately due to differences in the way the data were collected between the two studies, I was unable undertake a combined analysis of that variable and so cannot confirm this historical finding.

Of broader public health interest, the increased VTE risk in sedentary occupations is broadly consistent with scholarship investigating sedentary behaviour and other health outcomes. Not only have increasing sitting times at work been associated with increased BMI (Brown, Miller, \& Miller, 2003; Clark et al., 2009; Eriksen et al., 2015), but increased sitting time in any capacity throughout the day is associated with an increased risk of idiopathic PE in women (Kabrhel et al., 2011), an increased risk of cancers (Schmid \& Leitzmann, 2014), and an increased risk of cardiovascular disease and all-cause mortality (Jakes et al., 2003; Katzmarzyk et al., 2009; Koster et al., 2012; Matthews et al., 2012; Alpa V. Patel et al., 2010). A dose-response effect between sitting and all-cause mortality has also been reported with 
an $11 \%$ higher risk of mortality with every one hour increase in television watching (Dunstan et al., 2010) and all-cause mortality hazard ratios increasing with increasing sedentary time (van der Ploeg, Chey, Korda, Banks, \& Bauman, 2012).

Not only is increased sitting associated with poor health outcomes, but this is independent of individual activity levels, with Chau and colleagues estimating a $34 \%$ higher risk of allcause mortality in adults sitting for ten or more hours per day independent of physical activity levels (Chau et al., 2013), Patel and colleagues reporting higher cancer and mortality rates with prolonged sitting independent of activity levels (Alpa V. Patel et al., 2010) and Koster and colleagues reporting higher mortality rates with increasing sedentary time independent of any levels of moderate to vigorous physical activity (Koster et al., 2012). Of concern, despite an increase in occupational sitting times over the last decade, this has not been offset by higher levels of activity during leisure time (Jans et al., 2007), thus modifying the working environment to reduce sedentary behaviour is becoming a more important aspect of reducing the associated deleterious health effects of prolonged sitting.

Prolonged work- and computer-related seated immobility as a categorical variable had a point estimate consistent with increased risk of VTE in the univariate analysis, but this was not statistically significant. After multivariate regression analysis, which included other proposed occupational risk factors, prolonged work- and computer-related seated immobility had no risk of association with VTE. If the episodes of seated immobility were associated with sedentary occupations of the participants, inclusion of sedentary occupations in the multivariate regression analysis might explain the lack of association found between prolonged work- and computer-related immobility and VTE in the multivariate analysis.

Anticipating that treating prolonged work- and computer-related seated immobility as a categorical variable might lose information relevant to a causal relationship between this kind of immobility and VTE, I tested for an association between the frequency with which participants were exposed to prolonged work- and computer-related seated immobility in the 28 days prior to their index event and VTE. There was no evidence of an association between frequency of exposure to prolonged work- and computer-related seated 
immobility and risk for VTE in univariate or multivariate analysis. This could be because the study was underpowered to detect such an association, or possibly because it is the overall sedentary nature of the role that is associated with VTE rather than the frequency with which a measure of sedentary work is exceeded.

I hypothesised that within sedentary occupations, ICT professionals (a sub-group of Professionals) and clerical and administrative workers would be independent risk factors for VTE. I chose these groups specifically, because they are most likely to represent patients previously described in case reports of VTE after extended periods of sitting and gaming, they were more likely to have a significant seated component in their role and because there would be a sufficient number of cases and controls in each group to test for an association. ICT professionals had a point estimate consistent with an increased risk of VTE in univariate but not multivariate regression, while there was no evidence for increased risk of VTE in the clerical and administrative workers group. It is likely that I did not have enough power in this combined analysis to detect a difference in risk for VTE in these occupations. It is also likely that it is the sedentary nature of the role that carries the real risk of association with VTE. This is highlighted by a point estimate of increased risk of VTE in the univariate analysis of ICT professionals, which was not significant with the inclusion of all sedentary roles in the multivariate regression analysis. It may be that focussing on increasing physical activities in all sedentary occupations rather than specific work groups would be of greater public health benefit.

There are a number of methodological issues to take into account with this analysis. The allocation of occupational class to each participant was on the basis of stated job titles and very brief role descriptions. It is possible that the occupational class does not fully or accurately reflect the actual role or activity levels of each individual allocated to that class. This is inherent in any classification system and occupational classes were reviewed and applied consistently across all cases and all controls from both studies in an attempt reduce bias.

Immobility was assessed on retrospective participant recall of average sedentary behaviours in their workplace, which is always prone to recall bias in these kinds of studies. A 
prospective study might have taken advantage of more objective measures of sedentary / active behaviours, such as accelerometers or seat pressure monitors. In this combined analysis, immobility was assessed only as it related to work and recreational computer use. Commuting time to and from work and time spent on laptops either at home or for work related activities was not able to be included as they were collected in one case-control study only. Data on reading, television watching, video gaming or other screen-time were not collected, thus the full duration of sedentary time during a single day was not captured. Capturing detailed information relating to all these activities would be problematic in a retrospective case-control study, and it is unclear how the addition of other sedentary measures might influence the results of this analysis.

Regardless, it is useful for both employers and employees to understand how sedentary occupations may contribute to the risk of VTE and other comorbidities and to develop strategies to decrease sitting time in the workplace. For health professionals, prolonged work- and computer-related seated immobility is not a readily recognised risk factor for VTE in clinical settings, and failure to recognise this risk could lead to the diagnosis of an idiopathic VTE. This could lead to prolonged inappropriate anticoagulation and a missed opportunity to address modifiable risk factors.

\section{Conclusions}

This study has shown that sedentary occupations are associated with an increased risk of VTE. This is consistent with previous studies on long distance road and air travel, and studies that have shown increased morbidity and mortality with increased sitting times independent of exercise. There may be value in employers assessing their workplace environment and considering interventions to reduce protracted sitting times associated with the roles of their employees. More accurate assessment of sedentary behaviours in the workplace could be achieved with the use of accelerometers or seat sensors in prospective studies of workplace sitting behaviours. 


\section{Chapter Three: Lower limb immobilisation and the risk of venous thromboembolism}

\section{Background}

In 1934, Mr John Homans who we have already come across in the medical literature due to his discourse on immobility and VTE, reported a 'dangerous form of venous thrombosis uncommon enough to make its study difficult, yet so often fatal as to make its recognition a matter of importance...' (Homans, 1934). He went on to describe the local signs and symptoms of DVT of the leg - recurring calf discomfort, ankle swelling and venous discoloration which might resolve with bed rest but would soon recur with obtaining the upright position. On autopsy after fatal PEs in a series of cases he described, large thrombi were found in the large veins of the calf muscles, with the femoral vein unobstructed, but long large clots had apparently broken off and lodged in the pulmonary vasculature of the lungs.

Homans proffered the suggestion that this kind of thrombus behaved differently from the more common types of thrombosis known at the time; thrombophlebitis of varicose veins (a problem associated with the vein wall), phlebitis migrans (a localised inflammatory lesion), and phlegmasia alba dolens (leg swelling associated with pelvic thrombi and involving solid obstruction of the great veins). He hypothesised that with growth over time, and with exertion, small clots that may be harboured for months within the network of veins of the calf would propogate upwards into larger veins, and when they met an unobstructed vein with a 'strong current' the proximal end of the clot would dislodge, and cause a PE. He described two particular cases in further depth;

- A 50 year old man with a metatarsal fracture who had his lower leg placed within a cast for one week, and then was limited to crutches. He suffered leg swelling and pain whenever he walked. He remained on crutches for some time and died from a PE four months later. On autopsy, the femoral vein was not occluded and one of the popliteal veins contained a thrombus that had a clean fractured surface from which 
it was surmised the PE had broken off. The popliteal thrombus extended into the veins of the soleus muscle that also appeared organised.

- An active 45 year old man 'rather plethoric and stout' who developed a per-patellar bursitis not long after suffering a severe cold. He spent almost two weeks in bed, and then on rising developed a swollen cyanotic leg and 'oppressiveness' in his chest, with breathlessness. After 2 days bedrest, the leg symptoms were resolved but he had fever most afternoons. He was mobilising on crutches most of the time and bearing no weight on his affected leg. One week later he died from PE. In this instance autopsy revealed a long organised clot in his lungs, extensive thrombosis of the muscular veins of both calf and thigh, and a thrombus in the popliteal vein that appeared to have broken at the proximal end as it was 'waving free in the femoral vein'. Of the three clots found in his calf veins, one was deemed likely to have been there for months.

Homan's conclusion was that this phenomenon was poorly recognised, and death might be prevented by the ligation of the femoral vein to prevent any embolism from travelling to the lungs. The cases described might be the first consolidation of Virchows' description of distal DVTs breaking off and forming PE in the context of lower limb injury and immobilisation. Since this time a number of similar case reports have followed (Venkatachalam \& Wright 2012)

The association between lower limb trauma, from minor injuries to fractures with or without consequent immobilisation, and VTE have since been demonstrated (J. Heit et al., 2000; Huerta et al., 2007; Samama, 2011; Mark Testroote et al., 2014). However, the practice of routine anticoagulation in the setting of lower limb immobilisation is varied due to lack of agreement on the natural history of isolated distal DVTs, the clinical significance of asymptomatic distal DVTs involving one or more of the deep calf veins without extension into the popliteal vein, and the variable incidence of DVT found across the broad spectrum of lower limb trauma and immobilisation. 


\section{Natural history of isolated distal deep vein thrombosis}

An isolated distal deep vein thrombosis (IDDVT) involves one or more of the deep calf veins without extension into the popliteal vein. The natural history of an asymptomatic IDDVT is still unclear, as generally, once discovered, they are treated due to concerns about possible extension into proximal veins. In small number of symptomatic IDDVT patients followed for one year, $23 \%$ of patients had persisting symptoms and $24 \%$ developed valvular incompetence within one year (Meissner, Caps, Bergelin, Manzo, \& Strandness, 1997). Whether the patients were anticoagulated is not reported.

Approximately $14 \%$ of high risk trauma patients, already on prophylaxis will develop IDDVT of which $5 \%$ will propagate proximally requiring a change in management (Sharpe et al., 2002). In a small pilot study of therapeutic warfarin versus conservative management in patients diagnosed with IDDVT, $11 \%$ of the conservatively managed group suffered either propagation into the popliteal vein, PE or VTE-related death (Horner, Hogg, Body, Nash, \& Mackway-Jones, 2012). These studies are small, but would indicate that somewhere between 5 and $10 \%$ of patients with symptomatic, diagnosed IDDVT will go on to develop VTE events that require treatment. The prevalence of asymptomatic IDDVT in the general population is not known, and the natural history of these cannot be inferred. 


\section{The medical literature: Prevalence of venous thromboembolism in lower limb trauma or immobilisation}

In the medical literature, the incidence of IDDVT and / or proximal DVT has varied widely due to different criteria for diagnosis between studies. Some studies have utilised radiological screening and others have reported retrospectively from hospital records on symptomatic and then subsequently radiologically confirmed events. Even with radiologically confirmed diagnoses of symptomatic events, reported prevalence of IDDVT in patient groups varies greatly depending on the diagnostic method employed; venography or ultrasound (US), and whether serial USs of the proximal veins are undertaken or a single full US examination of all the of accessible veins of the lower limb (Palareti \& Sartori, 2016).

Patient groups in the medical literature are widely varied, at times are poorly defined, may have received varying degrees of chemoprophylaxis for VTE, and may have variable exclusion or inclusion of higher risk patients. The groups may also comprise patients with any of fractures of the long bones of the leg and / or the foot (potentially also including other trauma), soft tissue injuries anywhere below the knee, elective or post-traumatic operative interventions from the knee down and immobilisation either in plaster casts, fibreglass casts, moonboots or bandages, and at times post-treatment immobilisation and weight-bearing capacity has not been described. Hence accurate quantification of VTE events in specific patients groups is difficult and not surprisingly, there is little consensus amongst clinicians with respect to prophylaxis for VTE in this clinical setting.

As can be seen in the following tables, the estimated risk of VTE varies greatly according to the design of the study and the patient group examined. In the case of data repository reviews (Table 3.1), the reported VTE rates in foot and ankle surgery are low. However, the investigators are usually unable to ascertain whether anticoagulation has been given routinely, or at least to high risk patients in the dataset and in one instance, an estimation of DVT rates was not attempted (SooHoo et al., 2009). In this broad patient group, where it is possible higher risk patients were treated differently, the rates of symptomatic VTE were between 0.4 and $6.3 \%$. 
In the placebo arms of randomised controlled trials undertaken prior to 2010, where VTE events have been identified through screening, reported VTE events have ranged from 4.3 to $36 \%$ (Table 3.2). Again, the patient groups are mixed, some surgical, some conservatively managed, immobilisation techniques have not all been clearly identified, some received VTE prophylaxis and some did not, and in the main, only low risk patients were recruited. Also, in the studies that specified the location of diagnosed DVTs, the majority were IDDVTs, of which 5 to $10 \%$ might be expected to propagate proximally. Of note, the final study in this list, published in 2015 had a comparatively low VTE rate, but it should be noted that chemoprophylaxis was given until all patients were independently mobile, therefore patients were weight-bearing before prophylaxis was stopped, and only proximal VTEs were screened for and reported (Selby et al., 2015).

Table 3.3 lists a range of prospective cohort studies that used a mix of records review and active screening. VTE rates again ranged from $0.4 \%$ to $7.0 \%$. Most events were DVTs but unfortunately they are not all consistently classified into proximal and distal events. 
Table 3.1: Retrospective reviews of data for VTE events in patient groups

\begin{tabular}{|c|c|c|c|c|c|c|c|c|}
\hline Study author & Type of study & Patient group & $\begin{array}{l}\text { Type of lower } \\
\text { limb } \\
\text { immobilisation }\end{array}$ & Thromboprophylaxis? & $\begin{array}{l}\text { Symptomatic / } \\
\text { asymptomatic? }\end{array}$ & DVT rate & PE rate & $\begin{array}{l}\text { Overall } \\
\text { VTE rate }\end{array}$ \\
\hline $\begin{array}{l}\text { Wukich } 2008 \\
\text { (Wukich \& } \\
\text { Waters, 2008) }\end{array}$ & $\begin{array}{l}\text { Retrospective } \\
\text { review }\end{array}$ & $\begin{array}{l}1000 \text { elective } \\
\text { foot operation } \\
\text { patients }\end{array}$ & Varied & $\begin{array}{l}\text { Proportion received } \\
\text { prophylaxis if hospital } \\
\text { stay involved }\end{array}$ & Symptomatic & $0.4 \%$ & $0.3 \%$ & $0.7 \%$ \\
\hline $\begin{array}{l}\text { SooHoo } 2009 \\
\text { (SooHoo et } \\
\text { al., 2009) }\end{array}$ & $\begin{array}{l}\text { Data from } \\
\text { California } \\
\text { discharge } \\
\text { database }\end{array}$ & $\begin{array}{l}\text { ORIF of ankle } \\
\text { fracture between } \\
1995 \text { and } 2005 \\
(\mathrm{~N}=57,183)\end{array}$ & Unclear & Unknown & Symptomatic & $\begin{array}{l}\text { Not } \\
\text { attempted }\end{array}$ & $0.34 \%$ & \\
\hline $\begin{array}{l}\text { Jameson } 2011 \\
\text { (Jameson et } \\
\text { al., 2011) }\end{array}$ & $\begin{array}{l}\text { Dataset } \\
\text { analysis }\end{array}$ & $\begin{array}{l}\text { Ankle fracture } \\
(\mathrm{N}=45,949) \text { or } \\
\text { replacement } \\
(\mathrm{N}=1,633) \text { first } \\
\text { metatarsal } \\
\text { osteotomy } \\
(\mathrm{N}=33,626) \text {, } \\
\text { hindfoot fusions } \\
(\mathrm{N}=7,033)\end{array}$ & Not described & Not ascertained & Symptomatic & $\begin{array}{l}\text { Ankle } \\
\text { fracture } \\
0.12 \%\end{array}$ & $\begin{array}{l}\text { Ankle } \\
\text { fracture } \\
0.17 \%\end{array}$ & $0.3 \%$ \\
\hline $\begin{array}{l}\text { Patel 2012(A } \\
\text { Patel, Ogawa, } \\
\text { Charlton, \& } \\
\text { Thordarson, } \\
\text { 2012) }\end{array}$ & $\begin{array}{l}\text { Retrospective } \\
\text { review of } \\
\text { database }\end{array}$ & $\begin{array}{l}\text { Achilles tendon } \\
\text { ruptures } \\
(\mathrm{N}=1172)\end{array}$ & $\begin{array}{l}\text { Plantarflexed } \\
\text { immobilisation }\end{array}$ & 'Not routinely' & Symptomatic & $0.43 \%$ & $0.34 \%$ & $0.77 \%$ \\
\hline
\end{tabular}




\begin{tabular}{|c|c|c|c|c|c|c|c|c|}
\hline $\begin{array}{l}\text { Shibuya2012 } \\
\text { (Shibuya, } \\
\text { Frost, } \\
\text { Campbell, } \\
\text { Davis, \& } \\
\text { Jupiter, 2012) }\end{array}$ & $\begin{array}{l}\text { National } \\
\text { trauma } \\
\text { databank set }\end{array}$ & $\begin{array}{l}\text { Foot and ankle } \\
\text { trauma } 2007 \text { to } \\
2009 \mathrm{~N}=75,664\end{array}$ & Mixed & $0.47 \%$ & Symptomatic & $0.28 \%$ & $0.21 \%$ & $0.49 \%$ \\
\hline $\begin{array}{l}\text { Griffiths } 2012 \\
\text { (Griffiths, } \\
\text { Matthews, } \\
\text { Pearce, \& } \\
\text { Calder, 2012) }\end{array}$ & $\begin{array}{l}\text { Retrospective } \\
\text { study }\end{array}$ & $\begin{array}{l}\text { Elective foot and } \\
\text { ankle surgery } \\
2003 \text { to } 2010 \\
(\mathrm{~N}=2,652) \text {. High } \\
\text { risk got LMWH } \\
\text { and excluded }\end{array}$ & $\begin{array}{l}\text { Unclear if all } \\
\text { immobilised }\end{array}$ & $\begin{array}{l}1,078 \text { got } 75 \mathrm{mg} \text { aspirin } \\
2003 \text { to } 2006 \text { and } 1,576 \\
\text { got nothing } 2007 \text { to } \\
2010\end{array}$ & Symptomatic & $0.27 \%$ & $0.15 \%$ & $0.42 \%$ \\
\hline $\begin{array}{l}\text { Adrichem } \\
2014 \text { (van } \\
\text { Adrichem et } \\
\text { al., 2014) }\end{array}$ & $\begin{array}{l}\text { Data from } \\
\text { large } \\
\text { population- } \\
\text { based case- } \\
\text { control study } \\
\text { (MEGA) }\end{array}$ & $\begin{array}{l}134 \text { VTE patients } \\
\text { and } 23 \text { controls }\end{array}$ & Cast & $\mathrm{N} / \mathrm{A}$ & $\begin{array}{l}\text { Entry to MEGA } \\
\text { case-control } \\
\text { study required } \\
\text { diagnosis of } \\
\text { symptomatic } \\
\text { event }\end{array}$ & & & $\begin{array}{l}\text { 8-fold } \\
\text { increased } \\
\text { risk of VTE } \\
\text { with LLI } \\
\text { 13-fold } \\
\text { increased } \\
\text { risk with } \\
\text { trauma }\end{array}$ \\
\hline $\begin{array}{l}\text { Vollans } 2015 \\
\text { (Vollans et al., } \\
2015 \text { ) }\end{array}$ & $\begin{array}{l}\text { Retrospective } \\
\text { review }\end{array}$ & $\begin{array}{l}\text { Tibial fractures } \\
(n=177)\end{array}$ & $\begin{array}{l}\text { Above knee cast } \\
\text { pre-operative, } \\
\text { then circular } \\
\text { frame fixation }\end{array}$ & $\begin{array}{l}\text { Chemoprophylaxis until } \\
\text { independent mobility } \\
\text { (could be almost } \\
\text { immediately post-op) }\end{array}$ & Symptomatic & $1.7 \%$ & $2.3 \%$ & $4.0 \%$ \\
\hline
\end{tabular}

VTE - Venous thromboembolism

ORIF - Open reduction and internal fixation

DVT - Deep vein thrombosis

PE - Pulmonary embolism

LMWH - Low molecular weight heparin

LLI - Lower limb immobilisation 
Table 3.2: Placebo arms of randomised controlled trials involving lower limb immobilisation

\begin{tabular}{|c|c|c|c|c|c|c|c|}
\hline Study author & $\begin{array}{l}\text { Type } \\
\text { of } \\
\text { study }\end{array}$ & Patient group & $\begin{array}{l}\text { Lower limb } \\
\text { immobilisation }\end{array}$ & Thromboprophylaxis? & $\begin{array}{l}\text { Symptomatic / } \\
\text { asymptomatic? }\end{array}$ & DVT rate & $\begin{array}{c}\text { Proximal / } \\
\text { distal }\end{array}$ \\
\hline $\begin{array}{l}\text { Kujath } 1993 \\
\text { (Kujath, } \\
\text { Spannagel, \& } \\
\text { Habscheid, 1993) }\end{array}$ & $\mathrm{RCT}$ & $\begin{array}{l}127 \text { patients with } \\
\text { lower limb injury } \\
\text { Placebo arm of } \\
\text { interventional study }\end{array}$ & Yes & None & $\begin{array}{l}\text { Screening by } \\
\text { compression US on } \\
\text { removal of cast }\end{array}$ & 16.5\% DVT & $\begin{array}{l}\text { There were } \\
27 \text { DVTs in } \\
\text { BOTH groups } \\
\text { of which } 24 \\
\text { where IDDVT }\end{array}$ \\
\hline $\begin{array}{l}\text { Spannagel } 1993 \\
\text { (Spannagel \& } \\
\text { Kujath, 1993) } \\
\end{array}$ & RCT & $\begin{array}{l}\text { Injury of lower limb. } \\
\mathrm{N}=127 \text { in control } \\
\text { arm }\end{array}$ & Plaster cast & None & $\begin{array}{l}\text { Compression US after } \\
\text { removal of cast, then by } \\
\text { phlebography }\end{array}$ & $16.5 \%$ DVT & Not stated \\
\hline $\begin{array}{l}\text { Kock } 1993(\mathrm{H} . \\
\text { Kock et al., 1993) }\end{array}$ & RCT & $\begin{array}{l}\text { Minor injuries } \\
\text { conservatively } \\
\text { treated with plaster } \\
\text { cast } \mathrm{LLI}, \mathrm{N}=126 \text { in } \\
\text { control group }\end{array}$ & Plaster cast & None & Not stated & $3 \% \mathrm{DVT}$ & Not stated \\
\hline $\begin{array}{l}\text { Reilmann } 1993 \\
\text { (Reilmann, } \\
\text { Weinberg, } \\
\text { Forster, \& } \\
\text { Happe, 1993) } \\
\end{array}$ & ? RCT & $\begin{array}{l}\text { Surgical outpatients } \\
N \text { unknown }\end{array}$ & Plaster cast & Unclear & Not known & $14 \%$ DVT & Not stated \\
\hline $\begin{array}{l}\text { Kock } 1995 \text { (H. J. } \\
\text { Kock, Schmit- } \\
\text { Neuerburg, } \\
\text { Hanke, Rudofsky, } \\
\text { \& Hirche, 1995) } \\
\end{array}$ & RCT & $\begin{array}{l}\text { Minor injuries } \\
\mathrm{N}=163 \text { in control } \\
\text { group }\end{array}$ & Plaster cast & None & $\begin{array}{l}\text { Venous occlusion } \\
\text { plethysmography, US } \\
\text { and Phlebography to } \\
\text { confirm suspected DVT }\end{array}$ & $4.3 \% \mathrm{DVT}$ & $\begin{array}{l}4 \text { IDDVT, } 3 \\
\text { proximal }\end{array}$ \\
\hline $\begin{array}{l}\text { Lassen } 2002 \text { (M. } \\
\text { R. Lassen, Borris, } \\
\text { \& Nakov, 2002) }\end{array}$ & RCT & $\begin{array}{l}\text { Leg fracture or TA } \\
\text { rupture } \\
N=188 \text { in controls }\end{array}$ & $\begin{array}{l}\text { Plaster cast or } \\
\text { brace }\end{array}$ & None & $\begin{array}{l}\text { Venography within one } \\
\text { week of cast removal }\end{array}$ & 19\%,(DVT) & $\begin{array}{l}25 \text { IDDVT } \\
10 \text { proximal }\end{array}$ \\
\hline
\end{tabular}




\begin{tabular}{|c|c|c|c|c|c|c|c|}
\hline $\begin{array}{l}\text { Jorgensen } 2002 \\
\text { (P. Jorgensen et } \\
\text { al., 2002) }\end{array}$ & RCT & $\begin{array}{l}\text { Outpatients } \\
\mathrm{N}=152\end{array}$ & Plaster cast & None & $\begin{array}{l}\text { Ascending unilateral } \\
\text { venography }\end{array}$ & $17 \%$ & $\begin{array}{l}17 \text { IDDVT } \\
1 \text { proximal }\end{array}$ \\
\hline $\begin{array}{l}\text { Lapidus } 2007 \text { (L. } \\
\text { J. Lapidus, } \\
\text { Rosfors, et al., } \\
2007 \text { ) }\end{array}$ & $\mathrm{RCT}$ & $\begin{array}{l}\text { Surgically treated } \\
\text { achilles tendon } \\
\text { rupture. } \\
\mathrm{N}=44 \text { in placebo } \\
\text { group }\end{array}$ & Equinus cast & None & $\begin{array}{l}\text { Duplex sonography at } 3 \\
\text { weeks and } 6 \text { weeks with } \\
\text { confirming } \\
\text { phlebography }\end{array}$ & $36 \%$ DVT & $\begin{array}{l}12 \text { distal } \\
3 \text { proximal }\end{array}$ \\
\hline $\begin{array}{l}\text { Lapidus } 2007 \text { (L. } \\
\text { J. Lapidus, } \\
\text { Ponzer, et al., } \\
2007 \text { ) }\end{array}$ & RCT & $\begin{array}{l}\text { Ankle injury with } \\
\text { operative } \\
\text { intervention ( } \mathrm{N}= \\
136 \text { in controls) }\end{array}$ & $\begin{array}{l}\text { Plaster cast or } \\
\text { othosis }\end{array}$ & $\begin{array}{l}\text { Control arm, but received } \\
\text { one week of dalteparin } \\
\text { prior to randomisation }\end{array}$ & $\begin{array}{l}\text { Unilateral phlebography } \\
\text { on cast removal }\end{array}$ & $\begin{array}{l}28 \% \\
\text { Proximal = } \\
3 \%\end{array}$ & $\begin{array}{l}13 \text { IDDVT } \\
4 \text { proximal }\end{array}$ \\
\hline $\begin{array}{l}\text { Goel } 2009 \text { (Goel } \\
\text { et al., 2009) }\end{array}$ & $\mathrm{RCT}$ & $\begin{array}{l}\text { Isolated fracture } \\
\text { below knee with } \\
\text { operative fixation, } \\
\mathrm{N}=111 \text { in placebo } \\
\text { group }\end{array}$ & Various & $\begin{array}{l}\text { None } \\
\text { STOPPED prior to meeting } \\
\text { necessary sample size }\end{array}$ & Bilateral venography & $12.6 \%$ DVT & $\begin{array}{l}\text { All }(\mathrm{N}=11) \\
\text { IDDVT }\end{array}$ \\
\hline $\begin{array}{l}\text { Selby } 2015 \\
\text { (Selby et al., } \\
2015 \text { ) }\end{array}$ & RCT & $\begin{array}{l}\text { Isolated leg } \\
\text { fractures requiring } \\
\text { surgery. } 131 \\
\text { placebo patients }\end{array}$ & $\begin{array}{l}129 \text { cast or } \\
\text { splint }\end{array}$ & None & $\begin{array}{l}\text { Bilateral US review of } \\
\text { proximal veins only at } \\
14 / 7 \text { and } 3 \text { months }\end{array}$ & $\begin{array}{l}2.3 \% \text { of } \\
\text { 'Clinically } \\
\text { Important } \\
\text { VTE' }\end{array}$ & All proximal \\
\hline
\end{tabular}

Shaded lines are included in the 2014 Cochrane review (Mark Testroote et al., 2014)

DVT - Deep vein thrombosis

RCT - Randomised controlled trial

IDDVT - Isolated distal deep vein thrombosis

US - Ultrasound

LLI - Lower limb immobilisation

TA - tendo-Achillis (Achilles tendon)

VTE - Venous thromboembolism 
Table 3.3: Prospective cohort studies involving lower limb immobilisation

\begin{tabular}{|c|c|c|c|c|c|c|c|}
\hline \multicolumn{8}{|c|}{ Symptomatic events } \\
\hline $\begin{array}{l}\text { Study } \\
\text { author }\end{array}$ & Patient group & $\begin{array}{l}\text { Lower limb } \\
\text { immobilisation }\end{array}$ & Thromboprophylaxis? & $\begin{array}{l}\text { Symptomatic / } \\
\text { asymptomatic? }\end{array}$ & DVT rate & $\begin{array}{l}\mathrm{PE} \\
\text { rate }\end{array}$ & $\begin{array}{l}\text { Overall } \\
\text { VTE rate }\end{array}$ \\
\hline $\begin{array}{l}\text { Mizel } \\
1998 \\
\text { (Mizel et } \\
\text { al., 1998) }\end{array}$ & $\begin{array}{l}\text { Patients undergoing surgery } \\
\text { of the foot and ankle } \\
N=2,733 \text {, }\end{array}$ & 1,444 immobilised & $\begin{array}{l}\text { Up to treating } \\
\text { physician, } 3 \% \text { aspirin } \\
\text { or warfarin }\end{array}$ & Symptomatic & $\begin{array}{l}0.22 \% \\
\text { Correlation with } \\
\text { LLI and NWB }\end{array}$ & $0.15 \%$ & $0.37 \%$ \\
\hline $\begin{array}{l}\text { Lapidus } \\
2013 \text { (L. } \\
\text { Lapidus et } \\
\text { al., 2013) }\end{array}$ & $\begin{array}{l}\text { Consecutive orthopaedic } \\
\text { procedures at one location, } \\
764 \text { Achilles tendon } \\
\text { ruptures }\end{array}$ & $\begin{array}{l}\text { Presumed equinus } \\
\text { cast }\end{array}$ & $\begin{array}{l}\text { None for Achilles } \\
\text { tendons }\end{array}$ & Symptomatic & $7.2 \%$ & & $7.2 \%$ \\
\hline $\begin{array}{l}\text { Selby } \\
2014 \\
\text { (Selby et } \\
\text { al., 2014) }\end{array}$ & $\begin{array}{l}\text { Patients with non-operative } \\
\text { tibia, fibular or ankle } \\
\text { fracture and patellar and } \\
\text { foot fractures (operative } \\
\text { and non-operative). } \\
N=1200,82 \%\end{array}$ & $\begin{array}{l}82 \% \text { cast or splint, } \\
\text { average } 6 \text { weeks }\end{array}$ & None & Symptomatic & $\begin{array}{l}0.4 \% \text { (3 IDDVT, } 3 \\
\text { proximal) }\end{array}$ & $0.2 \%$ & $0.6 \%$ \\
\hline $\begin{array}{l}\text { Study } \\
\text { author }\end{array}$ & Patient group & LLI? & Thromboprophylaxis? & $\begin{array}{l}\text { Symptomatic / } \\
\text { asymptomatic? }\end{array}$ & DVT rate & $\begin{array}{l}\mathrm{PE} \\
\text { rate }\end{array}$ & $\begin{array}{l}\text { Overall } \\
\text { VTE rate }\end{array}$ \\
\hline $\begin{array}{l}\text { Solis } \\
\text { 2002(Solis } \\
\text { \& Saxby, } \\
2002 \text { ) }\end{array}$ & $\begin{array}{l}\text { Consecutive patients } \\
\text { undergoing foot and ankle } \\
\text { surgery ( } \mathrm{N}=201)\end{array}$ & Not mentioned & Not mentioned & $\begin{array}{l}\text { Screening by US at } \\
\text { first post-operative } \\
\text { visit }\end{array}$ & $3.5 \%$ & & $3.5 \%$ \\
\hline
\end{tabular}




\begin{tabular}{|c|c|c|c|c|c|c|c|}
\hline $\begin{array}{l}\text { Riou } 2007 \\
\text { (Riou et } \\
\text { al., 2007) }\end{array}$ & $\begin{array}{l}\text { Non-surgical lower limb } \\
\text { injury below the knee. } \\
N=2761\end{array}$ & $\begin{array}{l}7 \text { to } 90 \text { days, } \\
\text { variable types }\end{array}$ & Up to physicians & $\begin{array}{l}\text { Asymptomatic US } \\
\text { screening after } \\
\text { immobilisation } \\
\text { removal }\end{array}$ & $6.1 \%$ & $0.3 \%$ & $6.4 \%$ \\
\hline $\begin{array}{l}\text { Patil } 2007 \\
\text { (Patil, } \\
\text { Gandhi, } \\
\text { Curzon, \& } \\
\text { Hui, } \\
\text { 2007) }\end{array}$ & $\begin{array}{l}100 \text { non-operative ankle } \\
\text { fracture patients without } \\
\text { prophylaxis }\end{array}$ & Plaster cast & None & $\begin{array}{l}\text { Screening by US on } \\
\text { removal of cast }\end{array}$ & $\begin{array}{l}5 \% \text { DVT, all } \\
\text { asymptomatic, two } \\
\text { proximal }\end{array}$ & & $5.0 \%$ \\
\hline
\end{tabular}

Records review

\begin{tabular}{|c|c|c|c|c|c|c|c|}
\hline $\begin{array}{l}\text { Study } \\
\text { author }\end{array}$ & Patient group & $\begin{array}{l}\text { Lower Limb } \\
\text { Immobilisation }\end{array}$ & Thromboprophylaxis? & $\begin{array}{l}\text { Symptomatic / } \\
\text { asymptomatic? }\end{array}$ & DVT rate & $\begin{array}{l}\mathrm{PE} \\
\text { rate }\end{array}$ & $\begin{array}{l}\text { Overall } \\
\text { VTE rate }\end{array}$ \\
\hline $\begin{array}{l}\text { Haque } \\
2015\end{array}$ & $\begin{array}{l}\text { Ankle fracture, non- } \\
\text { operative management, }\end{array}$ & Plaster cast & $\begin{array}{l}148 \text { low risk, no } \\
\text { intervention, } 52 \text { high }\end{array}$ & $\begin{array}{l}\text { Review of records for } \\
\text { confirmed events }\end{array}$ & $\begin{array}{l}0.5 \%, \text { one low risk } \\
\text { patient IDDVT }\end{array}$ & & $0.5 \%$ \\
\hline
\end{tabular}

(Haque \& N=200

risk got rivaroxaban

Davies,

2015)

VTE - Venous thromboembolism

LLI - Lower limb immobilisation

NWM - non weight-bearing

IDDVT - isolated distal deep vein thrombosis

US - ultrasound

DVT - Deep vein thrombosis 
From the available literature, one might infer that at least in Achilles tendon injuries with plaster cast immobilisation the rates of VTE are consistently higher compared to many other patients groups in this clinical setting. Achilles tendon injuries have reported symptomatic VTE rates of $6.3 \%$ in a retrospective review of data (B. Healy, Beasley, et al., 2010) and 7.2\% in a prospective cohort study (L. Lapidus et al., 2013). In the RCT undertaken by Lapidus and colleagues, $36 \%$ of participants in the placebo arm of the trial had DVT detected through screening (L. J. Lapidus, Rosfors, et al., 2007). These comparatively high numbers are offset only by a large database review of Achilles tendon ruptures that reported confirmed VTE events of only $0.8 \%$ (A Patel et al., 2012), which appears more consistent with those rates reported after foot and ankle surgery. Whether there is a systemic difference between the data of the patients in the Patel study, or the management of Achilles tendon patients between the studies is unclear. The Patel data also included partial tendon ruptures which may have been managed with heel-wedges within weight-bearing moonboots, as is sometimes the case at Wellington Hospital Fracture Clinic, but the proportion of patients that may have been managed in this way is uncertain.

In contrast to lower limb immobilisation, the high risk of VTE with major orthopaedic surgery has been well documented, with radiological screening VTE rates of 44 to $90 \%$ reported following hip and knee joint replacement surgery without prophylactic measures (Falck-Ytter et al., 2012; L. N. Jorgensen, Wille-Jorgensen, \& Hauch, 1993; Salazar, Malaga, \& Malasquez, 2010; Turpie et al., 1986). Over the last 40 years clinical trials demonstrating the efficacy and safety of prophylactic anticoagulant regimens in major orthopaedic surgery have been undertaken, resulting in a reduction of VTE identified with radiological screening to between $6 \%$ and $7 \%$. This has led to the development of robust guidelines for VTE prophylaxis in the setting of elective orthopaedic surgery (Falck-Ytter et al., 2012). The role of prophylactic anticoagulation in the more ambulatory setting of lower limb immobilisation, despite the proven efficacy of LMWH in reducing the risk of VTE in this clinical setting is less clear.

To place the VTE risk associated with lower limb immobilisation in a current clinical perspective, I considered it informative to determine the relative risk of VTE associated with 
lower limb immobilisation in the Wellington region, and to compare the proportion of all regional VTE cases associated with lower limb immobilisation with that due to other potentially preventable risk factors.

The Healy study of 2010 (B. Healy, Beasley, et al., 2010) is a review of patients treated for Achilles tendon injury at Wellington Hospital Outpatients fracture clinic, and so is an assessment of the incidence of symptomatic VTE events within this patient group from my own geographical area of clinical practice. While the rates of symptomatic presentation of VTE events was low (6.3\%) from an orthopaedic perspective, each of the affected patients underwent diagnostic procedures, variable periods of treatment on anticoagulation therapy and were at risk of developing the sequalae of VTE due to a predictable, and possibly preventable high risk VTE event.

The combined case-control analysis undertaken in Chapter Two provided data that enabled me to consider the burden of VTE events in lower limb immobilisation as a proportion of all VTE events presenting to the Wellington Hospital VTE outpatient clinics. Considering presentations of VTEs in this context allows categorisation of all VTE patients into those with modifiable and non-modifiable risk factors and prioritisation of patient groups that might benefit from interventions such as education, early mobilisation and prophylaxis. The following study outlines these findings. 


\section{STUDY THREE: Lower limb immobilisation and venous thromboembolism risk: Combined Case-control studies}

\section{Hypothesis}

The hypothesis of this study was that lower limb immobilisation is associated with increased risk of venous thromboembolism (VTE).

\section{Primary outcome variable}

The primary outcome variable was the odds ratio for association between lower limb immobilisation and VTE.

\section{Secondary outcome variable}

The secondary outcome variable was the number of VTE patients that had undergone lower limb immobilisation in the four weeks prior to their VTE event as a proportion of all VTE patients included in the Healy and my own case-control studies (I Braithwaite, Maher, et al., 2016; B. Healy, Levin, et al., 2010).

\section{Methods}

This was an analysis of the combined data from Study One already reported (I Braithwaite et al. 2016), and the Healy case-control study published in 2010 (B. Healy, Levin, et al., 2010). Cases comprised 396 patients with radiologically confirmed deep vein thrombosis (DVT) or pulmonary embolism (PE) attending outpatient VTE clinics. Controls comprised 197 inpatients in the coronary care unit and 200 outpatients treated for upper limb injuries in the fracture clinic. Statistical analysis was by logistic regression analysis of combined data from two case-control studies which utilised similar methodology, to investigate the association of risk factors with VTE in adults aged 18 to 65 years, with a specific focus on lower limb immobilisation. The proportion of VTE patients who had undergone lower limb immobilisation was also calculated. 


\section{Patients and Methods}

Data from two case-control studies utilising similar methodology and questionnaires (Appendix II, Appendix III) to investigate the VTE risk associated with occupational seated immobility and other factors were combined (I Braithwaite, Maher, et al., 2016; B. Healy, Levin, et al., 2010). In an initial study Healy and colleagues obtained data from 196 consecutive cases attending the CCDHB VTE clinics and 197 controls admitted to the CCDHB CCU between February 2007 and February 2009 (B. Healy, Levin, et al., 2010). In my casecontrol study I obtained data from 200 cases attending the CCDHB VTE clinics and 200 controls attending the CCDHB fracture clinic with upper limb injuries between October 2011 and January 2013 (I Braithwaite, Maher, et al., 2016).

In both case-control studies, for inclusion as a case the patients needed to be aged between 18 and 65 years and have a confirmed diagnosis of VTE within the last six months. A diagnosis of VTE required confirmation by compression Doppler US, lung ventilation / perfusion scan, or helical CTPA. Patients who presented with an arterial thromboembolic event or who had superficial thrombophlebitis without extension into the deep venous system were excluded. The CCU controls were any inpatient aged between 18 and 65, admitted for any reason other than VTE, and had no VTE or clinical suspicion of VTE in the previous six months. The upper limb injury controls were eligible if they were aged between 18 and 65, had suffered a traumatic upper limb injury within the previous six months and had no VTE, or clinical suspicion of VTE, within the same time frame.

Common data collected included age, sex, information about the index event and risk factors for VTE, utilising similar questionnaires administered by the study investigators. For lower limb immobilisation, the date of initial immobilisation, the duration of immobilisation (where documented), and reason for immobilisation (fracture, fracture followed by surgery, elective surgery alone, Achilles tendon rupture or other soft tissue injury) was documented. Thrombophilia screens were only inconsistently available in some cases, and were not available for the controls, and therefore were not included as a risk factor in the present study. 
Both case-control studies received local health and research ethics committee approval and informed consent was obtained from all study participants, prior to any study procedures being undertaken. 


\section{Statistical Methods}

Univariate logistic regression was used to measure the strength of the association between case - control status and other explanatory variables. I originally intended to undertake a multivariate logistic regression adjusted for pre-specified confounding variables: age, body mass index $(\mathrm{BMI})>30 \mathrm{~kg} / \mathrm{m}^{2}$, sex, hormonal contraceptive use, a personal or family history of VTE, and any of the following within the 28 days prior to the development of symptoms of the index event; orthopaedic surgery, non-orthopaedic surgery and long distance travel. However, due to the sparse data for the surgical variables, the confidence intervals for the associations in univariate analysis were very wide and multivariate analysis was not attempted.

The percentage of cases that had undergone lower limb immobilisation in the four weeks prior to their index VTE event was calculated. The difference between the percentages of cases and controls with a potentially preventable risk factor was also calculated.

\section{Power Calculation}

This study is a retrospective exploration of pre-existing data and there were no predetermined power calculations. The original case-control studies were designed to assess the risk of association for VTE with prolonged work- and computer-related immobility. In the study by Healy and colleagues trauma or cast immobility had an odds ratio (OR) of association with VTE of 10.3 (95\% Cl 4.4 to $24.0, \mathrm{P}<0.001$ ) (B. Healy, Levin, et al., 2010), and in the Braithwaite and colleagues study, trauma or cast immobility had an odds ratio of association with VTE of 15.9 ( $95 \% \mathrm{Cl} 6.2$ to $40.7, \mathrm{P}<0.001$ ) (I Braithwaite, Maher, et al., 2016). 


\section{Results}

396 cases and 397 controls were analysed. Characteristics of all participants are shown in Table 3.4.

Table 3.4: Characteristics of study participants.

Mean (SD)

\begin{tabular}{|c|c|c|}
\hline & $\begin{array}{l}45.9(12.4) \\
N=396\end{array}$ & $\begin{array}{l}47.1(11.8) \\
\mathrm{N}=395\end{array}$ \\
\hline Age (years)* & Case N/N (\%) & Control N/N (\%) \\
\hline $\mathrm{BMI}>30 \mathrm{~kg} / \mathrm{m}^{2 *}$ & $151 / 390(38.7)$ & $137 / 395(34.7)$ \\
\hline Male sex* & $207 / 396(52.3)$ & $252 / 397(63.5)$ \\
\hline Hormonal contraceptive use* & $51 / 396(12.9)$ & $10 / 397(2.5)$ \\
\hline Personal history VTE* & $69 / 396(17.4)$ & $7 / 397(1.8)$ \\
\hline Family history VTE* & $82 / 396(20.7)$ & 41/397 (10.3) \\
\hline Long distance travel* & $57 / 396(14.4)$ & 20/397 (5.0) \\
\hline $\begin{array}{l}\text { Prolonged work- and computer-related seated } \\
\text { immobility }\end{array}$ & 63/396 (15.9) & $48 / 397(12.1)$ \\
\hline Cancer & $4 / 396(1.0)$ & $5 / 397(1.3 \%)$ \\
\hline Non-orthopaedic surgery* & $30 / 396(7.6)$ & $5 / 397(1.3)$ \\
\hline Orthopaedic surgery* & $38 / 396(9.6)$ & $1 / 397(0.3)$ \\
\hline $\begin{array}{l}\text { - Orthopaedic surgery with consequent lower } \\
\text { limb immobilisation }\end{array}$ & $15 / 396(3.8)$ & 0/397 (0) \\
\hline $\begin{array}{l}\text { - Orthopaedic surgery without consequent lower } \\
\text { limb immobilisation* }\end{array}$ & $23 / 396(5.8)$ & $1 / 397(0.3)$ \\
\hline Lower limb immobilisation* & $62 / 396(15.7)$ & $1 / 397(0.3)$ \\
\hline - Lower limb immobilisation -without surgery* & 47/396 (11.9) & $1 / 397(0.3)$ \\
\hline Second case-control study* & $200 / 396(50.5)$ & 200/397 (50.4) \\
\hline
\end{tabular}

SD - Standard Deviation

$\mathrm{BMI}$ - Body Mass Index

VTE - Venous thromboembolism

Prolonged work-and computer-related seated immobility - In the 28 days before the index event, had at least one episode of sitting for work and / or recreational computer use for a minimum of 10 hours in a day, with a period of at least 2 hours seated without getting up.

*Included in the univariate regression analyses 
Of the $38 / 396$ (9.6\%) cases that had undergone orthopaedic surgery, 15 were then immobilised in a lower limb cast. The 23 cases of orthopaedic surgery without subsequent cast immobilisation included 6 total hip joint replacement surgeries, 5 total knee joint replacement surgeries and 4 other knee interventions ( 2 washouts, one arthroscopy, and one ligament repair). The remaining operations were spinal or involving the upper limbs. There was one control that underwent orthopaedic surgery without consequent lower limb immobilisation.

There were 62/396 (15.7\%) cases reporting lower limb immobilisation in the four weeks prior to their index event. There was one control reporting lower limb immobilisation due to a fractured ankle, which required no surgical intervention. The cause of lower limb immobilisation for cases and controls is outlined in Table 3.5.

Table 3.5: Clinical situation for lower limb immobilisation in cases and controls

\begin{tabular}{lll}
\hline & Cases & Controls \\
& $\mathbf{N}$ (surgical intervention) & $\mathbf{N}$ (surgical intervention) \\
\hline Fractures & $38(7)$ & $1(0)$ \\
Achilles tendon injuries & $16(2)$ & $0(0)$ \\
Elective operation & $5(5)$ & $0(0)$ \\
Soft tissue injury & $3(1)$ & $0(0)$ \\
\hline Total reporting lower limb immobilisation & $\mathbf{6 2 ( 1 5 )}$ & $\mathbf{1 ( 0 )}$ \\
\hline
\end{tabular}




\section{Lower limb immobilisation and venous thromboembolism}

The univariate associations between VTE and the potential risk factors are shown in Table 3.6. There was a significant association between lower limb immobilisation and VTE with an OR for association of $73.1(95 \% \mathrm{Cl} 10.1$ to $530, \mathrm{P}<0.001)$. The wide confidence interval is reflective of the single control that underwent lower limb immobilisation in the 28 days prior to their index event. Positive associations were shown for lower limb immobilisation without surgery, and for orthopaedic surgery with and without subsequent lower limb immobilisation, with wide confidence intervals again reflective of the infrequent traits in the control group.

Table 3.6: Univariate Odds Ratios $(95 \% \mathrm{Cl})$ for association of risk factors and VTE.

\begin{tabular}{|c|c|}
\hline & Odds ratio ( $95 \% \mathrm{Cl}$; $\mathrm{P}$ value) \\
\hline Age per decade older & $0.92(0.82$ to $1.04 ; 0.18)$ \\
\hline BMI $\left(\mathrm{Kg} / \mathrm{m}^{2}\right)>30$ & 1.19 (0.89 to $1.59 ; 0.24)$ \\
\hline Male compared to female & $0.64(0.48$ to $0.85 ; 0.002)$ \\
\hline Oral contraceptive use & $5.66(2.83$ to $11.3 ;<0.001)$ \\
\hline Personal history VTE & 11.7 (5.3 to $25.8 ;<0.001)$ \\
\hline Family history VTE & $2.30(1.53$ to $3.45 ;<0.001)$ \\
\hline Long distance travel* & $3.14(1.85$ to $5.35 ;<0.001)$ \\
\hline Non-orthopaedic surgery & $6.50(2.49$ to $16.9 ;<0.001)$ \\
\hline Orthopaedic surgery & $42.5(5.81$ to $311 ;<0.001)$ \\
\hline $\begin{array}{l}\text { - Orthopaedic surgery with consequent lower limb } \\
\text { immobilisation }\end{array}$ & N/A \\
\hline $\begin{array}{l}\text { - Orthopaedic surgery without consequent lower } \\
\text { limb immobilisation }\end{array}$ & $24.7(3.32$ to $184 ; 0.002)$ \\
\hline Lower limb immobilisation & $73.1(10.1$ to $530 ;<0.001)$ \\
\hline - $\quad$ Lower limb immobilisation -without surgery & $52.6(7.23$ to $383 ;<0.001)$ \\
\hline Second case-control study versus first case-control study & $1.03(0.78$ to $1.36 ; 0.86)$ \\
\hline $\begin{array}{l}\mathrm{Cl} \text { - confidence interval } \\
\mathrm{BMI} \text { - body mass index } \\
\mathrm{VTE} \text { - venous thromboembolism }\end{array}$ & \\
\hline
\end{tabular}




\section{Lower limb immobilisation and other risk factors for venous thromboembolism}

Of the 62 cases with lower limb immobilisation, 19 (31\%) had no other established risk factors, 24 (39\%) had one other risk factor, 18 (29\%) had two other risk factors and one (2\%) had four other risk factors (Figure 3.1). The most common additional risk factor in those with lower limb immobilisation was obesity, which was reported in 27/62 (43.5\%), compared with 124/328 (38\%) of the other cases and 137/395 (34.7\%) controls. Excluding obesity, most cases with lower limb immobility had no identifiable risk factor.

Figure 3.1: The proportion of cases with cast immobilisation in the four weeks prior to their index VTE event $(n=62)$ with other risk factors for VTE. Cases may have had more than one other risk factor.

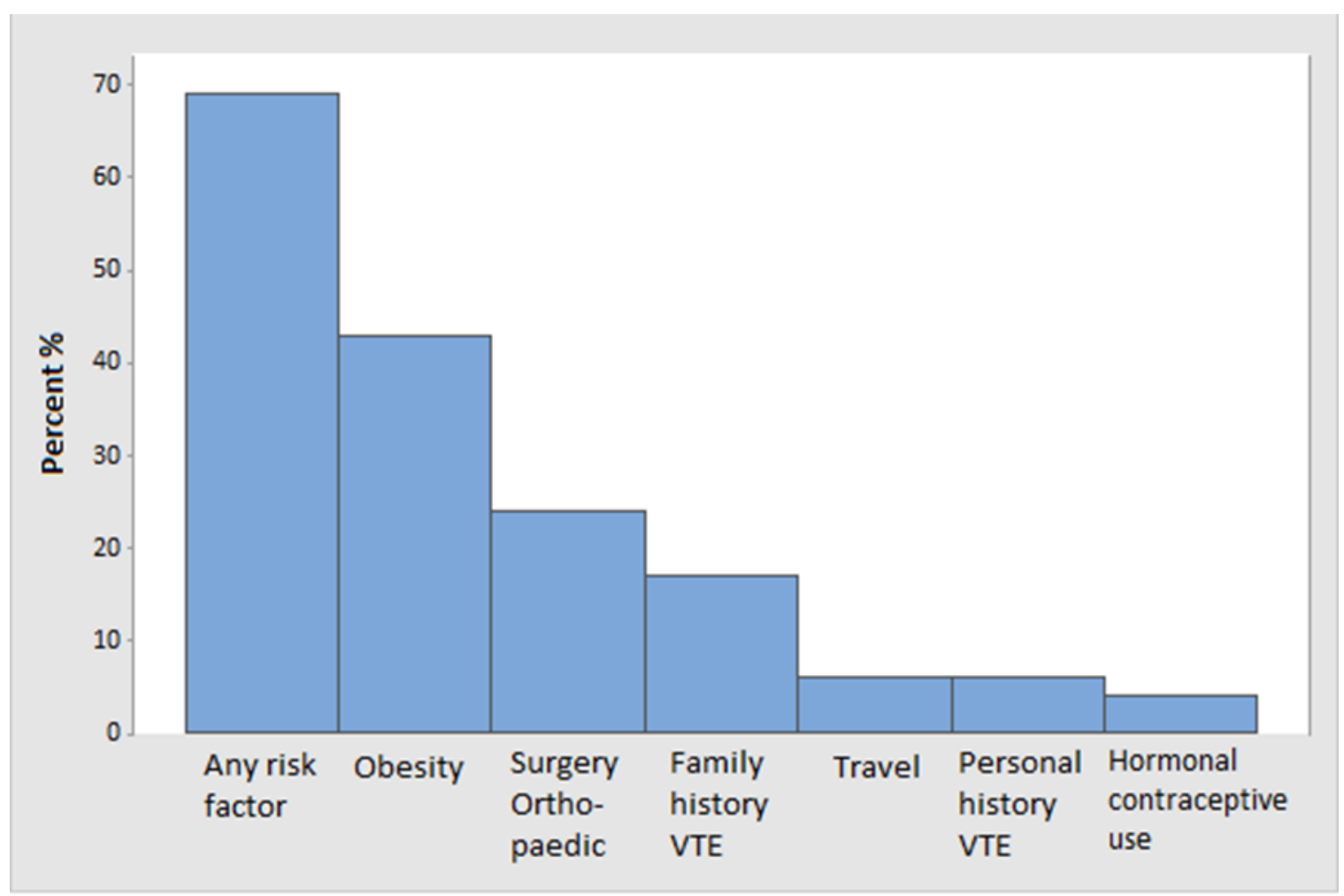




\section{Proportion of cases and controls with a potentially preventable risk factor for venous}

\section{thromboembolism}

The difference between the percentages of cases and controls with a potentially preventable risk factor is shown in Table 3.7. Lower limb immobility was the most common potentially preventable risk factor; present in $15.4 \%$ more cases than controls. The subgroup of lower limb immobilisation without surgery was the second most common potentially preventable risk factor, present in $11.6 \%$ more cases than controls. By comparison, orthopaedic surgery without lower limb immobilisation, (predominantly knee and hip joint replacement surgery), and non-orthopaedic surgery were present in $5.5 \%$ and $6.3 \%$ more cases than controls respectively.

Table 3.7: The difference between the percentage of cases and controls with a potentially preventable risk factor for VTE.

\begin{tabular}{|c|c|c|c|c|}
\hline & $\begin{array}{l}\text { Cases } \\
(\%)\end{array}$ & $\begin{array}{l}\text { Controls } \\
(\%)\end{array}$ & $\begin{array}{l}\text { Cases minus } \\
\text { controls (\%) }\end{array}$ & $\begin{array}{l}95 \% \mathrm{Cl} \text { for difference in } \\
\text { percentages } \\
\text { ( } \mathrm{P} \text { value) }\end{array}$ \\
\hline $\mathrm{BMI}\left(\mathrm{Kg} / \mathrm{m}^{2}\right)>30$ & 38.7 & 34.7 & 4.0 & -2.7 to $10.7(0.245)$ \\
\hline Oral Contraceptive use & 12.9 & 2.5 & 10.4 & 6.7 to $14.1(<0.0001)$ \\
\hline Long distance travel* & 14.4 & 5.0 & 9.4 & 5.2 to $13.5(<0.0001)$ \\
\hline $\begin{array}{l}\text { Prolonged work- and computer- } \\
\text { related seated immobility }\end{array}$ & 15.9 & 12.1 & 3.8 & -1.0 to $8.6(0.1231)$ \\
\hline Non-orthopaedic surgery & 7.6 & 1.3 & 6.3 & 3.4 to $9.2(<0.0001)$ \\
\hline Orthopaedic surgery & 9.6 & 0.3 & 9.3 & 6.3 to $12.3(<0.0001)$ \\
\hline - with lower limb immobilisation & 3.8 & 0 & 3.8 & 1.9 to $5.7(<0.0001)$ \\
\hline $\begin{array}{l}\text { - without lower limb } \\
\text { immobilisation }\end{array}$ & 5.8 & 0.3 & 5.5 & 3.1 to $7.9(<0.0001)$ \\
\hline Lower limb immobilisation & 15.7 & 0.3 & 15.4 & 11.6 to $19.2(<0.0001)$ \\
\hline - without surgery & 11.9 & 0.3 & 11.6 & 8.3 to $14.9(<0.0001)$ \\
\hline
\end{tabular}




\section{Discussion}

This analysis has shown that lower limb immobilisation is associated with a markedly increased risk of VTE, and is the most common preventable risk factor for VTE in this sample. One in seven cases of VTE underwent lower limb immobilisation in the four weeks prior to the index VTE event, a proportion which was greater than orthopaedic surgery without subsequent lower limb immobilisation, and non-orthopaedic surgery combined. These findings suggest that the greatest opportunity to reduce the burden of VTE in this population is to reduce the VTE risk in the clinical setting of lower limb immobilisation. Based on current evidence, this would require the prescription of pharmacological preventive measures, such as LMWH which has been proven to substantially reduce VTE the risk in lower limb immobilisation without increasing the risk of bleeding (Ettema, Kollen, Verheyen, \& Büller, 2008; Mark Testroote et al., 2014).

The finding of greatly increased risk of VTE with lower limb immobilisation is broadly in line with the few studies that have attempted an assessment of this same risk factor. Two casecontrol studies assess lower limb immobilisation as a specific risk factor for VTE. Using data from the MEGA population based case-control study, van Adrichem and colleagues have found that lower limb immobilisation conferred an odds ratio for association with VTE of 56 within three months of cast application in patients aged 18 to 70 years (van Adrichem et al., 2014). They also found an increased risk of VTE in conservatively treated patients compared to surgically treated patients, and in trauma patients compared to non-trauma patients. The Age and Thrombosis - Acquired and Genetic risk factors in the Elderly (AT-AGE) study, found an odds ratio for association of plaster casts with VTE of 6.2 in patients more than 70 years of age. An age in excess of 65 years was an exclusion criteria for the case-control studies analysed in our own study, so a direct comparison of our results cannot be made with the AT-AGE study.

Huerta reports an odds ratio for association with VTE of 33.2 with lower extremity fracture in a prospective cohort study with a nested case-control analysis of a primary healthcare population in the United Kingdom (Huerta et al., 2007). It is unclear however, whether all cases in the Huerta study underwent lower limb immobilisation so a direct comparison with 
our findings cannot be made. K Sonnevi has found that in a subset of women aged 18 to 64 years included in the Swedish Thrombo Embolism Hormone Study (TEHS) who agreed to further follow up (Hypo-TEHS), exposure to surgery and / or a lower limb cast had an OR for association with VTE of 16 and 66 in women with no family history of VTE and a positive family history of VTE respectively (Sonnevi, 2013). The lack of separation between exposure to a surgical procedure and lower limb casting makes comparison with our results infeasible. There are a number of large case-control studies that investigate risk factors for VTE, but many do not reported data specific to lower limb immobilisation (J. Heit et al., 2002; van Stralen, Rosendaal, \& Doggen, 2008).

The lack of studies assessing lower limb immobilisation as an independent variable for VTE risk may reflect a lack of awareness of its importance despite observational studies which have reported rates of VTE as high as $35 \%$ in which radiological screening has been undertaken (L. J. Lapidus, Rosfors, et al., 2007), or it may have been overlooked due to the potentially large proportion of patients who are treated in the outpatient setting and are thus not considered to have the risks for VTE that are expected in the context of large surgical procedures.

That the risk of VTE in association with lower limb immobilisation was similar regardless of whether there was prior surgery, suggests that prolonged cast immobilisation is the major independent risk factor for VTE. This is consistent with the reports that the risk of VTE following Achilles tendon rupture is similar regardless of surgical interventions (B. Healy, Beasley, et al., 2010; Nilsson-Helander, Thurin, Karlsson, \& Eriksson, 2009; A Patel et al., 2012). For example, in a study using radiological screening, the incidence of DVT after Achilles tendon rupture was $39 \%$ in those receiving non-surgical treatment and $29 \%$ in those receiving surgical treatment (Nilsson-Helander et al., 2009).

Importantly, this study identified that in our population of adults aged 18 to 65 years, lower limb immobilisation was the most common potentially preventable risk factor for VTE, being present in one in seven cases treated in our outpatient clinic. Of all the risk factors analysed, lower limb immobilisation had the greatest proportional difference between cases and controls (15.4\%). This difference was greater than the proportional difference seen in 
orthopaedic surgery without subsequent lower limb immobilisation such as hip and knee arthroplasty (5.5\%) and non-orthopaedic surgery (6.3\%) combined. Lower limb immobilisation was also more commonly identified than hormonal contraceptive use or long distance travel, which were present in $10.4 \%$ and $9.4 \%$ more cases than controls respectively.

This observation that the greatest potentially preventable burden from VTE is due to lower limb immobilisation raises the issue of pharmacological preventive measures. The efficacy of aspirin in reducing DVT was proven in the Pulmonary Embolism Prevention trial (Pulmonary Embolism Prevention (PEP) Trial Collaborative Group, 2000), however, there have been no placebo-controlled studies of the efficacy of aspirin in the prevention of VTE in the setting of lower limb immobilisation. There has been one study in which the efficacy of aspirin 500mg twice daily was compared to a LMWH in lower limb immobilisation (Gehling et al., 1998). In this study 9/143 (6.3\%) of patients on LMWH were screened radiologically and diagnosed with DVT, and 7/144 (4.8\%) of patients randomised to aspirin were diagnosed with DVT. In this study lower limb immobilisation included bandages as well as casts and weight-bearing capacity of participants was not described.

Two systematic reviews and meta-analyses have reported that pharmacological prophylaxis with LMWH reduces the VTE risk in lower limb immobilisation by $49 \%$ to $58 \%$, without increasing the risk of bleeding (Ettema et al., 2008; Mark Testroote et al., 2014). These findings are robust, with the efficacy confirmed in methodologically superior trials encompassing tendon ruptures and fractures. In this light, it is important that randomised controlled trials comparing LMWH either with aspirin or with the new factor Xa inhibitors are now undertaken. Factor Xa inhibitors may be preferred to aspirin in fact, as they have a better efficacy and similar safety profile compared with LMWH in the prevention of VTE following hip and knee replacement surgery (B. Eriksson et al., 2008; M. Lassen et al., 2008).

A related consideration is whether it might be possible to target prophylaxis to patients undergoing lower limb immobilisation who are identified of being at high risk of VTE following a screening assessment for recognised risk factors. However, this study shows that apart from obesity, measured by a BMI of $>30 \mathrm{~kg} / \mathrm{m}^{2}$, which was present in just over 
$40 \%$ of the cases with lower limb immobilisation, and $35 \%$ of controls, most cases with lower limb immobilisation did not have a risk factor that would be identified by clinical history such as personal or family history of VTE, medical conditions including cancer, long distance travel or hormonal contraceptive use. As a result, universal application of pharmacological preventive measures might be preferable to limiting prophylaxis to those with recognised risk factors.

There are some methodological issues to take into account with this study. Both the casecontrol studies that contributed to this analysis were designed and powered to detect an association between prolonged work- and computer-related immobility and VTE. As a result the data may not be adequately powered to detect the true odds for association between lower limb immobilisation and VTE. The paucity of controls that underwent lower limb immobilisation gave rise to large confidence intervals that made it injudicious to undertake a multivariate analysis. While both studies used the same criteria for case selection, the controls came from two different sources; the first from admissions to a coronary care unit and the second from outpatients managed for an upper limb injury in a fracture clinic. In the context of lower limb immobilisation and VTE it is unlikely that two sources for the control group would bias the findings, particularly as both groups had very low rates of lower limb immobilisation with or without surgery.

It would have been informative to have recorded use of pharmacological preventive measures for all cases, however without data on their use in the whole exposed population, any results would have been difficult to interpret. As the focus of the case-control studies was on work- and computer-related immobility, the upper age limit was 65 years, and as a result, the odds ratio and proportion calculated for risk factors may not reflect the rate observed in the elderly population.

\section{Conclusions}

Lower limb immobilisation was associated with a 70-fold increased risk of VTE and was a risk factor in $17 \%$ of those treated for VTE. Consideration should be given to prescribing prophylaxis for VTE in the setting of lower limb immobilisation, as this represents a common, possibly preventable risk factor for VTE. 


\section{Chapter Four: Interventions for the prevention of venous thromboembolism in sedentary workers}

Having established that there is an increasing body of evidence for the risk of VTE in the setting of the modifiable behaviour of prolonged work- and computer-related seated immobility, it is important to assess possible interventions to reduce or modify this risk in some way.

When considering possible interventions, the clinical setting needs to be taken into account. For example, while it may be possible to change some aspects of a predominantly sedentary workplace to modify the behaviour of prolonged sitting, it is important to consider the impact of changes on that environment and possible effects on workplace productivity.

\section{Interventions to reduce everyday sedentary behaviours}

There have been a number of interventions designed to reduce sedentary behaviours documented in the medical literature, although most have been focused on lifestyle interventions in at risk groups. For example the benefits of brief lifestyle intervention in reducing self-reported sedentary time in 3,232 Diabetes Prevention Programme participants have been reported (Rockette-Wagner et al., 2015). After brief discussion about reducing sedentary activities such as television viewing time and advocating self-monitoring of sedentary time, participants reduced television viewing time by 22 minutes per day in the first year compared to seven minutes and three minutes in the placebo and metformin groups respectively.

However, it has also been found that adults aged 18 to 40 years who were overweight or obese did not reduce their sedentary time as measured by accelerometer after a three hour group-based education intervention, use of a self-monitoring tool and follow-up motivational phone calls (Biddle et al., 2015). These two studies highlight the variability of results that can be found in such interventions. 


\section{Interventions to reduce sedentary behaviour at work}

More recently studies evaluating the effectiveness and feasibility of interventions designed to reduce sedentariness in the workplace have been published. Thompson and colleagues assessed 'walking workstations' in a sample of 25 employee volunteers in the Executive Health Programme at the Mayo Clinic comprising nurses, clinical assistants and secretaries. In this small trial daily steps per participant were increased during the working day.

Participant feedback was that the workstations were feasible in their environment, although they felt there was an initial drop in productivity as people got used to the workstations (Thompson, Foster, Eide, \& Levine, 2008).

Straker and colleagues have reported a feasibility study of 30 office workers (including 15 touch typists) using walking treadmills and cycling workstations. They found that computer task performance was lower when walking and cycling compared with sitting, with mouse performance being the most affected and concluded that active workstations may not be suitable for mouse intensive work (Straker, Levine, \& Campbell, 2009).

John and colleagues reached similar conclusions, finding that compared with seated computer work, treadmill desks caused a reduction in fine motor skills and math problem solving. In all, it has generally been concluded that the most feasible change in workstations that might improve activity levels would be implementation of 'sitting to standing' workstations, which might result in a reduction of sedentary time however, the efficacy of this potentially costly option has not been yet been assessed in a large workplace environment, and the possible effect or otherwise on VTE risk or the venous haemodynamics of the lower limb is not known.

A meta-analysis of workplace interventions to improve employee activity found that generally, most interventions tended to have transient effects and were quite resource intensive (Dugdill, Brettle, Hulme, McCluskey, \& Long, 2008), raising the issue of the costeffectiveness of possible interventions such as walking workstations and sitting to standing desks. 


\section{Interventions for repetitive strain injury}

Studies addressing specific workplace related health problems are fewer and have been mostly focused on the prevention of neck-shoulder and hand-arm disorders; now known as repetitive strain injury (RSI) associated with protracted computer use. RSI-related injuries in the workplace is now a well-recognised adverse effect of computer-related work, and it is increasingly common practice to fit out offices with ergonomic chairs, computers and workstations, and in larger work-centres, to implement reminders on computer systems for staff to undertake regular exercises for the alleviation of muscle and ligament strain associated with computer use (New Zealand Accident Compensation Corporation (ACC), 2013).

\section{Interventions to improve lower limb haemodynamics while sitting}

There is only one study of interventions to improve lower limb haemodynamics in seated participants, and this is in the context of prolonged air travel rather than the working environment (Hitos et al., 2007). In this study, 21 participants sat in a chair designed to simulate economy class seating in major airlines, and performed a range of foot and ankle exercises while in the seated position, followed by recommended airline activities undertaken hourly in accordance with airline recommendations, and then foot exercises alone, foot exercises against moderate resistance and foot exercises against increased resistance for 30 seconds every 15 to 20 minutes over a 100 minute period.

Sitting with feet flat on the floor resulted in a $40 \%$ decrease in popliteal blood flow velocity and a $42 \%$ decrease in blood flow volume at 100 minutes. The airline recommended activities started after 45 minutes of sitting (ankle rotation, heel-rise and toe-rise of the feet, knee flexion, forward back flexion and neck and shoulder rolls), resulted in a decreased blood flow velocity of $10 \%$ and decreased blood volume of $9 \%$ at 100 minutes; an improvement on sitting still, but still less than the baseline blood flow. The foot exercise alone comprised the heel-rise and toe-rise of the feet as recommended by airlines; this exercise resulted in a decreased velocity of $16 \%$ and decreased volume of $16 \%$ at 100 minutes. When the same exercise was assessed against moderate resistance (against a flat non-pivoting springboard), blood velocity decreased by $7 \%$, but volume increased by $2 \%$, 
and when was assessed against increased resistance (a pivoting pedal with an elastic tension band, which elicited maximal calf and foot muscle contraction) blood flow velocity increased by $15 \%$ compared to baseline and blood volume increased by $16 \%$ at 85 minutes, but was not sustained when the exercises were completed.

In this study Hito and colleagues provide evidence of improved popliteal blood flow while using specific devices when seated. However, this study was designed to replicate the venous haemodynamics of being seated on an aircraft, and may not be generalizable to a more ergonomic work environment where seat height and workstation height may be adjusted to meet the requirements of an individual user, and the commercial availability of equipment designed for workers to undertake leg and foot exercises while seated is limited. 


\section{The Legflow device}

The Legflow device (Medical Research Institute of New Zealand (MRINZ), Wellington, New Zealand) is a small, lightweight, plastic device with no mobile working parts that is designed to sit on the floor under the workstation of potential users. The Legflow device (Fig 1a) is approximately $260 \mathrm{~mm}$ in length and has two dome shapes on its upper surface that sit under the plantar venous plexus of each foot (or under the toes of each foot if users wear heeled shoes) allowing users to undertake the airline activities described above (heel-rise toe-rise) against a moderate resistance. In this instance however, the resistant part of the Legflow is dome shaped, allowing a larger and longer contraction of the foot and calf muscles compared to the flat non-pivoting board in the Hitos study. The domes are textured to allow users the choice to remove their footwear and take advantage of the massage effect if they so choose.

The Legflow device may be an unobtrusive, cost effective and practical tool that can help reduce venous stasis while seated and thus reduce the risk of VTE in a sedentary workers. Users flex and extend the sole of their foot slowly several times a minute over the dome, with the aim of increasing pressure on the plantar venous plexus and contracting the calf muscle. In this way, sedentary workers may be able to improve lower limb venous flow without the disruption and possible loss of productivity associated with workers needing to stand and moving around regularly.

To assess the efficacy of this device in improving venous flow in seated users, I completed a haemodynamic study of the popliteal blood flow in participants performing heel-rise and toe-rise exercises on the Legflow while seated. 


\section{STUDY FOUR: Legflow increases lower-limb venous haemodynamics when seated: a randomised controlled trial}

\section{Hypothesis}

The hypothesis of this study was that the Legflow device improves lower limb venous blood flow in adults who are seated.

\section{Primary outcome variable}

The primary outcome variable was the difference in peak systolic velocity (PSV) $(\mathrm{cm} / \mathrm{s})$, in the popliteal vein between the Legflow-mobilised and non-mobilised limb at 20 minutes

\section{Secondary outcome variable}

Secondary outcome variables were the difference in PSV between the Legflow-mobilised limb and non-mobilised limb after 15 minutes, and difference in mean flow velocity $(\mathrm{cm} / \mathrm{s})$, vein diameter $(\mathrm{mm})$, and total volume flow $(\mathrm{L} / \mathrm{min})$ between the Legflow-mobilised limb and the non-mobilised limb after 15 and 20 minutes

\section{Methods}

This was a randomised controlled trial of ten healthy adults, undertaken while they were sitting on an office chair. Popliteal vein flow was measured by compression ultrasound. One limb was randomised to be mobilised over the Legflow device at 15 and 20 minutes while the other limb remained immobile. The primary outcome variable was assessed by mixed linear model adjusted for baseline flow.

\section{Note}

This study has been reproduced with the permission of Occupational Ergonomics (I Braithwaite, Mackintosh, Mallon, Weatherall, \& Beasley, 2016). The introduction has been removed, and the discussion updated to reflect the updated literature review. Publisher approval for reproduction of this article can be found in Appendix XII. 


\section{Methods}

Ten adults aged between 18 and 65 years attended a screening visit at the offices of the Medical Research Institute of New Zealand at Wellington Regional Hospital for review of inclusion and exclusion criteria and informed consent (Appendix V). Potential participants were excluded if they had a history of previous or current deep vein thrombosis, were pregnant, or had a history of or clinical features of peripheral vascular disease, peripheral neuropathy, scleroderma, lymphoedema, or joint deformity from inflammatory arthritis. Participants that met all the inclusion and none of the exclusion criteria were shown how to use the Legflow device (Medical Research Institute of New Zealand (MRINZ), Wellington, New Zealand). Participants were instructed to repeatedly flex and extend the sole of the foot slowly over the dome device for approximately 60 seconds while two measurements were taken of venous popliteal flow using duplex ultrasonography. At the same time participants were asked to keep the other limb as still as possible.

A second visit was undertaken at Pacific Radiology, Lower Hutt, Wellington, for the study procedures. Each participant was seated on an adjustable office chair with both fleet flat on the floor and their thigh parallel to the ground. A goniometer was used to ensure that the angle between the thigh and the lower leg was 120 degrees. If necessary, small foam pads were placed under the feet to achieve and maintain this angle. Participants were seated forward enough on the chair that assessment of popliteal vein blood flow could be made by duplex ultrasonography without the participant having to move, this resulted in a 100 to $120 \mathrm{~mm}$ gap between the chair edge and the knee, not dissimilar to a usual office sitting position. Once positioning was achieved, participants were asked to sit quietly, without moving for 10 minutes at which point baseline measurements of popliteal venous flow were undertaken in both legs. The seated position is shown in Figure 4.1.

Imaging was done using a broadband linear ultrasound transducer (Philips IU22, Royal Philips, Eindhoven, The Netherlands) capable of both colour and pulsed Doppler. Auto calculation measurements were made over a 2 second interval. Cine loop review allowed for vein diameter measurement (used to calculate volume flow) with good temporal resolution. 
Figure 4.1: The top panel shows the Legflow Device, and the bottom panel shows the position of the participant during the study
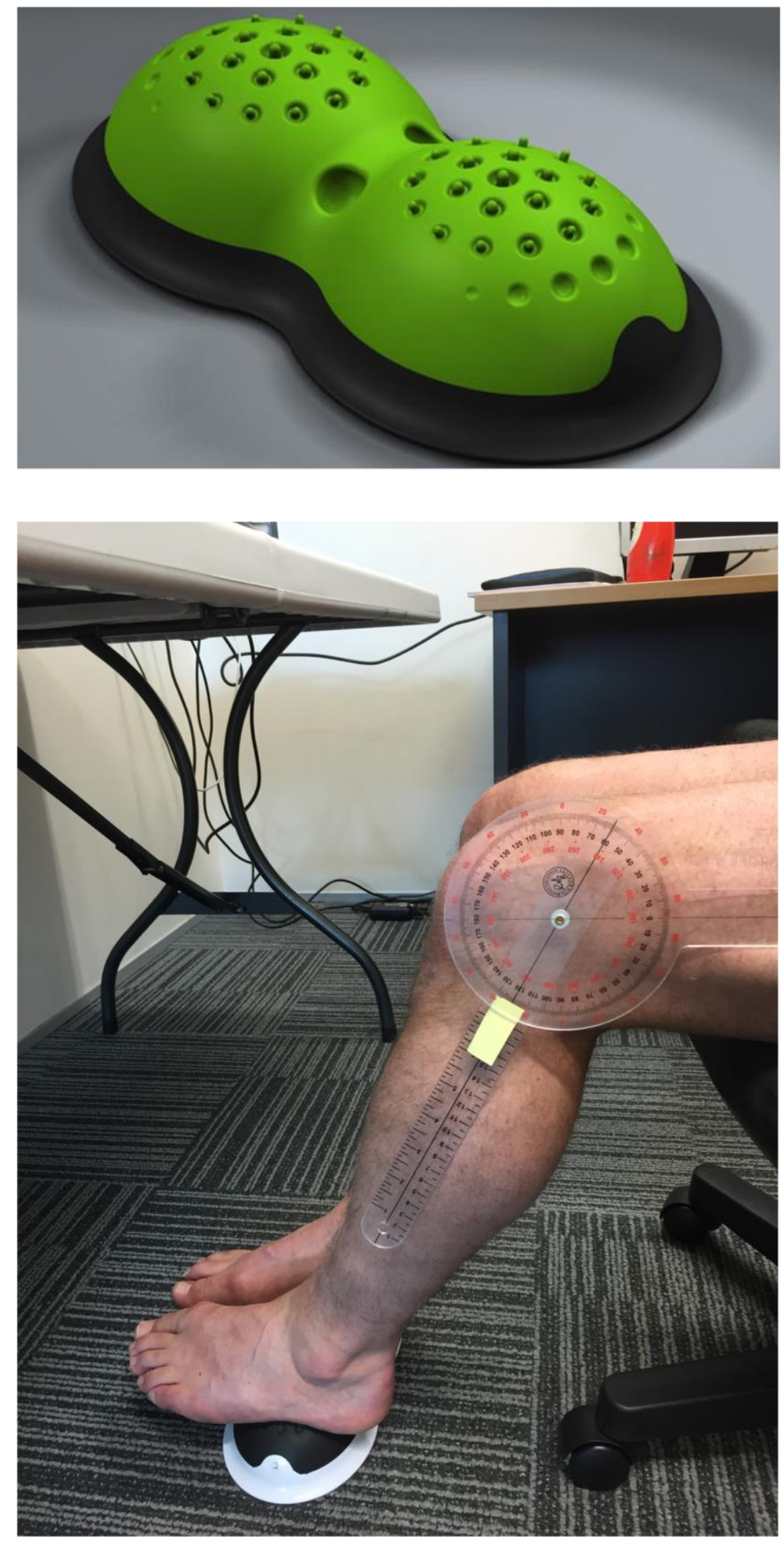
Participants were randomised to use the Legflow device with one leg at 15 minutes while keeping the other leg immobile (in its original position). Popliteal venous flow was measured in the Legflow-mobilised limb first, and then in the immobile limb. Once measurements were completed in the Legflow-mobilised limb, participants were asked to stop using the device with that limb. The same procedure was repeated at 20 minutes. After these measurements were undertaken, the study was deemed to be complete. All measures of popliteal vein flow at each time point were undertaken twice in each limb, and the mean of both measurements used.

The primary outcome variable was the difference in PSV $(\mathrm{cm} / \mathrm{s})$, in the popliteal vein between the Legflow-mobilised limb and the non-mobilised limb after 20 minutes. Secondary outcome variables were the difference in PSV between the Legflow-mobilised limb and non-mobilised limb after 15 minutes, and difference in mean flow velocity $(\mathrm{cm} / \mathrm{s})$, vein diameter $(\mathrm{mm})$, and total volume flow (L/min) between the Legflow-mobilised limb and the non-mobilised limb after 15 and 20 minutes.

\section{Statistical analysis}

Power calculations to determine an appropriate sample size were based on our previous study of the Micro-mobile ${ }^{\circledR}$ pneumatic foot compression device. In this study, device use was associated with a mean change from baseline popliteal PSV of $14.9 \mathrm{~cm} / \mathrm{s}$ (Charles et al., 2013) with a paired standard deviation of $7.49 \mathrm{~cm} / \mathrm{s}$ from the cross-over design for the change from baseline popliteal PSV. We calculated that a sample size of 10 participants had $90 \%$ power with a one-sided alpha of $5 \%$ to rule out a difference from baseline of less than $8 \mathrm{~cm} / \mathrm{s}$.

The primary outcome analysis was undertaken using a mixed linear model to estimate the overall difference between the two interventions, adjusting for baseline popliteal PSV. Secondary outcome variables were analysed using paired t-tests.

A post-hoc comparison was also made between the popliteal vein haemodynamics measured in the Legflow-mobilised limb and those reported in previous studies of mechanical and non-mechanical interventions used to promote venous flow while seated 
(Charles et al., 2013; Delis, Slimani, Hafez, \& Nicolaides, 2000; Hitos et al., 2007; Morita et al., 2006). Where we were unable to obtain the original data reported in the previous studies, data-points from the published graphics were independently measured by two investigators and in the case of initial disagreement, consensus was reached by negotiation.

Ethics approval was obtained from the Victoria University of Wellington Human Ethics Committee, approval number 20394, dated 08/04/2014 and the trial was prospectively registered with the Australia New Zealand Clinical Trials Registry: ANZCTR 12614000020684. 


\section{Results}

Five of the participants were women and the age range for all participants was 24 to 37 years. Participant BMI range was 22 to $31 \mathrm{~kg} / \mathrm{m}^{2}$. Mean popliteal vein blood-flow measurements assessed by duplex ultrasonography are shown in Table 4.1 and Figure 4.2. The mean (SD) PSV in the immobile limb was $5.21(1.45) \mathrm{cm} / \mathrm{s}$ at baseline and 4.41 (4.58) $\mathrm{cm} / \mathrm{s}$ and $4.58(1.09) \mathrm{cm} / \mathrm{s}$ after 15 and 20 minutes respectively. Mean (SD) PSV in the Legflow-mobilised limb was $5.82(1.59) \mathrm{cm} / \mathrm{s}$ at baseline and $55.3(18.3) \mathrm{cm} / \mathrm{s}$ and 63.3 (21.6) $\mathrm{cm} / \mathrm{s}$ after 15 and 20 minutes, representing a 9.5- and 10.9-fold increase from baseline.

In the Legflow-mobilised limb, mean (SD) flow velocity $(\mathrm{cm} / \mathrm{s})$ was increased 4.3 - and 4.2fold, and total flow volume (L/s) was increased 4.3- and 4.4-fold after 15 and 20 minutes compared to baseline while these parameters were unchanged in the immobile limb. Vein diameter was unchanged in both Legflow-mobilised and immobile limbs after 15 and 20 minutes compared to baseline. 
Table 4.1: Popliteal vein blood flow measurements as assessed by duplex ultrasonography.

\begin{tabular}{llll|lll}
\hline & \multicolumn{3}{c}{$\begin{array}{c}\text { Immobile limb } \\
\text { Mean (SD) }\end{array}$} & \multicolumn{3}{c}{$\begin{array}{c}\text { Legflow-mobilised limb } \\
\text { Mean (SD) }\end{array}$} \\
& Baseline & $\mathbf{1 5 \text { minutes }}$ & $\mathbf{2 0 \text { Minutes }}$ & Baseline & 15 minutes & 20 minutes \\
\hline $\begin{array}{l}\text { Peak systolic } \\
\text { velocity (cm/s) }\end{array}$ & $5.21(1.45)$ & $4.41(0.59)$ & $4.58(1.09)$ & $5.82(1.59)$ & $55.3(18.3)$ & $63.3(21.6)$ \\
$\begin{array}{l}\text { Mean flow } \\
\text { velocity (cm/s) }\end{array}$ & $1.69(1.00)$ & $1.39(0.46)$ & $1.41(0.82)$ & $2.01(0.95)$ & $8.74(1.94)$ & $8.48(3.67)$ \\
$\begin{array}{l}\text { Total volume } \\
\text { flow (L/min) }\end{array}$ & $0.068(0.038)$ & $0.060(0.022)$ & $0.066(0.044)$ & $0.079(0.048)$ & $0.345(0.133)$ & $0.346(0.021)$ \\
$\begin{array}{l}\text { Vein diameter } \\
\text { (mm) }\end{array}$ & $9.29(0.62)$ & $9.53(0.80)$ & $9.69(0.98)$ & $8.98(0.99)$ & $9.14(1.08)$ & $9.25(1.07)$ \\
\hline SD - standard deviation & & & & & \\
\hline
\end{tabular}

SD - standard deviation 
Figure 4.2. Popliteal vein blood flow measurements in the immobile and Legflow mobilised legs at baseline, $\mathbf{1 5}$ and $\mathbf{2 0}$ minutes as assessed by duplex ultrasonography. Clockwise from top left panel: Peak Systolic Velocity, Mean Flow Velocity, Vein Diameter and Total Volume Flow.
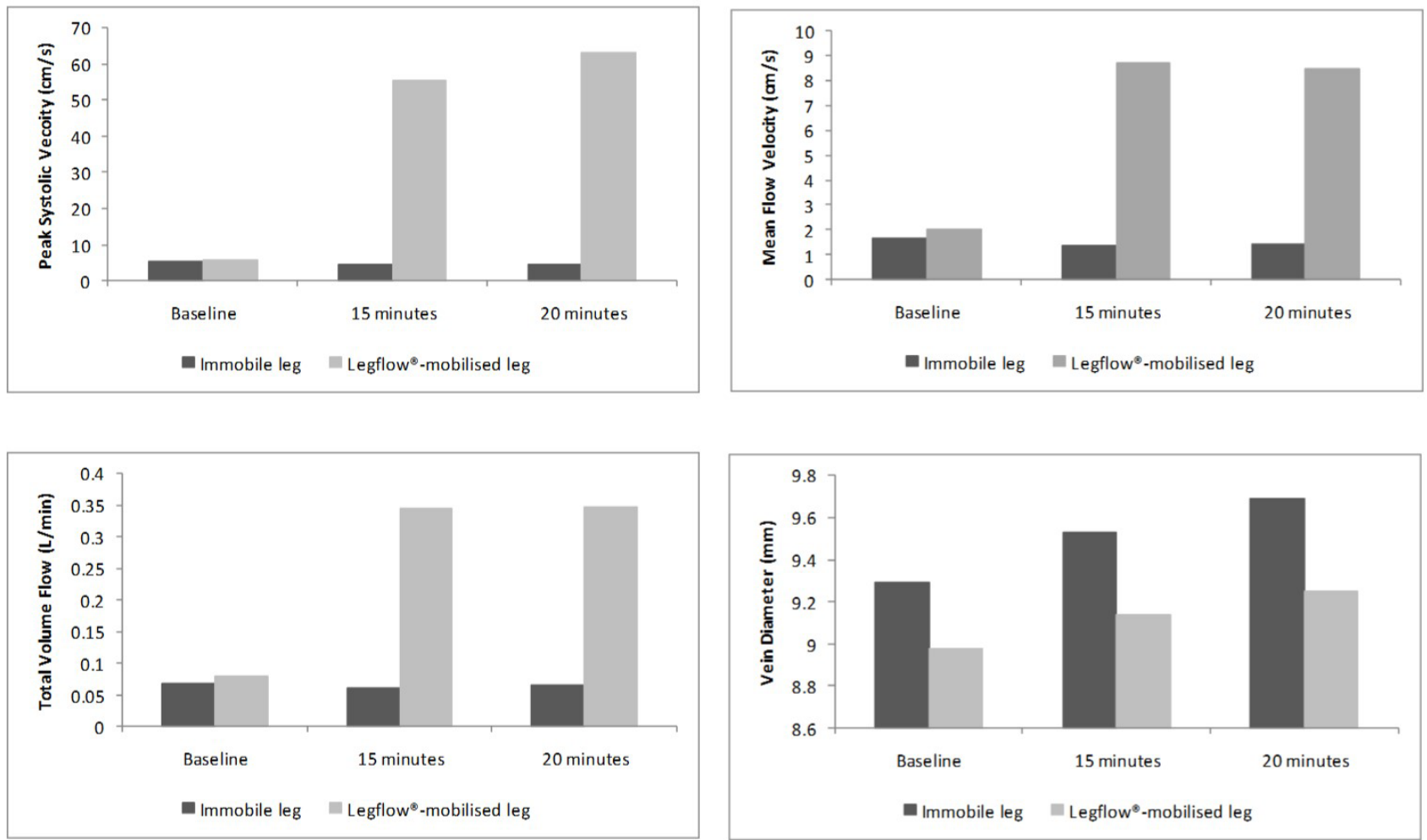
The differences in popliteal vein blood flow between the Legflow-mobilised limb and the immobile limb are shown in Table 4.2 and Figure 4.3. After 20 minutes, the mean (SD) PSV achieved in the Legflow-mobilised limb was $58.8(21.5) \mathrm{cm} / \mathrm{s}$ greater than the immobile limb. The mean (SD) change in PSV from baseline in the Legflow-mobilised limb was 58.2 $(22.1) \mathrm{cm} / \mathrm{s}$ greater than the immobile limb. The estimate $(95 \% \mathrm{Cl})$ of the true difference in PSV between the Legflow-mobilised limb and the immobile limb adjusted for baseline measurements was $60.0 \mathrm{~cm} / \mathrm{s}(95 \% \mathrm{Cl} 44.6$ to 75.3$), \mathrm{P}<0.001$.

The difference in mean flow velocity $(\mathrm{cm} / \mathrm{s})$ and total volume flow $(\mathrm{L} / \mathrm{min})$ between the Legflow-mobilised limb and the immobile limb adjusted for baseline was also statistically significant, while the vein diameter $(\mathrm{mm})$ did not change. 
Table 4.2. Differences in popliteal vein blood flow between Legflow-mobilised limb and immobile limb at each time point.

\begin{tabular}{llll}
\hline & $\begin{array}{l}\text { Legflow minus } \\
\text { immobile } \\
\text { Mean (SD) }\end{array}$ & $\begin{array}{l}\text { Change from baseline - } \\
\text { Legflow minus immobile } \\
\text { Mean (SD) }\end{array}$ & $\begin{array}{l}\text { Estimate (95\%Cl) of difference } \\
\text { Legflow minus immobile, } \\
\text { P value }\end{array}$ \\
\hline $\begin{array}{l}\text { Peak systolic } \\
\text { velocity (cm/s) }\end{array}$ & $58.8(21.5)$ & $58.2(22.1)$ & \\
$\begin{array}{l}\text { Mean flow } \\
\text { velocity (cm/s) }\end{array}$ & $7.1(3.9)$ & $6.8(3.9)$ & $7.1(4.6$ to 9.6), <0.001 \\
$\begin{array}{l}\text { Total volume } \\
\text { flow (L/min) }\end{array}$ & $0.280(0.205)$ & $0.269(0.214)$ & $0.278(0.127$ to 0.428$),<0.001$ \\
$\begin{array}{l}\text { Vein diameter } \\
\text { (mm) }\end{array}$ & $-0.45(1.09)$ & $-0.13(0.99)$ & $-0.24(-0.93$ to 0.46$), 0.46$ \\
\hline
\end{tabular}

15 minutes

\begin{tabular}{llll}
\hline $\begin{array}{l}\text { Peak systolic } \\
\text { velocity }(\mathrm{cm} / \mathrm{s})\end{array}$ & $50.9(18.0)$ & $50.3(18.8)$ & $50.8(37.1$ to 64.6$),<0.001$ \\
$\begin{array}{l}\text { Mean flow } \\
\text { velocity (cm/s) }\end{array}$ & $7.1(1.6)$ & $6.8(1.6)$ & $6.8(5.7$ to 8.0$),<0.001$ \\
$\begin{array}{l}\text { Total volume } \\
\text { flow }(\mathrm{L} / \mathrm{min})\end{array}$ & $0.249(0.121)$ & $0.273(0.119)$ & $0.268(0.194$ to 0.341$),<0.001$ \\
$\begin{array}{l}\text { Vein diameter } \\
\text { (mm) }\end{array}$ & $-0.39(1.13)$ & $-0.08(0.64)$ & $-0.09(-0.56$ to 0.38$), 0.67$ \\
\hline $\begin{array}{l}\text { SD - Standard deviation } \\
\mathrm{Cl}-\text { Confidence interval }\end{array}$ & & \\
\hline
\end{tabular}


Figure 4.3. Popliteal vein blood flow: change from baseline (Legflow mobilised limb minus immobile limb) at $\mathbf{1 5}$ and $\mathbf{2 0}$ minutes as assessed by duplex ultrasonography. The horizontal lines are the 25th, median, and 75th percentiles, symbols are the mean and the whiskers are from minimum to maximum. Clockwise from top left panel: Peak Systolic Velocity, Mean Flow Velocity, Vein Diameter and Total Volume Flow.
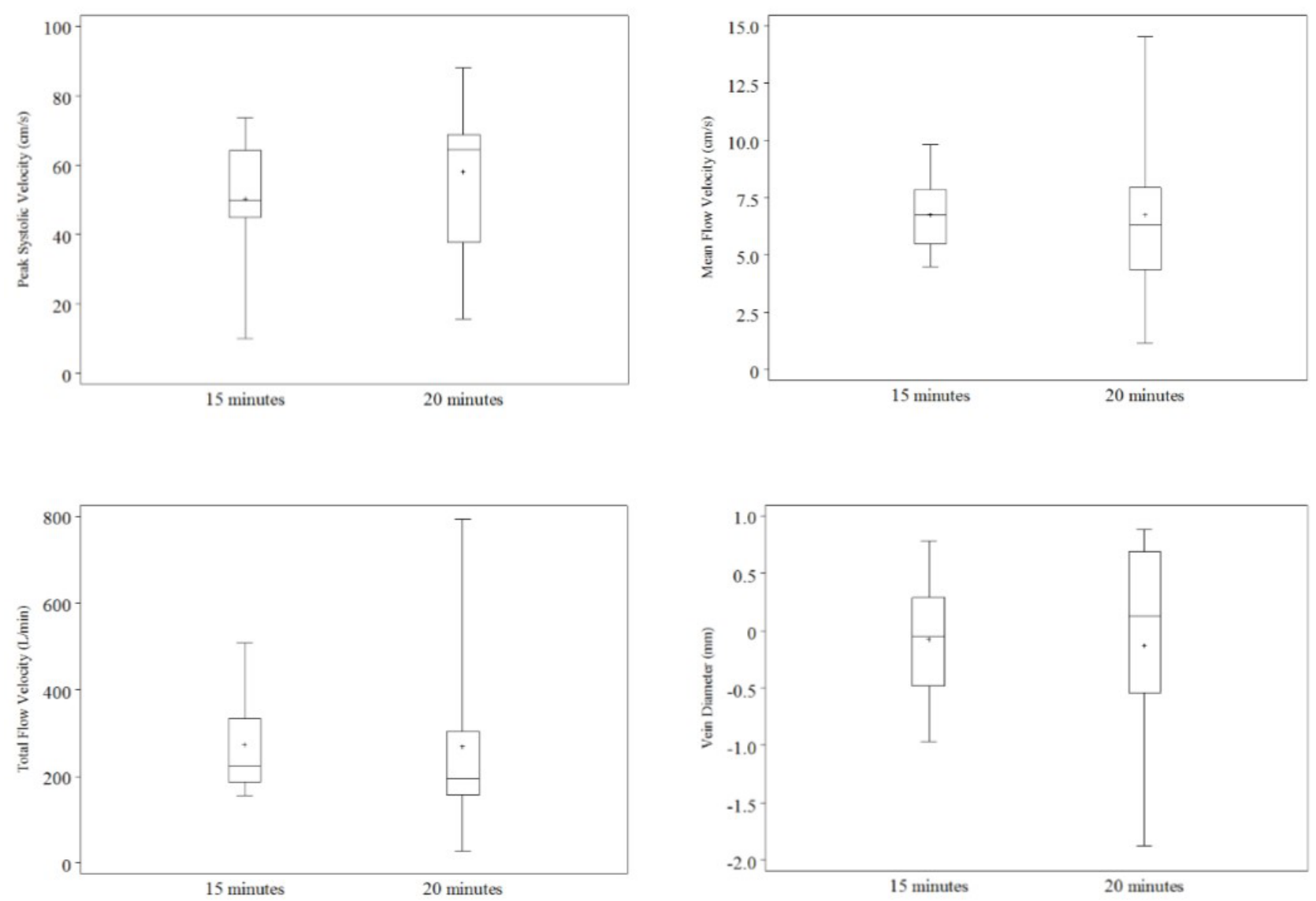
Table 4.3 shows popliteal vein haemodynamics of seated participants using the Legflow device compared to that reported in other studies using other mechanical and nonmechanical interventions to promote venous flow while seated. The haemodynamic measurements at baseline were comparable with baseline measurements of seated participants in the identified studies (Charles et al., 2013; Delis et al., 2000; Hitos et al., 2007; Morita et al., 2006).

The mean PSV $(63.3 \mathrm{~cm} / \mathrm{s})$ achieved in this Legflow device study was higher than that of the micro-mobile foot pneumatic compression device (Charles et al., 2013) and exceeded the PSV achieved with neuromuscular electrical stimulation (NMES) of the gastrocnemius, a foot IPC with inflation pressure of $120 \mathrm{mmHg}$ and a foot IPC with inflation pressure of $180 \mathrm{mmHg}$ (Delis et al., 2000). The mean PSV was lower than IPC of the calf and IPC of the foot and calf combined (Delis et al., 2000).

The mean flow velocity achieved with the Legflow was greater than that of the Micromobile compression device and NMES of the gastrocnemius. It was similar to that achieved with recommended airline activities, foot exercises, foot exercises against resistance and foot exercises against increased resistance, but was less than that achieved with any IPC device. 
Table 4.3: Comparison of popliteal venous haemodyamics measured in the Legflow study compared with other studies measuring the effects of mechanical and non-mechanical interventions in seated participants.

\begin{tabular}{|c|c|c|}
\hline Device & $\begin{array}{l}\text { PSV }(\mathrm{cm} / \mathrm{s}) \\
\text { Mean (SD) }\end{array}$ & $\begin{array}{c}\text { Mean flow velocity }(\mathrm{cm} / \mathrm{s}) \\
\text { Mean (SD) }\end{array}$ \\
\hline Legflow device & $63.3(21.6)$ & $8.48(3.67)$ \\
\hline \multicolumn{3}{|c|}{ Intermittent pneumatic compression devices } \\
\hline $\begin{array}{l}\text { Micro-mobile }{ }^{\circledR} \text { compression device (Charles et al., } \\
\text { 2013) }\end{array}$ & $20.1(6.77)$ & $5.78(1.85)$ \\
\hline $\begin{array}{l}\text { Arterio-Venous Impulse system }{ }^{\circledR} \text { (Novamedix) } \\
120 \mathrm{~mm} / \mathrm{Hg} \text { (foot)(Delis et al., 2000) }\end{array}$ & 42.0 & 15.0 \\
\hline $\begin{array}{l}\text { Arterio-Venous Impulse system }{ }^{\circledast} \text { (Novamedix) } \\
180 \mathrm{~mm} / \mathrm{Hg} \text { (foot)(Delis et al., 2000) }\end{array}$ & 53.0 & 18.5 \\
\hline $\begin{array}{l}\text { Art-Assist }{ }^{\circledR} \text { Model AA-1000 unit } \\
120 \mathrm{~mm} / \mathrm{Hg} \text { (calf)(Delis et al., 2000) }\end{array}$ & 115.0 & 28.5 \\
\hline $\begin{array}{l}\text { Art-Assist }^{\circledR} \text { Model AA-1000 unit } \\
120 \mathrm{~mm} / \mathrm{Hg} \text { (foot and calf)(Delis et al., 2000) }\end{array}$ & 146.0 & 35.5 \\
\hline
\end{tabular}

\section{Non-mechanical seated exercises}

\begin{tabular}{ll}
\hline Airline recommended activities(Hitos et al., 2007)* & 9.8 to 19.4 \\
Foot exercises(Hitos et al., 2007)** & 9.5 to 20.2 \\
$\begin{array}{l}\text { Foot exercises against moderate resistance(Hitos et } \\
\text { al., 2007)*** }\end{array}$ & 7.3 to 13.4 \\
$\begin{array}{l}\text { Foot exercises against increased resistance(Hitos et } \\
\text { al., 2007)**** }\end{array}$ & 10.1 to 18.1
\end{tabular}

Neuromuscular Electrical Stimulation

NMES to gastrocnemius (averaged over 4 time $\quad 17.07$

points)(Morita et al., 2006)

SD's were not available for all published studies

- $\quad$ ankle rotation (clockwise or anticlockwise), heel-raise and toe-raise, knee flexions, forward -back flexion, neck and shoulder roll

** heel-raise and toe-raise

*** active plantar- and dorsiflexion performed against a flat, non-pivoting springboard

$* * * *$ active plantar- and dorsiflexion on pivoting board attached to an elastic tension band

PSV - Peak systolic velocity

SD - Standard deviation 


\section{Discussion}

Use of the Legflow resulted in a marked increase in systolic popliteal venous flow with a 10.9-fold greater PSV and a 4.4-fold greater total volume flow compared to the immobile limb. These haemodynamic effects are likely to be due to both contraction of the calf muscles and pressure on the plantar venous plexus. These findings indicate that its use would not only prevent lower limb venous stasis secondary to immobility, but also enhance lower limb venous haemodynamics, effects which have the potential to reduce VTE risk. The simplicity of the device, the improvement in venous haemodynamics and its compact size would make it a cost-effective intervention to reduce venous stasis associated with prolonged seated immobility.

There are a number of methodological issues relevant to the study findings. The participants' non-mobilised limb acted as a control against which to compare the effects of Legflow-mobilisation. To mitigate the inability to blind participants or investigators to the intervention, the Legflow-mobilised limb was selected according to a randomisation schedule generated by a statistician ensuring there was no potential bias accrued from investigator selection of the left or right leg. The study was adequately powered to detect a clinically relevant change in blood flow. The study was undertaken in healthy young adults with no clinical evidence of peripheral neuropathy or vasculopathy, and so the results may not be generalisable to an older population with comorbidities or other potential users of the device who did not meet the study inclusion and exclusion criteria.

To assess the potential utility an effectiveness of the Legflow it is informative to compare its haemodynamic effects with other devices used to improve venous haemodynamics and/or reduce VTE risk. Two important comparisons are with airline recommended exercises to promote venous flow while undertaking long distance flights and with foot and calf IPC devices which have been shown to reduce the risk of VTE in at risk situations associated with surgery and are widely used for this indication. The Legflow is more effective at increasing popliteal vein PSV in the seated position than an IPC applying pressure to the plantar venous plexus (Delis et al., 2000) and to isolated NMES of the gastrocnemius (Morita 
et al., 2006), but is not as effective as IPC to the calf and foot, or IPC the calf alone (Delis et al., 2000).

The mean flow velocity seen with the Legflow is in keeping with that achieved while undertaking airline recommended exercises and foot exercises with and without resistance (Hitos et al., 2007). While individuals may achieve a similarly increased mean flow velocity by voluntarily undertaking active exercises without the use of a device, it is possible that the likelihood of this being done would be increased by the presence of the Legflow acting as a visual reminder to users. The higher mean flow velocity seen in IPC devices (Delis et al., 2000) compared with the airline exercises (Hitos et al., 2007) and the Legflow-mobilisation is likely to be due to the gradual reduction in pressure on the plantar venous plexus and calf veins with the IPC devices, rather than the rapid relaxation and decompression that is more likely to occur in active physical exercise. However, use of IPC devices in the workplace or during long distance travel is both impractical and costly.

\section{Conclusion.}

This study has shown that for a healthy population, Legflow-mobilisation of the lower limb while seated results in significantly increased venous flow compared to non-mobilisation, and that this was at least as effective as IPC to the feet and airline recommended exercises both with and without resistance. Having demonstrated the beneficial effect on the haemodynamics of the popliteal vein in those seated in office chairs, the next step is to assess the feasibility of the Legflow device in the sedentary workplace by assessing utilisation of the device in that working environment. 


\section{Chapter Five: Interventions for the prevention of venous thromboembolism in the orthopaedic setting}

Lower limb immobilisation, by contrast with sedentary office work, is not a directly modifiable risk factor for VTE. Instead interventions need to be focused reducing the risk of VTE during the period of immobilisation. Since the 1930 s attempts have been made to reduce the risk of PE after trauma and surgery. In the absence of pharmacological agents in the 1930s, Homans described the process of femoral ligation to prevent any DVT from embolising to the lungs (Homans, 1934).

The two main modern-day approaches to reduce risk of VTE in the orthopaedic setting are pharmacological interruption of the coagulation cascade using medications such as low molecular weight heparin (LMWH), warfarin, rivaroxaban, and aspirin, and mitigation of venous stasis using early mobilisation strategies, graduated compression stocking of varying lengths, and / or intermittent pneumatic compression (IPC) devices involving full leg length compression, thigh compression, calf or foot compression or combinations of any of these.

There is no shortage of medical literature about interventions for reducing the risk of VTE in the orthopaedic setting, but somewhat surprisingly, VTE prophylaxis in lower limb immobilisation is an area of orthopaedics in which there is relatively little evidence, and opinion is divided. 


\section{Low molecular weight heparin}

Low Molecular Weight Heparins (LMWHs) were manufactured in the 1980s and remain a mainstay of prophylaxis and treatment for VTE events today. LMWH is a collection of short chain polysaccharide heparin salts, with an average molecular weight of $8000 \mathrm{Da}$ and is derived from unfractionated heparin. The main effect of LMWHs on the coagulation cascade is to bind to antithrombin forcing a conformational change in in this molecule, thereby accelerating inhibition of Factor Xa.

Prophylactic doses of LMWH are fixed and can be given subcutaneously once daily, making prolonged outpatient treatment possible. Heparin induced thrombocytopenia is less common than with the use of heparin alone and routine laboratory monitoring is not usually required. Patients can be shown how to inject themselves with LMWH which may reduce the burden on health service delivery systems but may be a barrier to prolonged treatment.

\section{Low molecular weight heparin in orthopaedic surgery}

There are many studies in the medical literature outlining the effect of LMWH in major orthopaedic surgery. In one of the first, Turpie and colleagues reported a RCT of fixed dose LMWH versus placebo for the prevention of VTE in elective hip surgery (Turpie et al., 1986). 100 patients were randomised to either 30mg of LMWH twice daily or placebo 12 to 24 hours after surgery and treatment was continued for 14 days or until discharge. Initially investigators planned to undertake venography for diagnosis of DVT only if screening tests were negative. However, rates were very low in the first 24 patients, so the remaining 76 were planned to undergo venography regardless of the outcome of surveillance tests. In those 76 patients, 4 in the LMWH group were found to have VTE versus $20 \%$ in the placebo group. In the entire group of 100, 12 in the LMWH groups had VTE and $42 \%$ in the placebo group had VTE. Bleeding complications were the same between the two groups.

Planes and colleagues then compared LMWH to unfractionated heparin, finding that a once daily dose of enoxaparin started 12 hours pre-operatively was superior to unfractionated heparin administered intravenously three times daily in a group of 237 patients undergoing total hip replacement (Planes et al., 1988). Treatment was continued for 15 days or until 
discharge. Proximal DVT rates found after venography were $18.5 \%$ in the heparin group compared to $7.5 \%$ in the LMWH group, and DVT rates were $25 \%$ and $12.5 \%$ respectively. The LMWH groups required fewer blood transfusions, but did have two major bleeds and one minor bleed compared to two minor bleeds in the heparin group.

\section{The argument for extended prophylaxis with low molecular weight heparin}

By 1993, the body of evidence was favouring LMWH over placebo, dextran or unfractionated heparin as a prophylactic agent for the prevention of VTE in both general and orthopaedic surgery (L. N. Jorgensen et al., 1993). While LMWH was being used increasingly for surgical inpatients until discharge, there was a still concern over postdischarge VTE rate. In 1996 Planes carried out a single centre, prospective, double blind RCT to quantify the risk of VTE after total hip replacement. 179 patients who had undergone total hip replacement and who had no DVT on bilateral ascending venography at discharge were recruited. 89 participants were randomised to placebo and 90 participants to $40 \mathrm{mg}$ enoxaparin daily. All patients were considered low risk for VTE at baseline, had been receiving LMWH while in hospital and had been advised to wear compression stockings at discharge. Venography was repeated 21 days after discharge or earlier if there were symptoms of VTE. The rate of DVT was $7.1 \%$ and $19.3 \%$ in the enoxaparin and placebo groups respectively with three minor bleeds in the enoxaparin group and one in the placebo group.

In the same year Bergqvist and colleagues undertook a RCT of LMWH (enoxaparin) as prophylaxis for VTE after total hip replacement (Bergqvist et al., 1996). All patients received enoxaparin in hospital and were then randomised to receive either aspirin or LMWH at discharge. Bilateral ascending phlebography was performed 19 to 23 days after discharge. Of the participants where adequate venography was achieved, there were 43 DVTs and 2 PEs in the placebo group and 21 DVTs and no PEs in the enoxaparin group. There was no difference in the rates of haematoma formation between treatments.

In 2001, after a review of RCTs assessing prolonged prophylaxis after lower limb surgery, Cohen and colleagues concluded that extended prophylaxis with LMWH would result in a $50 \%$ reduction in the development of both symptomatic and clinical (diagnosed through 
screening) VTE events (A. T. Cohen, Bailey, Alikhan, \& Cooper, 2001). By 2008, guidelines were recommending routine use of LMWH peri-operatively for all major surgery unless contraindicated, and a duration of treatment of a minimum of ten days and up to 35 days in the case of hip joint replacement, knee joint replacement and hip fracture surgery (Geerts et al., 2008).

\section{Low molecular weight heparin in lower limb immobilisation}

The role of LMWH in the context of lower limb immobilisation has also been demonstrated, and a body of six RCTs have formed the basis of a series of Cochrane reviews of prophylaxis in this clinical setting (Ettema et al., 2008; Mark Testroote et al., 2014).

In 1993 Kujath and colleagues enrolled 253 patients, randomising 126 to receive a LMWH (Fraxiparin) daily for the duration of the lower limb immobilisation and 127 to receive no prophylaxis (Kujath et al., 1993). 30\% of patients had bone injuries and $70 \%$ had soft tissue injuries. Compression sonography was carried out on the day the cast was removed or sooner in the event of clinical symptoms, and where there was doubt, phlebography was used to confirm VTE diagnosis. $4.8 \%$ of patients on LMWH were diagnosed with VTE compared to $16.5 \%$ in the untreated group. In the soft tissue injuries DVT was diagnosed in $2.4 \%$ and $11.3 \%$ of the LMWH and untreated groups respectively and in the case of bony injuries DVT was diagnosed in $10.3 \%$ and $29 \%$ of the LMWH and untreated groups respectively. One third of all DVTs were symptomatic and $11 \%$ were proximal. With respect to pre-existing risk factors for VTE, in both groups, the patients that developed DVT were more likely to be overweight. According to the investigators, those that did not develop VTE had 1.24 risk factors and those that did develop VTE had 1.96 risk factors.

The Kock trial in 1995 reported findings of an open label RCT in which 428 patients with leg injuries conservatively treated in the community with lower limb immobilisation, were randomised to either $\mathrm{LMWH}$ or no prophylaxis for the duration of the immobilisation (Kock et al. 1995). Above knee plasters were applied to $14 \%$ of patients in each group, the rest were below the knee. Just over one third in each group had Grade II sprains which were immobilised for an average of seven days, and a similar number had grade III sprains which had an average immobilisation of around three weeks. Most of the rest were fractures, 
immobilised for about three weeks in the LMWH group and just over four weeks in the control group. Randomisation was stratified by varicose veins and obesity. VTE was diagnosed by a combination of clinical examination, venous occlusion plethysmography and compression ultrasound, with confirmatory phlebography. The study was terminated at the third interim analysis, because in the 163 patients on no treatment there were seven DVTs compared to none in the LMWH group, and this fell below the critical significance level for termination in the protocol. While there was a significant difference in the duration of lower limb immobilisation between the two groups (15.2 days in the LMWH group and 18.8 days in the untreated group), a correlation between days immobilised and VTE could not be demonstrated. Kock concluded that this was further evidence for the use of LMWH in the prevention of VTE in lower limb immobilisation.

In 2002 Jorgensen and colleagues undertook an assessor blinded RCT of 300 patients with planned plaster cast lower limb immobilisation of a minimum of three weeks (Jorgensen et al. 2002). Three quarters of the patients had suffered fractures and $20 \%$ had suffered Achilles tendon ruptures. Patients were randomised to a LMWH (Innohep) daily for the duration of their lower limb immobilisation or to no prophylaxis. 205 participants underwent ascending unilateral venography of the previously immobilised limb on the day their cast was removed. In the LMWH group 10/99 patients were diagnosed with clinical DVT, as were $18 / 106$ patients in the treatment group. There were no symptomatic DVTs, and only one was proximal (non-treatment group). While the odds ratio for association for VTE in the treatment groups was half that in the treatment group, the difference was not statistically significant. In the Innohep group roughly the same proportion of tendon ruptures and fractures were diagnosed with VTE (10\% and $11 \%$ respectively). In the untreated group, $30 \%$ of tendon ruptures and $13 \%$ of the fractures were diagnosed with DVT. Jorgensen concluded that while 10 to $20 \%$ of patients with lower limb immobilisation would develop clinical DVTs, Innohep was not an effective preventive treatment in this clinical setting.

In the same year Lassen and colleagues reported the results of a RCT in which 440 patients who required immobilisation of the lower limb in a plaster cast or brace for a minimum of 
five weeks were randomised to receive either LMWH (Reviparin) or placebo (M. R. Lassen et al., 2002). Patients who underwent surgery prior to lower limb immobilisation ( $55 \%$ in this study) were allowed up to four days of heparin prophylaxis prior to randomisation, and as a result, approximately one third of the patients in each group had received heparin prior to randomisation. In the LMWH group 24\% had an Achilles tendon injury, and the remainder had suffered fractures. In the placebo group $16 \%$ had an Achilles tendon injury and the rest had suffered fractures. All patients were able to mobilise on crutches from randomisation. Treatment was given for the period of immobilisation and venography of the immobilised limb was performed within one week of removal of the cast or brace or earlier if symptoms suggested the presence of a DVT. 371 patients completed the study. $9 \%$ of the LMWH groups were diagnosed with DVT compared to $19 \%$ in the placebo group. In the LMWH group, three patients had a proximal DVT compared to ten in the placebo group (of which two had PEs) but this was not statistically significant. There were no symptomatic DVTs in the LMWH group, compared to two symptomatic DVTs in the placebo group. There was no difference between those who had previously received heparin and those who did not. The comparative effect of LMWH compared to placebo was similar in both the fracture and the Achilles tendon patients. Lassen concluded that LMWH was effective in reducing VTE rates in the setting of lower limb immobilisation.

Five years later Lapidus and colleagues reported two clinical trials. The first was a prospective placebo controlled trial of LMWH (dalteparin) or placebo in 105 patients who underwent surgery for Achilles tendon rupture, with the treatments to be administered for six weeks (L. J. Lapidus, Rosfors, et al., 2007). In standard management, the patients were non weight-bearing for the first three weeks and then progressed to full weight-bearing in the following three week period. All patients were screened for DVT at the end of the study with sonography, and any diagnosis of DVT was confirmed with phlebography. 91 participants were analysed at the end of the study. $34 \%$ of the LMWH group and $36 \%$ of the placebo group were diagnosed with DVT. Proximal DVT occurred in one LMWH patient and three placebo patients. The high proportion of DVT in this patient group is notable, and the apparent lack of effectiveness of dalteparin in reducing the rate is disappointing. Lapidus hypothesised that this lack of effectiveness might be due to the placement of the foot in 
equinus potion in the first three weeks of immobilisation, making weight-bearing on the injured limb impossible, and thus participants would be completely unable to activate the venous plantar and calf pumps that would normally be activated with walking.

The second Lapidus trial was a RCT comparing treatment with dalteparin to placebo for a five week period for the prevention of VTE in patients undergoing ankle fracture surgery. All patients received dalteparin for one week before randomisation. The incidence of DVT in the LMWH group was $21 \%$ compared to $28 \%$ in the placebo group and the incidence of proximal DVT was $4 \%$ and $3 \%$ in the LMWH and placebo groups respectively. There was no difference between one week of treatment with dalteparin and five weeks treatment.

\section{Guidelines regarding lower limb immobilisation and prophylaxis for venous thromboembolism}

The six trials covered in the Cochrane analysis used a total of five different types of LMWH, and included patients with a range of conditions including soft tissue injuries or fractures, with variable periods of immobilisation, treated conservatively and surgically, with above or below knee casts and varying degrees of weight-bearing capacity, which makes direct comparison of results challenging. All the studies used screening strategies for the detection of asymptomatic DVTs. Few DVTs were symptomatic and the proportion that was proximal was low.

Regardless, the Cochrane review of 2008 conducted a meta-analysis of these six trials and concluded that VTE rates of $4.3 \%$ to $40 \%$ in the placebo patients were significantly higher than those in the LMWH group ( $0 \%$ to $37 \%$ ) and the odds ratio for association with VTE in the LMWH group was 0.49 times that of the placebo group (M Testroote, Stigter, de Visser, \& Janzing, 2008). This was the case whether patients were surgically treated or conservatively managed or had fractures or soft tissue injuries. Therefore, the overall recommendation was to prescribe LMWH in the clinical setting of lower immobilisation.

In stark contrast, in June 2008 the American College of Chest Physicians (ACCP) recommended no routine prophylaxis for patients with isolated lower limb injuries distal to the knee (Geerts et al., 2008) with little further discussion. The ACCP did cover this in some 
detail in their 2004 guidelines for the prevention of thromboembolism, citing an incidence of asymptomatic DVT in patients with below knee injuries of 10 to $40 \%$, but stating they did not recommend prophylaxis as they were not convinced that the use of LMWH prophylaxis would reduce the incidence of 'clinically important VTE' nor that LMWH was cost-effective (Geerts et al., 2004). This highlights the disagreement over the clinical importance of the predominantly distal and asymptomatic DVTs seen in the medical literature at that time. By 2012, the ACCP had changed their stance somewhat, indicating that they were still unconvinced about the benefit of thromboprophylaxis demonstrated in the Cochrane study, but also stated that further research was required in this area, particularly with respect to 'anticoagulant thromboprophylaxis versus aspirin stratified by type of injury and procedure and expected degree of immobility' (Falck-Ytter et al., 2012).

The National Institute of Health and Care Excellence (NICE) UK guidelines recommend assessing patients for VTE risk, and for those in lower limb casts who are considered high risk (have at least one other risk factor for VTE), 'considering' LMWH as prophylaxis (National Institute for Health and Clinical Excellence 2010).

In the Guidelines in the Emergency Medicine Network (GEMnet) publication with respect to the ambulatory trauma patients with lower limb immobilisation, it was agreed that there was reasonable evidence to suggest a significant risk of VTE in patients with isolated lower limb injury and subsequent lower limb immobilisation (Roberts et al., 2013), and that there was no validated prediction score to allow assessment of VTE risk in this setting. They recommended that ambulatory patients with lower limb injuries and rigid immobilisation, non-weight-bearing status, acute severe injuries or any other risk factor for VTE should be considered to be at increased risk of VTE and consideration should be given to routine prophylaxis with LMWH.

The Italian intersociety consensus statement on antithrombotic prophylaxis in orthopaedics and traumatology (Randelli et al., 2013) suggested LMWH for VTE prophylaxis in lower limb immobilisation or non-weight-bearing situations, at least until the patient is mobilised and ankle mobility and weight-bearing capacity are 'partially' restored. 
Current New Zealand practice is not to routinely use LMWH for VTE prophylaxis in the clinical setting of lower limb immobilisation. Regardless of any disagreement over the risk and/ or risk stratification in this group of patients, the clinical significance of asymptomatic and distal DVTs and the incidence of clinically significant VTE in this diverse patient group, LMWH is not funded by the government funding authorities in New Zealand for the primary prevention of VTE in this clinical setting.

Future randomised controlled trials of low molecular weight heparin for the prevention of venous thromboembolism in lower limb immobilisation

A review of the clinical trials.gov website shows one RCT of LMWH versus no LMWH in lower limb immobilisation currently underway (S. Cannegieter, 2012). Started in 2012, with estimated completion date for collection of the primary outcome variable in June 2016 and run by Dr S Cannegeiter in collaboration with the Netherlands organisation for Health Research and Development, this is a study of 1,500 patients undergoing lower limb immobilisation, half of which are randomised to LMWH and the other half to no LMWH. The primary end point is symptomatic DVT at three months. Each hospital involved in the study will prescribe $\mathrm{LMWH}$ to those randomised to this intervention according to their own guidelines and / or physician preference. The size of the study is substantial. While there is no discussion about the power calculations of the study, the investigators have made it clear that the intention of such a large study is to overcome the apparent ambiguity of the risks and benefits demonstrated in smaller preceding studies. 


\section{Rivaroxaban}

Rivaroxaban is an oral anticoagulant that directly inhibits Factor Xa, the dose of which does not change for sex, age or BMI, but does need modification in the setting of renal impairment (Bayer, 2015). Monitoring of plasma levels is not required.

\section{Rivaroxaban: extended prophylaxis in total hip arthroplasty}

In the REgulation of Coagulation in major Orthopaedic surgery Reducing risk of DVT and pulmonary embolism (RECORD) studies, Rivaroxaban was tested against LMWH in a range of clinical settings. RECORD 1 was a RCT compared the efficacy and safety of rivaroxaban with enoxaparin for extended VTE prophylaxis in those undergoing total hip arthroplasty (B. Eriksson et al., 2008). 4541 patients were randomised to receive either 10mg rivaroxaban orally or 40mg of enoxaparin subcutaneously once a day for 35 days. 3,153 patients were included in the final efficacy analysis which comprised a composite primary outcome of DVT (symptomatic or detected by mandatory bilateral venography), non-fatal PE or death from any cause at the end of the study. $1.1 \%$ in the rivaroxaban group and $3.7 \%$ in the LMWH group met the primary efficacy outcome. Major VTE (a composite of proximal DVT, nonfatal $\mathrm{PE}$, or death from VTE), occurred in $0.2 \%$ in the rivaroxaban group and $2.0 \%$ of the $\mathrm{LMWH}$ group. There was no difference in the major bleeding rates (defined as bleeding that was fatal, occurred in a critical organ (e.g., retroperitoneal, intracranial, intraocular, and intraspinal bleeding) or required reoperation or extrasurgical-site bleeding that was clinically overt and was associated with a fall in the haemoglobin level of at least $20 \mathrm{~g} / \mathrm{L}$ or that required transfusion of 2 or more units of whole blood or packed cells), between the two groups. In this analysis, Rivaroxaban appears a safe and effective treatment compared to LMWH for the long term prevention of VTE in patients undergoing total hip arthroplasty.

In RECORD2, extended duration rivaroxaban was compared to short term enoxaparin in patients undergoing total hip arthroplasty (Kakkar et al., 2008). Patients were randomised to receive rivaroxaban for 31 to 39 days or enoxaparin $40 \mathrm{mg}$ once a day for 10 to 14 days, and again underwent mandatory bilateral venography at the end of the treatment period. The primary efficacy outcome was the same as that in RECORD1. In the rivaroxaban group, $2.0 \%$ of the patients met the primary outcome variable compared to $9.3 \%$ in the LMWH group. 
Major venous thromboembolism occurred in $0.6 \%$ of the rivaroxaban group compared to $5.1 \%$ of the LMWH group. Major bleeding occurred in one patient in each group.

\section{Rivaroxaban: short term prophylaxis in total knee arthroplasty}

The third RECORD study assessed the efficacy and safety of 14 days treatment with rivaroxaban or enoxaparin for the prophylaxis of VTE in total knee arthroplasty (M. Lassen et al., 2008). 2,531 patients were randomised. In the 1702 patients analysed for the primary outcome variable, $9.6 \%$ of patients in the rivaroxaban group were diagnosed with DVT or PE, or died compared to $18.9 \%$ in the LMWH group. In the 1,833 analysed for the secondary outcome variable, $1 \%$ in the rivaroxaban group and $2.6 \%$ in the LMWH group met the criteria of major VTE (proximal DVT, nonfatal PE, or death related to VTE) and / or symptomatic VTE. Major bleeding, the main safety outcome variable was defined as bleeding that was fatal, that involved a critical organ, or that required reoperation or clinically overt bleeding outside the surgical site that was associated with a decrease in the haemoglobin level of $20 \mathrm{~g} / \mathrm{L}$ or requiring infusion of 2 or more units of blood. This occurred in $0.6 \%$ and $0.5 \%$ of patients in the rivaroxaban and LMWH groups respectively.

In all the RECORD studies rivaroxaban appears a more effective treatment then LMWH for the prevention of VTE in the clinical setting of total hip arthroplasty and total knee joint replacement, but it should be noted that the primary outcome variables and main secondary outcome variables were composites of a number of end points. The use of composite variables is beneficial in avoiding adjustment for type 1 error due to multiple tests, and can reduce sample sizes in studies by increasing the control rate of events of interest (Freemantle, Calvert, Wood, Eastaugh, \& Griffin, 2003). The inherent problem with composite variables is that it may be hard to tease out which component has the greatest, or perhaps the only significant effect. In each of the RECORD studies the significant findings were appeared to be driven by the comparative rates of DVT between the two groups. In none of the studies was the difference in the rate of non-fatal PE or all-cause mortality statistically different. 


\section{Meta-analysis of rivaroxaban RECORD trials}

In a meta-analysis of data from RECORD1, 2 and 3 a different composite primary outcome variable was applied; symptomatic VTE (both DVT and non-fatal PE) and all-cause mortality in the LMWH controlled period (B. I. Eriksson et al., 2009). The secondary outcome variable was the same combination of events until the end of the planned treatment periods ( 5 weeks in RECORD 1 and 2, and 2 weeks in RECORD 3). For assessment of safety, another combined outcome variable was used, major bleeding; fatal, into a critical site such as retroperitoneal, intracranial, intraocular, or intraspinal, requiring re-operation, or clinically overt extra-surgical site bleeding associated with a fall in haemoglobin of $\geq 20 \mathrm{~g} / \mathrm{L}$ or requiring infusion of 2 or more units of whole blood or packed cells. $0.4 \%$ of the rivaroxaban group and $0.8 \%$ of the LMWH group suffered symptomatic VTE or all-cause mortality at two weeks, and $0.5 \%$ and $1.3 \%$ respectively, suffered VTE or all-cause mortality at the end of the treatment period. Major bleeding events were not significantly different between the two groups.

As a result of these studies, rivaroxaban is now seen as an effective and safe alternative to LMWH for the prevention of VTE in the orthopaedic setting. With a reported compliance rate of $94 \%$ in hospital and at least $83 \%$ compliance at home over a six week period (Carrothers et al., 2014), rivaroxaban may lend itself to a role in the prophylaxis of VTE in the outpatient setting of lower limb immobilisation.

\section{Rivaroxaban in lower limb immobilisation}

While there have been no RCTs completed of rivaroxaban versus standard of care for patients undergoing lower limb immobilisation, there are reports in the medical literature of the use of rivaroxaban in those patients with lower limb immobilisation who are deemed 'high risk' for VTE. Haque and Davies have reported on a retrospective review of the incidence of VTE within their own hospital for a series of 200 patients that presented conservatively treated ankle fractures managed in the outpatient setting (Haque \& Davies, 2015). In accordance with hospital guidelines, 52 patients with at least one other risk factor for VTE (high BMI, OCP use, recent travel history, active cancer, family history of VTE and clotting disorders) were categorised as high risk of VTE and started on rivaroxaban. Those 
who had no other risks except lower limb immobilisation were offered no prophylaxis. There was one episode of asymptomatic IDDVT in the non-rivaroxaban group, and no symptomatic VTEs in the rivaroxaban group. Unfortunately there is no discussion of or comparison to the prophylactic regime (if any) or the VTE incidence in this patient group prior to the implementation of rivaroxaban for the high risk patients. From the data presented, the authors concluded that rivaroxaban was a safe and effective medication for the prevention of VTE in the setting of outpatient lower limb immobilisation. 


\section{Aspirin}

Aspirin (acetasalicylic acid) is a non-steroidal anti-inflammatory medication with antiplatelet effects. Aspirin causes inhibition cyclo-oxygenase, an enzyme in the production pathway of platelet thromboxane A2 (TxA2). TxA2 is essential for activating platelet aggregation and this is considered to have a role in the prevention and treatment of VTE. Prior to 1994 there had been a range of trials assessing VTE outcomes in medical and surgical patients, with varying results.

\section{Antiplatelet Trialists' Collaboration}

In 1994, the Antiplatelet Trialists' Collaboration reported on a meta-analysis of 80 trials, 53 of which involved patients receiving aspirin or control in general or orthopaedic surgery, nine trials of patients receiving aspirin or control in other types of immobility and 18 trials of aspirin versus another antiplatelet regimen (Antiplatelet Trialists' Collaboration, 1994). The duration of treatment in the trials ranged from two weeks to eight weeks.

On aggregate, $25 \%$ of those receiving aspirin were diagnosed with DVT on phlebography or systematic fibrinogen scanning, compared to $34 \%$ of controls resulting in a risk reduction of about $25 \%$ and an odds reduction of between $31 \%$ and $39 \%$. These proportional differences were the same in general surgery and orthopaedic surgery both traumatic and elective, with absolute reduction being greatest in those patients deemed at higher risk of developing VTE. In aspirin patients $1 \%$ were diagnosed with PE compared to $2.7 \%$ of controls which was also statistically significant.

Deaths attributed to PE were $0.2 \%$ in the aspirin groups and $0.9 \%$ in the control groups. However, in the aspirin group there was an excess of death due to 'other' causes, which meant that there was no significant different in overall mortality rates between the two groups. Significant bleeds requiring transfusion were more common in the aspirin groups than the control groups, as were complications (haematomas, infections and reoperations).

The authors found that on balance, aspirin halved the risk of DVT and PE in medical and surgical patients, and that the benefits accrued to those at higher risk of developing VTE. 
They recommended that aspirin be considered either alone or in conjunction with other treatment for the prevention of VTE in high risk groups.

\section{The Pulmonary Embolism Prevention Trial}

The Pulmonary Embolism Prevention trial followed, and was designed to 'confirm or refute' the findings of the Antiplatelet Trialists' Collaboration (Pulmonary Embolism Prevention (PEP) Trial Collaborative Group, 2000). While the effectiveness of inpatient LMWH treatment in reducing VTE events was noted, the authors commented that this treatment was often stopped at discharge even though the risk of VTE persisted for some weeks postoperatively. It was thought that aspirin could be cheaply and simply prescribed at discharge to allow for a longer period of prophylaxis. This study randomised 13,356 patients undergoing surgery for hip fracture and 4,088 patients undergoing hip arthroplasty to receive either $160 \mathrm{mg}$ aspirin daily or placebo, started pre-operatively and continued for 35 days post-operation. Patients also received whatever other thromboprophylaxis was considered necessary. VTE was diagnosed in $1.6 \%$ and $2.5 \%$ of the fracture patients assigned to aspirin and placebo respectively, a proportional reduction in VTE events of $36 \%$. There were 18 fatal PEs in the aspirin group compared to 43 in the placebo group. There were no differences in the rates of death due to bleeding, but there was a higher rate of bleeding episodes requiring transfusion in the aspirin group. The authors reported similar proportional differences in the hip arthroplasty group and concluded that aspirin should be considered for routine prolonged prophylaxis of VTE in surgical groups for the duration of the increased risk of VTE.

This study met with a substantial amount of criticism, with claims of a change in the primary outcome endpoints which were stated as DVT and PE in the final paper compared to 'all vascular deaths during days 0 to 35' and 'Major non-fatal vascular events (PE, myocardial infarction and stroke) in hospital and up to Day 35' in the published protocol (MacMahon, Rodgers, Collins, \& Farrell, 1994), and an over-inflation of the effects in arthroplasty rather than trauma related surgery.

That the reported end point in the paper included DVT which had not initially been included in the definition of major non-fatal vascular events was also contentious, as again, there was 
ongoing debate on the clinical significance of DVT. Importantly, these were DVT that were initially symptomatic and consequently radiologically diagnosed, so on balance, the reduction seen with aspirin appears to be true for the combination of DVT and PE in traumatic orthopaedic surgery after hospital discharge in a period where initial aggressive prophylaxis with other agents was usually stopped.

\section{Aspirin in lower limb immobilisation}

If aspirin is effective in reducing VTE events in high risk orthopaedic patients, it is also possible that it may reduce the risk of VTE in the clinical setting of lower limb immobilisation. Oral anticoagulation may well be preferred by patients compared to subcutaneous injections of LMWH, and is likely less costly to healthcare providers. While the PEP group recommended the use of aspirin for VTE prophylaxis in high risk medical groups, there was no mention made of ambulatory trauma and / or lower limb immobilisation. Trials reporting the use of aspirin for VTE prophylaxis in lower limb immobilisation are sparse. In 1998, Gehling reported a RCT of 500mg of aspirin twice a day versus a LMWH for the prevention of VTE in patients immobilised in bandages or casts (Gehling et al., 1998). Duplex sonography was undertaken on those who reported symptoms and / or at removal of the immobilisation. $4.8 \%$ of patients in the aspirin group and $6.3 \%$ in the LMWH group were found to have DVT. The rates reported in both arms of the Gehling trial are generally less than those reported in other prospective studies utilising radiological screening as outlined in Table 3.2 of Chapter Three, but unfortunately, without a control group it is difficult to make comment on the effectiveness of either intervention in reducing the risk of VTE compared to no intervention.

The Healy study of 2010 (B. Healy, Beasley, et al., 2010) conducted at Wellington Hospital, Capital and Coast District Health Board (CCDHB), between January 2006 and December $2007,13 / 208$ (6.3\%) of patients with Achilles tendon injury requiring lower limb immobilisation for a minimum of one week developed symptomatic VTE, presenting as DVT or PE (B. Healy, Beasley, et al., 2010). With the exception of one patient, no VTE prophylaxis was prescribed during the period of immobilisation 
Use of LMWH for this clinical situation is not funded in New Zealand, so a policy was set in place by the Orthopaedic Department that all patients who were managed with lower limb cast immobilisation, including but not limited to Achilles tendon injury, would receive a prescription for a six-week course of enteric coated aspirin, 100mg daily, for VTE prophylaxis, unless patient risk factors suggested that more aggressive prophylaxis was indicated.

This change provided me with an opportunity to undertake another review of VTE rates in Achilles tendon injury within the same environment to reconfirm the incidence in this patient group, and to assess whether the introduction of routine prophylactic aspirin for patients undergoing lower limb immobilisation has resulted in a change in VTE incidence. 


\section{STUDY FIVE: Venous thromboembolism rates in lower limb immobilisation after Achilles tendon injury unchanged after introduction of prophylactic aspirin: audit}

\section{Hypothesis}

The hypothesis of this study was that symptomatic VTE rates in patients with Achilles tendon injuries who are required to undergo lower limb immobilisation has decreased since the introduction of routine prophylactic aspirin for all patients undergoing lower limb immobilisation.

\section{Primary outcome variable}

The primary outcome variable is the proportion of patients presenting with Achilles injuries over a two year period who developed and symptomatic, and then radiologically confirmed VTE.

\section{Secondary outcome variables}

Secondary outcome variables included an assessment of the frequency of prescription of aspirin and other pharmacological prophylactic measures and a review of the clinical features of the VTE episodes.

\section{Methods}

I reviewed the hospital records of 218 patients aged 18 to 65 who attended the Orthopaedic Assessment Unit at Wellington Hospital between January 2013 and December 2014 with Achilles tendon injury requiring lower limb immobilisation for greater than one week. The incidence of VTE in the 70-day period following injury and its $95 \%$ confidence intervals were calculated using the Clopper-Pearson test. Simple descriptive statistics were used to summarise the patient characteristics.

\section{Note}

This study has been reproduced within the publication permissions (Appendix XII) of John Wiley and Sons publication group (Irene Braithwaite, Dunbar, Eathorne, Weatherall, \& 
Beasley, 2016). The introduction has been removed and the discussion expanded to accommodate an updated literature review. 


\section{Patients and Methods}

Hospital records were searched electronically to identify all those patients who were coded as a presentation to the acute Orthopaedic Assessment Unit or to the Emergency Department at Wellington Hospital, CCDHB between January 2013 and December 2014 with a confirmed injury to the Achilles tendon. This included partial and complete rupture, laceration, tendonitis, and tendinopathy. The International Classification for Disease (ICD-9) codes used were M766 and S860.

Patients were excluded from the audit if the Achilles tendon injury did not require immobilisation with a cast or moonboot, if immobilisation was less than one week, if they were outside of the age range (18 to 65 years), if the index event occurred before January 2013, if follow-up treatment occurred outside the CCDHB, or if the VTE event was not within 70 days of immobilisation. Patients who had multiple presentations with Achilles tendon injuries within the period in question had only the first event included in the audit.

Electronic and associated paper records were reviewed for information such as patient's demographic details, the nature of their Achilles tendon injury, whether management was conservative or operative, and what, if any, prophylaxis was prescribed. Where it was documented in the patient records that aspirin should be prescribed, but there was no copy of the aspirin prescription, it was taken that aspirin was in fact, prescribed. Risk factors for VTE identified in the patient records were also noted.

Hospital electronic health clinical records were then also searched to identify patients who were identified as having a VTE event after lower limb immobilisation associated with the index event. These events were found by reviewing clinic letters and radiology reports specific to each patient within 70 days of lower limb immobilisation. In order to be included in this subset, patients had to attend within 70 days of immobilisation, with a symptomatic VTE, with the diagnosis confirmed via Doppler ultrasound of the lower limb or CTPA.

Simple descriptive statistics are used to summarise the patient characteristics. The incidence of VTE in the 70 -day period following injury and its $95 \%$ confidence intervals were calculated 
using the Clopper-Pearson test. The audit was approved by the Quality and Risk Management Unit of the CCDHB. 


\section{Results}

There were 218 patients who met the inclusion criteria (Table 5.1). Achilles tendon rupture was the most common injury with 211 having a partial or complete rupture. Of the remaining, 5 patients suffered lacerations, and 2 suffered from tendonitis. All 218 patients were immobilised by cast or moonboot for a minimum of 7 days.

Table 5.1. Characteristics of patients with Achilles tendon injuries included in audit.

\begin{tabular}{lll} 
& \multicolumn{2}{c}{ VTE N (\%) } \\
& No (N=204) & Yes (N=14) \\
\hline Sex & & \\
Female & $80(39.2)$ & $7(50.0)$ \\
Male & $124(60.8)$ & $7(50.0)$ \\
Achilles tendon Injury & & \\
Laceration & $4(2.0)$ & $1(7.1)$ \\
Rupture & $198(97.1)$ & $13(92.9)$ \\
Tendonitis & $2(1.0)$ & $0(0)$ \\
\hline Operative management & & \\
\hline Non-operative & $168(82.4)$ & $12(85.7)$ \\
Within 7 days of index event & $12(5.9)$ & $1(7.1)$ \\
$>7$ days from index event & $24(11.8)$ & $1(7.1)$ \\
\hline Risk Factors for VTE documented at acute clinic & & \\
\hline No & $198(97.1)$ & $13(92.9)$ \\
Yes & $6(2.9)$ & $1(7.1)$ \\
\hline Aspirin Prescription & $199(97.5)$ & $14(100)$ \\
\hline Prescribed & $5(2.5)$ & $0(0)$ \\
\hline Not prescribed & $189(92.6)$ & $14(100)$ \\
\hline Other prophylaxis prescribed & $17(18.3)$ & $0(0)$ \\
\hline No & $15(7.4)$ & $0(0)$ \\
Yes & & \\
\hline VTE - & & \\
\hline
\end{tabular}

VTE - Venous thromboembolism 
All patients were initially conservatively treated with lower limb immobilisation on presentation. 13 patients had their treatment plan changed to operative within seven days of injury, and a further 25 were treated operatively more than seven days post injury due to failure of non-operative approach or re-rupture. Specific VTE risk factors identified at presentation to the orthopaedic clinic were documented in 7 patients (4 were to travel longdistance, 2 were pregnant, 1 had previously had a DVT).

There were 203 patients prescribed aspirin for VTE prophylaxis, including two who were already prescribed aspirin for other health reasons. For 17 of these patients, notes were made to prescribe aspirin, although a copy of the prescription could not be found. It was assumed for the purposes of analysis that these individuals did in fact receive aspirin. There were five patients who received prescriptions for alternative chemoprophylaxis (enoxaparin $[\mathrm{LMWH}] \mathrm{n}=4$; rivaroxaban [Factor Xa inhibitor] $\mathrm{n}=1$ ). There were 12 patients in whom there was no record of the prescription of any prophylaxis.

The mean duration of immobilisation could not be calculated from case notes however, routine CCDHB orthopaedic clinic policy following Achilles tendon rupture is six to eight weeks of immobilisation, regardless of whether the treatment method is surgical or nonsurgical (Table 5.2).

Table 5.2. Standard Capital and Coast District Health Board protocol for non-operative management of Achilles tendon injury by cast immobilisation

\begin{tabular}{lll} 
Time from injury or repair (wks) & Cast application & Mobility status \\
\hline 0 to 2 & Equinus cast & Non-weight-bearing \\
2 to 4 & Equinus cast & Non-weight-bearing \\
4 to 6 & Moonboot +3 heel raisers & Non-weight-bearing \\
6 to 8 & Moonboot +1 heel raiser & Initiating of weight-bearing exercises \\
$8+$ & Normal shoe +1 heel raiser & Normal weight-bearing \\
\hline
\end{tabular}


There were $14 / 218$ (6.4\%) patients, $95 \% \mathrm{Cl} 3.6 \%$ to $10.5 \%$, who presented with radiologically confirmed VTE events within 70 days of lower limb immobilisation (Table 5.3). The mean (SD) age of patients with a confirmed VTE event was 41 (8) years and in those without was 40 (12) years. Patients presented between one and seven weeks from initiation of cast immobility, and in only $1 / 14$ patients with a VTE was there a recognised risk factor documented. Surgery was undertaken in 2/14 patients who developed VTE, one within seven days of their injury, and one greater than seven days after their injury. Aspirin was prescribed to all patients who consequently developed a VTE, and none of these received any additional chemoprophylaxis. 12 patients were treated with bridging enoxaparin and warfarin, 1 was treated with enoxaparin and one was treated with the thrombin inhibitor dabigatran. 
Table 5.3. Characteristics of patients who experienced venous thromboembolism (VTE) within $\mathbf{7 0}$ days of cast immobilisation for treatment of Achilles tendon injury.

\begin{tabular}{llllllll}
$\begin{array}{l}\text { Age } \\
\text { (yrs) }\end{array}$ & Sex & $\begin{array}{l}\text { Aspirin } \\
\text { prescribed }\end{array}$ & $\begin{array}{l}\text { Alternative } \\
\text { prophylaxis }\end{array}$ & $\begin{array}{l}\text { Weeks } \\
\text { from index } \\
\text { event to } \\
\text { VTE event }\end{array}$ & Surgery & VTE event & $\begin{array}{l}\text { Risk factors } \\
\text { documented }\end{array}$ \\
\hline 31 & M & Yes & No & 2 & No & Distal DVT & Nil \\
31 & M & Yes & No & 6 & Yes & PE & Nil \\
32 & F & Yes & No & 3 & No & Distal DVT & Nil \\
34 & M & Yes & No & 1 & No & Distal DVT & Nil \\
35 & M & Yes & No & 2 & No & Distal DVT & Nil \\
39 & F & Yes & No & 3 & No & Proximal & Nil \\
& & & & & DVT & \\
40 & F & Yes & No & 4 & No & Distal DVT & Nil \\
41 & F & Yes & No & 4 & No & Distal DVT & Nil \\
41 & F & Yes & No & 3 & No & Proximal & Previous VTE \\
& & & & & & DVT & \\
42 & M & Yes & No & 1 & No & Distal DVT & Nil \\
44 & F & Yes & No & 4 & Yes & Distal DVT & Nil \\
48 & F & Yes & No & 1 & No & Distal DVT & Nil \\
50 & M & Yes & No & 1 & No & Distal DVT & Nil \\
54 & M & Yes & No & 7 & No & Distal DVT / & Nil \\
& & & & & PE & \\
VTE Ves & & & & &
\end{tabular}

VTE - Venous thromboembolism

DVT - Deep vein thrombosis

PE - pulmonary embolism 


\section{Discussion}

In this audit, $6.4 \%$ of patients aged between 18 and 65 years with an Achilles tendon injury managed by cast immobilisation suffered a symptomatic DVT or PE that was confirmed radiologically. All of these patients were prescribed $100 \mathrm{mg}$ of enteric coated aspirin daily for chemoprophylaxis of VTE. In all, $93 \%$ of patients received aspirin and $3 \%$ an alternative chemoprophylactic agent, reflecting a major change in practice since our last audit when only one patient of 208 (0.5\%) was prescribed aspirin (B. Healy, Beasley, et al., 2010). The $6.4 \%$ incidence of symptomatic VTE was similar to that observed in our previous audit, utilising the same criteria and methodology, in which $6.3 \%$ of patients who underwent lower limb immobilisation for Achilles tendon injury and who received no prophylaxis developed symptomatic VTE. This suggests that despite aspirin having some efficacy in reducing the risk of VTE following high risk orthopaedic surgery (Pulmonary Embolism Prevention (PEP) Trial Collaborative Group - 2000), it is insufficient to substantially reduce the VTE risk associated with lower limb immobilisation for Achilles tendon injury.

The Pulmonary Embolism Prevention (PEP) Trial (Pulmonary Embolism Prevention (PEP) Trial Collaborative Group - 2000) perhaps provides the strongest evidence for the efficacy of aspirin in the prevention of DVT in an orthopaedic setting. A total of 17,444 patients who had undergone surgery for hip fracture and arthroplasty were prescribed either 160mg of aspirin daily or placebo started pre-operatively and continued for 35 days post-operatively. The study failed to show any benefit of aspirin in the prevention of non-fatal PEs, but did show a modest $28 \%$ reduction in the risk of developing a symptomatic DVT after hip surgery. However, there has since been debate over the primary outcome end points, the final sample size calculation used to report the results, and the reporting of the bleeding endpoints in the PEP study (A Cohen \& Quinlan, 2000). The American College of Chest Physicians (ACCP) Evidence-Based Clinical Practice Guidelines for prevention of VTE in orthopaedic surgery patients (Falck-Ytter et al., 2012) conclude that while the PEP study showed a modest reduction in the relative risk of symptomatic DVT after high risk hip surgery, there was a close balance between 'desirable and undesirable effects'. 
While the results of this study do not preclude a possible reduction in risk of VTE since the prescription of aspirin, the rate of symptomatic VTE indicates that a substantial potentially preventable burden of disease still occurs despite its use. The high risk of VTE post Achilles tendon injury and subsequent lower limb immobilisation is likely to be due to numerous factors including the combination of soft tissue trauma and swelling, immobilisation of the plantar venous plexus and the calf muscles, as well as the general immobilisation of the patient. Thus a greater degree of prophylaxis may be required than aspirin alone.

In this study, the rates of VTE found after Achilles injury are within the range of symptomatic, radiologically confirmed VTE noted following lower limb immobilisation reported in the medical literature. Meek and colleagues reported an incidence of symptomatic VTE between 3.1 and $7.1 \%$ in patients aged $>18$ years who underwent rigid lower limb immobilisation for any cause (Meek \& Tong, 2012). Saragas and colleagues reported that $5.7 \%$ of patients undergoing surgical repair of their Achilles tendon, followed by cast immobilisation had a symptomatic VTE (Saragas \& Ferrao, 2011). The highest incidence of symptomatic VTE was reported by Makhdom and colleagues, in which $23.5 \%$ of patients who had ruptured their Achilles tendon and were treated surgically developed this complication (Makhdom, Cota, Saran, \& Chaytor, 2013). The lowest rate (0.8\%) was found by Patel and colleagues in a retrospective review of patients who had suffered Achilles tendon injury (A Patel et al., 2012).

To add further context, the most recent Cochrane meta-analysis of RCTs of LMWH for prevention of VTE in patients with lower-leg immobilisation reports a mean incidence of VTE of $18 \%$ with a range of between $4 \%$ and $40 \%$ in the placebo groups (Mark Testroote et al., 2014). The higher rates in this systematic review are due to the included studies utilising radiological screening for VTE events, rather than the retrospective review of medical records for symptomatic events. This compares with a mean rate of VTE as identified by radiological screening following hip and knee surgery without prophylaxis of $49 \%$, with a range of between $31 \%$ and $56 \%$ (L. N. Jorgensen et al., 1993).

The ongoing uncertainly around prevention of VTE in this clinical setting, despite the previously proven efficacy of LMWH is highlighted by the disparate guidelines addressing 
the prophylaxis of VTE in lower limb immobilisation. The ACCP regularly update guidelines for VTE prophylaxis in a number of medical and surgical settings. In the 2004 guidelines they cited an incidence of asymptomatic DVT in patients with below knee injuries of 10 to $40 \%$, but did not recommend prophylaxis as they were not convinced that LMWH prophylaxis would reduce the incidence of 'clinically important VTE' nor that use of LMWH was costeffective (Geerts et al., 2004). By 2012, the ACCP still issued no formal guidelines for the prophylaxis on VTE in lower limb immobilisation but note that further research was required in this clinical setting, particularly with respect to 'anticoagulant thromboprophylaxis versus aspirin stratified by type of injury and procedure and expected degree of immobility' (FalckYtter et al., 2012).

The National Institute of Health and Care Excellence (NICE) UK guidelines recommend considering prophylaxis for those in lower limb casts who already have at least one other risk factor for VTE (National Institute for Health and Clinical Excellence, 2010). In the Guidelines in the Emergency Medicine Network (GEMnet) it is noted that there is reasonable evidence to suggest a significant risk of VTE in patients undergoing lower limb immobilisation and that in the absence of a validated risk prediction score consideration should be given to routine prophylaxis with LMWH (Roberts et al., 2013). The Italian intersociety consensus statement on antithrombotic prophylaxis in orthopaedics and traumatology (Randelli et al., 2013) recommend LMWH for VTE prophylaxis in lower limb immobilisation or non-weight-bearing situations, until the patient is mobilised and ankle mobility and weight-bearing capacity are 'partially' restored.

In routine orthopaedic surgery, adjunctive mechanical prophylaxis, early mobilisation and active physiotherapy regimes are regular features of patient management in combination with chemoprophylaxis. None of these non-pharmacological approaches have been formally tested in outpatient management of patients with lower limb immobilisation. The inpatient period following orthopaedic surgery provides an opportunity to ensure prophylaxis is administered and that patients receive the supervision and advice they need to regularly mobilise and reduce the risk of VTE. These opportunities are lacking in the outpatient environment in which many patients with lower limb immobilisation are managed. 
A number of other potential medications (e.g. LMWH, Direct thrombin inhibitors (DTIs), Vitamin $\mathrm{K}$ antagonists, Factor Xa inhibitors), may prove efficacious in the prophylaxis of VTE in the setting of lower limb immobilisation. LMWH reduces the incidence of VTE following lower limb immobilisation (Mark Testroote et al., 2014) and thus represents a current evidence-based chemoprophylactic agent in this setting. However, LMWH is administered by subcutaneous injection, which may affect adherence to treatment in the outpatient setting, so alternative options should be explored. With vitamin $\mathrm{K}$ antagonists it can be difficult to achieve a therapeutic anticoagulation range and regular blood tests are required. The efficacy and safety of DTIs or Factor Xa inhibitors have not yet been assessed and clinical trials in the setting of lower limb immobilisation are a high priority.

Regardless of the disagreement over the risk and / or risk stratification for VTE in patients undergoing lower limb immobilisation or the clinical significance of asymptomatic DVTs compared to 'clinically significant' VTEs in this diverse patient group, LMWH is not funded by the government funding authorities in New Zealand for the primary prevention of VTE in this clinical setting and so current New Zealand practice is not to routinely use LMWH for VTE prophylaxis in patients with lower limb immobilisation.

There are some important methodological issues to take into account when considering the results of this audit. I included only those patients aged between 18 and 65 years of age. While this allowed direct comparison with our previous audit, older patients who have an inherently higher risk of VTE, and who potentially have additional risk factors such as cancer and reduced mobility were excluded. Thus the incidence of VTE in this audit is likely to be understated when considering all-comers who undergo lower limb immobilisation for Achilles tendon injury. It is possible that a number of patients may have moved out of the hospital catchment area soon after presenting with their index event, and a VTE may have been recorded in another locality. However this number is likely to be low and have little impact on the overall incidence of VTE that was found. All Achilles tendon injuries treated at the hospital are seen in the orthopaedic fracture clinic, and I am confident that all presentations were captured through a review of hospital electronic records and fracture clinic acute lists. Similarly, all VTE events in the hospital catchment area are referred to VTE 
clinics or seen within the oncology department at the hospital, and all of these events are captured on electronic records, thus I can be certain all VTE events in this patient group were captured. I was unable to collect comprehensive data on bleeding episodes during the period of aspirin treatment in this patient group, thus I cannot report serious adverse events in this sample as a result of exposure to aspirin.

In conclusion, despite the routine prescription of aspirin to patients with Achilles tendon injuries undergoing lower limb immobilisation in our sample, there is a high risk of symptomatic VTE, with rates similar to the pre-aspirin era. Randomised controlled trials of other chemoprophylactic agents such as DTIs and Factor Xa inhibitors or mechanical prophylaxis such as intermittent pneumatic compression devices within lower limb casts are indicated to elicit the risk- benefit profile of these possible interventions in this outpatient population. 


\section{Intermittent pneumatic compression therapy.}

IPC of the legs has been shown to be effective in reducing VTE events in hospitalised patients. IPC can be applied to the thigh, calf or foot, or any combination thereof, of a patient and has been used in both surgical and medical settings. IPC achieves an antithrombotic effect through increased venous velocity, thus avoiding venous stasis, and also through stimulation of endogenous fibrinolysis (A J Comerota et al., 1997). The effectiveness of IPC alone for prevention of VTE has been demonstrated in a number of RCTs in the setting of general surgery and orthopaedics.

In a study of 77 surgical patients randomised to bilateral long anti-embolism stockings (TEDs) or long leg IPC for a minimum of 72 hours post-operatively or until the patient was ambulatory, DVT was seen in one (2.6\%) of the 38 IPC patients and four (10.2\%) of 39 patients allocated to TEDs. PE was diagnosed in one patient in each group (Caprini et al., 1983).This was compared to a 'control group' (96 individuals who had declined the study). The rate of non-fatal DVT in the control group was $21 \%$, of which $25 \%$ were proximal, including one fatal PE.

Around the same time, a study of 150 patients undergoing abdominal surgery was reported in which 50 patients were allocated to NMES of the calf, 50 to low-dose subcutaneous heparin, and 50 to TEDs and IPC for a minimum of 72 hours or until the patient was ambulatory to a maximum of two weeks (Nicolaides et al., 1983). Rates of DVT were 18\%, $9 \%$ and $4 \%$ in each of the respective groups, indicating that IPC and Teds were as effective as low-dose subcutaneous heparin.

In a RCT of calf and thigh compression in patients undergoing total hip joint replacement, venography diagnosed DVT occurred in $49 \%$ of patients who received no prophylaxis compared to $24 \%$ of patients allocated to IPC (Hull et al., 1990) for 14 days post-operatively. Proximal DVT occurred in $27 \%$ of controls compared to $14 \%$ of the IPC group. Notably, $36 \%$ of the DVTs diagnosed were not in the operated leg.

A meta-analysis of the four most commonly used prophylactic agents (aspirin, warfarin, LMWH and IPC) for VTE prophylaxis in total knee arthroplasty was reported in 2000 (G. H. 
Westrich, Haas, Mosca, \& Peterson, 2000). All studies included in the meta-analysis used routine radiological screening for the detection of DVT events, but not necessarily for the PE events. The incidence of DVT was $53 \%, 45 \%, 29 \%$ and $17 \%$ in the aspirin, warfarin, LMWH and IPC groups respectively. Asymptomatic PE was found in $11.7 \%, 8.2 \%$ and $6.3 \%$ of the aspirin, warfarin and IPC groups respectively; there were no LMWH studies that undertook routine lung scans. Symptomatic PEs were diagnosed in $1.3 \%, 0.4 \%, 0.5 \%$ and $0 \%$ of the aspirin, warfarin, LMWH and IPC groups respectively. While the relative effectiveness of IPC was demonstrated in this meta-analysis, the rates of DVT were still high, and the authors suggested more studies were needed to assess the efficacy of combination therapies. Such studies were not long in coming.

\section{Intermittent pneumatic compression therapy in combination with other prophylactic} agents

In 2003, Macdonald and colleagues found no difference in rates of VTE in patients undergoing craniotomy who were treated with either subcutaneous heparin and IPC therapy or LMWH and IPC therapy (Macdonald et al., 2003).

IPC therapy in conjunction with LMWH resulted in a VTE rate of $1.7 \%$ in patients aged 40 years or more undergoing abdominal surgery, compared to $5.3 \%$ of patients who received IPC therapy only (Turpie et al., 2007). The proximal DVT rate was $0.2 \%$ in the IPC and LMWH group versus $1.7 \%$ in the IPC group. Major bleeds occurred in $1.6 \%$ of the LMWH / IPC group compared to $0.2 \%$ of the IPC only group.

Studies were also completed in the orthopaedic setting. In 275 patients undergoing total knee arthroplasty randomised to either inpatient IPC therapy and aspirin for four weeks or inpatient IPC therapy and LMWH for four weeks, DVT rates were $14.1 \%$ and $17.8 \%$ in the IPC / LMWH and IPC / aspirin groups respectively (G. Westrich et al., 2006). This difference was not of statistical significance. The IPCs were used for a mean of 18 hours over the five postoperative days.

In a study of 270 patients undergoing knee or hip arthroplasty, all of whom received LMWH, 141 were also randomised to receive inpatient bilateral calf compression for the duration of 
their hospital stay (Edwards et al., 2008). In the 153 knee arthroplasty patients, 76 (49\%) were randomised to receive concomitant IPC therapy. Of these, $6.6 \%$ were diagnosed with DVT compared to $19.5 \%$ in the LMWH only group and this was statistically significant. In the hip arthroplasty group, 65/124 (52\%) were allocated to concomitant IPC. In the hip IPC/LMWH group there was one DVT compared to 2 DVTs in the LMWH only group; this was not statistically significant. The IPC devices were used for a mean of 20 hours per day in the knee patients and 21 hours per day in the hip patients, all of whom had a mean length of stay of three days.

For patients undergoing total hip arthroplasty who received ten days of bilateral calf IPC with or without $81 \mathrm{mg}$ aspirin daily (according to surgeon preference), compared to LMWH therapy alone, the rates of distal and proximal DVT diagnosed by radiological screening 10 to 12 days post-operatively were $3 \%$ and $2 \%$ respectively in the IPC / aspirin group and $3 \%$ and $1 \%$ in the LMWH group (Colwell et al., 2010). Major bleeding events were $0 \%$ and $6 \%$ in the IPC / aspirin group and LMWH group respectively. PE rates were $1 \%$ in both groups. Patients used the IPC device for a mean of 11 days (range one to 15 days), a mean of 221 total hours (range 24 to 321 hours) and a mean of 20 hours per day. The reasons for discontinuation included noise, skin rash and unpleasant warmth on the legs.

In one meta-analysis, IPC was shown to be more effective than no IPC, or than the application of TEDs in preventing DVT in hospitalised patients; $17.3 \%$ versus $16.7 \%$ respectively (Ho \& Tan, 2013) and in the reduction of PE; $1.2 \%$ versus $2.8 \%$, but without any significant effect on mortality. When compared to single pharmacological prophylaxis IPC was as effective in reducing PE and was associated with a reduced risk of bleeding. The efficacy of IPC in reducing DVT was less clear due the heterogeneity of the studies included in the analysis. Pharmacological prophylaxis in conjunction with IPC reduced the risk of DVT by nearly half, but appeared to have no further effect on the risk of PE compared with the use of IPC alone. Thus one can draw the conclusion that IPC is better for VTE prevention than no prophylaxis, or the use of TEDs alone, that it is comparable to single pharmacological interventions and that pharmacological intervention and IPC combined are more effective in reducing VTE risk. 
IPC therapy is a particularly attractive prophylactic option in patients who are at increased risk of bleeding, as there is no systemic anticoagulative effect, nor are there adverse reactions so often associated with anticoagulation therapy. The Cochrane review of 2011 confirmed these findings in their review of eleven studies which included 7,431 patients (Kakkos et al., 2011). The Cochrane team found that IPC plus any anticoagulant decreased the incidence of PE from $2.7 \%$ to $1.1 \%$ and DVT from $4 \%$ to $1.6 \%$. They also found that compared to anticoagulation alone, IPC and anticoagulation reduced the incidence of DVT from $4.2 \%$ to $0.65 \%$. The rates of PE in this instance could not be determined due to lack of events in the studies included in the meta-analysis. 


\section{Intermittent pneumatic compression utilising foot pumps}

In 1983, Gardner and Fox described the 'venous foot pump', a physiological pumping mechanism of the sole of the foot which is activated by walking and has a stroke volume of 20 to $30 \mathrm{ml}$ (Gardner \& Fox, 1992). Comprising of large venae comites of the lateral plantar artery, the acting of walking causes compression of the veins forcing blood out at a pressure of $100 \mathrm{mmHg}$ up into the deep veins of the calf. Gardner and fox went on to describe the development of an Arterio-Venous ( $A-V)$ impulse device that wraps around the mid-foot, inflating rapidly under the solar venous plexus 'mimicking ambulation'. Noting that such a device might fit under plaster cast, Gardner and Fox suggested that even such a small device would be useful in reducing limb pain and swelling due to trauma and increasing venous flow in the immobilised patient.

In 1990, Wilson and colleagues described a prospective RCT of the A-V foot pump in total knee replacement surgery (Wilson et al., 1990). 59 patients were randomised to either bilateral foot compression or no intervention for 10 days post-operatively, and underwent ipsilateral venography to assess the incidence of DVT. The foot pump device reduced the incidence of 'major thrombosis' (major calf vein DVT and / or proximal DVT) from $60 \%$ in the control group to $18 \%$ in the intervention group. Proximal DVT rates were $19 \%$ in the control group and 0\% in the intervention group. Overall DVT rates were $69 \%$ in the control group and $50 \%$ in the IPC group. There was no discussion of patient adherence, bleeding events or adverse events related to IPC treatment.

In 1992, a prospective RCT of 84 patients undergoing total hip arthroplasty was reported (Fordyce \& Ling, 1992). All patients wore graduated compression stockings, practiced active leg exercises post-operatively and were mobilised on the second day. The 39 patients allocated to the A-V device were encouraged to use the device whenever they were in bed or sitting. Ascending venography was undertaken between day 6 and day 9 post-operatively. There were 16 patients in the control group and two patients in the A-V group who developed clinically significant thrombosis (proximal DVT, and major calf thrombosis; a thrombosis longer than $5 \mathrm{~cm}$ ). When minor DVT, (calf thrombosis less than $5 \mathrm{~cm}$ in length), 
was also included, 16 patients and four patients were diagnosed with DVT in the control and A-V groups respectively.

In 1993, Bradley and colleagues highlighted the benefits of A-V venous compression as an adjunctive therapy to their standard VTE prophylaxis regime of thigh-length anti-embolic stockings, twice daily heparin and twice daily hydroxychloroquine sulphate (Bradley, Krugener, \& Jager, 1993). After performing bilateral ascending venography 12 days after the operation, $6.6 \%$ of the patients in the $A-V$ group and $27.3 \%$ of the patients in the nonpumped group were diagnosed with DVT. There were no serious complications associated with the pharmacological prophylaxis. Two of the A-V patients developed pressure sores due to ill-fitting footwear, which was consequently modified without further problems.

One year later Santori and colleagues reported their RCT of 132 patients undergoing total hip arthroplasty. All patients wore compression stockings, 65 were treated with heparin and 67 were randomised to the A-V device. The incidence of DVT in the heparin group was $35.4 \%$, two thirds were major and one third were minor. One of the patients in the heparin group suffered a fatal PE. In the A-V group there were nine DVTs, three major and six minor, with no fatal PEs. There were no bleeding complications in the A-V group, compared to nine in the heparin group.

In summary, a foot pump appears an effective device for reducing the risk of VTE in the orthopaedic setting, without the additional risk of bleeding events that is encountered with chemical prophylaxis. Is it possible then, to place an IPC foot pump within a lower limb cast as a potential preventive measure for VTE?

\section{Mechanical prophylaxis of venous thromboembolism in lower limb immobilisation}

At the time of developing Study Six, there were few reports in the medical literature of mechanical prophylaxis for VTE within a lower limb cast. In 2006, a water filled bladder was tested within a lower limb plaster cast (Poelkens et al., 2006). The bladder ran down the dorsal side of the calf and to beneath the sole of the foot and could contain between 50 and $150 \mathrm{ml}$ of fluid. The idea was that the water would move with gravity, compressing the plantar venous plexus as patients became upright, and as the lower limb went through the 
'swing phase' associated with using crutches. This was effective in improving ejection flow in the lower limb during patient mobilisation, but will have no effect during periods of immobility. There have been no studies of the feasibility of this system in the context of prolonged lower limb immobilisation.

A NMES device has also been trialled within a below knee cast (Warwick et al., 2013). Prior to placement within a lower limb cast, the device was attached over the peroneal nerve, and the strength of the stimulation was modified until the participants' foot twitched. The device was then placed beneath a below-knee cast. Compared with no stimulation, blood flow measured in the femoral vein was improved by 18 to $131 \%$. Participants reported only mild discomfort while using the device. However, mild discomfort over a protracted period of time may hinder patient adherence to the treatment, and there was no discussion of NMES use or care when the cast might become wet.

There has been one study of an IPC within a below knee plaster cast in post-operative Achilles repair patients (Domeij-Arverud, Latifi, Labruto, Nilsson, \& Ackermann, 2013). In this study, the plastic foot garment was applied directly against the skin of the foot and beneath a plaster cast. Patients were asked to use the device for a minimum of six hours per day for a maximum of two weeks post-operatively. The study was stopped early due to high rates of DVT detected by ultrasonography in both the IPC and non-IPC groups. Only 14 patients were randomised to the IPC intervention, and two withdrew due to poor tolerance of the device. Seven of the remaining 12 patients encountered problems with incomplete foot compression within the cast and needed new casts placed within the two week treatment period.

The findings from the Domeij-Averud study suggest that the feasibility of IPC within lower limb casts is dependent on a number of factors. Firstly, such a device must be able to operate with a degree of reliability and certainty within the lower limb cast. The foot garment cannot be too bulky, expanding the cast over time as it inflates, which could either impact the integrity of the applied cast over time or the ability of the device to achieve and maintain pressure. Secondly, the patients must be able to tolerate the device. It is unclear in this study why or how the two participants found the device intolerable, but the application 
of a plastic garment directly to the skin beneath a plaster cast which can become warm and damp over time may have caused issues with skin maceration, itching or ulceration. Thirdly, it is important to understand whether the foot garment operates as it should within a rigid containment system. The foot garment used in the Domeij-Averud study has been designed to work within a soft garment, and it's possible that the haemodynamic performance of the IPC system was altered in some way by placement within a plaster cast, contributing to the number of patients complaining of device malfunction.

In early 2015 the same research team reported the results of a very similar study, where instead of a plaster cast, 74 patients with surgical repair of the Achilles tendon were randomised to intermittent foot compression beneath the softer orthotic device developed and supplied by the same manufacturer of the foot garment. In all, 74 participants were randomised to the usual treatment of immobilisation within a plaster cast in the equinus position (Figure 5.1) (Domeij-Arverud et al., 2015). IPC and the orthosis were applied for two weeks at which time all patients were allowed to start full weight-bearing. Bilateral US was undertaken at two weeks and six weeks. At two weeks, $23 \%$ of all IPC patients ( $21 \%$ of all IPC protocol compliant patients) and $37 \%$ of control patients were diagnosed with DVT (for intention to treat; $\mathrm{OR} 2.11,95 \% \mathrm{Cl} .97$ to $4.62, \mathrm{p}=0.061$ and for per protocol; OR 2.60; $95 \%$ $\mathrm{Cl} 1.15$ to $5.91 ; p=0.022$ ). At six weeks post-operatively DVT was diagnosed in $52 \%$ of the patients in the IPC group and in $48 \%$ of the patients in the control group (OR $0.94 ; 95 \% \mathrm{Cl}$ 0.49 to 1.83$)$.

My original intention was to assess whether the IPC within a lower-limb cast would be a feasible treatment arm in a three-arm RCT of $100 \mathrm{mg}$ aspirin daily versus IPC plus $100 \mathrm{mg}$ aspirin daily, versus rivaroxaban $10 \mathrm{mg}$ daily for the prevention of VTE in prolonged lower limb immobilisation. Seeing the original study successfully completed with a soft orthosis, I consulted with my orthopaedic colleague and found that the Vacoped Velcro cast was not considered suitable for long term immobilisation of orthopaedic patients as it may not provide an acceptable amount of rigid immobilisation for all clinical situations and it may be to too easy for patients to remove. 
I hypothesised that given the success of the Domeij-Averuid study using the soft orthosis, use of a more malleable fibreglass cast might allow more flexibility and cast rebound for the foot garment to inflate in compared to a plaster cast and that close attention to skin care might ameliorate patient discomfort. I also hypothesised that the IPC device might have to be used for the entire period of lower limb immobilisation, as at six weeks the DVT rates between the two treatment groups in the Domeij-Averud study using the soft orthosis were the same, suggesting that the efficacy of the IPC device did not extend beyond its immediate use. With a view to undertaking a similar study of the same IPC device under a fibreglass cast for up to six weeks duration, I undertook a venous haemodynamic study to compare the performance of the IPC system both within and without a fibreglass cast to ensure that device performance was not impeded, nor that it created excessive pressures through its placement in a lower limb cast.

The IPC system used in the Dameij-Averud study is the Vena Jet pump (VADOplex, Germany) and its' associated under-cast foot garment within a lower limb cast. Originally designed to be worn under a large Velcro 'cast' called the VacoPed (VADOplex, 2016) (Figure 5.1). It is now marketed as Jet Impulse Technology (JIT) in New Zealand, by Dickson and Dickson Healthcare and is referred to for the purposes of this these is IPC/JIT. 
Figure 5.1. Vena Jet pump (VADOplex, Germany) and its' associated under-cast foot garment
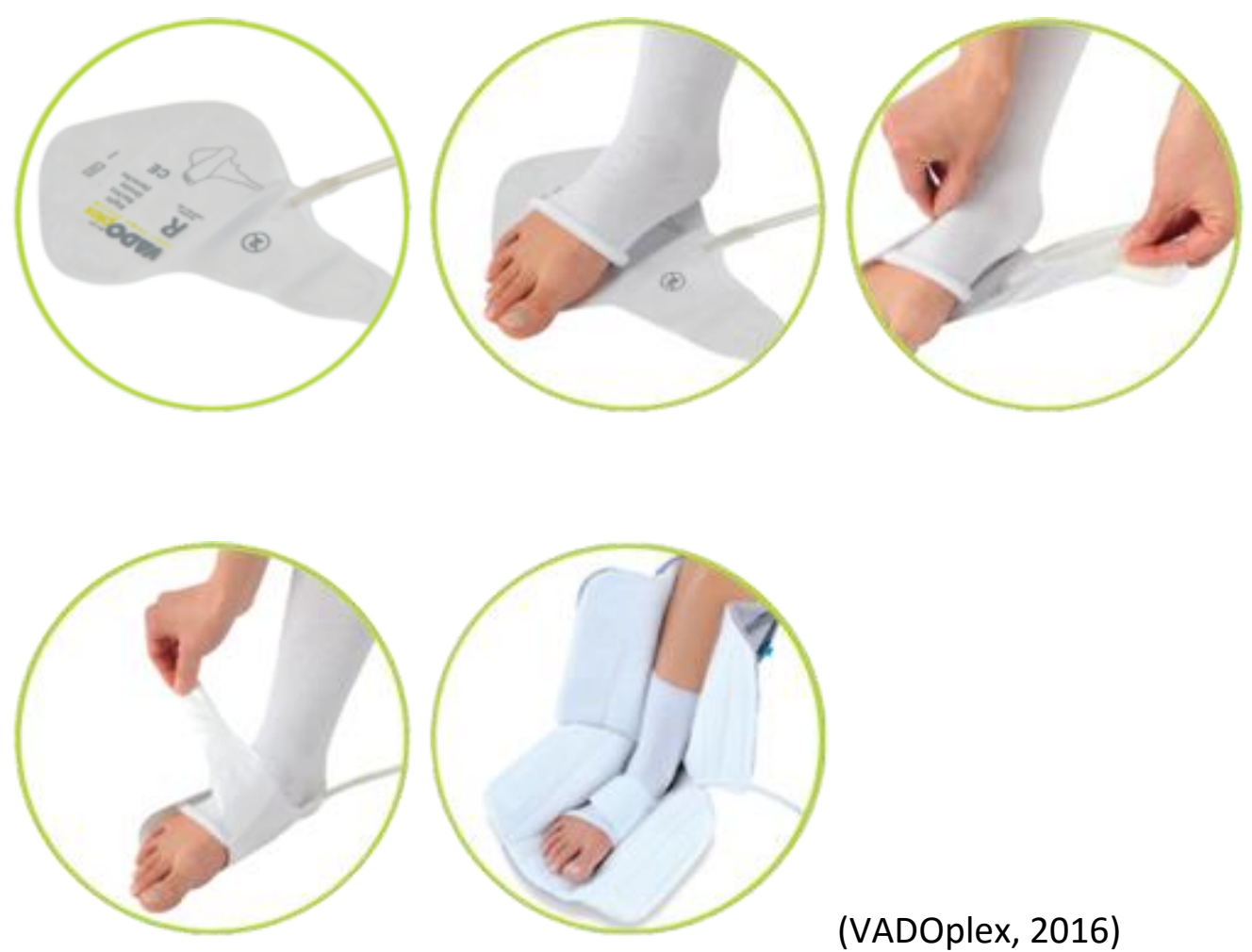

(VADOplex, 2016)

In conjunction with Sonographer Mr Steve Mackintosh and Fracture Clinic Nurse Miss Bernadette de Ruyter, we went through several permutations of possible cast placement that would allow the foot garment attachment to the pump machine to protrude through the cast and also allow measurement of the venous haemodynamics of the popliteal vein. The agreed cast for the haemodynamic study comprised several layers (Figures 5.2). The first layer was a tubular rib stocking from the dorsum of the foot to below the knee (Sutherland Medical, Victoria, Australia) on which self-adhering foam (Reston ${ }^{\mathrm{TM}}, 3 \mathrm{M}$, Minnesota, USA) was applied to the sole and the proximal dorsum of the foot and the medial malleolus. The foot garment was applied over the top of the adhesive padding with the attachment for the pump unit running up the padding on medial malleolus at which point it would protrude from the final cast. The third layer consisted of a soft pre-cast bandage (Soffban ${ }^{\circledR}$ Synthetic, BSN medical inc, North Carolina, USA) wrapping around the 
dorsum of the foot and up the lower leg to below the knee. The final layer consisted of fibreglass casting (Delta-Cast ${ }^{\circledR}$ Delta-Lite $^{\circledR}$, BSN medical inc, North Carolina, USA) (Figure 5.2). The top of the cast stopped approximately $50 \mathrm{~mm}$ distal to the popliteal crease behind the knee to allow access for the ultrasound probe. Once the casting process was tested and agreed, Miss de Ruyter then trained those clinic nurses that would be assisting us with the haemodynamic study. 
Figure 5.2: Application process, IPC and cast
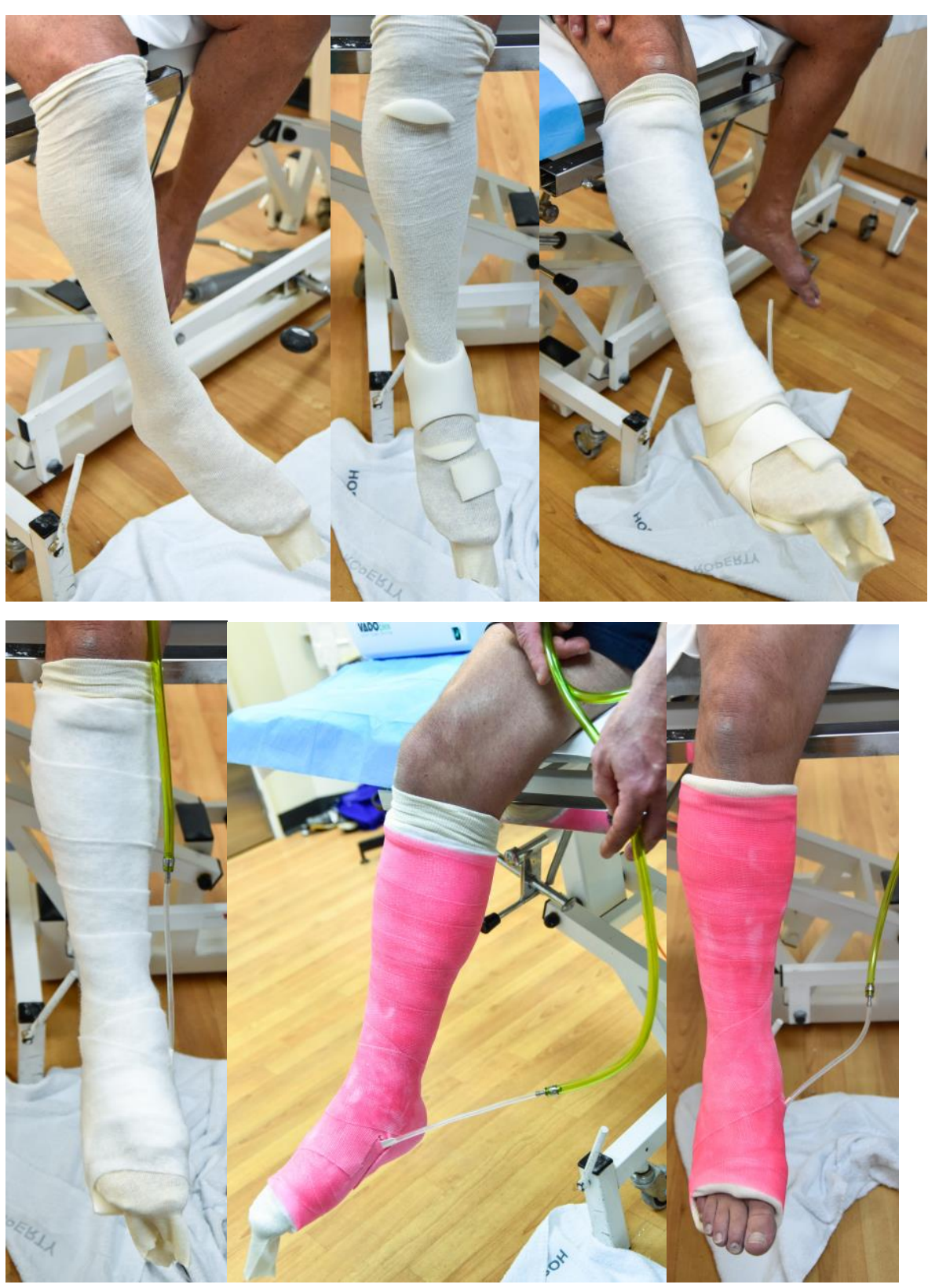


\section{STUDY SIX: Venous haemodynamics of Jet Impulse Technology within a lower limb fibreglass cast: a randomised controlled trial. (JIT in-cast trial)}

\section{Hypothesis}

The hypothesis for this study was that the VenaJet Jet Impulse Technology (IPC/JIT) system, usually designed to operate within a large Velcro bandage and / or unencumbered, was not impaired when placed within a below-knee fibreglass cast.

\section{Primary outcome variable}

The primary outcome variable was popliteal vein peak systolic velocity (PSV)(cm/s) compared between legs with and without the cast at 60 minutes (after ten minutes IPC/JIT activation).

\section{Secondary outcome variables}

Secondary outcome variables were the difference in PSV between the casted limb and the non-casted limb at baseline and 40 minutes after casting, and the difference in mean flow velocity $(\mathrm{cm} / \mathrm{s})$, vein diameter $(\mathrm{mm})$, and total volume flow $(\mathrm{L} / \mathrm{min})$ between the casted limb and the non-casted limb at baseline, 40 and 60 minutes.

\section{Methods}

This was a randomised controlled trial of 24 healthy participants aged 18 to 54 who had both feet placed within the IPC/JIT system and were randomised to have one or other leg (including the IPC/JIT) to be placed within a below-knee fibreglass cast. The analysis used a mixed linear model to estimate the overall difference between the IPC/JIT system within a cast and on the non-casted leg, as the control intervention. The baseline measurement was used as a continuous covariate, and the order of treatment, randomized leg, and sonographer were used as the other fixed effects, and the individual participants as random effects, to account for the paired design. 


\section{Methods}

In this randomised controlled trial, twenty four healthy volunteers aged between 18 and 65 years were recruited after attending a screening visit at the research office for review of inclusion and exclusion criteria and provision of informed consent. Potential participants were excluded if they had a history of previous or current deep vein thrombosis, were pregnant, or had clinical features of peripheral vascular disease, peripheral neuropathy, scleroderma, lymphoedema, or joint deformity from inflammatory arthritis. Participants who remained eligible were given appointments to attend a second visit for the study procedures.

At the second visit each participant was initially seated quietly for 10 minutes on the edge of an examination couch with both legs hanging dependent, as they would normally be seated to have their lower limb placed in an equinus lower limb cast. Baseline popliteal venous flow was measured at this time. Participants then had the IPC/JIT systems (Vena Jet, Germany) placed on both feet and were randomised to have either their left or right leg placed in a fibreglass below-knee equinus cast which also encased the IPC/JIT system on that limb.

Initially we intended that participants would remain in the initial position for the remainder of the study, but baseline popliteal venous flow measures were found to be very slow, and static in one participant, so participants were moved onto seats where they could rest at least part of their feet on the ground with the angle between the thigh and the lower leg at 120 degrees.

Participants were asked to sit forward enough on the chair that measurements with duplex ultrasonography could be performed without participants needing to move for the remainder of the study. Popliteal venous flow in this sitting position was re-measured $\mathbf{4 0}$ minutes after the randomised lower limb was placed in a cast. Then 50 minutes after the limb was placed in a cast the IPC/JIT system on both legs were activated to inflate to $130 \mathrm{mmHg}$ every minute. After 60 minutes, 10 minutes after IPC/JIT activation, popliteal venous flow was measured again and then the cast and IPC/JIT devices were removed. 
Imaging used a broadband linear ultrasound transducer (Philips IU22, Royal Philips, Eindhoven, The Netherlands) capable of both colour and pulsed Doppler. With a bandwidth of $3-9 \mathrm{MHz}$, pulsed Doppler imaging was acquired with a frequency of $3.5 \mathrm{MHz}$. The incident pulsed Doppler was beam steered to ensure a Doppler angle of less than 60 degrees to reduce errors in velocity calculation. The pulsed Doppler wall filter was reduced to capture low velocity flow. The sample volume was adjusted to encompass the entire diameter of the vein under investigation. Using triplex mode (live B-mode, colour and pulsed Doppler) the popliteal vein was sampled for flow. Auto-calculation measurements were made over a two second interval with phase being predominantly towards the heart. Cine-loop review allowed for vein diameter measurement (used to calculate volume flow) with good temporal resolution. Two sonographers were involved in the study, each measuring 12 participants in total. All measures of popliteal vein flow at each time point were undertaken twice in each limb, with the left limb measured first, and the mean of both measurements used in statistical analysis.

The primary outcome variable was the difference in PSV $(\mathrm{cm} / \mathrm{s})$, in the popliteal vein between the casted limb and the non-casted limb after 60 minutes, at which time the IPC/JIT system had been running for 10 minutes. Secondary outcome variables were the difference in PSV between the casted limb and the non-casted limb at baseline and 40 minutes after casting, and the difference in mean flow velocity $(\mathrm{cm} / \mathrm{s})$, vein diameter $(\mathrm{mm})$, and total volume flow ( $\mathrm{L} / \mathrm{min}$ ) between the casted limb and the non-casted limb at baseline, 40 minutes after casting and at 60 minutes.

The sample size was designed to rule out a paired difference in peak systolic flow of less than $4.8 \mathrm{~cm} / \mathrm{s}$. This non-inferiority bound is based on a past haemodynamic study where the mean maximum PSV achieved with an IPC outside of a fibreglass cast was $20.1 \mathrm{~cm} / \mathrm{s}$ with a paired SD for PSV of $6.8 \mathrm{~cm} / \mathrm{s}$ (Charles et al., 2013). The chosen non-inferiority bound of $4.8 \mathrm{~cm} / \mathrm{s}$ is $25 \%$ of the mean maximum PSV found in the past study. A sample size of 24 has $90 \%$ power with an alpha of $5 \%$ to rule out the nominated non-inferiority bound.

Participants were randomised to have a cast on one or other leg using a computer randomnumber based schedule developed by a biostatistician. The randomisation schedule was 
provided directly to a non-investigator, who prepared opaque envelopes for each study participant. The envelopes were opened by each participant immediately prior to the placement of the IPC/JIT systems and equinus cast.

The analysis used a mixed linear model to estimate the overall difference between the IPC/JIT system within a cast and on the other leg, as the control intervention. The baseline measurement was used as a continuous covariate, and the order of treatment, randomized leg, and sonographer as the other fixed effects, and the individual participants as random effects, to account for the paired design.

SAS version 9.3 was used for statistical analysis.

Ethics approval was obtained from the New Zealand Health and Disability Ethics Committee, ref 14/STH/82 20394, dated 03/07/2014 and the trial was prospectively registered with the Australia New Zealand Clinical Trials Registry: ANZCTR 12614000730606. 


\section{Results}

The flow of participants is shown in Figure 5.3. The age range for the participants was 18 to 54 years and 17 (71\%) were women. Mean (range) BMI was 25.9 (19 to 36 ) kg/m². One participant had no measurable blood flow in the leg randomised to be placed in a cast while in the baseline sitting position and was withdrawn from further participation in the study, although the baseline measurements in the leg without the cast were able to be used for analysis.

Figure 5.3. Flow of participants through study

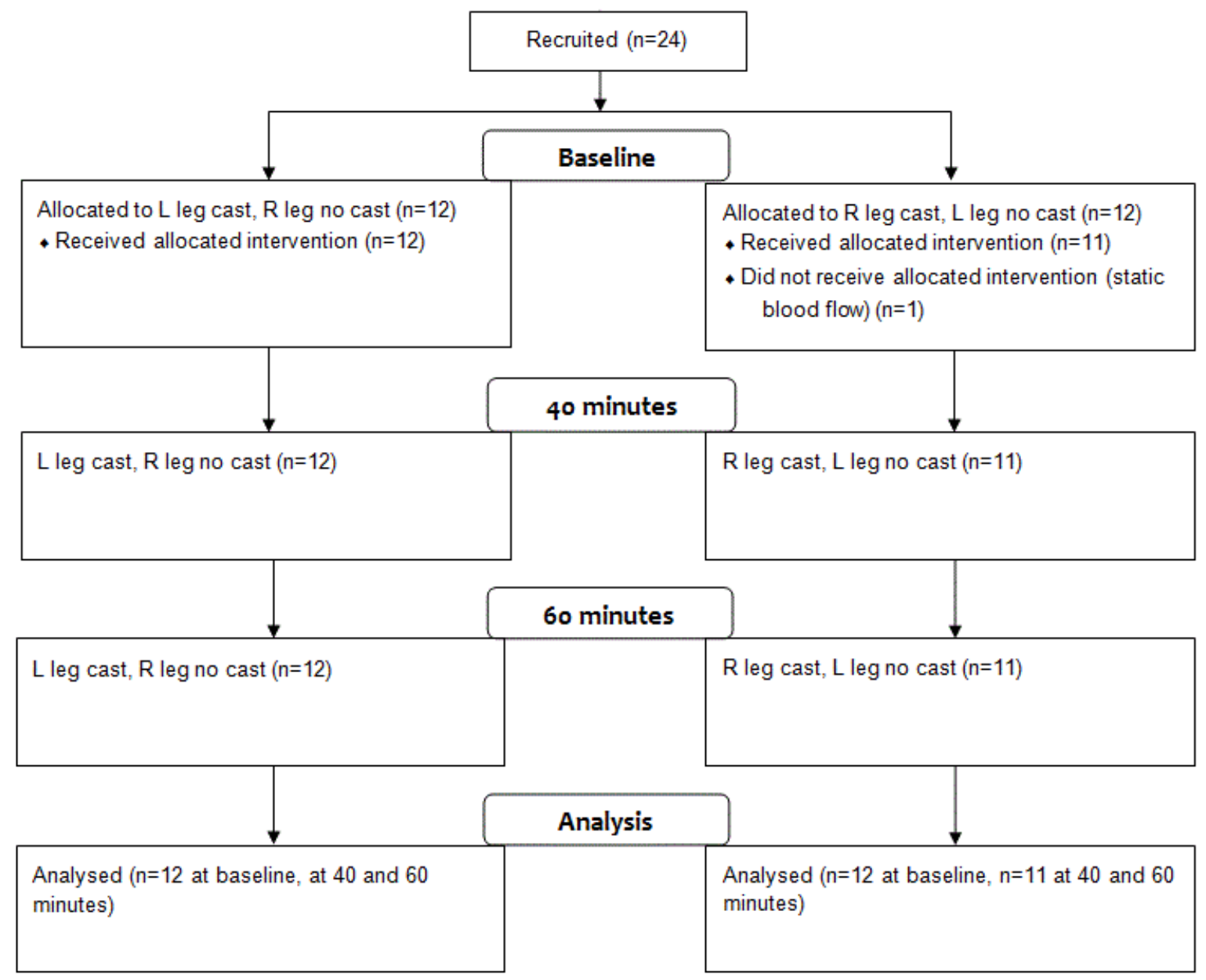


Table 5.4 shows summaries of popliteal vein blood flow. The mean (SD) PSV after 60 minutes, when the JIT system had been running for 10 minutes, was $28.8(16.1) \mathrm{cm} / \mathrm{s}$ and $29.3(19.0) \mathrm{cm} / \mathrm{s}$ in the legs with and without the cast respectively, representing a 6.3 and 6.8 -fold increase from baseline

(Figure 5.4). Mean Flow Velocity and Total Volume Flow were increased after 60 minutes compared to baseline while Vein Diameter remained

unchanged.

Table 5.4: Popliteal vein blood flow measurements assessed by duplex ultrasonography.

\begin{tabular}{|c|c|c|c|c|c|c|}
\hline & \multicolumn{6}{|c|}{$\begin{array}{l}\text { Mean (SD) } \\
\text { [Range] }\end{array}$} \\
\hline & \multicolumn{3}{|c|}{ Leg in cast } & \multicolumn{3}{|c|}{ Leg not in cast } \\
\hline & $\begin{array}{l}\text { Baseline } \\
(n=23)^{1}\end{array}$ & $\begin{array}{l}40 \text { minutes } \\
(n=23)^{1}\end{array}$ & $\begin{array}{l}60 \text { minutes } \\
(n=23)^{1}\end{array}$ & $\begin{array}{l}\text { Baseline } \\
(n=24)\end{array}$ & $\begin{array}{l}40 \text { minutes } \\
(\mathrm{n}=23)^{1}\end{array}$ & $\begin{array}{l}60 \text { minutes } \\
(\mathrm{n}=23)^{1}\end{array}$ \\
\hline PSV $(\mathrm{cm} / \mathrm{s})$ & $\begin{array}{l}4.6(1.5) \\
{[3.1 \text { to } 10.1]}\end{array}$ & $\begin{array}{l}4.8(1.1) \\
{[3.1 \text { to } 7.7]}\end{array}$ & $\begin{array}{l}28.8(16.1) \\
{[8.6 \text { to } 65.2]}\end{array}$ & $\begin{array}{l}4.3(1.2) \\
{[2.8 \text { to } 6.8]}\end{array}$ & $\begin{array}{l}4.8(1.4) \\
{[3.0 \text { to } 7.6]}\end{array}$ & $\begin{array}{l}29.3(19.0) \\
{[8.4 \text { to } 72.8]}\end{array}$ \\
\hline Mean flow velocity $(\mathrm{cm} / \mathrm{s})$ & $\begin{array}{l}1.4(0.7) \\
{[0.4 \text { to } 3.1]}\end{array}$ & $\begin{array}{l}1.8(0.7) \\
{[0.4 \text { to } 3.5]}\end{array}$ & $\begin{array}{l}4.8(3.3) \\
{[1.6 \text { to } 14.7]}\end{array}$ & $\begin{array}{l}1.4(0.7) \\
{[0.3 \text { to } 2.8]}\end{array}$ & $\begin{array}{l}1.7(0.9) \\
{[0.4 \text { to } 3.2]}\end{array}$ & $\begin{array}{l}3.8(2.4) \\
{[0.9 \text { to } 9.6]}\end{array}$ \\
\hline Total volume flow (L/min) & $\begin{array}{l}.048(.027) \\
{[.015 \text { to } .108]}\end{array}$ & $\begin{array}{l}.060(.025) \\
{[.014 \text { to } .106]}\end{array}$ & $\begin{array}{l}.172(.140) \\
{[.031 \text { to } .598]}\end{array}$ & $\begin{array}{l}.044(.023) \\
{[.009 \text { to } .104]}\end{array}$ & $\begin{array}{l}.053(.033) \\
{[.008 \text { to } .128]}\end{array}$ & $\begin{array}{l}.133(.095) \\
{[.027 \text { to } .411]}\end{array}$ \\
\hline Vein diameter $(\mathrm{cm})$ & $\begin{array}{l}0.85(0.13) \\
{[0.57 \text { to } 1.06]}\end{array}$ & $\begin{array}{l}0.85(0.12) \\
{[0.60 \text { to } 1.04]}\end{array}$ & $\begin{array}{l}0.85(0.13) \\
{[0.61 \text { to } 1.06]}\end{array}$ & $\begin{array}{l}0.83(0.11) \\
{[0.63 \text { to } 1.00]}\end{array}$ & $\begin{array}{l}0.82(0.15) \\
{[0.51 \text { to } 1.03]}\end{array}$ & $\begin{array}{l}0.85(0.14) \\
{[0.57 \text { to } 1.21]}\end{array}$ \\
\hline
\end{tabular}

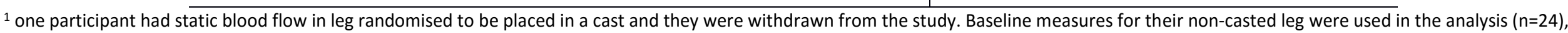
but no other measures were analysed ( $(n=23)$ for remaining time points).

SD - Standard deviation

PSV - Peak systolic velocity 
Figure 5.4: Box-plot of peak systolic velocity in casted and non-casted leg at each time point. The horizontal lines are the $25^{\text {th }}$, median and $75^{\text {th }}$ percentile, the symbol is the mean and the whiskers are the minimum to maximum.

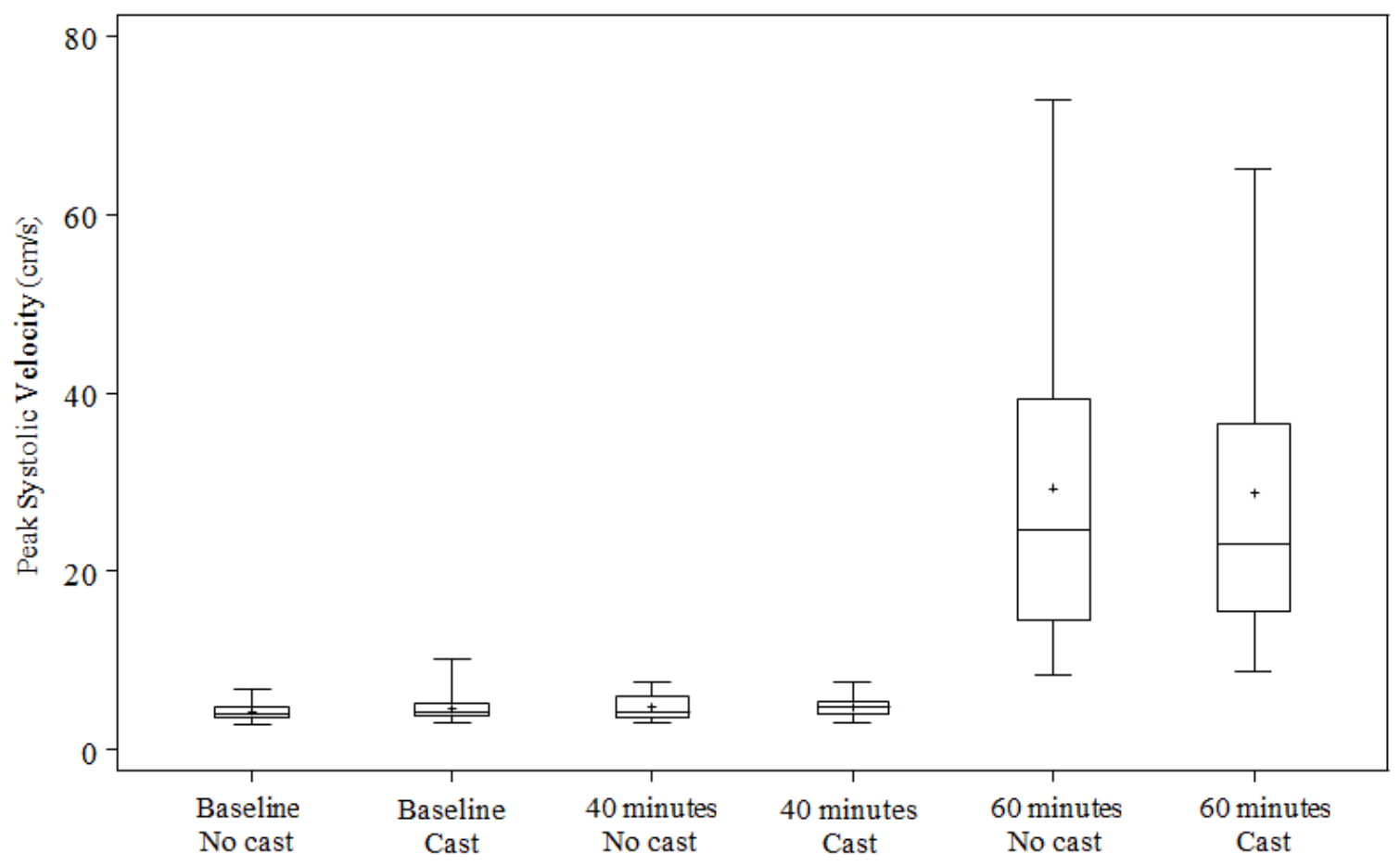


The mean (SD) and range PSV after 40 minutes was 4.8 (1.1), 3.1 to $7.7 \mathrm{~cm} / \mathrm{s}$; and 4.8 (1.4), 3.0 to $7.6 \mathrm{~cm} / \mathrm{s}$ in the legs with and without the cast respectively. These measurements as well as the mean flow velocity and total volume flow were not statistically significantly different from baseline. Differences in popliteal vein blood flow between the legs with and without the cast are shown in Table 5.5. There was no statistically significant difference between the legs with and without the cast for any measurement at any time point. 
Table 5.5: Differences in popliteal vein blood flow between casted leg and non-casted leg at each time point.

Mean (SD)

Cast minus no Change from baseline: Estimate $(95 \% \mathrm{Cl})$ of cast minus

cast

Cast minus no cast no cast,

P-value

\begin{tabular}{llll}
$\mathbf{4 0}$ minutes & & & \\
\hline PSV (cm/s) & $0.004(1.3)$ & $-0.2(2.1)$ & $-0.04(-0.66$ to 0.59$)$ \\
& & & 0.91 \\
& & $0.1(1.0)$ & $0.12(-0.26$ to 0.49$)$ \\
Mean flow & $0.1(0.8)$ & & 0.53 \\
velocity (cm/s) & & & $0.062(-0.076$ to 0.201$)$ \\
Total volume & $0.067(0.029)$ & $0.042(0.043)$ & 0.36 \\
flow (L/min) & & & $0.02(-0.02$ to 0.07$)$ \\
Vein diameter & $0.03(0.09)$ & $0.02(0.12)$ & 0.28 \\
(cm) & & &
\end{tabular}

60 minutes

\begin{tabular}{|c|c|c|c|}
\hline \multirow[t]{2}{*}{$\mathrm{PSV}(\mathrm{cm} / \mathrm{s})$} & $-0.4(12.7)$ & $-0.6(12.7)$ & $-0.8(-6.5$ to 4.9$)$ \\
\hline & & & 0.78 \\
\hline Mean flow & $1.0(2.6)$ & $1.0(2.6)$ & $0.96(-0.17$ to 2.1$)$ \\
\hline velocity $(\mathrm{cm} / \mathrm{s})$ & & & 0.09 \\
\hline Total volume & $0.386(0.121)$ & $0.362(0.117)$ & $0.365(-0.147$ to 0.876$)$ \\
\hline flow (L/min) & & & 0.15 \\
\hline Vein diameter & $-0.005(0.13)$ & $-0.02(0.14)$ & $-0.01(-0.07$ to 0.04$)$ \\
\hline$(\mathrm{cm})$ & & & 0.67 \\
\hline
\end{tabular}




\section{Discussion}

This study has shown that the effect of the IPC/JIT system on popliteal venous haemodynamics was not impaired by its use within a fibreglass below-knee cast. There was no statistically significant difference between the primary outcome of the PSV measured in the IPC/JIT system in legs with and without the cast after it was operating for 10 minutes, although the confidence interval for the IPC/JIT system PSV difference $(-6.5$ to $4.9 \mathrm{~cm} / \mathrm{s})$ just included the non-inferiority bound of 4.8. The lack of statistical power to exclude noninferiority of the system within the cast was because the SD of the measurements was double that anticipated based on our past research; $12.1 \mathrm{~cm} / \mathrm{s}$ compared to the anticipated SD of $6.8 \mathrm{~cm} / \mathrm{s}$. The PSV of $28.8 \mathrm{~cm} / \mathrm{s}$ measured in the leg with the cast and the IPC/JIT system represents a large (six-fold) change from baseline.

A number of methodological issues are relevant to the interpretation of the study findings. Venous haemodynamics were measured by Doppler ultrasound which is a non-invasive method of measuring lower limb blood flow that is sensitive, specific, and reproducible (de Groot, Bleeker, \& Hopman, 2005; Gaitini, 2006). PSV was chosen as the primary outcome variable because it is the most consistent non-artefactual wave form detected by ultrasound (de Groot et al., 2005). Although it is known that the right and left legs have similar venous haemodynamics with research participants (Wright \& Osborn, 1952), participants were randomised to have the JIT system placed on either the right or left leg to avoid any possibility of bias related to selection of the leg for casting, such as perceived discomfort which could be indirectly related to venous blood flow. Although it was not possible to blind the sonographers to the intervention allocation, this is unlikely to have resulted in biased assessment of blood flow.

The original research protocol was that all participants remained in the baseline position, with feet hanging dependent off the edge of the examination couch, for the duration of the study. This would have allowed the sonographer access to the popliteal vein, and placement of the equinus cast without having to move participants during the study. Unexpectedly there was very poor blood flow in this position, which resulted in withdrawal of one participant. Therefore the protocol was modified so that all participants were moved onto 
chairs and their feet could rest on the floor in order to relieve some of the compression of the femoral vein at the adductor hiatus. While every effort was made to ensure that the participants remained as still as possible after being moved from their baseline position, it cannot be guaranteed that this was achieved at all times. This may have resulted in the unexpectedly large SD seen in the study. This may actually improve the generalizability of the study however, as patients with injuries immobilised within a lower limb cast are unlikely to remain in a seated position without moving for prolonged periods of time.

I chose to investigate the IPC/JIT system in this trial as it is designed to be used within an already existing lower limb immobilisation system, and it delivers an inflation pressure $(130 \mathrm{mmHg})$ consistent with those delivered by foot IPC devices (Delis et al., 2000).While this pressure is at the lower end of the spectrum of previously studied foot IPC devices, this is likely to make the IPC/JIT system more tolerable when confined within a lower limb cast for between six and eight weeks. Past research also shows limited increases in PSV with increasing pressures delivered by foot IPC devices outside of a lower limb cast, so a higher pressure system may have produced little further clinical benefit (Delis et al., 2000). Use of a calf or calf and foot IPC within a cast is likely to be technically difficult and result in cast integrity issues over time.

The study was of 60 minutes duration, insufficient to measure any effect of the IPC/JIT system on the integrity of the cast over a protracted period of time. Now that I have shown the IPC/JIT system works at initial placement of a fibreglass cast, further studies are indicated to assess the longer term effects and tolerability of constant IPC/JIT activation within a lower limb fibreglass cast over periods of time reflective of those between orthopaedic outpatient visits. While the PSV is significantly increased by using the IPC/JIT system within a cast, consideration needs to be given to those issues that might limit its use in the clinical setting, including patient adherence to treatment, other aspects of tolerability, cost, patient skin integrity, and cast integrity with protracted periods of use in the outpatient setting.

Of possible concern, In the period between the design of this study and publication, Domeij and colleagues have published results of a trial of this same device within plaster lower limb 
casts (Domeij-Arverud et al., 2013). They found that in a group of 14 patients who underwent IPC treatment for a period of two weeks, two patients withdrew from the study as they did not tolerate the use of the device and nine of the remaining 12 were diagnosed with DVT when they underwent radiological screening. However the DVT diagnosis rate was not significantly different from the control group in which six of 12 patients were diagnosed with DVT. They noted that seven of the 12 participants felt that there were complications with the device during the two week period of treatment, and as a result, four patients received a new plaster cast. Placement of a second cast within that two week period correlated with increased risk of DVT, as did device malfunction. The study was terminated.

Late in 2015 , the same study group reported successful placement of the same device within the original orthosis for which it was originally designed. 150 post-surgical Achilles tendon repair patients were randomised to two weeks of treatment with the IPC device within the orthosis or placement as usual within a plaster cast (Domeij-Arverud et al., 2015). The study groups demonstrated a reduction in VTE rates with IPC treatment in the two weeks post-operatively. The ability to see this study through to completion within an orthosis rather than a plaster cast is reassuring. However, the orthosis can be easily removed, and is not a routine clinical choice of orthopaedic surgeons at Capital and Coast District Health Board. There remains the possibility that a fibreglass cast which is lightweight and more flexible than a plaster cast and is not easily removed, may provide and alternative method of immobilisation that can accommodate this IPC/JIT device.

These concerns may provide the focus of a future feasibility study assessing the longer term use of the IPC/JIT system within lower limb casts. This study shows that in a sample of healthy volunteers with their leg placed in a below-knee fibreglass cast, the IPC/JIT system substantially increases PSV in the popliteal vein, and this is similar to the PSV of the same system outside of a lower limb cast. Further studies are indicated to address other issues such as skin and cast integrity with prolonged use, patient tolerability and adherence to treatment in a clinical sample of patients with injuries requiring prolonged lower limb immobilisation. 


\section{Chapter Six. Measurement of venous thromboembolism risk factors within the workforce, sedentary time at work and investigation of a possible intervention to promote venous blood flow of the lower limbs.}

In the last 50 years there have been significant changes in the relative proportions of time spent undertaking physical activity of any sort and sedentary time, particularly in developed countries. Increasing access to television, computers and the internet has resulted in an increased proportion of leisure time spent on sedentary activities (File 2013). Over the same period, the proportion of the labour force in high activity occupations has fallen, while the proportion of people in low activity occupations has remained fairly stable (Brownson et al., 2005). Adverse health outcomes and increased mortality associated with sedentary behaviour have been demonstrated consistently in the medical literature (Dunstan et al., 2010; Katzmarzyk et al., 2009; Koster et al., 2012; Matthews et al., 2012; Alpa V. Patel et al., 2010).

While many of these studies have focused on cardiovascular disease, cancer risk and at times, respiratory illness, they have also reported on all-cause mortality, of which VTE mortality is a subset. Even in these large population studies the frequency of VTE as a fatal event, the lack of autopsies at death and variable death certificate documentation would make it very difficult to quantify the proportion of fatalities attributable to VTE.

In the last decade there has been increasing recognition of the contribution of sedentary behaviours to adverse health outcomes, such that the member states of the World Health Organisation agreed to a target of reducing insufficient physical activity by $10 \%$ by 2020 , as sedentary behaviour is now regarded as a key modifiable risk factor in the prevention of non-communicable diseases (World Health Organisation (WHO), 2013).

Reports in the medical literature of VTE associated with prolonged periods of occupational and / or computer-related sitting began in the early 2000's with a number of case reports of otherwise healthy individuals presenting with VTE events and no other apparent cause 
other than one or more episodes of prolonged sitting immediately prior to their VTE event (Beasley et al., 2005; Beasley, Raymond, Hill, Nowitz, \& Hughes, 2003; Chew, 2006; Hughes et al., 2003; H. Lee, 2004; K. Lee et al., 2015). West and colleagues published the first casecontrol study investigating the association between prolonged seated immobility at work and VTE (West et al. 2008). There was an association between the risk of VTE and maximum hours seated at work in a 24 hour period, and maximum hours seated without getting up, consistent with studies of VTE and travel-related risk (Lapostolle et al., 2001; Paganin et al., 2003). In a second case-control study Healy and colleagues reported a 2.8-fold increased risk of VTE with prolonged work- and computer-related immobility (B. Healy, Levin, et al., 2010), and a $10 \%$ increased risk of VTE with each hour increase in maximum sitting time and with each hour increase in average sitting time in a 24 hour period.

Similar to Healy and colleagues, I demonstrated in Study One that each one hour increase in mean hours seated within a 24 hour period was associated with an $8 \%$ increased risk of VTE and that there was a weak association between prolonged work- and computer-related seated immobility and VTE risk. In Study Two, I showed there was a significant association between sedentary occupations and VTE with a $79 \%$ increased risk of VTE $(P=0.003)$. It seems increasingly clear, that along with increased risk of poor health outcomes, sedentary occupations are associated specifically with an increased risk of VTE. Although the absolute numbers that might be affected by this risk are quite small, the number of individuals at risk is high, and sedentary behaviours represent a modifiable risk factor for VTE. There remains a question of how to modify these behaviours in the workforce in a cost effective way that does not negatively impact productivity.

Interventions to improve employee activity in the workplace have been attempted, but most have tended to have transient effects and to be resource intensive (Dugdill et al., 2008). Any interventions also need to have minimal impact on worker productivity. Recent studies of 'walking workstations' which allow workers to walk while they work have been found to have an initial negative effect on productivity with reduced computer task performance and loss of fine motor control of the computer mouse (John, Bassett, Thompson, Fairbrother, \& Baldwin, 2009; Straker et al., 2009; Thompson et al., 2008). 
I have shown in Study Four that the Legflow device improves lower limb haemodynamics in adults seated in office chairs, with a 10 to 11 -fold increase in PSV in the popliteal vein. This may provide a minimally intrusive and cost effective option for the mitigation of venous stasis during prolonged sitting in the workplace, thereby reducing the risk of VTE. While the haemodynamic effect of the Legflow has been demonstrated, the feasibility of the Legflow device in an office environment has yet to be assessed.

This cohort study of a convenience sample of 96 adult office workers who are classified as sedentary according to the ANZSCO criteria has been designed to assess the use of the Legflow in a regular office environment. Participants were asked to use the Legflow device once every hour while they were at work (between seven and eight times per day). I defined 'adequate adherence' as $50 \%$, i.e. participants would have to use the Legflow device four times a day or more. Therefore the primary outcome variable was the proportion of participants that used the Legflow device an average of four times or more per day during the study period.

This study allowed an assessment of underlying VTE risk in the participants. By using similar risk factor assessment methodology to that already been used in previous case-control studies of work- and computer-related immobility (I Braithwaite, Maher, et al., 2016; B. Healy, Levin, et al., 2010; West et al., 2008), I had an opportunity to understand pre-existing risk factors for VTE in the sample that participate in the study, and the ability to directly compare some of the work-related sitting parameters to those of previous studies.

At the same time I took the opportunity to explore worker perception of their work-related sedentary activity and to compare this to an objective measure of the same.

Finally, not only is it important that a preventive device is actually used, but it must not be noxious to the users, therefore I also sought qualitative feedback on the acceptability of the Legflow device and the likelihood of its continued use in a sedentary workplace environment by the participants.

To ensure that participants remained focused on documentation of Legflow usage and were not encouraged to change their behaviour by measuring their own activity levels, I decided 
to utilise 'off the shelf' pedometers $\left(\right.$ Fitbit ${ }^{\circledast}$ Flex $^{T M}$ ) to measure participant activity rather than have them document the activity themselves. This also allowed an assessment of the difference between participant-reported work-related sitting behaviours and objectively measured activities. However, for the Fitbit $^{\circledR}$ Flex $^{T M}$ to be a useful tool in this respect it needed to be validated for the physical activity being measured and its limits of agreement calculated. 


\section{Validation of the Fitbit ${ }^{\circledR}$ Flex $^{T M}$ to measure the sedentary time during work regarding the 'Dome device at work study'}

The secondary outcomes of Study Seven are associated with capturing worker activity in their usual workplace by having them wear a simple 'off the shelf' pedometer. To measure this variable, I elected to use the Fitbit ${ }^{\oplus}$ Flex $^{T M}$ due to consumer familiarity and acceptance of the product, the ease of wearing the device, the ability to wear the device in multiple situations to ensure data gathering is ongoing throughout the week, a battery life of between 5 and 10 days depending on user activity and the ability to avoid recall bias through participants documenting seated and active times in a handwritten diary.

The device Fitbit ${ }^{\circledR}$ Flex $^{T M}$ tracks all steps completed by participants, can be worn on the wrist or the ankle and summarizes the values in five minute periods on the Fitbit ${ }^{\circledast}$ application which can be downloaded onto a smart phone or a computer. It is also possible to access Fitabase $^{\circledast}$, a cloud based Fitbit ${ }^{\circledast}$ data management system, which enables researchers to access data in one minute increments and download that data into CVS or Excel formats for ready manipulation.

The Fitbit $^{\circledR}$ Flex $^{\text {TM }}$ trackers use a three-axis accelerometer to measure movement (Fitbit, 2016). An accelerometer is a device that turns movement of a body into digital measurements when attached to the body. By analysing acceleration data, the devices provide information about frequency, duration, intensity, and patterns of movement to determine the number of steps taken over time. The three-axis programme allows the accelerometer to measure movement in any direction. The Fitbit ${ }^{\circledR}$ Flex $^{T M}$ trackers have an algorithm for step counting (Fitbit, 2016). The algorithm is designed to look for motion patterns most indicative of people walking. The algorithm has an inbuilt threshold to try and ensure that a measured step is in fact a step. If a movement and its associated acceleration measurement data meet the threshold, the motion will be counted as a step. If that threshold is not met, the algorithm will not count the motion as a step. There are instances where there can be enough acceleration without movement to exceed the threshold and therefore cause some over counting of steps, such as clapping hands. Equally, it's possible 
for the algorithm to undercount (not meet the required acceleration threshold), for example by tip-toeing or walking on very soft surfaces. As well as the inherent limits of over- or under-counting steps, the use of the Fitbit ${ }^{\circledR}$ Flex $^{T M}$ is limited in research studies, so I undertook a comparison of Fitbit ${ }^{\circledR}$ Flex ${ }^{T M}$ measured data compared to volunteer collected diary data of sedentary and active time to assess the likely variance between volunteer documented activity and that collected by the Fitbit $^{\circledast}$ Flex $^{T M}$.

\section{Methods: validation study}

The Fitbit ${ }^{\circledR}$ Flex $^{T M}$ was tested on 10 different adult volunteers who comprised a convenience sample from the Medical Research Institute of New Zealand. Before use, each separate Fitbit $^{\circledR}$ Flex $^{T M}$ (wireless activity and sleep wristband) was installed under a unique study ID on the Fitabase ${ }^{\circledR}$ software on a PC. The installation included date/time stamp capacity for the New Zealand Time Zone $(G M T+12)$. The Fitbit ${ }^{\circledR}$ Flex $^{T M}$ pedometer comes with a pre-set daily step goal of 10,000 steps. This goal was reset to 100,000 steps on all pedometers to ensure that the total steps per day remained blinded to participants and there would be no incentive to set step targets during the day. The ID of the Fitbit flex was noted for each participant. All participants wore the Fitbit $^{\circledR}{ }^{\text {Flex }}{ }^{T M}$ (figure 6.1) during working hours on their wrist.

At the same time as wearing the Fitbit ${ }^{\circledR}$ Flex $^{T M}$ they were asked to write down their seated and unseated times in a diary while they were at work. The diary comprised pre-determined 5 minute increments, starting from 8am in which participants were asked to denote whether they were sitting or otherwise. Participants were asked to synchronise their watches to the Fitbit PC application, to ensure as much as possible that the times they recorded would match with those steps recorded by the Fitbit ${ }^{\circledR}$ Flex $^{T M}$. After one work day of recording activity, each Fitbit ${ }^{\circledR}$ Flex $^{T M}$ was synchronised to the Fitabase ${ }^{\circledR}$ software (by Bluetooth or charger) to show the counted steps in five minute periods and the data relating to that participant was downloaded onto an Excel file. Through Fitabase ${ }^{\circledR}$, steps accrued for each minute the device was worn, were downloaded onto an excel spreadsheet. The data was 'cut' so that steps were analysed only for the minutes that each participant had 
indicated they were wearing the Fitbit $^{\circledast}$ Flex $^{\text {TM }}$ while at work, e.g. 8.15 am to 5.30 pm, and the remaining data were discarded.

The Fitbit $^{\circledR}$ Flex $^{\text {TM }}$ internal programme designates less than 25 steps in each five minute period as 'sedentary time' which roughly equates to a metabolic equivalent of task (MET) of one to 1.5. One MET is equal to the rate of energy produced per unit surface area of an average person seated at rest and 1.5 Mets is equivalent to sitting at a desk typing, writing or other desk work. The five minute periods categorised as 'sedentary time' from the Fitbit ${ }^{\circledR}$ Flex ${ }^{T M}$ were compared with the seated and unseated times noted by the participants in their diary while wearing the Fitbit ${ }^{\circledR}$ Flex $^{T M}$.

The mean difference and the limits of agreement between the two methods of measurement were calculated using the Bland-Altman method. In the absence of any previously published data assessing the limits of agreement of reported versus measured seated behaviours, it was deemed that if the limits of agreement between sedentary time as reported by the volunteers and that measured by the Fitbit ${ }^{\circledR}$ Flex $^{T M}$ were within five minutes for every accrued sedentary hour, this would be acceptable for the study.

Figure 6.1: Fitbit ${ }^{\circledR}$ Flex $^{\mathrm{TM}}$ as usually worn by a participant

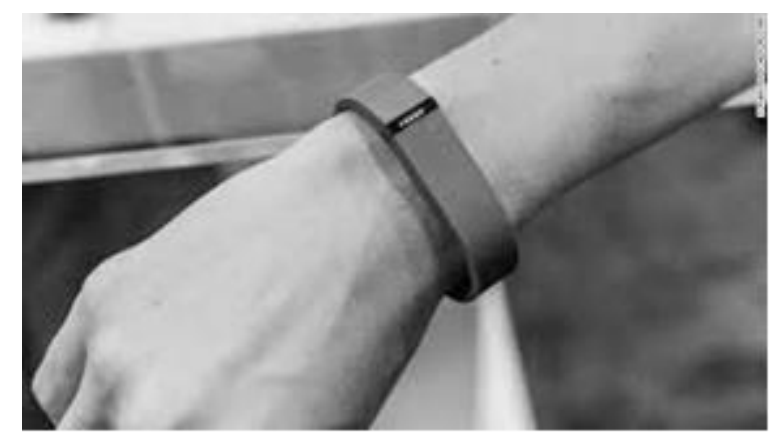




\section{Results: validation study}

The summarised data for the participants is shown in Table 6.1. The mean reported sitting time per volunteer was 296.3 minutes and the mean Fitbit $^{\circledR}$ Flex $^{T M}$ measured sitting time was 284.5 minutes. The mean (range) difference between Fitbit $^{\circledR}$ Flex $^{\text {TM }}$ reported sitting time and volunteer-reported sitting time was $-2.07 \%(95 \% \mathrm{Cl}-9.7 \%$ to $6.0 \%)$. The BlandAltman limits of agreement between the Fitbit Flex and volunteer-reported sitting time was $-11.8(95 \% \mathrm{Cl}-40.74$ to 17.14$)$, indicating that by comparison with volunteer diaries, the Fitbit ${ }^{\circledR}$ Flex $^{T M}$ under-reported sitting times by approximately 12 minutes, an average 2.4 minutes for each accrued volunteer-reported sitting hour. I deemed this to be satisfactory for the study. 
Table 6.1: Comparison of Fitbit $^{\circledR}$ Flex $^{T M}$ recorded time in five minute increment and documented active/ sedentary time by participants.

\begin{tabular}{|c|c|c|c|c|}
\hline & $\begin{array}{l}\text { Fitbit }^{\circledast} \\
\text { Flex }^{T M} \\
\text { placed }\end{array}$ & $\begin{array}{l}\text { Sedentary time } \text { Fitbit }^{\circledR} \\
\text { Flex }^{\top M} \text { (Minutes) }\end{array}$ & $\begin{array}{l}\text { Sedentary time } \\
\text { volunteer diary } \\
\text { (minutes) }\end{array}$ & $\begin{array}{l}\text { Variation (Fitbit }^{\circledR} \\
\text { Flex }^{T M} \text { minus diary) } \\
(\%)\end{array}$ \\
\hline Person 1 & wrist & 240 & 235 & $+2.1 \%$ \\
\hline Person 2 & wrist & 250 & 275 & $+2.0 \%$ \\
\hline Person 3 & wrist & 235 & 240 & -2.1 \\
\hline Person 4 & wrist & 295 & 310 & -4.8 \\
\hline Person 5 & wrist & 265 & 250 & +6.0 \\
\hline Person 6 & wrist & 320 & 335 & -4.4 \\
\hline Person 7 & wrist & 285 & 310 & -8.1 \\
\hline Person 8 & wrist & 285 & 300 & -5.0 \\
\hline Person 9 & wrist & 325 & 360 & -9.7 \\
\hline Person 10 & wrist & 345 & 350 & -1.5 \\
\hline AVERAGE & & 284.5 & 293.5 & -2.07 \\
\hline
\end{tabular}

Individual tables of validation data for each participant are shown in Appendix VII 
This comparison was surprisingly good and likely influenced by three major factors; the volunteers were all employees of a research organisation, and were perhaps more diligent about documenting their sedentary and standing times compared to those with little interest in research, the five minute incremental diary and the five minute MET calculations would have applied a smoothing effect, and finally, asking employees to synchronise watches with the Fitbit ${ }^{\circledast}$ Flex $^{T M}$ timing mechanism would have helped to reduce error associated with poor synchronicity between the volunteers time piece and that of the Fitbit $^{\circledR}$ Flex $^{\text {TM }}$.

\section{Conclusion}

The Fitbit ${ }^{\circledast}$ Flex $^{\text {TM }}$ is an appropriate tool to measure sedentary activity in the workplace of adult participants, and is likely to under-report seated time by approximately 2.4 minutes per accrued sitting hour compared to participant diaries. 
STUDY SEVEN: Measurement of venous thromboembolism risk factors within the workforce, sedentary time at work and investigation of a possible intervention to promote venous blood flow of the lower limbs: (Dome device at Work Study).

\section{Hypothesis}

The hypothesis for this study was that the Legflow device is a feasible workplace intervention for sedentary office staff.

\section{Primary outcome variable}

The primary outcome variable for this study was the proportion of participants that utilised the Legflow device four or more times per day (defined as adequate adherence).

\section{Secondary outcome variables}

Secondary outcome variables were; the proportion of participants that had one or more risk factors for VTE, the proportion of participants that met the criteria for prolonged workrelated sitting, a comparison of participant-reported sitting time and measured sedentary time, and an assessment of participant acceptance of the Legflow device.

\section{Methods}

This was a cohort study of a convenience sample of 96 adults working in roles that would be deemed sedentary according to the Australia New Zealand Standard Classification of Occupation (ANZSCO) codes, utilising the Legflow in the course of their usual working week in their usual working environment.

The proportion of participants who used the Legflow device at least 4 times per day $(50 \%$ adherence) was estimated by the exact binomial method with $95 \%$ confidence intervals. The proportion of participants with one or more risk factors for VTE was calculated and expressed as a percent of the sample. The proportion of participants who reported sitting behaviours consistent with prolonged work-related immobility was calculated and expressed as a percent of the sample. A student t-test was used to assess if there was a difference between sitting hours per day and maximum sitting time reported by the 
participants and the same variables as measured by the Fitbit ${ }^{\circledast}$ Flex $^{T M}$. Ordinal scale variables relating to qualitative feedback about the device were dichotomised into positive (e.g. very comfortable + comfortable) versus negative (e.g. uncomfortable and very uncomfortable), and estimates and confidence intervals were calculated for the proportions. 


\section{Methods}

After gaining permission from employers of office based staff which included negotiating memoranda of understanding, and application for ethics approval at each locality through the Health and Disability Ethics Committee online approval system, I visited each working environment weekly to recruit eight volunteers at a time to trial the Legflow device for one working week. This continued until a total of 96 office workers had been recruited into the study. To be eligible for the study participants had to be aged between 18 and 75 years and employed in a role that was considered sedentary. Convenience samples working in government departments in Wellington New Zealand were approached to participate in the study with the aim of recruiting 100 desk-based workers.

The rationale for government workers was due to the concentration of such workers in the Wellington region, increasing the likelihood that the results would be generalisable to most office based government workers, and potentially to those non-government employees who undertook similar tasks. Te Puni Kokiri (Ministry of Maori Development) was approached and 29 participants were recruited. The Ministry of Social Development Family and Child Services Department yielded another 67 recruits. Recruitment was stopped at 96 as the volunteer pool at both locations was exhausted, and obtaining a contract or memorandum of understanding, and HDEC approval with a further government site in order to recruit four more participants was considered infeasible.

After completing informed consent procedures, participants completed an intervieweradministered questionnaire (Appendix IX) that included demographic data, occupational description, VTE risk factors, office working environment, usual working hours and usual work sitting habits. VTE risk factors were grouped into categories; personal history of VTE, family history of VTE, thrombophilias, pregnancy, medications, recent surgery, recent travel for a period greater than four hours, medical immobility, varicose veins, smoking, active cancers. Participants were asked what their usual daily work hours were, and what proportion of that was spent sitting. They were also asked the maximum numbers of hours they spent sitting in a usual work day, how long they usually sat before they got up to move around, and the maximum time spent sitting before they got up and moved around. 
Participants were each given a Legflow device, and its' use was demonstrated. After the demonstration, participants were asked to demonstrate the use of the Legflow themselves to ensure that they understood how it was to be used. Participants were given a pre-printed diary (Appendix X) in which to record the time of day that that they used the Legflow and how long they used it for during the working week. At the end of the week they were asked to complete a short questionnaire and to rate how convenient they found the device, how easy and comfortable it was to use and how likely they would be to use it regularly throughout their working days in the future. These ratings were measured on a scale of 1 (very convenient / easy / comfortable / likely to use it again) to 4 (very inconvenient / very hard / very comfortable / definitely not likely to use it again). They were asked to note down any adverse events associated with the device in the workplace.

Early on it became apparent that a number of participants were constantly using the Legflow device, and unable to record singular episodes of use, documenting instead 'continuous use' or similar comments. There were also a number of participants who were sick or out of the office for various reasons throughout their study week. Therefore to assess the primary outcome variable the following steps were taken:

- For those who used the Legflow device continuously, the precise number of times used and duration of use could not be determined. For the purposes of analysis, it was assumed that use would be at least four times per day, and as such, for each day the device was used continuously, this would be taken to be a day that met usage criteria, i.e. constant use equals a mean of four times per day.

- For those who did not use the Legflow at all, they were included in an intention to treat analysis (i.e. $\mathrm{N}=95)$.

- For those who whose diaries were not completed for a particular day without an indication of sickness, annual leave or travel away from the office for any reason, this was taken to mean that the Legflow was not used for that day

- For those who noted that they were sick, on annual leave or had travelled away from the office for a work related reason, this day was excluded from the analysis 
- Three participants noted total time used per day without documenting individual episodes, this was conservatively taken to mean a single use only for that day

Fitbit $^{\circledR}$ Flex $^{T M}$ pedometers were each given a unique identification code and password for the Fitabase $^{\circledR}$ (Small Steps Lab LLC, San Diego, Ca, USA) data management system, which the participants did not have access to, ensuring that the participant remained blind to the steps recorded each day. Participants were also advised that the steps per day function on the Fitbit Flex had been removed, so they were unable to track their steps in accordance with the standard package that comes with the Fitbit $^{\circledast}$ Flex $^{\mathrm{TM}}$. Those participants that usually wore their own pedometer were allowed to continue doing so. Participants were each allocated a Fitbit ${ }^{\circledR}$ Flex $^{T M}$ to wear for the same working week that they were using the Legflow device. Where possible they were asked not to remove the Fitbit $^{\circledast}$ Flex $^{T M}$ for the entire week to ensure that no data would be lost due to participants forgetting to put the device on each day.

Participants documented start and finish times for each working day they wore the Fitbit ${ }^{\circledast}$ Flex $^{T M}$, and only data collected by the device during those documented hours was analysed. The Fitbit ${ }^{\circledast}$ Flex $^{T M}$ data was captured in Fitabase ${ }^{\circledR}$ to allow minute by minute analysis of participant steps. In accordance with Fitbit $^{\circledR}$ Flex $^{T M}$ MET algorithms, less than 25 steps per five minute period was considered sedentary activity (METS of 1.5 or less). From this data, mean hours seated during the working day and the maximum period of sitting time without getting up during all measured working hours was calculated. These numbers were compared to participant answers about work sitting habits.

The sample size was calculated on the assumption that there would be a $50 \%$ utilisation of the Legflow device (i.e. it would actually be used four times in an average 7.5 to 8 hour working day throughout the 5 day period). A sample size of 100 had $90 \%$ power and an alpha of 5\% to rule out a proportion adherence below 35\% (i.e. less than 3.2 times / day). For the proportion of individuals who have risk factors for VTE, a sample size of 100 had a margin of error $(95 \% \mathrm{Cl}$ for a proportion of $50 \%)$ of plus or minus $10 \%$. 


\section{Statistical analysis}

The proportion of participants who used the Legflow device at least 4 times per day $(50 \%$ adherence) was estimated by the exact binomial method with $95 \%$ confidence intervals. The proportion of participants with one or more risk factors for VTE was calculated and expressed as a percent of the sample. The proportion of participants who reported sitting behaviours consistent with prolonged work-related immobility was calculated and expressed as a percent of the sample. A student t-test was used to assess if there was a difference between sitting hours per day and maximum sitting time reported by the participants and the same variables as measured by the Fitbit ${ }^{\circledast}$ Flex $^{T M}$. Ordinal scale variables relating to qualitative feedback about the device were dichotomised into positive (e.g. very comfortable + comfortable) versus negative (e.g. uncomfortable and very uncomfortable), and estimates and confidence intervals were developed for the proportions.

Statistical analysis was undertaken on SAS 9.3

Ethics approval was obtained from the New Zealand Health and Disability Committee Southern Committee (15/STH/6) and was prospectively registered with the Australia New Zealand Clinical Trials Registry: ACTRN12614000186651. 


\section{Results}

\section{Participant Characteristics.}

The flow of participants is shown in Figure 6.2 and participant characteristics are shown in Table 6.2.

Figure 6.2: Flow of participants through study

Approached: Family and Children's Services at Ministry of Social Development New Zealand and Te Puni Kokiri, Wellington New Zealand

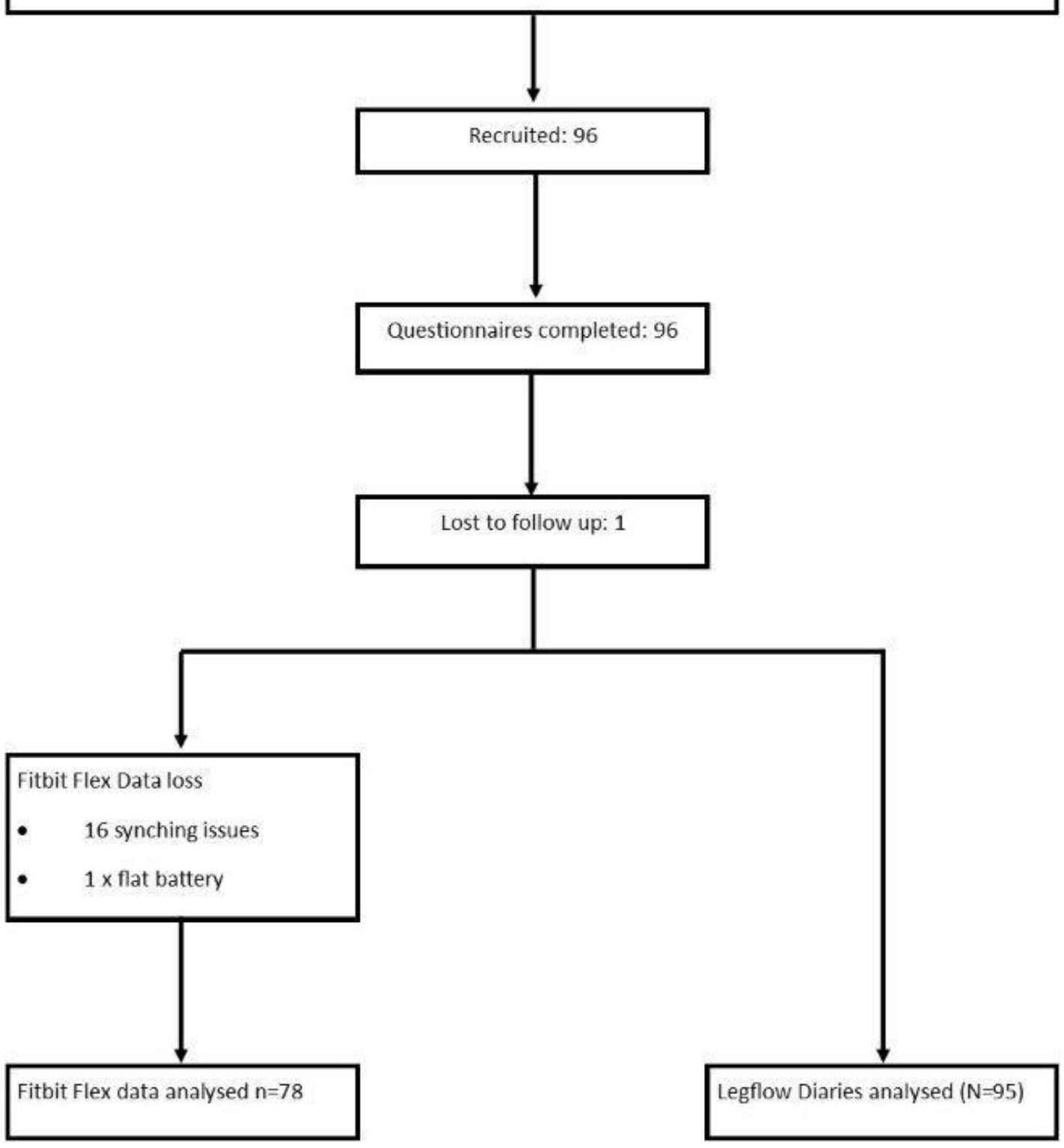


The mean (SD) age of the participants was 43.7 (11.2) years, and the mean (SD) BMI was $28.4(6.3) \mathrm{kg} / \mathrm{m}^{2}$. Females comprised $79 \%$ of the sample, and $58 \%$ were European and $25 \%$ Maori. The most frequently reported risk factors for VTE within this sample were; obesity (24\%), travel greater than four hours duration within the last 28 days (21\%), smoking (15\%), varicose veins $(15 \%)$, family history of VTE $(11.5 \%)$, use of the OCP $(8 \%)$, pregnancy or other hormonal treatments (7\%). There were no reports of surgery within the last 28 days or active cancer. $4.2 \%$ of the participants reported taking aspirin, $100 \mathrm{mg}$ daily. 
Table 6.2: Participant characteristics

\begin{tabular}{|c|c|c|}
\hline & \multicolumn{2}{|c|}{ Mean (SD) } \\
\hline Age & \multicolumn{2}{|c|}{$43.7(11.2)$} \\
\hline \multirow[t]{2}{*}{ BMI $\left(\mathrm{Kg} / \mathrm{m}^{2}\right)(\mathrm{N}=86)$} & \multicolumn{2}{|l|}{$28.4(6.3)$} \\
\hline & $\mathbf{N}(\%)$ & $95 \% \mathrm{Cl}$ \\
\hline Female & $76(79.2)$ & 69.7 to 86.8 \\
\hline European & $56(58.3)$ & 47.8 to 68.3 \\
\hline Maori & $24(25.0)$ & 16.7 to 34.8 \\
\hline Asian & $6(6.3)$ & 2.3 to 13.1 \\
\hline Pacific Island & $4(4.1)$ & 1.1 to 10.3 \\
\hline Other ethnicity & $6(6.3)$ & 2.3 to 13.1 \\
\hline \multicolumn{3}{|c|}{ Risk factors for VTE } \\
\hline Obese $\left(\mathrm{BMI}>30 \mathrm{Kg} / \mathrm{m}^{2}\right)(\mathrm{N}=86)$ & $29(33.7)$ & 23.9 to 44.7 \\
\hline Travel $^{1}$ & $20(21.1)$ & 13.4 to 30.6 \\
\hline Smoking & $14(14.6)$ & 8.2 to 23.3 \\
\hline Varicose veins & $14(14.6)$ & 8.2 to 23.3 \\
\hline Family history VTE & $11(11.5)$ & 5.9 to 19.6 \\
\hline Oral contraceptive pill & $8(8.3)$ & 3.7 to 15.8 \\
\hline Pregnancy / hormonal treatment & $7(7.4)$ & 3.0 to 14.6 \\
\hline Personal history of VTE & $4(4.2)$ & 1.1 to 10.3 \\
\hline Immobility $^{2}$ & $3(3.2)$ & 0.7 to 9.0 \\
\hline Thrombophilia & $3(3.2)$ & 0.7 to 9.0 \\
\hline Lower limb immobilisation ${ }^{3}$ & $1(1.0)$ & 0.03 to 5.7 \\
\hline
\end{tabular}

Work related risk factors for VTE

\begin{tabular}{lcc}
\hline $\mathrm{SIT}^{4}$ at work & $10(10.4)$ & 5.1 to 18.3 \\
Work at home & $32(33.3)$ & 24.0 to 43.7 \\
$\mathrm{SIT}^{4}$ at home & $1(1.0)$ & 0.03 to 5.7
\end{tabular}

1 Travel risk: undertook a journey of greater than four hours in the 28 days prior to answering the questionnaire 2 Immobility: two days of immobility for any reason in the 28 days prior to answering the questionnaire

3 Lower limb immobilised in cast in the 28 days prior to answering the questionnaire

4 SIT - Seated immobility: work related sitting in excess of ten hours within one working day, with a period of at least two hours without getting up and moving around, this episode to have occurred at least once within 28 days of the questionnaire being completed. 


\section{Use of the Legflow device}

One participant answered the questionnaire at one visit, and then realised they were not going to be in the office for the remainder of the week. It was agreed they would use the Leglow and the Fitbit $^{\circledR}$ Flex ${ }^{T M}$ for the following week, however, they failed to turn up despite reminders. This was considered a withdrawal from that time point, so their questionnaire data was retained in the analysis without associated Fitbit $^{\circledR}$ Flex $^{T M}$ and dome data.

For Legflow use, of the remaining 95 participants, 5 (5.2\%) did not use the Legflow at all while they were at work and $63(66.3 \%)$ used it all the days they were at work, comprising one third (21) who used it continuously and two thirds (42) who used the Legflow for discrete episodes (Table 6.3). There were 27 (28.4\%) participants who used the device either constantly or intermittently for some, but not all of the days they were at work.

\section{Participant adherence to Legflow device recommended usage}

The proportion $(95 \% \mathrm{Cl})$ of participants that used the Legflow for 4 or more times per day was $50.5 \%$ (40.1 to $60.9 \%$ ). Of these, 29 used the Legflow episodically and 19 used it constantly.

Table 6.3: Use of the Legflow reported in participant diary

Legflow use, all participants $(\mathrm{N}=95)$

\begin{tabular}{lll}
\hline Usage pattern & $\mathbf{N}(\%)$ & $95 \% \mathrm{Cl}$ \\
\hline Did not use at all & $5(5.2)$ & 1.7 to 11.9 \\
Used every day while at work & $63(66.3)$ & \\
$-\quad$ Continuously used & $21(22.1)$ & 14.2 to 31.8 \\
$-\quad$ Episodic use & $42(44.2)$ & 34.0 to 54.8 \\
Used some of the days at work & $27(28.4)$ & 19.6 to 38.6 \\
Used $\geq$ four times / day & $48(50.5)$ & 40.1 to 60.9 \\
$-\quad$ Continuously used & $19(39.6)$ & 25.8 to 54.7 \\
$\quad-\quad$ Episodic use & $29(60.4)$ & 45.3 to 74.2 \\
\hline Cl - Confidence interval & &
\end{tabular}


Risk factors for VTE without prolonged work-related immobility

Not including prolonged work-related immobility, 26 (27.1\%) participants had no risk factors for VTE, 37 participants (38.5\%) reported one risk factor for VTE, $16(16.7 \%)$ reported two risk factors for VTE, 15 (15.6\%) reported three risk factors and two (2.1\%) reported four risk factors for VTE. The mean (SD) number of risk factors reported was 1.3 (1.1) (Figure 6.3).

Figure 6.3: Frequency of pre-existing risk factors for venous thromboembolism reported by participants (exclusive of prolonged work-related seated immobility).

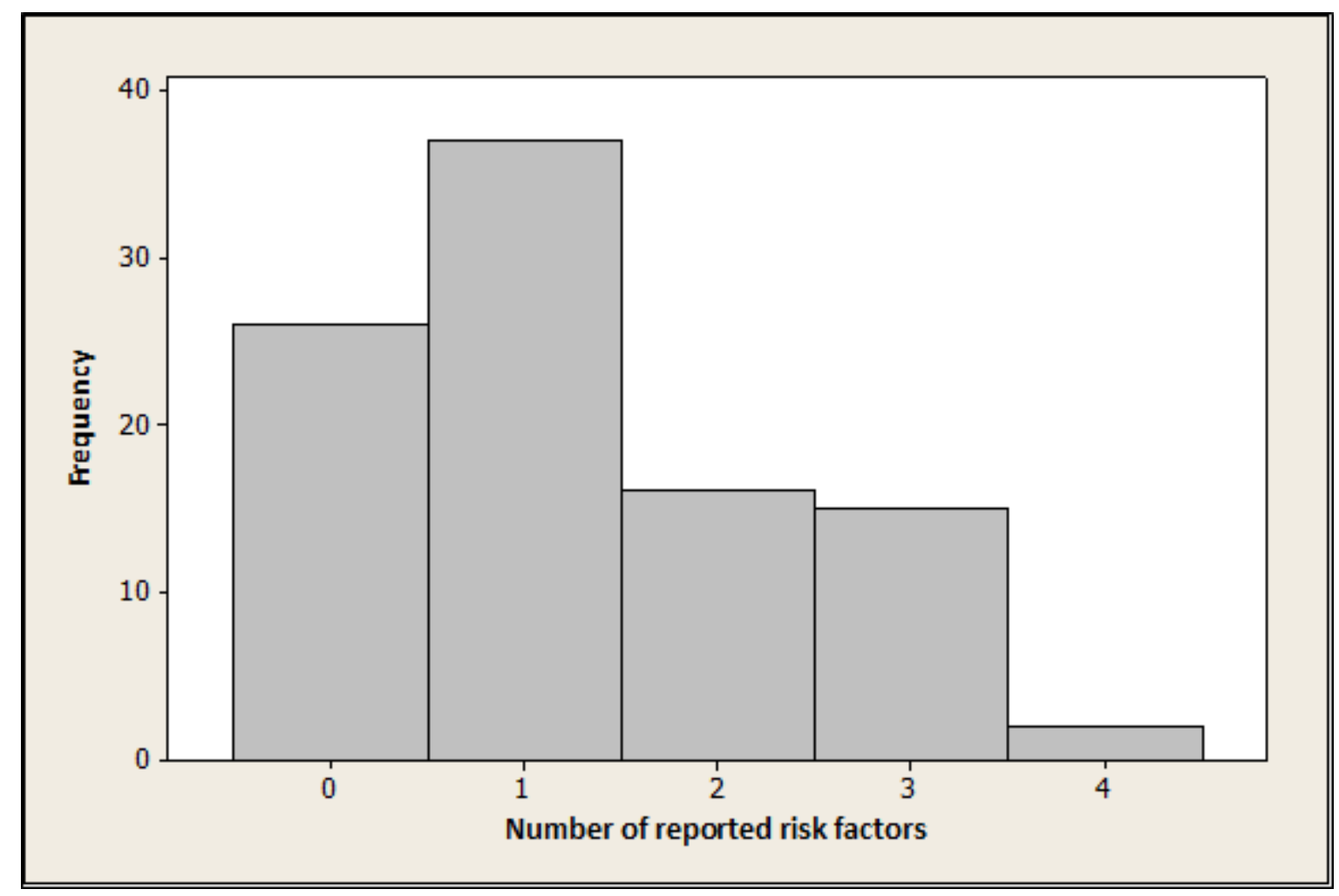


Risk factors for VTE including work-related immobility

When prolonged work-related seated immobility as reported by the participants was considered, 25 (26.0\%) participants had no risk factors for VTE, 34 (35.4\%) participants reported one risk factor for VTE, 16 (16.7\%) reported two risk factors for VTE, 17 (17.7\%) reported three risk factors for VTE and four (4.2\%) reported four risk factors for VTE, with a mean (SD) of 1.4 (1.2) risk factors (Figure 6.4).

Figure 6.4: Frequency of risk factors for venous thromboembolism reported by participants inclusive of prolonged work-related seated immobility.

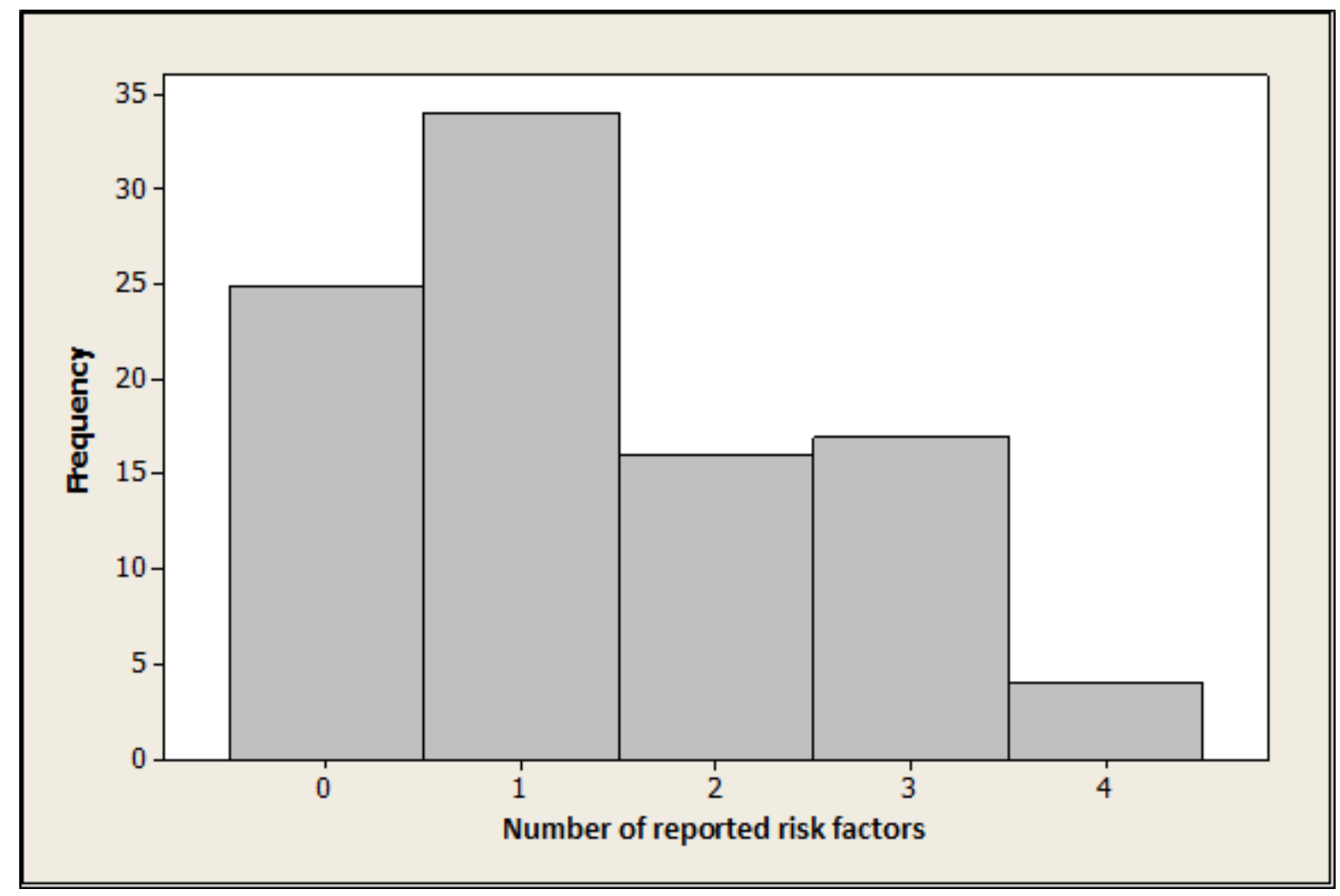




\section{Reported sitting habits}

All 96 participants completed the questionnaire about work-related sitting habits during the working day. By participant recall, there was a mean (SD) of 8.2 (0.8) hours worked in a usual working day (Table 6.4). Participants estimated they spent 6.2 hours (75\%) of that time seated. The mean (SD) longest hours worked were $9.6(2.0)$ hours in a usual working day, and the mean (SD) longest hours seated were $7.6(2.1)$ hours in a working day. With respect to the usual sitting time without getting up, the reported mean (SD) was $1.2(0.7)$ hours and the maximum sitting time without getting up was 2.3 (1.2) hours.

One third (32/96) of participants reported undertaking work-related activities on the computer at home.

Table 6.4: Table of work-related sitting parameters reported by participants

Participant reported work-related sitting parameters ( $N=96$ ) Mean (SD) $\quad 95 \% \mathrm{Cl}$

Days worked per week $4.9(0.3) \quad 3$ to 5

Usual hours in a working day $8.2(0.8) \quad 6.0$ to 10.5

Maximum hours worked in one day

$9.6(2.0) \quad 7.0$ to 20

Usual sitting time in a working day

$6.2(1.1) \quad 3.5$ to 9.5

Maximum sitting time in one day

$7.6(2.1) \quad 3.5$ to 18

Usual sitting time without getting up

$1.2(0.7) \quad 0.1$ to 3.0

Maximum sitting time without getting up

2.3

0.5 to 7

SD - Standard deviation

$\mathrm{Cl}$-Confidence interval 


\section{Proportion of participants meeting the criteria for prolonged work-related immobility}

There were ten participants that met the criteria for prolonged work-related immobility (ten hours or more sitting in one day and at least 2 hours at a time without getting up) while at work within the 28 days prior to answering the questionnaire and an additional participant met the criteria for prolonged work-related immobility while working at home. The proportion $(95 \% \mathrm{Cl})$ of participants meeting the criteria for prolonged work-related seated immobility was $11 \%$ (4.7 to $17.3 \%)$.

\section{Fitbit $^{\circledR}$ Flex $^{T M}$ recorded activity.}

There was initial difficulty downloading the Fitbit ${ }^{\circledast}$ Flex $^{T M}$ data due to not synchronising the Fitbit ${ }^{\circledast}$ Flex $^{T M}$ devices with the Fitbit ${ }^{\circledR}$ Flex $^{T M}$ and Fitabase ${ }^{\circledR}$ applications frequently enough and immediately prior to randomisation to the participants. This affected 16 participants in total, early in the study. One participant failed to hand in their device before the battery was fully flat and their data was also unable to be retrieved. Taking into account the participant that withdrew from the study, Fitbit ${ }^{\circledR}$ Flex $^{\text {TM }}$ data was available for 78 participants. The 78 participants were monitored for a total of 2,896.5 hours (average 37.1 hours each). There were a total of 1,947 sitting hours and 949 active hours. On average, sitting comprised $67 \%$ (range $34 \%$ to $84 \%$ ) of the total time monitored.

The estimated mean (SD) of measured sitting time was 25.0 (6.6) hours and the estimated mean (SD) non-sitting time was 12.2 (4.7) hours (Table 6.5). Participants were sitting for an average of 5.4 hours (range 2.7 to 8.4 ) for each working day that they wore the Fitbit $^{\circledast}$ Flex $^{T M}$. The estimated mean (SD) longest period of non-sitting was $0.9(0.4)$ hours. The monitored steps for each working day are reported in Table 6.5. 
Table 6.5: Fitbit monitored work-related sitting and non-sitting times

\begin{tabular}{lll}
\hline Fitbit $^{\Phi}$ Flex $^{\text {TM }}$ monitored work-related activity (N=78) & Mean (SD) & $\mathbf{9 5 \% ~ C l}$ \\
\hline Total sitting time over the period of time monitored (hours) & $25.0(6.6)$ & 5.5 to 42.0 \\
Longest period seated over period of time monitored (hours) & $2.0(1.0)$ & 0.58 to 7.17$)$ \\
Total non-sitting time over the period of time monitored (hours) & $12.2(4.7)$ & 3.2 to 26.1 \\
Longest period not seated over period of time monitored (hours) & $0.9(0.4)$ & 0.25 to 2.58 \\
Total steps recorded Monday ( $\mathrm{N}=76)$ & $3,175(2,051)$ & 461 to 9,705 \\
Total steps recorded Tuesday (N=73) & $4,369(2,064)$ & 905 to 11,554 \\
Total steps recorded Wednesday (N=73) & $4,314(2,060)$ & 415 to 11,228 \\
Total steps recorded Thursday (N=71) & $4,204(2,161)$ & 169 to 12,457 \\
Total steps recorded Friday (N=68) & $4,484(2,558)$ & 854 to 15,073 \\
Total steps for week & $18,957(8,023)$ & 4,105 to 42,686
\end{tabular}

SD - Standard deviation

$\mathrm{Cl}$ - Confidence interval 


\section{Comparison of participant-reported sitting time and monitored sitting time}

In the 78 participants that provided both questionnaire data related to sitting at work, and for whom there was valid Fitbit ${ }^{\circledR}$ Flex ${ }^{T M}$ data, the difference $(95 \% \mathrm{Cl})$ between reported and measured daily sitting hours was 0.75 hours ( 0.50 to 1.00 hour, $P<0.001$ ) (Table 6.6). The difference between reported and measured maximum sitting time without getting up was 0.47 hours ( 0.19 to 0.75 hours, $P=0.001$ ), indicating that participants tended to over-report their sitting time at work.

Table 6.6: Comparison of participant reported sitting time while at work and monitored sitting activity

$\begin{array}{cccc}\text { Participant } & \text { Fitbit }^{\oplus} \text { Flex }^{\mathrm{TM}} & \text { Participant } & 95 \% \mathrm{Cl} \text { for } \\ \text { reported } & \text { measured } & \text { reported minus } & \begin{array}{c}\text { difference in } \\ \text { percentages }\end{array} \\ (\mathrm{N}=78) & (\mathrm{N}=78) & \text { measured } & \text { (P value) }\end{array}$

Mean (SD) sitting time in hours during usual working day

$5.4(1.0)$

0.75

0.50 to 1.00 $(<0.001)$

Maximum mean sitting time (hours) 2.4 (1.3) $2.0(1.0)$ 0.5 


\section{Participant acceptance of Legflow device}

Of the 96 participants, 73 answered the end of study questionnaire asking them about their attitudes towards the Legflow. When asked about the convenience of using the Legflow at work, $75 \%(95 \% \mathrm{Cl}, 63.9$ to $84.7 \%)$ rated the Legflow as convenient to very convenient. Over $90 \%$ rated the Legflow as easy to use, while $75 \%$ found it comfortable. When asked about the likelihood of using the Legflow at work regularly, $24 \%(95 \% \mathrm{Cl}, 15$ to $36 \%)$ said they would be very likely to use it, and $32 \%$ ( $95 \% \mathrm{Cl}, 21.0$ to $45 \%$ ) said they would be likely to use it, while $31 \%$ said they would be unlikely and $13 \%$ said they would be very unlikely to use it. When asked to consider the possible use of Legflow on an airplane, $27 \%$ and $26 \%$ said they would be very likely and likely to use it respectively.

Suggestions for improvement included making the Legflow wider so that a user could simultaneously exercise both feet comfortably $(n=15)$, making the Legflow heavier and / or improving the capacity for it to grip the floor to prevent it moving around while being used $(n=7)$ and that they would have used it more if it came with some kind of reminder system $(n=5)$. The most common positive feedback included that it was easy $(n=14)$ and comfortable to use $(n=9)$, was a good size $(n=8)$, that it reminded them to be more active $(n=5)$ and that it helped with stretching $(n=2)$.

\section{Adverse events}

With the exception of the person who left inadvertently left their Fitbit ${ }^{\circledR}$ Flex $^{T M}$ in the staff soft drink dispensing machine for an unspecified period of time, there were no adverse events associated with either the use of the Legflow or the Fitbit ${ }^{\circledR}$ Flex $^{T M}$ devices. 


\section{Discussion}

In this feasibility study, $50 \%$ of participants used the Legflow for a minimum of four times per day, indicating satisfactory adherence. There were $11(11.5 \%)$ workers who met the criteria for prolonged seated immobility at work, which has been associated with an increased risk of VTE. Workers over-reported their sitting times by approximately 45 minutes per day. Just over $50 \%$ of the participants said they would be likely to use the Legflow again at work, and a similar proportion indicated they would be likely to use it on a plane.

There are a number of methodological issues that should be noted when interpreting the results of this study. Although participants were asked to fill in their episodes of Legflow use in 'real time' while they were at work, it is possible that a number of diaries were filled in retrospectively and there may be a degree of recall bias. It is uncertain what effect if any, this might have had on the documented episodes of Legflow use. We did not have a device that could effectively measure time and duration of Legflow use, so do not have an objective comparison to the participant's diaries. That five participants reported no use at all of the Legflow is reassuring, as it indicates that participants understood that I wanted to measure actual use and were comfortable to report that they hadn't used it at all or had used it very infrequently.

Early on in the study participants started reporting that they found themselves constantly using the Legflow device and they could not in all honesty give times and durations for each use. In the consequent analysis, these participants were allocated a nominal mean use of four times per day for each day they indicated constant use. This was to ensure that each of them were counted as meeting the requirement for the primary outcome variable, but would have been a clear under-estimation of the actual number of times the Legflow was used for that day. This affected 104 (22\%) of the 475 days included in the analysis.

Participant-reported sitting times were based on participant recall of the previous four weeks of work-related sitting behaviours while participants were conscious that their activity was being monitored while they were wearing the Fitbit ${ }^{\circledast}$ Flex $^{T M}$ and they could well have changed their activity levels leading to less sitting time recorded over that week 
leading to a disparity between reported and measured sedentary times. In this study, although the differences between participant-reported activity and measured activity were significant, they were small, with participants over-reporting daily sitting time and maximum sitting time without getting up by 45 minutes and 30 minutes respectively.

Half the participants used the Legflow four or more times per day, and were more inclined to use it episodically than constantly. There is limited clinical evidence to inform the frequency with which a device like the Legflow should be used. The Thrombosis Charity UK advises that sitting for 90 minutes at a desk causes a $50 \%$ reduction in blood flow of the popliteal vein (Clarkson, 2011), and Hitos has demonstrated that after 100 minutes of sitting blood flow is reduced by $41 \%$ (Hitos et al., 2007). Compared to lying prone, sitting at a desk with knees flexed at 90 degrees and / or 120 degrees for as little as five minutes results in a reduction in popliteal peak systolic velocity of 80 to $85 \%$ (Levin et al., 2008). It would seem reasonable then to try and intervene regularly enough to avoid profound reductions in blood flow, but not so frequently that it would interfere with work-related activities or become onerous to the participants. The measured maximum sitting time without getting up over the study period was 2 hours, therefore hourly use of the Legflow might potentially halve that period of reduced blood flow.

Use of the Legflow results in a 12-fold increase in popliteal vein PSV compared to an immobile leg after sitting in an office chair for 20 minutes (I Braithwaite, Mackintosh, et al., 2016) (Chapter Four). Therefore one might anticipate that the participants who reported constant use in their diaries would be mitigating reduced venous flow the most. Whether the PSV seen in the haemodynamic study of the Legflow was consistently achieved by these participants is in question, as it is unlikely that the foot motion of constant use would be the same as the considered foot movement used in the haemodynamic study, and the degree of plantar venous emptying and calf muscle contraction may have been less than that seen under test conditions.

Inclusive of prolonged seated immobility for work, the 96 participants in this study had an average of 1.4 risk factors for VTE. In total, 71 (74\%) of the participants had one or more risk factors for VTE, and four workers had four risk factors for VTE. Although the background risk 
of VTE in the population is low, with an estimated rate of up to three VTE events per thousand person years (Coon et al., 1973), the risk of VTE events in this sample is likely to be higher given the prevalence of pre-existing risk factors. When prolonged seated immobility at work was removed from the assessment of risk factors, the mean number of risk factors was 1.3, there were 70 people who had one or more risk factors for VTE (a reduction of one), and two who had four risk factors (a reduction of two). This indicates that removing or reducing prolonged seated immobility in the workplace could have a very real effect, not only on those with few risk factors through eliminating risk altogether, but also by reducing an accumulation of risk in those who already have a burden of other pre-existing risk factors.

In this sample, $11 \%$ of participants met the criteria for prolonged work- and computerrelated immobility solely while they were undertaking work related activities. As this is the first study that has specifically measured this variable in a sample of office workers, no comparison of this proportion can be made. Despite any limitations previously discussed, these findings are likely to be generalizable to at least government department office staff, and potentially any staff member in a sedentary role both here and overseas. In March 2016 , a total of 120,000 New Zealanders were employed in the roles of 'Public Administration and Safety' (Statistics New Zealand, 2016), which considers local and central government administrative work roles only. There were an additional 800,000 in Professional scientific and administrative support roles, Education and training roles, Financial and insurance roles and Healthcare and social assistance roles. While it is not clear what proportion of these staff are office-based or sedentary, it is clear that a significant number of employees will be exposed to prolonged work- and computer-related immobility in New Zealand alone.

The finding that this sample sat for approximately two thirds of their working day is consistent with previous studies. Cleme and colleagues reported a study of 72 office workers who wore pedometers for one week and who documented their steps and sitting times during work and outside work, finding that $65 \%$ of their time at work was spent sitting (Clemes, Patel, Mahon, \& Griffiths, 2014). In a smaller study Waters and colleagues reported 
that participants who worked approximately 9.5 hours per day spent just over three quarters of their time sitting according to both participant self-report and accelerometer measurement (Waters et al., 2016). They also found that sitting at work accounted for almost two thirds of total daily sitting. Of concern, it has also been found that prolonged sitting time at work is not usually compensated for by additional physical activity outside of work (Clemes et al., 2014; Jans et al., 2007; Waters et al., 2016).

The mean time sitting during a working day reported by the participants in this study was higher than that seen in the cases and controls assessed in Study One; 6.2 hours versus 5.5 and 4.6 hours respectively. This is not surprising as the VTE cases in Study One were individuals who had suffered a VTE regardless of profession, whereas the participants in this study were deliberately chosen because of their assumed sedentary roles. Given that increasing mean hours seated is associated with an increased risk of VTE (B. Healy, Levin, et al., 2010), the total number of hours sitting at work in this particular sample is of concern. It should also be noted that the reported sitting times include only work-related sitting, so any recreational sitting would be additional to this.

The disparity between reported and measured sitting times may be due to a number of reasons. Firstly, as discussed in methodological issues, the participant-reported sitting times were based on retrospective recall while participants could well have changed their activity levels while they were wearing the Fitbit $^{\circledR}$ Flex $^{T M}$. It is also probable that in answering questionnaires, participants have tended to focus on large blocks of time, whereas pedometers will be capturing all incidental activity throughout the day. Such disparities between self-reported activities and objectively measured movement have previously been reported; in a study of 320 office workers who answered a questionnaire about their physical activity over a one week period, there was no correlation between reported activity and that measured by pedometer (Sitthipornvorakul, Janwantanakul, \& van der Beek, 2014). Similar to this study, participants had under-reported their physical activity.

It is reassuring that participants have over-estimated rather than underestimated their sitting time, as this might be a protective behaviour when trying to reduce sedentary behaviour in the workforce. However, it is of concern that the objectively measured mean 
longest sitting period without getting up was still 2 hours, the period considered important in the definition of prolonged seated immobility. This suggests that the Legflow or other similar intervention has an important potential role to play in alleviating the haemodynamic effects of seated immobility in this kind of working environment.

While only three quarters of the participants elected to provide qualitative feedback on the Legflow device in the end-of-study questionnaire, the feedback was generally quite positive. Over half of the participants indicated they would be very likely or likely to use the Legflow at work and that they found it easy and comfortable to use. A similar proportion advised they would be likely to use the Legflow while undertaking a plane journey. This indicates that if employers were to provide this kind of intervention in the workplace, it would be likely to be used by at least half of their workforce. Some slight modifications to the Legflow, including making the domes slightly wider apart, using a base material that would give it more purchase on the ground and possibly an application or reminder system to use the Legflow may improve the utilisation rates.

In conclusion, the Legflow is a feasible, safe and unobtrusive tool for improving venous haemodynamics in sedentary office workers, with $50 \%$ of office workers using it four or more times per day. Approximately three quarters of the sedentary work force are likely to have one or more risk factors for VTE, they are likely to be seated for approximately $75 \%$ of their working time and one in ten office workers are likely to meet the criteria for prolonged work-related sitting. The Legflow was generally well accepted with over half of respondents indicating they would be likely to use it again, and that they might use in other high risk situations such as prolonged air travel. With the proven haemodynamic effect of the Legflow, good participant uptake and frequency of use in the office environment, the Legflow lends itself well to reducing the VTE risk associated with protracted seated immobility in the sedentary work environment. 


\section{Chapter Seven. A cohort feasibility study of an intermittent pneumatic compression device within a below-knee cast for the prevention of venous thromboembolism}

In Study Three I found that one in every seven VTE patients seen in VTE outpatient clinics at Wellington Hospital reported a period of lower limb immobilisation in the four weeks prior to their index event. Lower limb immobilisation was associated with a 73-times increased risk of VTE and represented the most common preventable risk factor for VTE in this patient group with one in every seven patients reporting a history of lower limb immobilisation within four weeks of their VTE event.

In Study Five, I assessed VTE rates in a cohort of patients with lower limb immobilisation for Achilles tendon injuries after the introduction of aspirin $100 \mathrm{mg}$ daily for routine prophylaxis of VTE risk in this clinical setting. A total of $93 \%$ of patients were prescribed aspirin, compared to the previous practice of prescribing aspirin only to those considered to be of moderate risk of developing VTE, and $6.4 \%$ of patients (all of whom had been prescribed aspirin), developed symptomatic radiologically confirmed VTE. The VTE incidence was similar to the $6.3 \%$ identified in a previous audit where it was not routine to prescribe aspirin (B. Healy, Beasley, et al., 2010) and is consistent with rates of symptomatic VTE in patients who have not received VTE prophylaxis that have been reported in the medical literature (Meek \& Tong, 2012; Saragas \& Ferrao, 2011).

Thus it seems that despite the routine prescription of aspirin to patients with Achilles tendon injuries undergoing lower limb immobilisation, there remains a high risk of symptomatic VTE. While randomised controlled trials of other chemoprophylactic agents such may be indicated, the use of medications in such large cohorts of patients raises the issue of adverse bleeding events, an adverse outcome that could be avoided with the use of an IPC device.

The most appropriate way to understand the relative benefits and risks of chemoprophylaxis and / or mechanical prophylaxis for VTE in this setting would to design 
and undertake a three-arm, parallel groups, randomised controlled trial (proposed RCT) comprising of;

- the current usual preventive treatment of low dose aspirin (100mg daily),

- $\quad$ an intermittent pneumatic compression (IPC) device plus low dose aspirin (100mg daily),

- or rivaroxaban (10mg daily) alone to prevent venous thromboembolism (VTE) secondary to cast immobility for a ruptured Achilles tendon or ankle fracture.

In Study Six, I demonstrated that an IPC device (the IPC/JIT system) is not haemodynamically impaired by its placement within a lower limb fibreglass cast and that an IPC device such as the JIT system might provide a non-pharmacological means to reduce the risk of VTE in the setting of lower limb immobilisation without leading to increased bleeding risk. However, at the time I was considering this proposed RCT, there had been only one published study of the use of an IPC device beneath a below-knee plaster cast. This study was terminated due to the high rates of VTE in both the intervention and the control group (Domeij-Arverud et al., 2013). As the sample size was small, no conclusion can be drawn as to whether this is an unusually high occurrence of DVT in this particular patient group, or whether the IPC system may have had a causative effect. The fact that a number of patients complained of device failure in the first study using plaster casts leading to replacement of the IPC garments and the casts prior to the planned review dates in the study, suggested that the use of a hard and inflexible plaster cast may have been a contributory factor to the failure of the study.

I was reassured by my findings in Study Six that the haemodynamic effect of the IPC/JIT device was not impaired by its placement beneath a fibreglass cast, and that there might be less risk of device failure with prolonged use. Expecting the fibreglass cast to have more of flexibility or 'give' than a plaster cast, I hypothesised that the IPC/JIT device within a belowknee fibreglass cast might still be an option for a treatment arm in the proposed RCT. However, there were still a number of feasibility issues that needed to be addressed.

The Domeij-Averud study made no comment on the relative ease or difficulty of recruiting participants into their trial, and had selected a specific cohort: those who had undergone 
surgical Achilles tendon repair. My intended patient group was designed to reflect all potential participants that would be eligible for the proposed RCT, and the proportion of potential participants that would actually participate in a trial that involved the IPC/JIT needed to be established.

Once enrolled, participants would be required to utilise the IPC/JIT as much as possible while they were sitting or lying, including while sleeping. In a series of 621 recordings of foot pump use in 29 hip and knee arthroplasty patients, in only $37.2 \%$ of cases was the utilisation considered effective (Charalambous et al., 2003). Researchers found that with each postoperative day, there was a gradual, but significant reduction in correct utilisation of the foot pump device and that most utilisation occurred overnight, rather than when at rest during the day. The researchers postulated that this might be due to increased frequency of mobilisation each post-operative day, accompanied by an increased likelihood of patients forgetting or neglecting to re-start their foot pumps. In surgical wards, similar results have been found, with one audit finding that in only $48 \%$ of instances were IPC devices properly functioning (Anthony J. Comerota, Katz, \& White, 1992). In the situations where the devices were non-functioning, $84 \%$ involved sleeves not attached to patients and $16 \%$ of cases involved the pump units not operating. Therefore actual device utilisation over the study period would need to form one component of my feasibility study.

Skin integrity issues also needed to be taken into account. Dorsal foot abrasions and malleolar ulcers were reported in three (4.5\%) of 67 patients using plantar compression devices after total hip replacement (Santori, Vitullo, Stopponi, Santori, \& Ghera, 1994). There is one concerning case report of extensive circumferential bullae found on the calves and shins of an elderly patient who underwent calf IPC for three days after a hemiarthroplasty (Won, Lee, Suh, \& Koo, 2013). They were consequently diagnosed with fragile skin and a recommendation regarding regular skin integrity checks in this clinical setting was made. There has been a case report of skin maceration after application of a similar IPC foot-pump device placed within an emergency back-slabs applied pre-operatively to reduce traumatic swelling. Unfortunately in this instance the non-operational footpad was left on the patient, within the backslab after the patient was sent home with a 
conservative treatment plan instead of an operation. He returned eleven days later with grossly macerated skin on the lateral sole of his foot. In another study of foot compression for the reduction of swelling after ankle fracture, two of 15 patients withdrew, one due to pain, and one due to attribution of bruising on the dorsum of the foot to the IPC device (Thordarson, Ghalambor, \& Perlman, 1997).

To assess the feasibility of the IPC/JIT as a treatment arm in the proposed RCT in an outpatient setting, I went on to develop a feasibility study to address the specific issues of potential recruitment rates into the proposed RCT, likely participant adherence to the IPC/JIT device, possible adverse events associated with the use of the IPC/JIT device within a lower limb fibreglass cast between planned study visits. 


\section{STUDY EIGHT: A cohort feasibility study of an intermittent pneumatic compression device within a below-knee cast for the prevention of venous thromboembolism}

\section{Hypothesis}

The hypothesis for this study was that an IPC device within a below-knee fiberglass cast would be a feasible treatment arm in a proposed three-armed RCT of rivaroxaban $10 \mathrm{mg}$ daily, IPC and aspirin 100mg daily, and aspirin 100mg daily alone for the prevention of VTE in the clinical setting of lower limb immobilisation (proposed main RCT).

\section{Primary outcome variable}

The primary outcome measure was the proportion of eligible participants who participated in the feasibility study.

\section{Secondary outcome variables}

Secondary outcome variables included adherence to the proposed IPC/JIT in-cast regime, adverse events potentially associated with its use and proportion of potential patients who might not be eligible for recruitment to a trial arm that included the use of Rivaroxaban based on the following; already using an oral anticoagulant, being at very high risk of VTE, being treated for active upper gastro-intestinal ulcer disease, immobility $>2$ days prior to enrolment, significant liver disease, creatinine clearance $<30 \mathrm{ml} / \mathrm{min}$ ), or concomitant use of HIV protease inhibitors.

\section{Methods}

This was a prospective, open-label feasibility study of the IPC/JIT device placed within the lower limb cast of individuals aged 18 to 70 , who presented with a lower limb injury requiring a minimum of four weeks below-knee cast immobilisation. The exact ClopperPearson method was used to estimate $95 \%$ confidence interval proportions which would allow the development of an appropriate sample size for the proposed main RCT. 


\section{Participants and Methods}

Recruitment for the feasibility study occurred for six months, August 2015 to January 2016, with a further three months for follow-up of participants. Eligible participants were patients aged between 18 and 70 years presenting to Wellington Hospital Fracture Clinic with ruptured Achilles tendon, stable ankle fracture requiring a non-weight-bearing cast, or, if possible, ankle fracture with operative fixation and then cast immobilisation. Exclusion criteria included patients with a high risk for VTE, active gastric or duodenal ulcer, already using anticoagulation, immobility $>2$ days before enrolment, significant liver disease (including moderate to severe hepatic impairment especially when associated with coagulopathy), renal impairment (estimated creatinine clearance $<30 \mathrm{ml} / \mathrm{min}$ ), or concomitant use of HIV protease inhibitors.

Initially recruitment was limited to working hours, however by the middle of September it was identified that many potentially eligible participants presented on weekends, and so ethics approval was obtained at the end of September to extend recruitment over weekends, still within usual business hours of $8 \mathrm{am}$ to $5 \mathrm{pm}$. Investigators and orthopaedic nursing staff identified potentially eligible participants, informed them about the open-label trial, and asked them if they would like to participate. Those who declined to participate were given the opportunity to provide a reason for non-participation, and if the reason for not participating was because the treatment was a device rather than a medication, this was documented. A record was kept of potential participants, including those who were treated and discharged outside of working hours, and of all potential participants approached during the working day. This record was used to identify the proportion of eligible participants likely to be recruited into the proposed RCT.

Potential participants were approached as they arrived in the outpatient department and were given a participant information sheet by the department nursing staff. If they expressed interest in the study, I was called to explain the study and to answer any queries potential participants had. Participants were advised that they had the ability to withdraw from the study at any time. No study procedures were undertaken until after participants had signed written informed consent. 
Participants who agreed to be enrolled into the feasibility trial had the IPC with Jet Impulse Technology (IPC/JIT) placed under their lower limb cast (Figure 7.1) and the usual care of aspirin EC 100mg daily was also prescribed. They were reviewed fortnightly at the fracture clinic for up to eight weeks. In-cast pad changes were planned at weeks two and four and possibly six depending on adherence of participants to the device and the treatment plans of the orthopaedic consultant.

The IPC/JIT device was pre-set to inflate to $130 \mathrm{mmHg}$ once every minute, and participants had only to connect to the pump unit and turn the device on or off as required. To measure adherence with the device, participants were asked to use the IPC/JIT as much as possible while they were seated or immobile during the day and while in bed at night. Good adherence was defined as $60 \%$ of the total potential time available, namely 14.4 hours of a potential 24 hours per day. Total time, in hours, of IPC/JIT device usage was collected directly from the device at each clinic visit.

To assess ease of application of the device and any adverse events, nursing staff completed a semi-structured record regarding the practicalities of device placement and related issues such as skin condition of the lower limb after removal of the cast at each clinic visit. Adverse events and withdrawal reasons (if provided) were documented as they occurred. 
Figure 7.1: Placement of patient foot within JIT foot garment and fibreglass cast.
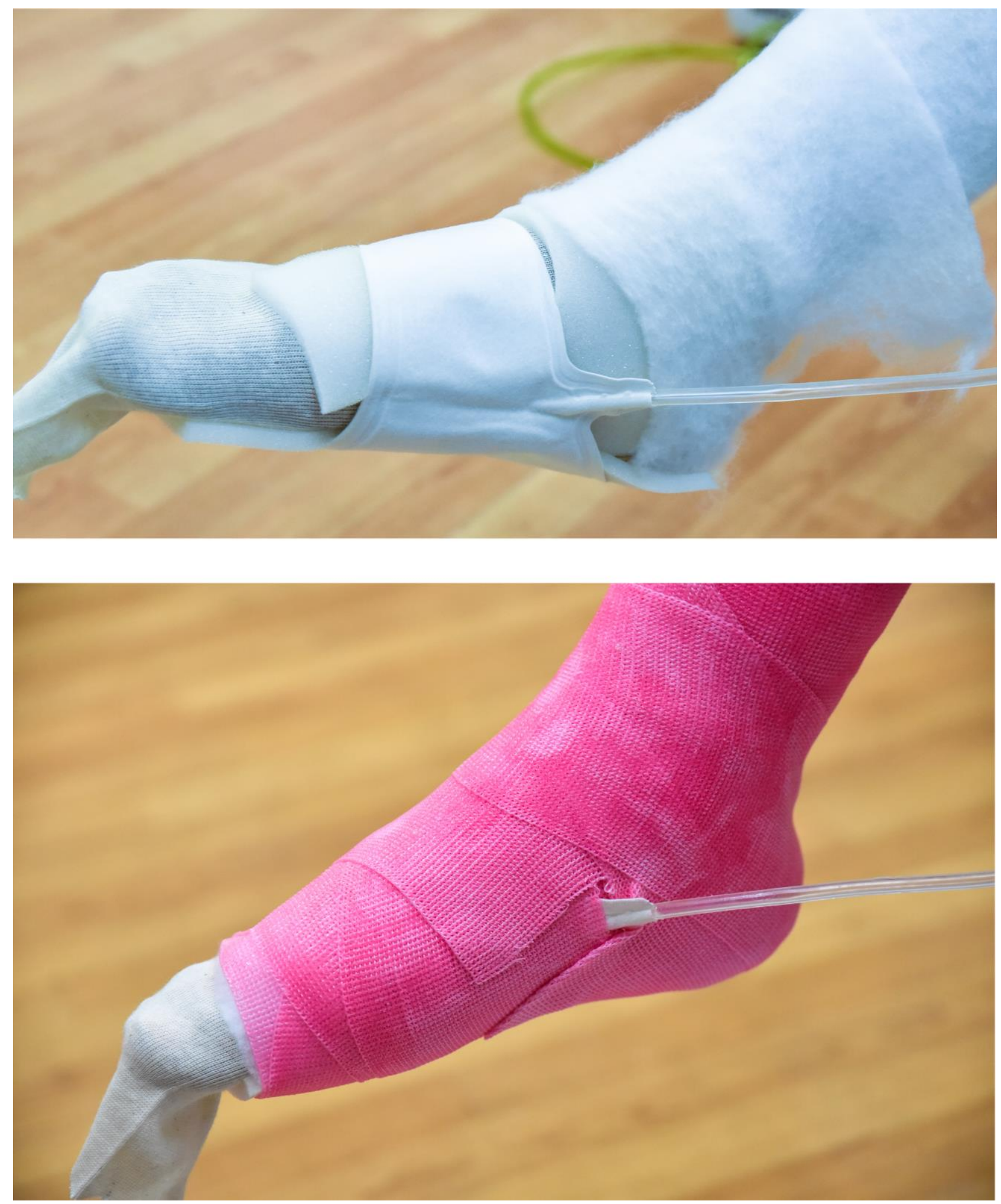
The VADOplex ${ }^{\circledR}$ VenaJet foot pump system (OPED, Oberlaindern, Germany) is an intermittent compression device that works with Jet Impulse Technology (JIT), to mimic the usual weight-bearing walking process. It comprises a $4.6 \mathrm{~kg}, 320 \mathrm{~mm}$ wide $\times 190 \mathrm{~mm}$ high $\mathrm{x}$ $200 \mathrm{~mm}$ deep (including mounting handle), bilateral pump unit, with an in-cast pad that is attached to the pump unit via an air tube (Figure 7.2). The pump mechanism mimics the natural full weight-bearing walking process, rapidly inflating a distal air-cell in the foot cuff to $130 \mathrm{mmHg}$, which then settles to $52 \mathrm{mmHg}$, followed by a proximal air-cell 0.3 seconds later, settling to $48 \mathrm{mmHg}$. After 6 seconds of compression at 48 to $52 \mathrm{mmHg}$, both air-cells deflate. This cycle is repeated every minute. The total hours the device has been in use, both with the garment in operation, and without the garment, is indicated on the device readout. These numbers were documented at each visit.

Figure 7.2: The VADOplex ${ }^{\circledR}$ VenaJet foot pump system.

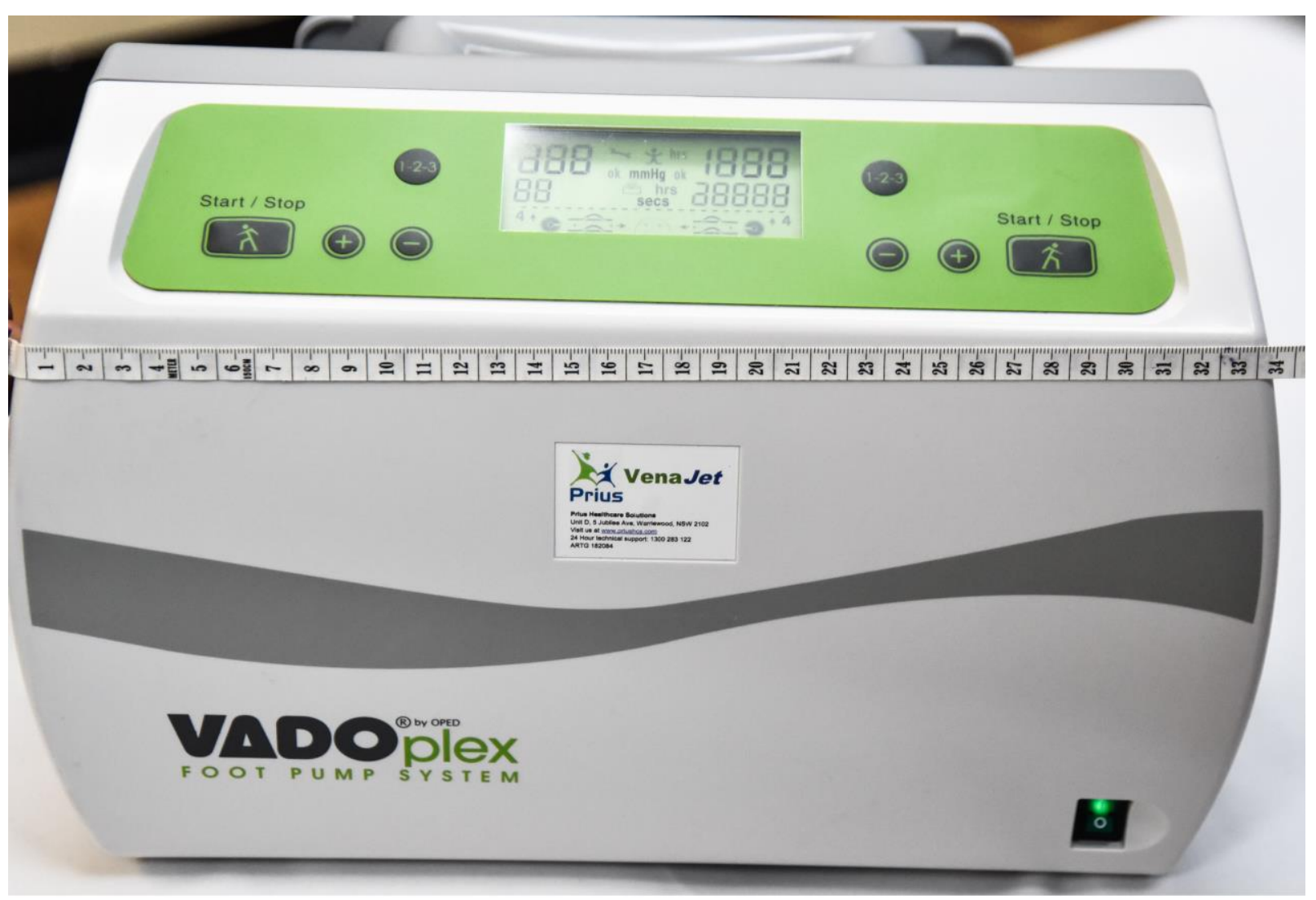


All participants were scheduled for ultrasound examination of the popliteal to femoral vein of the affected limb (accessible above the leg cast) prior to each clinic visit, and again for ultrasound of the entire limb after removal of the cast or moonboot at the end of their orthopaedic treatment period which was expected to be between six and eight weeks. At any time between clinic visits and during the ensuing six week follow up period, if participants experienced signs or symptoms of DVT or PE, they would have an ultrasound or CTPA respectively, and be treated according to Wellington Hospital protocols. All participants were educated about the symptoms of DVT and PE and advised to seek medical review should these occur. A final follow up phone call was done six weeks after completion of cast-immobilisation treatment to ensure there had been no VTE event after completing the study.

\section{Sample size and statistical methods}

A total of 70 participants in the feasibility trial provided over $95 \%$ power to rule out a recruitment rate of less than $50 \%$. It also provided $80 \%$ power with an alpha of $5 \%$ to rule out a complication rate of at most $33 \%$ if the anticipated complication rate was $10 \%$; and to rule out adherence of less than $60 \%$ if the adherence rate was $75 \%$. The main analyses planned were to estimate $95 \%$ confidence interval proportions by the exact ClopperPearson method and to compare the achieved proportions with those outlined in the sample size calculation.

SAS version 9.4 was used. 


\section{Results}

Figure 7.3: Flow of eligible participants for study

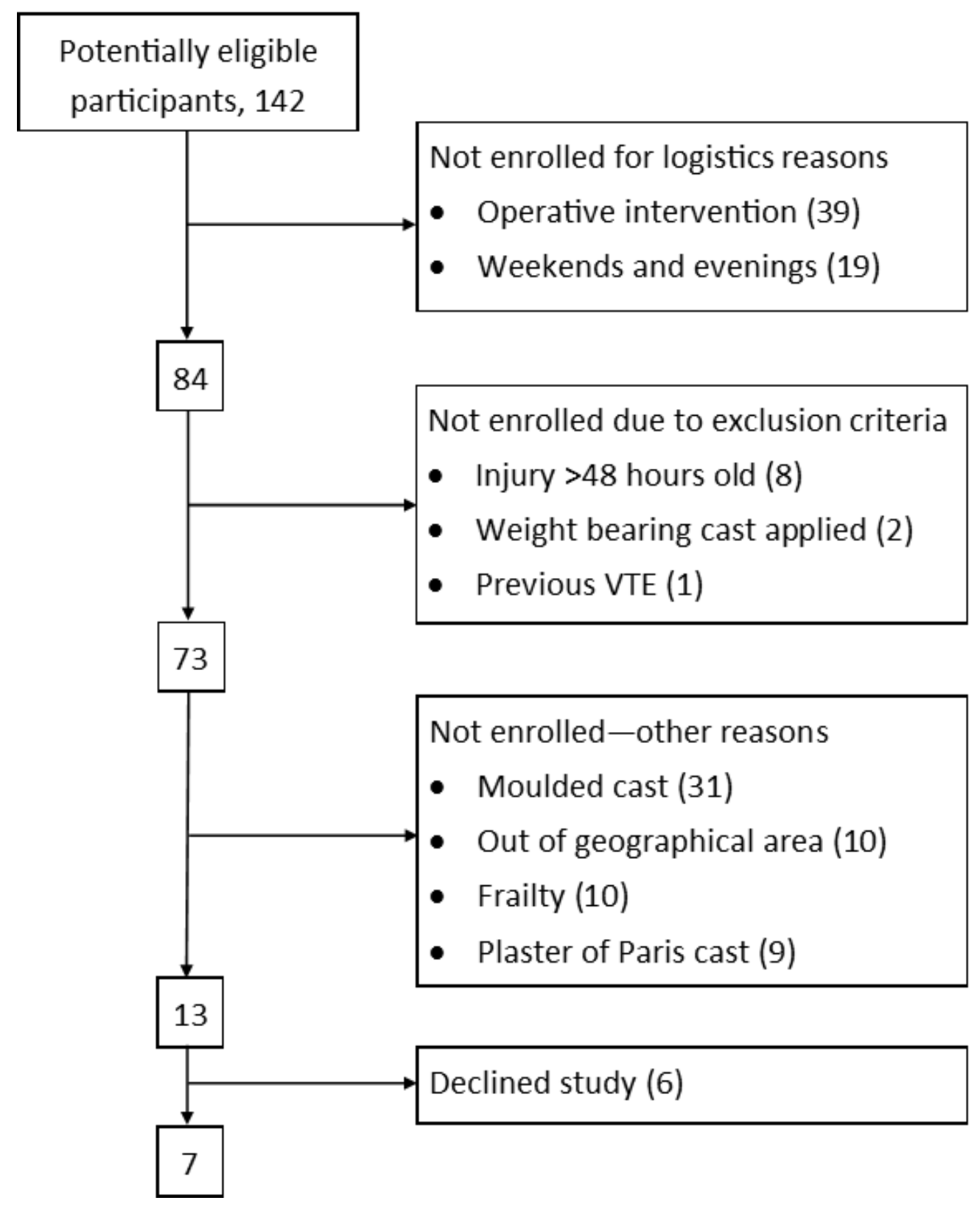


Only $7 / 142$ (5\%) of potentially eligible participants were enrolled (Table 7.1), with an exact $95 \% \mathrm{Cl}$ of $2 \%$ to $9.9 \%$, P-value for comparison with a rate of $50 \%<0.001$. There were 58 (41\%) potentially eligible participants who were not enrolled for logistics reasons; $39(27 \%)$ were admitted for operative intervention, and due to theatre logistics, could not be enrolled and 19 (13\%) presented on weekends and evenings before the change in study protocol. There were 77 (54\%) potentially eligible participants who were not enrolled due to reasons that were not anticipated at the time of design of the feasibility study, such as a moulded cast preventing the placement of the IPC/JIT, living out of geographical area thus making follow-up of participants impracticable, frailty of potential participants and use of a Plaster of Paris cast, the integrity of which might be impaired by the IPC/JIT device.

Six potential participants declined due to the perceived impracticality of using the IPC/JIT device. There were no documented issues with skin integrity at initial device and cast placement.

There were no issues with initial device placement by fracture clinic staff. A description of all 142 potential participants is shown in Table 7.2. 
Table 7.1. Flow of potential participants through the feasibility study

\begin{tabular}{cc}
\hline Recruitment status (N=142) & N (\%) \\
\hline Not enrolled - Logistic Reasons & $58(40.8)$ \\
$-\quad$ Operative intervention & $39(27.5)$ \\
$-\quad$ Weekend and evenings & $19(13.4)$ \\
\hline Not enrolled - Other reasons & $77(54.2)$ \\
$-\quad$ Moulded cast & $31(21.8)$ \\
$-\quad$ Out of geographical follow up area & $10(7.0)$ \\
$-\quad$ Frailty & $10(7.0)$ \\
$-\quad$ Plaster of Paris cast & $9(6.3)$ \\
\hline Not enrolled - Exclusion criteria & $11(8.2)$ \\
$-\quad$ Injury $>48$ hours prior & $8(5.8)$ \\
$-\quad$ Other VTE risk factor & $2(1.4)$ \\
\hline Declined study & $1(1)$ \\
\hline Enrolled in study & $6(4.2)$ \\
\hline
\end{tabular}


Table 7.2. Description of potential and enrolled participants in feasibility study

\begin{tabular}{|c|c|c|}
\hline & Not recruited ( $\mathrm{N}=135)$ & Recruited (N=7) \\
\hline Continuous variables & \multicolumn{2}{|c|}{ Mean (SD) } \\
\hline Age (years) & $43.1(15.0)$ & 39.4 (7.9) \\
\hline Categorical variable & \multicolumn{2}{|c|}{$\mathbf{N}(\%)$} \\
\hline Female & $75(56)$ & $3(43)$ \\
\hline \multicolumn{3}{|l|}{ Ethnicity } \\
\hline - European & $87(64.4)$ & $2(28.6)$ \\
\hline - Maori & $19(14.1)$ & $342.9)$ \\
\hline - Pacific Island & $11(8.1)$ & $2(28.6)$ \\
\hline - Asian & $10(7.4)$ & $0(0)$ \\
\hline - Other & $8(5.9)$ & $0(0)$ \\
\hline \multicolumn{3}{|l|}{ Day presenting } \\
\hline - Monday & $23(17.0)$ & $2(28.6)$ \\
\hline - Tuesday & $19(14.1)$ & $1(14.3)$ \\
\hline - Wednesday & $19(14.1)$ & $1(14.3)$ \\
\hline - Thursday & $10(7.4)$ & $1(14.3)$ \\
\hline - $\quad$ Friday & $22(16.3)$ & $1(14.3)$ \\
\hline - Saturday & $27(20.0)$ & $0(0)$ \\
\hline - Sunday & $15(11.1)$ & $1(14.3)$ \\
\hline \multicolumn{3}{|l|}{ Injury } \\
\hline - $\quad$ Ankle Fracture & $64(47.4)$ & $1(15)$ \\
\hline - $\quad$ Achilles tendon & $42(31.1)$ & $6(85)$ \\
\hline - Fibula Fracture & $13(10.4)$ & - \\
\hline - Other injury & $16(11.1)$ & - \\
\hline
\end{tabular}




\section{Participants enrolled to use the IPC/JIT device}

Of the seven participants enrolled in the feasibility study three participants did not complete the minimum four week period of IPC/JIT usage; one changed to a weight-bearing cast after one week and the device was removed, one withdrew after developing an ulcer on the dorsum of their foot in the initial two week period (Figure 7.4), and one withdrew after two weeks due to maceration of the skin. Four participants completed four weeks of IPC/JIT device usage. One participant that completed the four week intervention had a DVT diagnosed at scheduled four week ultrasound, and was treated with rivaroxaban. No participants developed symptomatic VTE in the six week follow up period. Excluding the participant who changed to a weight-bearing cast the complication rate was $3 / 6$ (50\%) with $95 \% \mathrm{Cl}$ of 11.8 to 88.2 ; P-value for a comparison with $33 \%$ of 0.63 .

Figure 7.4: Dorsal foot ulcer with IPC / JIT foot garment utilisation.

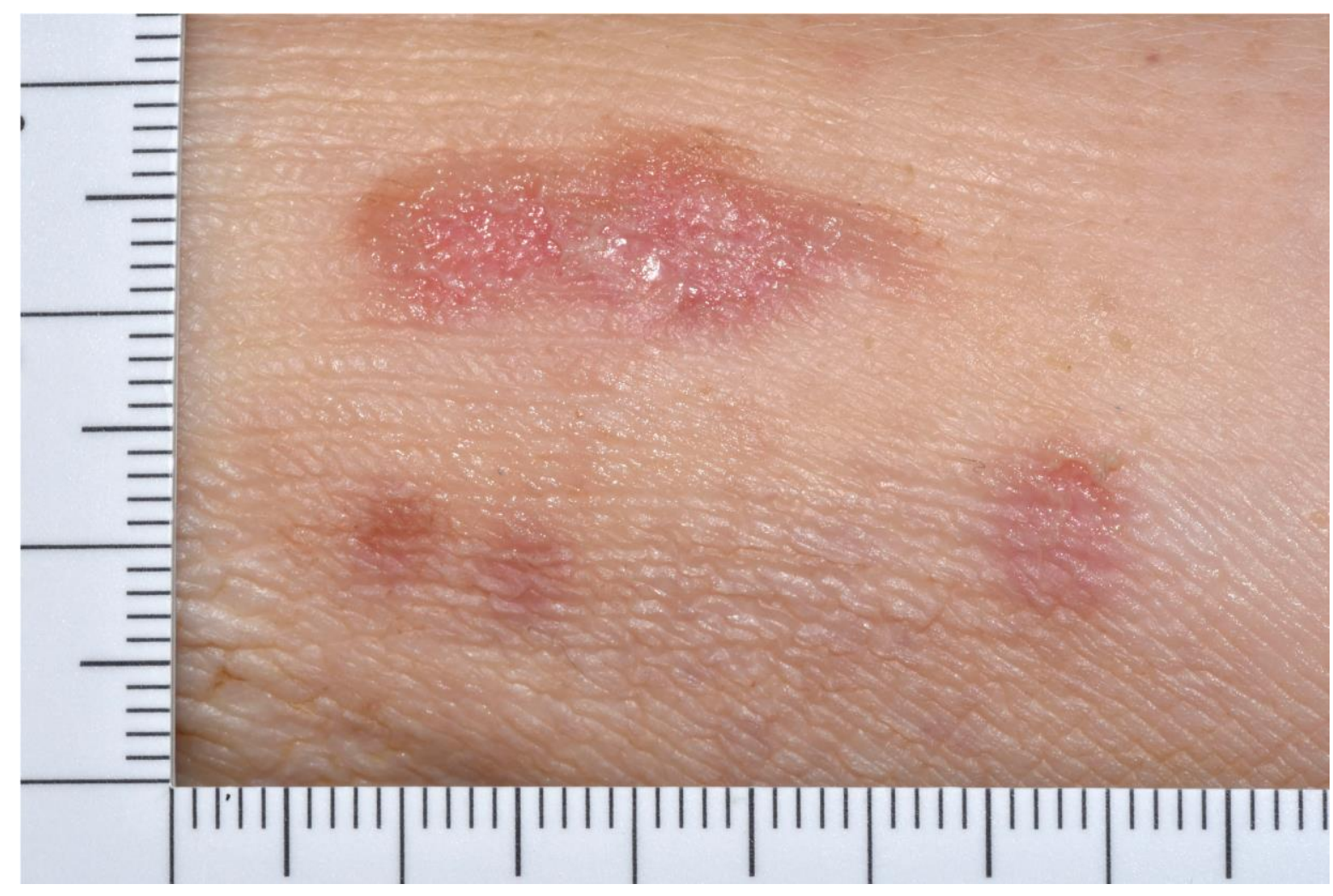




\section{Participant adherence to IPC/JIT and feedback about the device}

The seven participants were issued the IPC/JIT devices and used the device for a total of 148 days. For these participants a total of 608 hours of use were recorded. The mean (range) of daily use was 4.1 hours (1.9 to 10.2). No participant met the proposed adherence target of an average of 14.4 hours per day in any of the two-week periods with a $95 \%$ confidence interval for adherence of 0 to $41 \%$, P-value for comparison with $60 \%, 0.003$. Of the seven participants, six did not use the device at night as it interfered with their sleep. All participants found the pump unit difficult to move around while they were non weightbearing and on crutches, and tended to leave the pump unit in a single location where they were likely to spend most of their time. One participant attempted to take the pump unit to and from work, but found even with a backpack, that the pump unit was too large and awkward to carry around while on crutches. All participants indicated that they would use an IPC/JIT system again, but that the pump unit would have to be much smaller and lighter to allow ease of use.

\section{Exclusion criteria for rivaroxaban arm of proposed RCT}

Of the 142 potentially eligible participants identified in the study, $5(3.5 \%)$ were already prescribed an anticoagulant, $3(2.1 \%)$ had a high risk of VTE (two had active nephritis and one had a history of VTE) and $1(0.7 \%)$ was on treatment for an active gastric ulcer. In all 9/142 (6.3\%) would not have been eligible for Rivaroxaban treatment, $95 \% \mathrm{Cl} 2.9$ to 11.7. None of the remaining 133 (91.7\%) participants had any of the remaining exclusion criteria: immobility $>2$ days prior to enrolment, significant liver disease, creatinine clearance $<30 \mathrm{ml} / \mathrm{min}$ ) or concomitant use of HIV protease inhibitors. 


\section{Conclusion}

This feasibility study shows that the IPC device utilising JIT technology is not a feasible randomisation arm in a RCT assessing VTE prevention rates in lower limb immobilisation. Furthermore, it is clearly not a therapeutic option for VTE prophylaxis in this clinical setting, regardless of its potential efficacy.

While this study was of the VADOplex ${ }^{\circledR}$ VenaJet foot pump system IPC/JIT device specifically, the findings in this study are broadly generalisable to individuals utilising IPC foot garments within rigid lower limb casts for prolonged periods in the outpatient community. The size and weight of the pump unit limiting patient mobility, interference with sleep leading to lack of use overnight, and skin related adverse events are likely to occur with other IPC systems in this clinical setting, thus impacting ongoing patient adherence and denying maximal antithrombotic effect.

It is interesting to compare outpatient utilisation of the IPC/JIT device when compared to studies of foot IPC use within hospital inpatient settings. In a study of patients after a total joint arthroplasty Pitto and Young reported that while 5\% of patients discontinued IPC use between two and six days after initiation of use, foot pump devices had been used by the remaining $95 \%$ of patients for 15.9 hours daily (Pitto \& Young, 2008). Charalambous and colleagues assessed adherence to foot-pump usage in an inpatient setting after joint arthroplasty, and found that as patients got in and out of bed more frequently, their foot pumps were re-applied less often, and that only $60 \%$ of patients used foot pumps at night when confined to bed (Charalambous et al., 2003). In this study, the IPC/JIT device was used for four hours/day and six of seven participants did not use the device at night. It is likely that in an unsupervised environment participants may be less likely to utilise the device, particularly overnight compared to an inpatient group. Adherence might be improved by utilising a smaller, more portable pump unit that was easier for participants to move around with. Providing more than one of the current devices to each participant to allow placement at strategic locations at home or at work, while possible, is likely to prove too expensive.

It is informative to compare the complications associated with IPC/JIT use in this study with those experienced in other trials. Two out of seven (28.5\%) participants suffered 
complications associated with the IPC/ JIT device; one a dorsal foot ulcer and one who suffered skin maceration. This is higher than skin-related adverse events reported in previous studies where dorsal ulcers, skin blisters and malleolar sores have been reported in $0 \%$ of 42 patients after hip surgery (Asano, Matsubara, Suzuki, Morita, \& Shinomiya, 2001), $6.7 \%$ of patients receiving plantar venous compression after total hip arthroplasty (Bradley et al., 1993), 4.5\% in patients using a plantar compression device after total hip replacement (Santori et al., 1994) and 7.5\% of patients using pre-operative plantar compression after ankle fracture (Caschman, Blagg, \& Bishay, 2004). Skin maceration is not uncommon within fibreglass casts, due either to excess sweating or inadvertent wetting of the cast, thus we were uncertain that the IPC/JIT device was the direct cause of the maceration. After this event, we started placing more protective material between the footpad and the skin and no further episodes occurred.

Foot pump devices have been shown to increase lower limb venous flow (Charles et al., 2013; Delis et al., 2000; Morris, Woodcock, Pitto, \& Young, 2004), and a number of early studies have shown that foot pump prophylaxis with or without graduated compression stockings and/or pharmacological prophylaxis significantly reduces the risk of VTE after major orthopaedic surgery compared to; compression stockings alone (Fordyce \& Ling, 1992), or compression stockings and heparin (Bradley et al., 1993; Santori et al., 1994). These findings, in conjunction with the high rates of VTE found in the clinical setting of lower limb immobilisation (Irene Braithwaite et al., 2016; B. Healy, Beasley, et al., 2010; Mark Testroote et al., 2014), raise the question of potential IPC/JIT prophylaxis in this situation, which would eliminate the risk of bleeding that exists with pharmacological prophylaxis.

Since the inception of this feasibility study, Domeij-Arverud and colleagues (Domeij-Arverud et al., 2013) have published findings related to the use of an IPC and plastic foot cuff under a Plaster of Paris cast after Achilles tendon repair. They found that nine of 14 participants randomised to IPC treatment and six of 12 who received no intervention developed DVT during the intervention period. IPC malfunction and the need to replace the cast correlated with a higher risk of VTE at 2 weeks resulting in the study being halted, suggesting that IPC prophylaxis in this clinical setting is no better than, and in fact is possibly worse than no 
prophylaxis. In this study, I enrolled only patients with fibreglass casts, there were no IPC/JIT malfunctions and no unscheduled cast changes were necessary. One in seven participants in this study was found to have a DVT on radiological screening, however given the small number of participants that utilised the IPC/JIT in this study, I cannot draw a conclusion about the likely VTE rates with this intervention.

If the randomised treatment was dropped from the proposed $\mathrm{RCT}$, and all the limiting logistics factors of the non-recruited group were suitably addressed e.g. full out of hours recruitment; access to the operating theatre post-operatively for device placement, about 130 patients would be eligible in each six month period. In this modified RCT, 200 participants would be required in each treatment arm (a total of 400 ) to ensure $90 \%$ power with an alpha of $5 \%$ to detect a $50 \%$ relative reduction in VTE events with rivaroxaban compared to the control arm (aspirin), assuming a 20\% drop out rate. This suggests that the single Wellington Hospital site would have the potential to fully recruit the proposed RCT within a two year period, even if the recruitment rate was $80 \%$.

In conclusion, the IPC/JIT device placed within a lower limb fibreglass cast is not a feasible randomisation arm for the proposed RCT investigating the rates of VTE in the clinical setting of outpatient temporary rigid lower limb immobilisation due to low recruitment rates, poor adherence to treatment regimens and high rates of adverse events. The characteristics of potential participants suggest that recruitment into the rivaroxaban and aspirin arms of the proposed RCT from a single location is achievable. 


\section{Chapter 8: Conclusions}

In this thesis I have reported the association between work- and computer-related immobility and VTE, and extended knowledge in this area by reporting the association between sedentary occupations and VTE. I then investigated a possible intervention, the Legflow device, to reduce venous stasis in seated workers by firstly measuring the haemodynamic efficacy of the Legflow and then assessing its feasibility in the sedentary workplace.

To understand what is already known about VTE risk associated with prolonged work- and computer-related seated immobility, I completed a review of the medical literature assessing this possible risk factor. While there are a number of trials in the medical literature that have assessed the risk of VTE associated with air travel and other travel modalities, the majority of the literature addressing work- or computer-related immobility and VTE is limited mainly to case reports. The two case-control studies assessing risk in this context have shown that the risk of VTE increases with increasing mean hours seated per day and with increasing periods of time sitting without actually getting up (B. Healy, Levin, et al., 2010; West et al., 2008).

I confirmed this increased risk by completing a case-control study of 200 VTE patients (cases) and 200 patients with upper limb injuries (controls), where I found that for the mean number of hours seated in a 24 hour period, the risk of VTE increases by $8 \%$ for every additional hour seated. I also found that for the maximum number of hours seated in a 24 hour period, the risk of VTE increases by $4 \%$ for every additional hour seated. In the course of this thesis, it has become apparent to me that future studies should contain continuous primary outcome variables rather than dichotomous ones, and that they should investigate sitting in all aspects of life; at home, at work and socially.

In order to assess the possible association between VTE and specific occupations, only previously reported in commercial airline pilots (Kuipers et al., 2014), I combined and analysed the data from my own case-control study and that of Healy and colleagues (B. Healy, Levin, et al., 2010). I found that there was a $79 \%$ increased risk of VTE in sedentary 
occupations. I was unable to demonstrate an increased risk of VTE within the specific occupational classes of administrative and clerical workers, or ICT workers, however the point estimates were consistent with increased risk. Future larger studies may be able to demonstrate an increased risk of VTE in these specific occupational classes.

These findings are important given that in excess of $40 \%$ of the workforce is likely to be in low activity occupations (Brownson et al., 2005) and those that work in low activity occupations are unlikely to compensate for the lack of activity by increasing activity in their leisure time (Jans et al., 2007). From a broader public health perspective, these findings are also consistent with a number of studies that demonstrate adverse health outcomes and increased mortality rates in sedentary workers (Eriksen et al., 2015; G. N. Healy et al., 2008; Mummery et al., 2005)

While there have been a number of studies reporting interventions to reduce sedentariness in the workforce, they have generally been resource intensive and with only transient effects (Dugdill et al., 2008). No successful intervention for the prevention of DVT in the workplace has been reported in the medical literature. The Legflow has been designed with the intention of reducing VTE risk in the sedentary workforce and during prolonged travel, however, for it to be a feasible intervention its haemodynamic effects had to be established and its feasibility in the sedentary work environment had to be demonstrated.

I showed in a haemodynamic study of 10 healthy volunteers that use of the Legflow while seated resulted in a 12-fold increase in peak systolic venous velocity compared to an immobile leg after 20 minutes of sitting in an office chair. Thus the Legflow might be a useful tool in reducing venous stasis that occurs while sitting.

I went on to test the feasibility of the Legflow in its intended clinical setting: the sedentary working environment. In a study of 96 office workers who were instructed to use the Legflow hourly while at work, just over half used used the Legflow for 4 or more times per day. Two thirds of participants used the Legflow every day they were at work. Just over half of all participants indicated they would be likely or very likely to use the Legflow at work and / or on a plane. Also of public health and employee health and safety significance, I have 
shown that three quarters of this workforce have one or more risk factors for VTE, and that in a sedentary workforce up to $5 \%$ of workers can have up to four risk factors for VTE. Reducing venous stasis associated with seated immobility could reduce the number of employees at risk of VTE on the sedentary working environment.

This is the first series of studies that have shown the haemodynamic effectiveness of a device designed to reduce venous stasis in seated workers, and proven its feasibility in the sedentary working environment.

I also assessed the risk of VTE associated with lower limb immobilisation in rigid below-knee casts by establishing the odds for association of lower limb immobilisation with VTE, and the proportion of VTE patients seen at Wellington Hospital VTE clinic with this potentially preventable risk factor. In a new non-pharmacological approach I assessed the feasibility of an IPC device for the prevention of VTE in this clinical setting by first measuring the haemodynamic effectiveness of this device within a below-knee fibreglass cast, and then assessing patient use and adherence of such a device in the outpatient orthopaedic setting.

I reviewed the medical literature addressing VTE risk in the clinical setting of lower limb immobilisation and found a range of papers addressing diverse patient groups, reporting a wide variation in the incidence of VTE, disagreement as to the clinical significance of the asymptomatic DVTs found in studies that used screening protocols, and despite RCTs and an associated Cochrane review that demonstrated the benefit of LMWH in reducing VTE in this patient group, no clear guidelines for prophylaxis of VTE in this clinical setting.

Utilising the same combined case-control data with which I assessed VTE risk by occupational groups, I found that lower limb immobilisation represented a 73-fold increased risk of VTE, and that this patient group represented the most common potentially preventable case of VTE cases presenting to VTE outpatient clinics at Wellington Hospital, with one in seven patients having undergone lower limb immobilisation within four weeks of their presentation with VTE.

Preventive measures have been investigated in lower limb immobilisation but the majority have been focused on pharmacological intervention for which adverse bleeding events may 
present an issue. LMWH, while proven in this clinical setting is not approved or funded for this use in New Zealand and rivaroxaban has not been proven in this clinical setting.

While aspirin reduces the risk of VTE in orthopaedic patients (Antiplatelet Trialists' Collaboration 1994; Pulmonary Embolism Prevention (PEP) Trial Collaborative Group 2000), I showed in my audit of 200 patients with Achilles tendon injuries who have been immobilised in a lower limb cast that aspirin 100mg daily appears to have had little impact on VTE rates compared to no intervention at all. Since the introduction of $100 \mathrm{mg}$ aspirin daily for routine prophylaxis of VTE in lower limb immobilisation, the incidence of symptomatic, radiologically confirmed VTE in 200 patients was $6.4 \%$. This was similar to the incidence of VTE (6.3\%) identified in a previous audit when no prophylaxis was routinely prescribed.

Foot-pump IPC is a possible non-pharmacological intervention for the prevention of VTE in lower limb immobilisation that does not confer an increased risk of bleeding. However, for an IPC device to be a feasible intervention I needed to demonstrate its haemodynamic efficacy while operating within the constraints of a below-knee cast, and to established its feasibility within the clinical setting for which it was intended: outpatients undergoing lower limb immobilisation.

In my haemodynamic study I demonstrated that the Vena Jet pump (VADOplex) and its' associated under-cast foot garment (marketed as IPC with Jet Impulse Technology (IPC/JIT) in New Zealand) was effective in increasing popliteal vein peak systolic velocity within a below-knee fibreglass cast. Therefore the IPC/JIT presented itself as a potential nonpharmacological option for the prevention of VTE in the clinical setting of lower limb immobilisation.

I then showed in a feasibility study that the IPC/JIT was not going to be a feasible option either as a treatment arm in a possible RCT, or as a treatment modality in the clinical setting of lower limb immobilisation. Its' feasibility was affected by a number of factors: logistics issues and the use of moulded casts prevented a widespread implementation IPC / JIT in the target patient group, there was poor adherence to the proposed treatment regime by those 
individuals that did agree to use the device, and there was a high rate of adverse events in the small number of participants recruited. However, an assessment of the characteristics of all potential participants in the feasibility study suggests that this patient group might be a suitable recruitment pool for a two arm RCT of rivaroxaban $10 \mathrm{mg}$ daily versus aspirin $100 \mathrm{mg}$ daily in the prevention of VTE in lower limb immobilisation.

In conclusion, increasing seated immobility for work- and computer-use is associated with VTE, with an increased risk for each additional hour seated. Sedentary professions have a $70 \%$ increased risk of VTE compared with non-sedentary occupations. The Legflow increases venous blood flow in seated adults and is a feasible device for use in the sedentary working environment. Lower limb immobilisation confers a 73-fold increased risk of VTE and represents the most common preventable risk factor reported by patients in the four weeks prior to their VTE event. The introduction of aspirin 100mg daily for routine VTE prophylaxis during lower limb immobilisation appears to have had little impact on VTE rates compared to when no prophylaxis was routinely prescribed. The haemodynamic effect of the IPC/JIT is not impaired by placement within a below-knee fibreglass cast, but it is not a feasible option as a treatment arm in a RCT, nor as a treatment modality for the prevention of VTE in the clinical setting of lower limb immobilisation. 
Appendix I. Venous thromboembolism risk associated with protracted work- and computer-related seated immobility: A case-control study. Participant Information Sheet 


\section{THE EFFECT OF WORK AND RECREATIONAL ACTIVITIES ON THE RISK OF DEVELOPING OF BLOOD CLOTS}

STUDY CONTACT:

Dr Irene Braithwaite Ph: 048050147

\section{INTRODUCTION}

You are invited to take part in a clinical research study. Please take the time to read this information sheet carefully to determine if the study is of interest to you. You may wish to discuss the information in this sheet with your family or whanau. Please ask us if you have any questions about the study. Your involvement in this study is voluntary and you have the right not to take part and to withdraw at any time.

\section{ABOUT THE STUDY}

\section{What is the basis of the study?}

Venous thromboembolism is a condition in which you develop blood clots in your veins. The blood clots usually develop in your legs (deep vein thrombosis) but occasionally can break away and spread in your blood stream to your lungs (pulmonary embolism). One of the main risk factors for blood clots is immobility (sitting still) which results in pooling of blood in the veins. The immobility associated with air travel has been shown to be associated with the risk of developing blood clots. However there are few studies looking at the risk of being seated for long periods of time for other activities. This study is investigating whether work habits and certain recreational activities that result in prolonged periods of sitting may also increase the risk of developing a blood clot.

\section{What are the aims of the study?}

To determine whether sitting still for long periods at work or during recreational activities is a risk factor for developing blood clots.

\section{How many participants will be involved?}

There will be 400 participants in total, aged between $18-65$ years. This will include 200 participants who have experienced a blood clot in a vein or in the lungs, within the previous 6 months (the 'case' 
group). There will also be 200 participants who have presented to the Fracture Clinic with an upper limb injury due to trauma, such as a laceration or a fracture (the 'control' group).

\section{Where will the study be held?}

The study will be conducted at one of the following sites:

- The respiratory outpatients department at Wellington Hospital, within the Clinical Measurements Unit

- The haematology outpatients department at Wellington Hospital, within the Blood and Cancer Centre

- $\quad$ The Kenepuru general outpatients department at Kenepuru Hospital

- $\quad$ The Fracture Clinic at Wellington Hospital

\section{What will I have to do?}

Your possible risk factors for blood clots will be assessed by a questionnaire. You will be asked to fill this out with the help of one of the investigators. It will ask you questions about your family history, recent travel, medication, other medical conditions and any strenuous exercise undertaken prior to developing your blood clot. It will also ask you about any time spent sitting in front of the computer, and if you are in paid employment it will ask you about the time you spent sitting whilst at work. The questionnaire will take approximately 10 minutes to complete.

\section{Do I have to take part in this study?}

Your participation is entirely voluntary (your choice). You do not have to take part in this study, and if you choose not to take part it will in no way affect your future health care or treatment.

If you do agree to take part, you do not have to answer all the questions, and you may stop the interview at any time. You are free to withdraw from the study at any time, without having to give a reason.

\section{Will I be able to have somebody with me?}

Yes, you are welcome to have a family or whanau member or friend with you if you wish.

Will I be able to have an interpreter? 
Yes, interpreters are available on request.

\section{Will the answers I give on the questionnaire be kept confidential?}

If you decide to participate in the study, the study doctor and MRINZ staff will collect medical and personal information about you as part of doing the study.

By agreeing to take part in this research, you will allow your medical information and results to be seen by people such as auditors and regulatory authorities (for example the Central Regional Ethics Committee) who check that the research was done properly. This process will in no way violate the confidentiality of the participants.

No material which could personally identify you will be used in any reports on this study. Your personal information (for example your gender, age and medical conditions) and other information will be identified by a number (i.e. coded).

No material which could personally identify you will be used in any reports on this study. For the duration of the study, any documentation will be kept in a locked office, and on completion of the study it will be stored securely for 16 years to comply with Good Clinical Practice guidelines and then destroyed.

\section{Will I be able to find out the results of the study?}

Yes, the results of the study will be available on completion of the study. You have the option of requesting a copy of the results to be sent to you, which you can select upon signing the consent form.

Will taking part cost me anything?

No, it will not cost you anything to participate.

\section{Where can I get more information about the study?}

You can call the researcher whose details are on the front page of this information sheet. An interpreter can be provided.

If you have any concerns about the study, feel free to call the Central Regional Ethics Committee, Telephone (04) 4962405. Or you can contact the Health Advocates Trust on 0800423638 (0800 4ADNET) 


\section{STATEMENT OF APPROVAL}

This study has received ethical approval from the Central Regional Ethics Committee.

\section{Compensation:}

In the unlikely event of a physical injury as a result of your participation in this study, you may be covered by ACC under the Injury Prevention, Rehabilitation and Compensation Act. ACC cover is not automatic and your case will need to be assessed by ACC according to the provisions of the 2002 Injury Prevention, Rehabilitation and Compensation Act. If your claim is accepted by ACC, you still might not get any compensation. This depends on a number of factors such as whether you are an earner or non-earner. ACC usually provides only partial reimbursement of costs and expenses and there may be no lump sum compensation payable. There is no cover for mental injury unless it is a result of physical injury. If you have ACC cover, generally this will affect your right to sue the investigators.

If you have any questions about ACC, contact your nearest ACC office or the investigator.

\section{Contact}

If you have any questions about the study you can contact:

Dr Irene Braithwaite - Telephone: 04-805 0147 
Appendix II. Venous thromboembolism risk associated with protracted work- and computer-related seated immobility: A case-control study. Questionnaire: Cases 


\section{SIT 3 questionnaire - Cases}

Date:

Name:

Hospital Number:

Ethnicity:

Date of Birth:

Gender:

(

Weight:

Height:

Date of presentation:

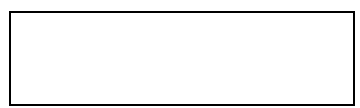

Admitted to hospital?

YES/ NO

Date of onset of symptoms of VTE:

Occupation (at date of onset of symptoms):

ANZCO Occupation code (11-94)

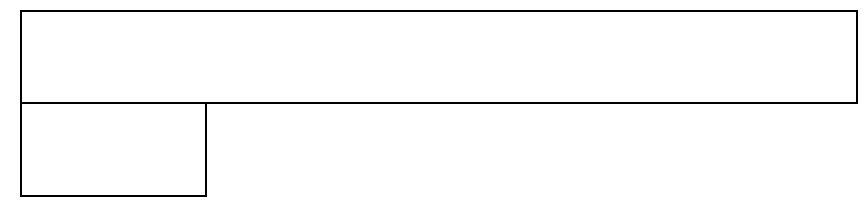

Previous history of VTE - specify:

1.

2. 
Family history of VTE - specify:

Parents:

Siblings:

Other:

\section{Section II: VTE risk factors}

Part A: In the 4 weeks prior to onset of symptoms that led to presentation:

Long distance travel ( $\geq 4 \mathrm{hrs})$ :

Car
Time before onset of symptoms (days)

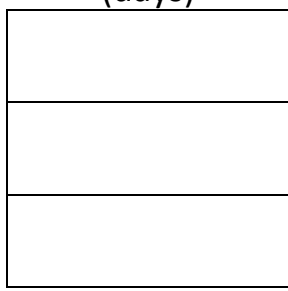

Duration of travel Max. hours Total per sector duration (hrs)
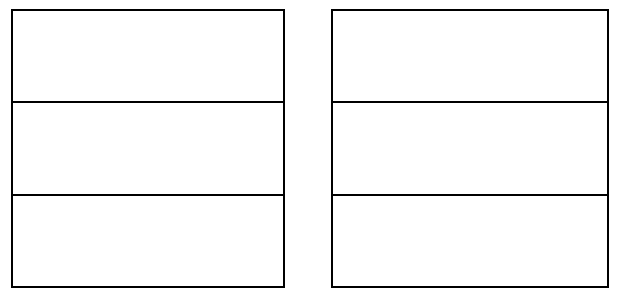

Part B: In the 4 weeks prior to onset of symptoms that led to presentation:

Other risk factors:

Surgery

Other immobility (e.g. Hospital admission, drug overdose, , wheelchair bound, bedrest) $\geq 2$ days Cast/plaster and/or traumatic injury

Pregnancy or hormonal treatment

Malignancy or chronic disease

Smoking

History of varicose veins
Yes No Details

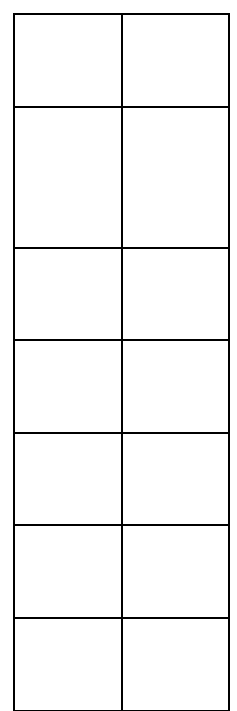


Part C: In the 4 weeks prior to onset of symptoms that led to presentation:

\section{Strenuous exercise ( $\geq 30$ mins):}

Details of the form of exercise

Cycling

Rowing /kayaking / waka ama

Weights (i.e. squats, lunges)

Long distance running

Other strenuous exercise

Note: "strenuous exercise" is defined as: cardiovascular or weight related exercise that involves repetitive physical

Specify whether road or MTB, gym equipment etc

Frequency and duration of exercise No of Longest duration (hrs)

times:
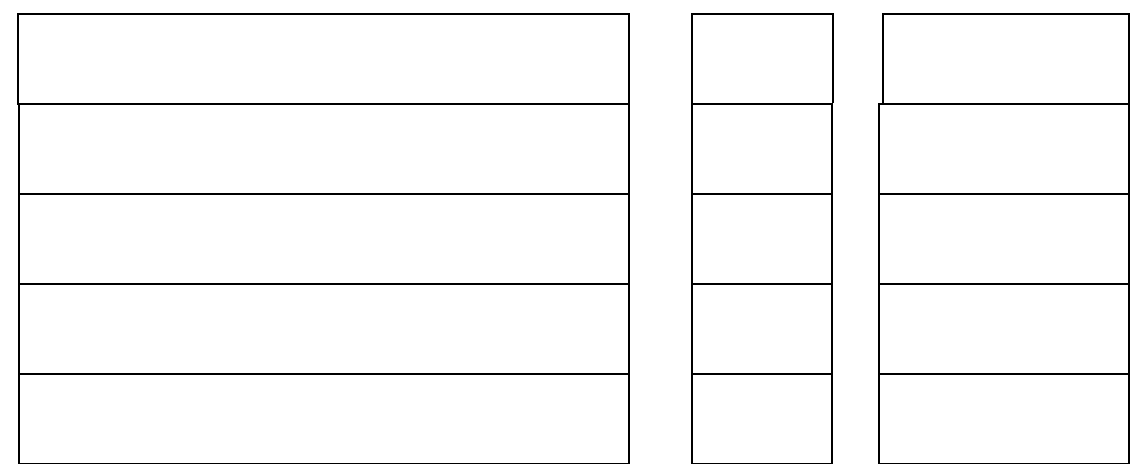
movements 


\section{Section III: Medications}

In the 4 weeks prior to onset of symptoms that led to presentation:

Anti-thrombotic agents

Yes No Details:

Enoxaparin

Warfarin

Aspirin

Clopidogrel

Dipyridamole

Other

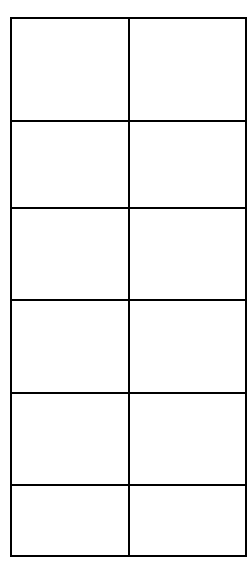

Pro-thrombotic agents

Anti-cancer medications

HRT

OCP

Tranexamic acid

Other

\begin{tabular}{|l|l|}
\multicolumn{1}{c|}{ Yes } & \multicolumn{1}{c}{ No } \\
\hline & \\
\hline & \\
\hline & \\
\hline & \\
\hline & \\
\hline
\end{tabular}

All other medications: (list) 


\section{Section IV: Sitting habits during the working day}

Part A: In the 4 weeks prior to onset of symptoms that led to presentation:

If you consider your time at work and for work-related activities only:

1. What is the total amount of time you usually spent sitting in any one day?

2. What is the maximum total amount of time you spent sitting in any one day?

3. No of days with $\geq 10$ hours sitting at work $(A)$ :

\section{Part B: In the 4 weeks prior to onset of symptoms that led to presentation:}

If you consider your time outside work in front of a computer for recreational purposes:

1. What is the total amount of time you usually spent sitting in any one day?

2. What is the maximum total amount of time you spent sitting in any one day?

3. No of days with $\geq 10$ hours sitting for combination of work and on the computer $(A+B)$ :

Part C: In the 4 weeks prior to onset of symptoms that led to presentation: If you consider your time travelling to and from work:

1. What is the total amount of time you usually spent sitting in any one day?

2. What is the maximum total amount of time you spent sitting in any one day?

3. No of days with $\geq 10$ hours sitting for combination of work, computer and travelling $(A+B+C)$ :

\section{Part D: In the 4 weeks prior to onset of symptoms that led to presentation:}

Did you spend any time using a laptop positioned on your knees or lap?

If yes,

1. What is the total amount of time you usually spent doing this in any one day?

2. What is the maximum total amount of time you spent doing this in any one day? 


\section{Part E: In the 4 weeks prior to onset of symptoms that led to presentation:}

1. What is the usual amount of time you spent sitting At work + work related computer use (A): at any one time without getting up?

Outside work for recreational computer use (B):

Travelling to / from work (C):

Using laptop (D):

2. What is the maximum time you spent sitting at any At work + work related computer use (A): one time without getting up?

Outside work for recreational computer (B):

Travelling to / from work (C):

Using laptop (D)

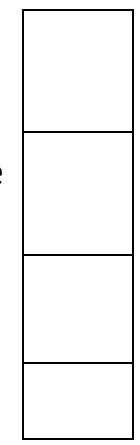

\section{Section V: Office structure}

1. Do you have a specific desk and chair at work?

2. Is your chair adjustable?

3. Can you stretch out your legs fully while seated?

4. Do you work in an office environment for more than $50 \%$ of your working time?

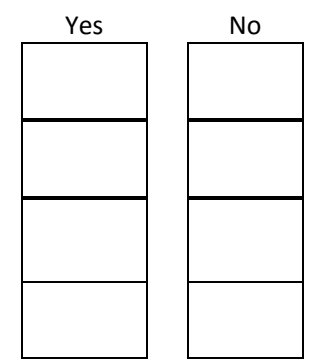

5. Do you eat lunch at your work desk?
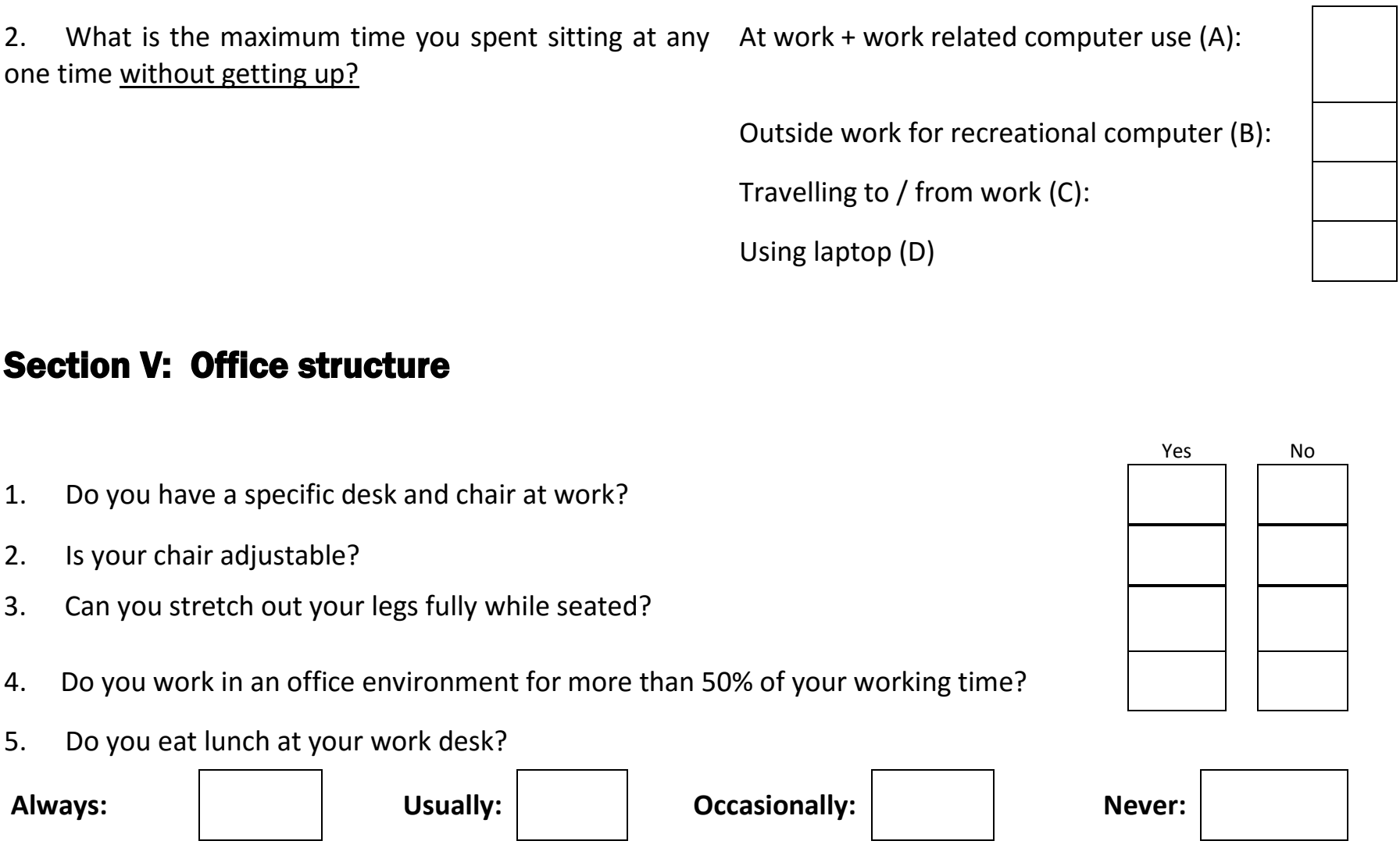
7. Do you stand up for prolonged periods without moving around? (i.e. more than $1 \mathrm{hr}$ )

8. Do you stand, on average, for more than 6 hours/day, at work?

If yes please specify:

\section{Section Vl: Preventative strategies}

In the 4 weeks prior to onset of symptoms that led to presentation:

Hospital based prophylaxis

Were you seen in a hospital setting? If yes,

Was the risk of VTE discussed with you?

Were you recommended or prescribed any of the following as VTE prevention measures? Situation for which VTE measure given:

\begin{tabular}{|c|c|c|c|}
\hline \multirow[b]{2}{*}{ Aspirin } & Yes & No & Comment \\
\hline & & & \\
\hline Low molecular weight heparin & & & \\
\hline Graduated compression or TED stockings & & & \\
\hline Mechanical & & & \\
\hline Other VTE prevention method & & & \\
\hline
\end{tabular}




\section{Section VIl: Clinical detalls regarding the severity of the VTE event}

Diagnosis:

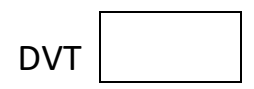

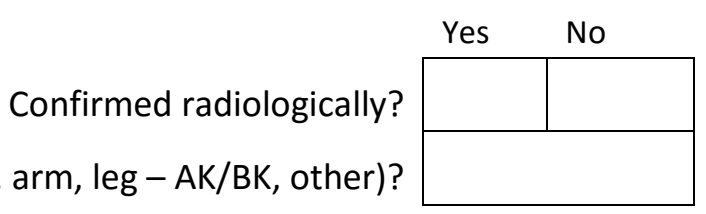

Diagnosis:

PE

For cases involving PE:

Presumed (clinically suggestive but no radiological confirmation performed)

Confirmed by CTPA or VQ scan

Submassive (large thrombus with Trop T rise or evidence of RVstrain)

Massive (large thrombus on CTPA with syncope or BP $<90$ syst)

Did the patient receive thrombolysis?

\section{Risk factor recognition:}

DVT location (e.g. arm, leg - AK/BK, other)?
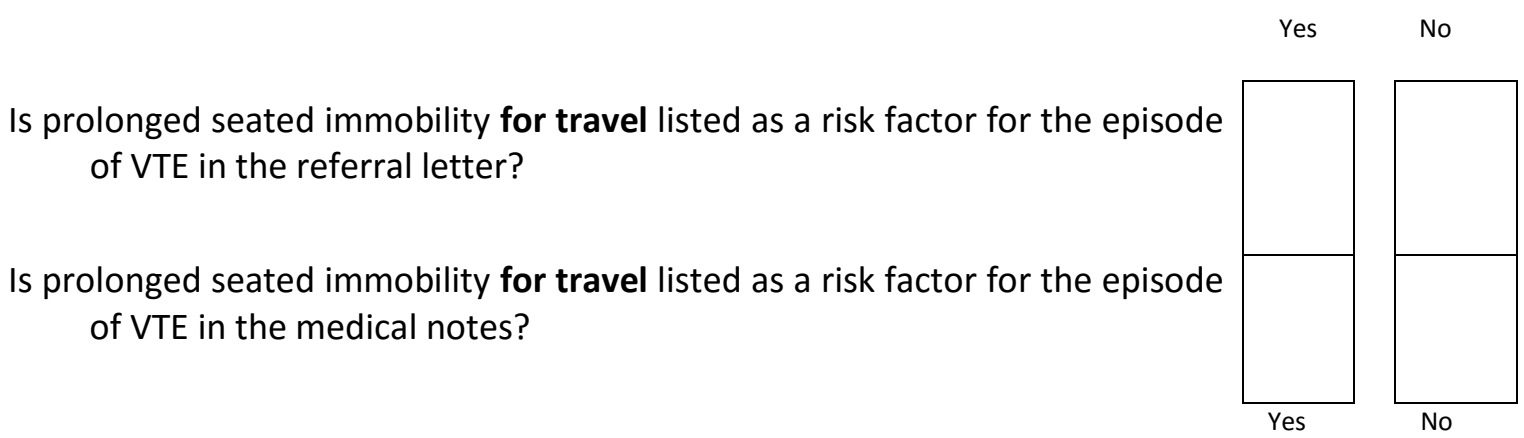

NA

Is prolonged seated immobility at work listed as a risk factor for VTE in the referral letter?

Is prolonged seated immobility at work listed as a risk factor for VTE in the medical notes?

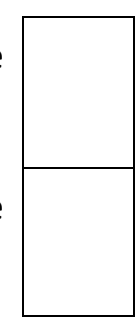

Yes

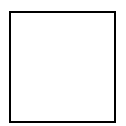

Yes No

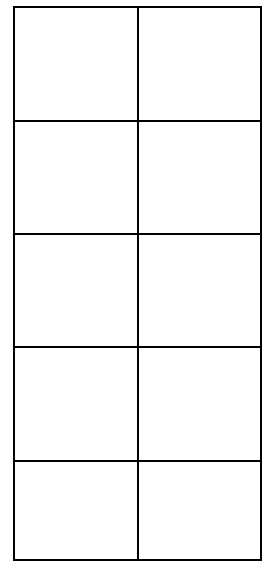

No
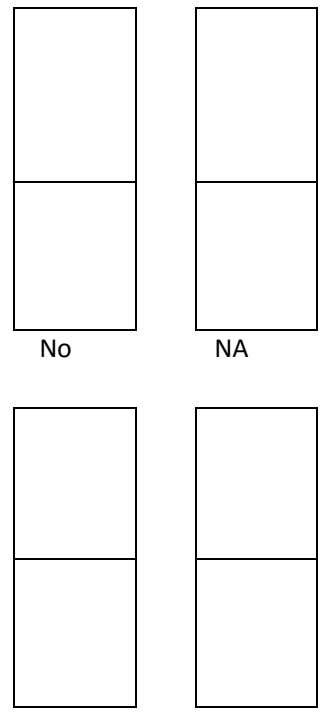

No
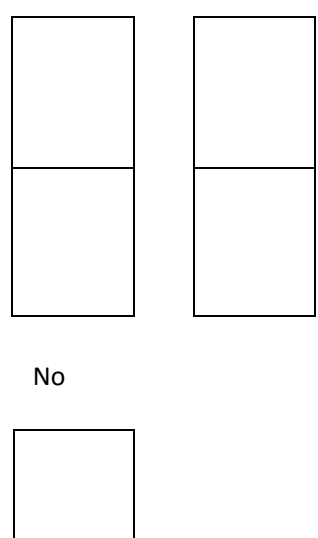

Was this event considered an idiopathic (unprovoked) event?

Section VIII: Thrombophilia screen (to be completed once blood results available) 
Tick the appropriate option:

Not done or results not performed through CCDHB laboratories

Thrombophilia screen negative

Thrombophilia screen abnormal

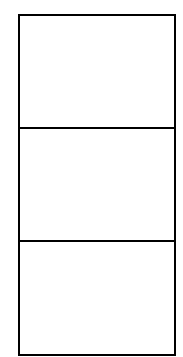

If abnormal, select from the following:

AT III

Protein C deficiency

Protein S deficiency

Factor V Leiden

Prothrombin gene

Antiphospholipid antibodies

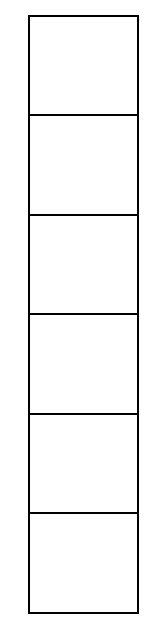

Investigator signature and date: 
Appendix III: Venous thromboembolism risk associated with protracted work- and computer-related seated immobility: A case-control study. Questionnaire: Controls 


\section{SIT 3 questionnaire - Controls}

Date :

\section{Section I: Demographic detalls}

Name:

Hospital Number:

Ethnicity

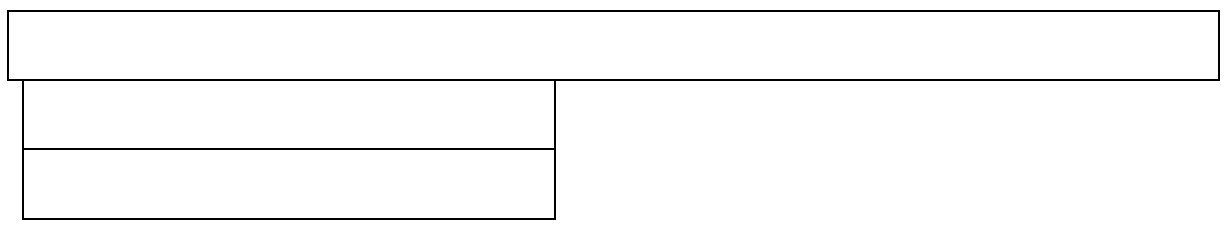

Date Of Birth:

Gender:

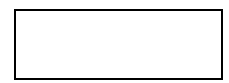

Weight:

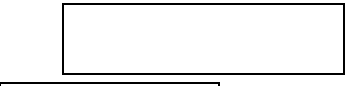

Height:

Occupation (at date of onset of symptoms):

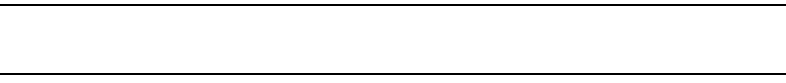

ANSCO Occupation code (11-94)

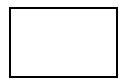

\section{Section II: Orthopaedic Detalls}

Date of onset of symptoms/injury:

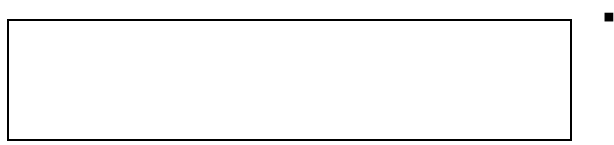

Date of presentation:

\section{Admitted to hospital? YES/ NO}

Diagnosis leading to emergency department presentation (eg: fracture, dislocation, other):

Site of injury (eg: radius, shoulder / left, right) 
Injury context (work, recreational, other)

\section{Section III: VTE risk factors}

Have you had a VTE in last 6 months?

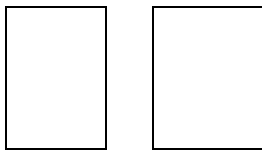

Previous history of VTE - specify:

1.

2.

Family history of VTE - specify:
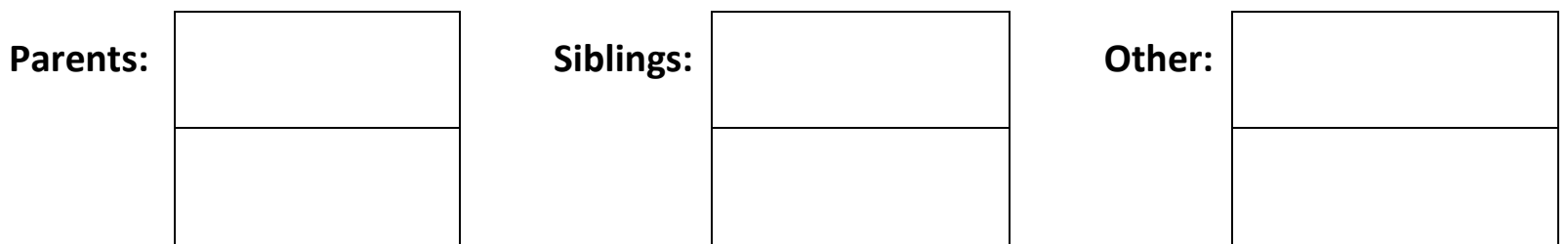

Part A: In the 4 weeks prior to onset of symptoms that led to presentation: 
Long distance travel ( $\geq 4 \mathrm{hrs}$ ):

\begin{tabular}{|c|c|c|c|}
\hline & Time before & Duratio & f travel \\
\hline & $\begin{array}{l}\text { onset of } \\
\text { symptoms } \\
\text { (days) }\end{array}$ & $\begin{array}{l}\text { Max. hours } \\
\text { per sector }\end{array}$ & $\begin{array}{c}\text { Total } \\
\text { Duration (hrs) }\end{array}$ \\
\hline Car & & & \\
\hline International air & & & \\
\hline Other (specify) & & & \\
\hline
\end{tabular}


Part B: In the 4 weeks prior to onset of symptoms that led to presentation:

Other risk factors:

Surgery

Other immobility (e.g.Hospital admission, drug overdose, , wheelchair bound, bedrest) $\geq 2$ days

Cast/plaster and/or traumatic injury

Pregnancy or hormonal treatment

Malignancy or chronic disease

Smoking

History of varicose veins
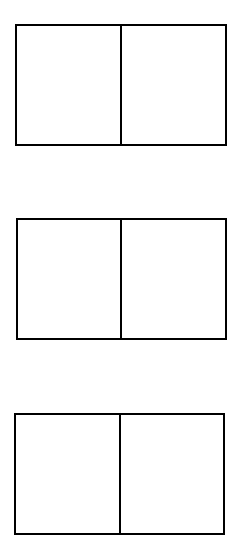

Yes No Details
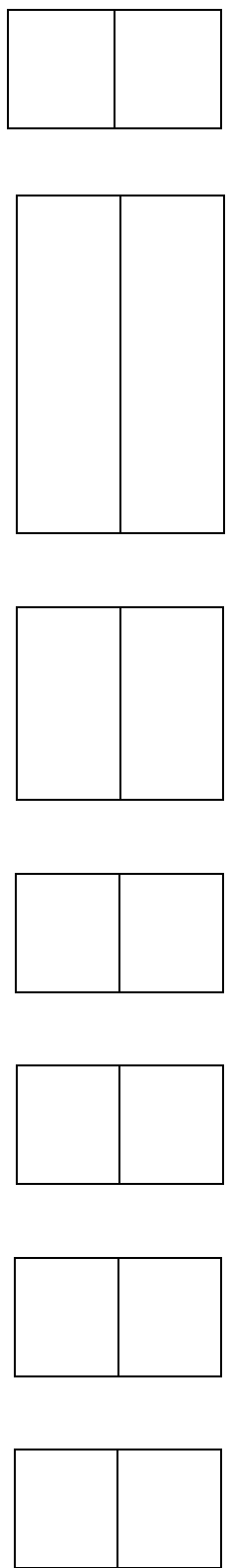
Part C: In the 4 weeks prior to onset of symptoms that led to presentation:

Details of the form of exercise

Strenuous exercise ( $\geq 30$ mins):

Specify whether road or MTB gym equipment etc

Cycling

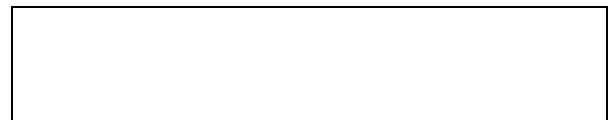

Frequency and duration of exercise No of Longest times duration (hrs)
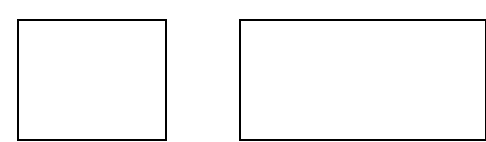

Rowing / kayaking / waka ama
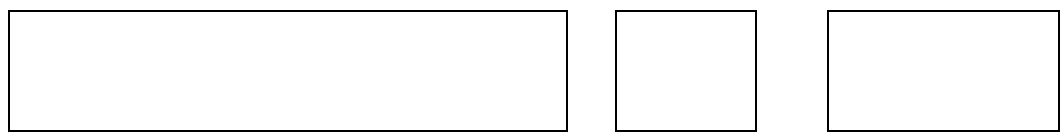

Weights (i.e. squats, lunges)
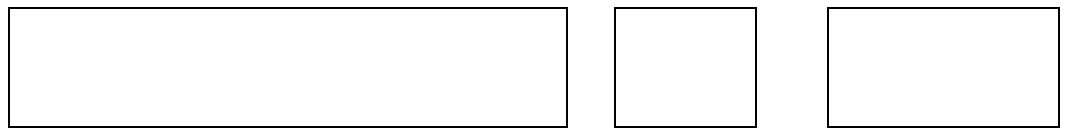

Long distance running
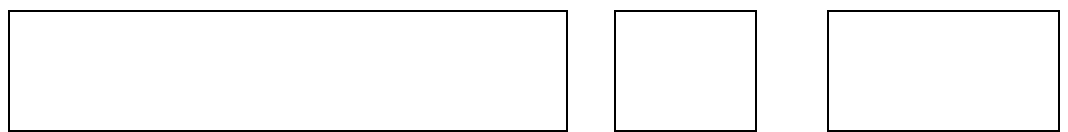

Other strenuous exercise

Note: "strenuous exercise" is defined as: cardiovascular or weight related exercise that involves repetitive physical movements 


\section{Section IV: Medications}

In the 4 weeks prior to onset of symptoms that led to presentation:

Anti-thrombotic agents Yes No Details:

Enoxaparin

Warfarin

Aspirin

Clopidogrel

Dipyridamole

Other

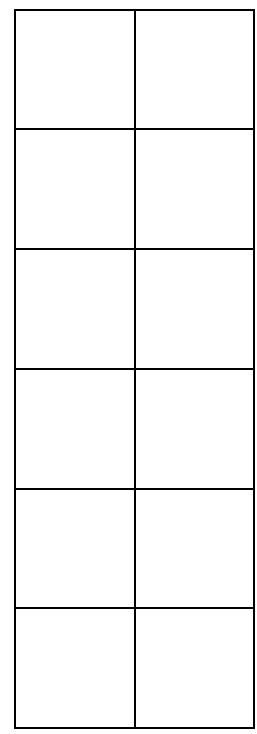

Yes No Details of treatment i.e. tamoxifen, anastrozole, chemotherapy etc

\section{Pro-thrombotic agents}

Anti-cancer medications

HRT

OCP

Tranexamic acid

Other

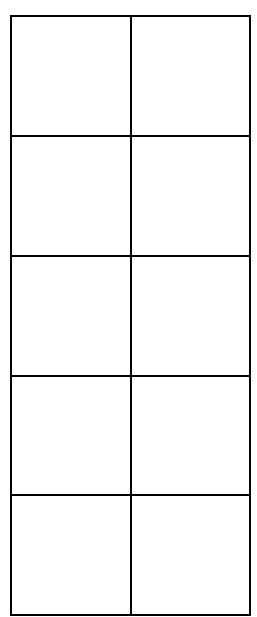


All other medications: (list)

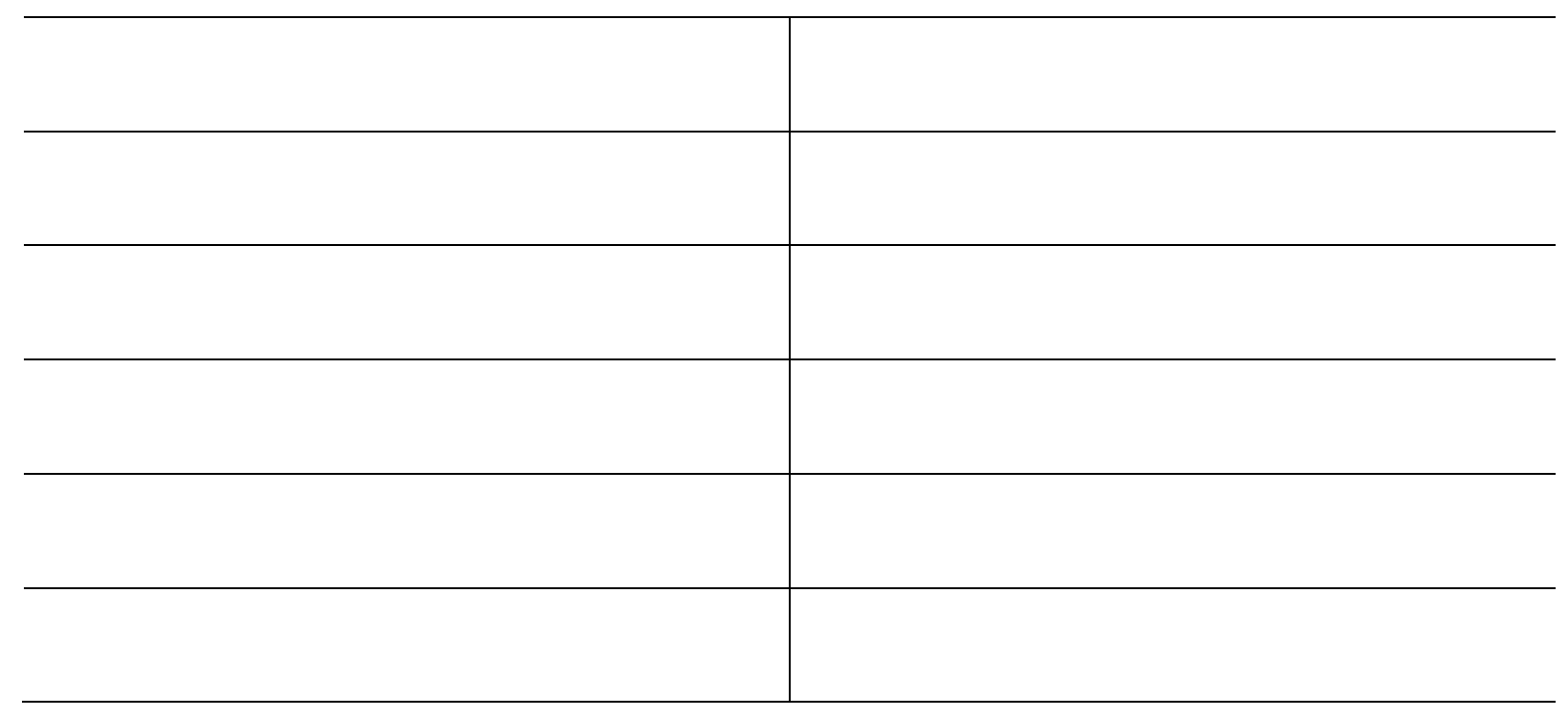




\section{Section V: Sitting habits during the working day}

Part A: In the 4 weeks prior to onset of symptoms that led to presentation

If you consider your time at work and for work related activities only:

1. What is the total amount of time you usually spent sitting in any one day?

2. What is the maximum total amount of time you spent sitting in any one day?

3. No of days with $\geq 10$ hours sitting at work $(A)$ :

\section{Part B: In the 4 weeks prior to onset of symptoms that led to presentation}

If you consider your time spent outside work on the computer for recreational activites:

4. What is the total amount of time you usually spent sitting in any one day?

5. What is the maximum total amount of time you spent sitting in any one day?

6. No of days with $\geq 10$ hours sitting for combination of work and on the computer $(A+B)$ :

\section{Part C: In the 4 weeks prior to onset of symptoms that led to presentation}

If you consider your time spent travelling to and from work: 
1. What is the total amount of time you usually spent sitting in any one day?

2. What is the maximum total amount of time you spent sitting in any one day?

3. No of days with $\geq 10$ hours sitting for combination of work, computer and travelling $(A+B+C)$ :

Part D: In the 4 weeks prior to onset of symptoms that led to presentation:

Did you spend any time using a laptop positioned on your knees or lap?

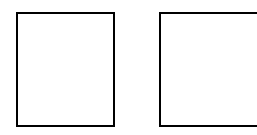
If yes,

1. What is the total amount of time you usually spent doing this in any one day?

2. What is the maximum total amount of time you spent doing this in any one day?

\section{Part E: In the 4 weeks prior to onset of symptoms that led to presentation}

1. What is the usual amount of time you spent sitting at any one time without getting up?
At work and for work related computer use $(A)$ :

Outside work for recreational computer use (B):

Travelling to / from work (C): 
Using Laptop (D):

2. What is the maximum time you spent sitting at any one At work and for work related time without getting up? computer use $(A)$ :

Outside work for recreational computer use (B):

Travelling to / from work (C):

Using laptop 


\section{Section Vl: Office structure}

Yes

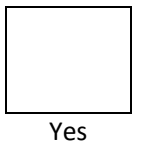

Yes
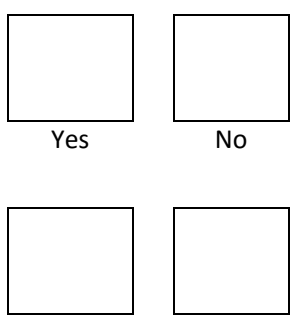

4. Do you work in an office environment for more than $50 \%$ of your working time?

5. Do you eat lunch at your work desk?

Always:
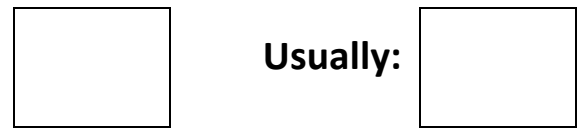

Occasionally:

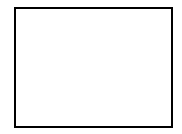

Never:
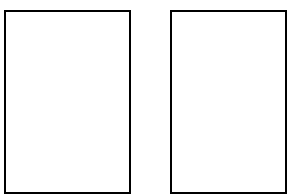

6. Do you have a computer reminder on your work computer, to get up periodically from being seated?

(select only one option)

(a) I don't have a specific work computer 
(b) I have a specific work computer but am not aware of having a computer reminder installed on it

(c) My work computer which has a computer reminder, but I tend to ignore it, or it has been turned off

(d) I have a computer reminder and I usually get up when reminded to do so

If yes: how frequently does the reminder prompt you to get

up? mins

6. Do you stand up for prolonged periods without moving around? (i.e. more than $1 \mathrm{hr}$ )

7. Do you stand, on average, for more than 6 hours/day at work?
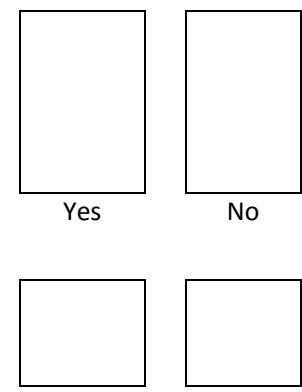

If yes please specify:

Investigators signature:

Date: 
Appendix IV: Australian and New Zealand Classification of Occupations (ANZSCO): Level 2 Occupations 
1 MANAGERS

11 Chief Executives, General Managers and Legislators

12 Farmers and Farm Managers

13 Specialist Managers

14 Hospitality, Retail and Service Managers

2 PROFESSIONALS

21 Arts and Media Professionals

22 Business, Human Resource and Marketing Professionals

23 Design, Engineering, Science and Transport Professionals

24 Education Professionals

25 Health Professionals

26 ICT Professionals

27 Legal, Social and Welfare Professionals

3 TECHNICIANS AND TRADES WORKERS

31 Engineering, ICT and Science Technicians

32 Automotive and Engineering Trades Workers

33 Construction Trades Workers

34 Electrotechnology and Telecommunications Trades Workers

35 Food Trades Workers

36 Skilled Animal and Horticultural Workers

39 Other Technicians and Trades Workers

4 COMMUNITY AND PERSONAL SERVICE WORKERS

41 Health and Welfare Support Workers

42 Carers and Aides

43 Hospitality Workers

44 Protective Service Workers

45 Sports and Personal Service Workers

5 CLERICAL AND ADMINISTRATIVE WORKERS

51 Office Managers and Program Administrators

52 Personal Assistants and Secretaries

53 General Clerical Workers

54 Inquiry Clerks and Receptionists

55 Numerical Clerks

56 Clerical and Office Support Workers

59 Other Clerical and Administrative Workers

6 SALES WORKERS

61 Sales Representatives and Agents

62 Sales Assistants and Salespersons

63 Sales Support Workers

7 MACHINERY OPERATORS AND DRIVERS

71 Machine and Stationary Plant Operators

72 Mobile Plant Operators 
73 Road and Rail Drivers

74 Storepersons

8 LABOURERS

81 Cleaners and Laundry Workers

82 Construction and Mining Labourers

83 Factory Process Workers

84 Farm, Forestry and Garden Workers

85 Food Preparation Assistants

89 Other Labourers 
Appendix V: Participant information sheet: Legflow: Haemodynamic study 
You are invited to take part in a study on blood flow in the leg when seated. Whether or not you take part is your choice. If you don't want to take part, you don't have to give a reason, and it won't affect the care you receive. If you do want to take part now, but change your mind later, you can pull out of the study at any time.

This Participant Information Sheet will help you decide if you'd like to take part. It sets out why we are doing the study, what your participation would involve, what the benefits and risks to you might be, and what would happen after the study ends. We will go through this information with you and answer any questions you may have. You do not have to decide today whether or not you will participate in this study. Before you decide you may want to talk about the study with other people, such as family, whānau, friends, or healthcare providers. Feel free to do this.

If you agree to take part in this study, you will be asked to sign the Consent Form on the last page of this document. You will be given a copy of both the Participant Information Sheet and the Consent Form to keep. We can arrange for an interpreter if you need one.

This document is 7 pages long, including the Consent Form. Please make sure you have read and understood all the pages.

\section{What is the purpose of the study?}

We want to see whether using a special foot-stand while you are sitting down helps improve blood flow in the legs. We know that people sitting down for long periods of time (like when they are flying long distances, or at a job that requires lots of sitting), may be at increased risk of developing blood clots called deep vein thrombosis (DVT) in their legs. This risk might be partly due to pooling of blood in the leg during long periods of sitting. Using this foot-stand may help reduce pooling by improving the return of blood from the legs while you are sitting down.

To see if this works, we want to measure the return of blood from the legs in study subjects while they are sitting down. One leg will be using the foot-stand device every so often, and the other leg will be held still. We will compare the rate of flow of blood in the leg of the moving foot to the rate 
of flow of blood in the leg of the in the stable foot. Whichever foot uses the foot-stand device will be randomized (allocated by chance), so there will be a $50 \%$ chance that you will be asked to use the footstool with your left foot and a $50 \%$ chance that you will use the footstool with your right foot.

If we can show that blood flow is improved when using this foot-stool, we may be able to develop a simple and safe tool for people to help reduce the risk of developing blood clots when they are sitting down for long periods of time.

This study is funded by the Medical Research Institute of New Zealand. If you have any questions about the study, you may contact the study investigator:

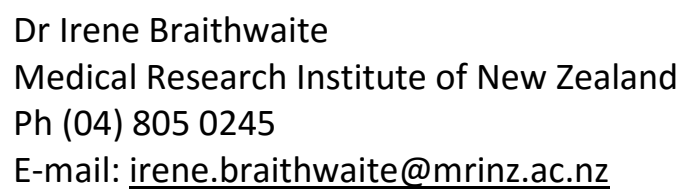

This study has been approved by the Victoria University Human Ethics Committee.

\section{What will my participation in the study involve?}

If you are generally healthy, are not pregnant, aged between 18 and 65 years, and have no current or previous history of blood vessel or muscular problems in your legs, you may be eligible for this study.

Participation will involve two separate visits. At the first visit (about 20 minutes long) you will see research staff at the Medical Research Institute of New Zealand to undertake an informed consent, a brief medical history and to examine your legs to make sure there are no problems that will exclude you from the study. We will also show you how to use the foot-rest to ensure you are able to participate in the study.

You will then be asked to attend an appointment at Pacific Radiology at 668 High Street, Lower Hutt to undertake the study procedures;

- You will be randomised (like tossing a coin), to use the foot-rest device with either your right foot or your left foot. You will have a 50\% chance of being allocated to the right foot group or the left foot group.

- We will ask you to sit quietly for 10 minutes on a seat, with both feet placed on a platform which has been adjusted to suit your height. 
- At the end of that 10 minute period we will do an ultrasound (USS) measurement of the veins in the back of both your knees to measure how fast blood is returning from your feet to your heart. This is called the baseline measurement. (A USS is the use of sound waves to see blood vessels and other internal body structures without having to use needles, surgery or other invasive procedures. You may have seen USS used during pregnancy scans. It is considered to have very little if any risks for patients).

- After the baseline USS has been done, we will place your allocated foot on the foot-rest while keeping the other foot placed on the platform. At 14 minutes we will take two measurements of the blood flow in the back of your foot while it is moving over the footstool, and then immediately after getting these two measures we will ask you to stop and we will measure the blood flow in the back of your immobile leg.

- We will repeat the measurements again at 18 minutes.

The study visit should take approximately 25 minutes, but it is possible there may be delays if other participants run late or if we have technical difficulties.

Please note: We ask that all participants do not undertake strenuous physical activities within 24 hours of their ultrasound scan appointment, as this may influence vein blood flow or cause vein injury. This includes running, cycling and lifting weights involving squats.

We recommend that all participants wear or bring a pair of shorts with them to change into, as this makes it easier to ultrasound your leg.

If you wish to have family / whanau present while you attend either or both appointments, feel free to do this.

\section{What are the possible benefits and risks of this study?}

You may find the use of the foot-stool slightly awkward initially, but this should not be painful. If you experience any pain or significant discomfort/distress, or if the researchers are concerned about your wellbeing during the trial, you may be withdrawn from the trial.

In order to measure the blood flow in your legs using ultrasound equipment it is necessary to apply a viscous gel to the skin for the duration of each measurement. In some individuals with sensitive skin there is a small possibility of minor skin irritation from the use of this gel. 
There are no other anticipated risks from participating in this study.

\section{Who pays for the study?}

We do not anticipate that you will incur any costs associated with taking part in this study. We will provide reimbursement of $\$ 50$ to try and cover any costs or inconvenience that may have been incurred as a result of you participating.

\section{What if something goes wrong?}

If you were injured in this study, which is unlikely, you would be eligible for compensation from ACC just as you would be if you were injured in an accident at work or at home. You will have to lodge a claim with ACC, which may take some time to assess. If your claim is accepted, you will receive funding to assist in your recovery.

If you have private health or life insurance, you may wish to check with your insurer that taking part in this study won't affect your cover.

\section{What are my rights?}

You have the ability to see any information collected about you during the study, and if you request, a researcher will be available to provide explanation, or to answer any questions you may have relating to that information.

All information collected about you will be stored confidentially in secure offices. You will not be able to be identified individually in any published study results.

\section{What happens if I change my mind and withdraw from the study?}

Your participation in this study is voluntary. You can choose not to participate at all, or withdraw at any time even after you have given your consent and started the study. Choosing not to participate, or choosing to withdraw after you have started the study will not affect your future healthcare.

If you decide to withdraw from the study, you may do so at any time, you just need to let me know at the time. Any information collected about you up to the point when you withdraw may continue to be used in the study.

\section{What happens after the study?}

The foot-rest device being tested is not commercially available and will not be available after the study has concluded. 
Data gathered during this study, including any of your own personal information will be securely stored for 15 years by the MRINZ to meet our ethical and Good Clinical Practice responsibilities. After this period of time, the records will be destroyed to maintain confidentiality and privacy of all participants.

It is possible we may use some of the data from this study to contribute to future studies. This would comprise ultrasound measurement results and other non-identifiable data.

You have the opportunity to tell us if you would like a copy of the final results of this study during the informed consent process. This should be able to be done within approximately 6 months. The MRINZ aims to have all studies written up and published in reputable journals, and to present our findings in medical and scientific forums. You will not be able to be personally identified in any of these publications or presentations.

\section{Who do I contact for more information or if I have concerns?}

If you have any questions, concerns or complaints about the study at any stage, you can contact:

Dr Irene Braithwaite

Senior Research Fellow

Medical Research Institute of New Zealand

(04) 8050245

Irene.braithwaite@mrinz.ac.nz

If you want to talk to someone who isn't involved with the study, you can contact an independent health and disability advocate on:

Phone: 0800555050

Fax: $\quad 08002$ SUPPORT (0800 2787 7678)

Email: $\quad$ advocacy@hdc.org.nz

For Maori health support please contact :

Whānau Care Services, Cultural Care Centre, Level 2, Wellington Hospital

Phone: $\quad 0800999442$ or 048060948

Email: $\quad$ wcs@ccdhb.org.nz

You can also contact the Victoria University Human Ethics Committee that approved this study on:

Phone: $\quad$ (04) 4635620

Email: $\quad$ research-office@vuw.ac.nz 


\section{Consent Form}

\section{If you need an INTERPRETER, please tell us.}

\section{All of the 'Yes' boxes must be ticked to be able to progress on to the study.}

I have read, or have had read to me in my first language, and I understand the Participant Information Sheet.

\begin{tabular}{l}
\hline I have been given sufficient time to consider whether or not to participate in this study. \\
\hline I have had the opportunity to use a legal representative, whanau/ family support or a \\
friend to help me ask questions and understand the study. \\
$\begin{array}{l}\text { I am satisfied with the answers I have been given regarding the study and I have a copy of } \\
\text { this consent form and information sheet. }\end{array}$ Yes $\square$ \\
\hline I understand that taking part in this study is voluntary (my choice) and that I may \\
withdraw from the study at any time without this affecting my medical care. \\
\hline I consent to the research staff collecting and processing my information, including \\
information about my health. \\
If I decide to withdraw from the study, I agree that the information collected about me up \\
to the point when I withdraw may continue to be processed. \\
\hline I consent to my GP or current provider being informed about my participation in the \\
study and of any significant abnormal results obtained during the study.
\end{tabular}

I agree to an approved auditor appointed by the New Zealand Health and Disability Ethic Committees, or any relevant regulatory authority or their approved representative reviewing my relevant medical records for the sole purpose of checking the accuracy of the information recorded for the study.

I understand that my participation in this study is confidential and that no material, which could identify me personally, will be used in any reports on this study.

Yes $\square$

\begin{tabular}{lr}
\hline I understand the compensation provisions in case of injury during the study. & Yes $\square$ \\
\hline I know who to contact if I have any questions about the study in general. & Yes $\square$ \\
\hline I understand my responsibilities as a study participant. & Yes $\square$ \\
\hline I wish to receive a summary of the results from the study. & Yes $\square$
\end{tabular}


Declaration by participant:

I hereby consent to take part in this study.

Participant's name:

Signature: Date:

Declaration by member of research team:

I have given a verbal explanation of the research project to the participant, and have answered the participant's questions about it.

I believe that the participant understands the study and has given informed consent to participate.

Researcher's name:

Signature:

Date: 
Appendix VI: Participant Information Sheet: The effect of lower limb fibreglass cast on the efficacy of the Impulse IPC device on lower limb venous haemodynamics in healthy subjects 
Locality: Medical Research Institute of New Zealand
Ethics committee ref.: 14 STH 82

Contact phone number: (04) 8050245

You are invited to take part in a study investigating the effect of intermittent compression devices and fibreglass casts on blood flow in the leg. Whether or not you take part is your choice. If you don't want to take part, you don't have to give a reason, and it won't affect the care you receive. If you do want to take part now, but change your mind later, you can pull out of the study at any time.

This Participant Information Sheet will help you decide if you'd like to take part. It sets out why we are doing the study, what your participation would involve, what the benefits and risks to you might be, and what would happen after the study ends. We will go through this information with you and answer any questions you may have. You do not have to decide today whether or not you will participate in this study. Before you decide you may want to talk about the study with other people, such as family, whānau, friends, or healthcare providers. Feel free to do this.

If you agree to take part in this study, you will be asked to sign the Consent Form on the last page of this document. You will be given a copy of both the Participant Information Sheet and the Consent Form to keep. We can arrange for an interpreter if you need one.

This document is 7 pages long, including the Consent Form. Please make sure you have read and understood all the pages.

\section{What is the purpose of the study?}

There is a device called an Intermittent Pneumatic Compression (IPC) device, that may be used to improve blood flow in the legs of patients when they have had operations or been admitted to hospital. IPCs have been shown to reduce the risk of developing blood clots in the legs (Deep Vein Thrombosis - DVT) and the lungs (Pulmonary Embolism - PE) due to long periods of immobility. They work by placing an inflatable cuff on the patients' calf or foot, which is then inflated several times a minute while the patient is immobilised. The inflated cuff squeezes the veins in the sole of the foot 
and the calf, reducing pooling of blood in the leg, and thus reducing the risk of blood clots forming in the veins.

We want to see whether the fibreglass cast impairs the performance of the IPC underneath it.

To figure this out, we want to measure the return of blood from the legs in study participants who have an IPC device on both feet, and who then have one leg placed in a fibreglass cast. We want to measure blood flow at the following time points:

- After you have been sitting for 10 minutes and before any IPC's or casts are placed on your leg

- After the IPCs have been placed on both your legs and one of your legs has also been placed in a cast

- $\quad$ After both IPCs have been running for 10 minutes

The measurement will be done using an Ultrasound machine, and the measurements will be made at the big veins that run up behind your knees (the popliteal veins). An ultrasound (USS) is the use of sound waves to see blood vessels and other internal body structures without having to use needles, surgery or other invasive procedures. You may have seen USS used during pregnancy scans. It is considered to have very little if any risks for patients.

Whichever leg goes in the fibreglass cast will be randomized (allocated by chance), so there will be a $50 \%$ chance that your left leg will go in a cast and a 50\% chance that your right leg will go in a cast.

If we can show that the performance of the IPC device is not impaired by being placed under a fibreglass cast, this may become a safe and reliable tool to help reduce the risk of developing blood clots when people have had their lower leg immobilised.

This study is funded by the Medical Research Institute of New Zealand. If you have any questions about the study, you may contact the study investigator:

Dr Irene Braithwaite

Medical Research Institute of New Zealand

$\mathrm{Ph}(04) 8050245$

E-mail: irene.braithwaite@mrinz.ac.nz 
This study has been approved by the Health and Disability Ethics Committee: 14 STH 82 /

03/07/2014

\section{What will my participation in the study involve?}

If you are generally healthy, are not pregnant, aged between 18 and 65 years, and have no current or previous history of blood vessel or muscular problems in your legs, you may be eligible for this study.

Participation will involve two separate visits. At the first visit (about 20 minutes long) you will see research staff at the Medical Research Institute of New Zealand to complete an informed consent, a brief medical history and to examine your legs to make sure there are no problems that will exclude you from the study.

You will then be asked to attend an appointment at Pacific Radiology at 668 High Street, Lower Hutt to undertake the study procedures;

- We will ask you to sit quietly on an examination bed for 10 minutes

- At the end of that 10 minute period we will do a USS measurement of the popliteal veins to see how fast blood is returning from your feet to your heart. This is called the baseline measurement.

- After the USS has been done, we will put inflatable foot pads on both your feet and hook them up to the 'pump' device so the foot pads can be inflated.

- We will randomise you to have either your left or right lower leg placed in a fibreglass cast.

- We will then ask you to sit quietly on the examination bed for approximately another 20 minutes, after which we will do a further USS measure of blood flow in the popliteal veins.

- We will then turn on both the IPC devices.

- After 10 minutes of the IPC devices running we will do USS measurements of popliteal blood flow again.

The study visit should take approximately 80 minutes, but it is possible there may be delays if other participants run late or if we have technical difficulties. 
Please note: We ask that all participants do not undertake strenuous physical activities 24 hours prior to their ultrasound scan appointment, as this may influence vein blood flow or cause vein injury. This includes running, cycling and lifting weights involving squats.

We recommend that all participants wear or bring a pair of shorts with them to change into, as this makes it easier to ultrasound your leg.

If you wish to have family / whanau present while you attend either or both appointments, feel free to do this.

\section{What are the possible benefits and risks of this study?}

You may find the compression devices slightly uncomfortable when they are first turned on, this will quickly settle. The cast will be placed by experienced fracture clinic staff and will not be on for more than approximately 60 minutes and they are easily removed. If you experience any pain, significant discomfort/distress, or if the researchers are concerned about your wellbeing during the trial, you may be withdrawn from the trial.

In order to measure the blood flow in your legs using ultrasound equipment it is necessary to apply a viscous gel to the skin for the duration of each measurement. In some individuals with sensitive skin there is a small possibility of minor skin irritation from the use of this gel.

There are no other anticipated risks from participating in this study.

\section{Who pays for the study?}

We do not anticipate that you will incur any costs associated with taking part in this study. We will provide reimbursement of $\$ 100$ to try and cover any costs or inconvenience that may have been incurred as a result of you participating.

\section{What if something goes wrong?}

If you were injured in this study, which is unlikely, you may be eligible for compensation from ACC just as you would be if you were injured in an accident at work or at home. You will have to lodge a claim with ACC, which may take some time to assess. If your claim is accepted, you will receive funding to assist in your recovery.

If you have private health or life insurance, you may wish to check with your insurer that taking part in this study won't affect your cover. 


\section{What are my rights?}

Your participation in this study is voluntary. You can choose not to participate at all, or withdraw at any time even after you have given your consent and started the study. Choosing not to participate, or choosing to withdraw after you have started the study will not affect your future healthcare.

You have the ability to see any information collected about you during the study, and if you request, a researcher will be available to provide explanation, or to answer any questions you may have relating to that information. All information collected about you will be stored confidentially in secure offices. You will not be able to be identified individually in any published study results.

\section{What happens after the study or if I change my mind?}

Data gathered during this study, including any of your own personal information will be securely stored for 15 years by the MRINZ to meet our ethical and Good Clinical Practice responsibilities. After this period of time, the records will be destroyed to maintain confidentiality and privacy of all participants.

It is possible we may use some of the data from this study to contribute to future studies. This would comprise ultrasound measurement results and other non-identifiable data.

You have the opportunity to tell us if you would like a copy of the final results of this study during the informed consent process. This should be able to be done within approximately 6 months. The MRINZ aims to have all studies written up and published in reputable journals, and to present our findings in medical and scientific forums. You will not be able to be personally identified in any of these publications or presentations.

\section{Who do I contact for more information or if I have concerns?}

If you have any questions, concerns or complaints about the study at any stage, you can contact:

Dr Irene Braithwaite

Senior Research Fellow

Medical Research Institute of New Zealand

(04) 8050245

Irene.braithwaite@mrinz.ac.nz 
If you want to talk to someone who isn't involved with the study, you can contact an independent health and disability advocate on:

Phone: 0800555050

Fax: $\quad 08002$ SUPPORT (0800 2787 7678)

Email: $\quad$ advocacy@hdc.org.nz

For Maori health support please contact :

Whānau Care Services, Cultural Care Centre, Level 2, Wellington Hospital

Phone: $\quad 0800999442$ or 048060948

Email: $\quad$ wcs@ccdhb.org.nz 


\section{Consent Form}

If you need an INTERPRETER, please tell us.

\section{Please tick to indicate you consent to the following}

\begin{tabular}{|c|c|c|}
\hline $\begin{array}{l}\text { I have read, or have had read to me in my first language, and I understand } \\
\text { the Participant Information Sheet. }\end{array}$ & Yes $\square$ & No $\square$ \\
\hline $\begin{array}{l}\text { I have been given sufficient time to consider whether or not to participate } \\
\text { in this study. }\end{array}$ & Yes $\square$ & No $\square$ \\
\hline $\begin{array}{l}\text { I have had the opportunity to use a legal representative, whanau/ family } \\
\text { support or a friend to help me ask questions and understand the study. }\end{array}$ & Yes $\square$ & No $\square$ \\
\hline $\begin{array}{l}\text { I am satisfied with the answers I have been given regarding the study and } \\
\text { I have a copy of this consent form and information sheet. }\end{array}$ & Yes $\square$ & No $\square$ \\
\hline $\begin{array}{l}\text { I understand that taking part in this study is voluntary (my choice) and } \\
\text { that I may withdraw from the study at any time without this affecting my } \\
\text { medical care. }\end{array}$ & Yes $\square$ & No $\square$ \\
\hline $\begin{array}{l}\text { I consent to the research staff collecting and processing my information, } \\
\text { including information about my health. }\end{array}$ & Yes $\square$ & No $\square$ \\
\hline $\begin{array}{l}\text { If I decide to withdraw from the study, I agree that the information } \\
\text { collected about me up to the point when I withdraw may continue to be } \\
\text { processed. }\end{array}$ & Yes $\square$ & No $\square$ \\
\hline $\begin{array}{l}\text { I consent to my GP or current provider being informed about my } \\
\text { participation in the study and of any significant abnormal results obtained } \\
\text { during the study. }\end{array}$ & Yes $\square$ & No $\square$ \\
\hline
\end{tabular}

I agree to an approved auditor appointed by the New Zealand Health and Disability Ethic Committees, or any relevant regulatory authority or their approved representative reviewing my relevant medical records for the sole purpose of checking the accuracy of the information recorded for the Yes $\square \quad$ No $\square$ study.

I understand that my participation in this study is confidential and that no material, which could identify me personally, will be used in any reports on this study.

Yes $\square \quad$ No $\square$

I understand the compensation provisions in case of injury during the study.

Yes $\square \quad$ No $\square$




\begin{tabular}{lcc}
\hline I know who to contact if I have any questions about the study in general. & Yes $\square$ & No $\square$ \\
\hline I understand my responsibilities as a study participant. & Yes $\square$ & No $\square$ \\
\hline I wish to receive a summary of the results from the study. & Yes $\square$ & No $\square$
\end{tabular}

\section{Declaration by participant:}

I hereby consent to take part in this study.

Participant's name:

Signature: Date:

\section{Declaration by member of research team:}

I have given a verbal explanation of the research project to the participant, and have answered the participant's questions about it.

I believe that the participant understands the study and has given informed consent to participate.

Researcher's name: 
Appendix VII: Validation of Fitbit ${ }^{\circledR}$ Flex $^{T M}$ : Participant data 
Participant Tables for validation of Fitbit ${ }^{\circledast}$ Flex $^{T M}$ capturing sitting time at work.

The following tables represent the data captured from participants and their allocated Fitbit ${ }^{\circledR}$ Flex $^{\mathrm{TM}}$. The largest proportional over-estimation Fitbit ${ }^{\circledast}$ Flex $^{T M}$ categorised sedentary time was noted in participant five, where there were several five minute periods noted by the participant as active, in which there were less than 25 steps recorded for each five minute period. These all occurred in the early part of the day where the participant was mostly active. It is possible that the participant retrospectively filled out their diary and considered themselves to have been up on their feet for that entire period. The largest under-estimations of sedentary time occurred in participants seven and nine. Both of these participants had few episodes of activity throughout the day, and may have either retrospectively batched their time, omitting of forgetting short periods of time when they may have got up from their seat, or may have bene doing some vigorous activity while seated setting off the Fitbit ${ }^{\circledast}$ Flex $^{T M}$ algorithm and recording a step although they were not mobile. None of the volunteers documented rapid alternating five minute periods of sedentariness and activity, and this was also the case with the Fitbit ${ }^{\circledast}$ Flex $^{T M}$, thus the reliability of the device in this setting is not demonstrated. However, given this is a study of those in roles designated as sedentary work this is less likely to be problematic. For the purposes of obtaining objective measures of time spent sitting in the proposed study sample, the Fitbit ${ }^{\circledR}$ Flex $^{T M}$ appears a reasonable option. 
Participant One. Fitbit ${ }^{\circledR}$ Flex $^{\mathrm{TM}}$ worn between 9.50 AM and 3.50 PM. Green cells denote agreement between 'sitting time' noted in participant diary and zero steps recorded by Fitbit $^{\circledR}$ Flex $^{\mathrm{TM}}$, purple cells denote agreement between 'sitting time' noted in participant diary and $<25$ steps recorded in the same five minute period by the Fitbit ${ }^{\circledR}$ Flex $^{\mathrm{TM}}$. Uncoloured cells indicate agreement between 'active' time noted in participant diary and 25 steps or more recorded in the same five minute period by the Fitbit ${ }^{\circledast}$ Flex $^{\mathrm{TM}}$. Red cells indicate 'active time' noted in the participant diary and $<25$ steps recorded in the same five minute period on the Fitbit $^{\circledR}$ Flex $^{\mathrm{TM}}$. Blue cells denote 'inactive time noted in the participant diary and $\mathbf{2 5}$ steps or more recorded in the same five minute period by the Fitbit $^{\circledR}$ Flex $^{\mathrm{TM}}$.

\begin{tabular}{|c|c|c|c|c|c|}
\hline Time & Steps & Time & Steps & Time & Steps \\
\hline 9:50:00 a.m. & 33 & 12:00:00 p.m. & 0 & 2:00:00 p.m. & 21 \\
\hline 9:55:00 a.m. & 79 & 12:05:00 p.m. & 0 & 2:05:00 p.m. & 25 \\
\hline 10:00:00 a.m. & 54 & 12:10:00 p.m. & 0 & 2:10:00 p.m. & 0 \\
\hline 10:05:00 a.m. & 0 & 12:15:00 p.m. & 55 & 2:15:00 p.m. & 0 \\
\hline 10:10:00 a.m. & 0 & 12:20:00 p.m. & 19 & 2:20:00 p.m. & 0 \\
\hline 10:15:00 a.m. & 46 & 12:25:00 p.m. & 0 & 2:25:00 p.m. & 0 \\
\hline 10:20:00 a.m. & 27 & 12:30:00 p.m. & 39 & 2:30:00 p.m. & 65 \\
\hline 10:25:00 a.m. & 33 & 12:35:00 p.m. & 42 & 2:35:00 p.m. & 56 \\
\hline 10:30:00 a.m. & 0 & 12:40:00 p.m. & 0 & 2:40:00 p.m. & 98 \\
\hline 10:35:00 a.m. & 0 & 12:45:00 p.m. & 0 & 2:45:00 p.m. & 42 \\
\hline 10:40:00 a.m. & 0 & 12:50:00 p.m. & 0 & 2:50:00 p.m. & 0 \\
\hline 10:45:00 a.m. & 46 & 12:55:00 p.m. & 30 & 2:55:00 p.m. & 0 \\
\hline 10:50:00 a.m. & 0 & 1:00:00 p.m. & 35 & 3:00:00 p.m. & 0 \\
\hline 10:55:00 a.m. & 0 & 1:05:00 p.m. & 0 & 3:05:00 p.m. & 0 \\
\hline 11:00:00 a.m. & 0 & 1:10:00 p.m. & 50 & 3:10:00 p.m. & 44 \\
\hline 11:05:00 a.m. & 0 & 1:15:00 p.m. & 0 & 3:15:00 p.m. & 30 \\
\hline 11:10:00 a.m. & 0 & 1:20:00 p.m. & 0 & 3:20:00 p.m. & 21 \\
\hline 11:15:00 a.m. & 0 & 1:25:00 p.m. & 0 & 3:25:00 p.m. & 0 \\
\hline 11:20:00 a.m. & 0 & 1:30:00 p.m. & 0 & 3:30:00 p.m. & 0 \\
\hline 11:25:00 a.m. & 42 & 1:35:00 p.m. & 0 & 3:35:00 p.m. & 14 \\
\hline 11:30:00 a.m. & 20 & 1:40:00 p.m. & 0 & 3:40:00 p.m. & 46 \\
\hline 11:35:00 a.m. & 0 & 1:45:00 p.m. & 0 & 3:45:00 p.m. & 539 \\
\hline 11:40:00 a.m. & 47 & 1:50:00 p.m. & 0 & & \\
\hline 11:45:00 a.m. & 0 & 1:55:00 p.m. & 0 & & \\
\hline 11:50:00 a.m. & 0 & & & & \\
\hline 11:55:00 a.m. & 0 & & & & \\
\hline \multicolumn{4}{|c|}{ Sedentary time Fitbit ${ }^{\circledR}$ Flex $^{\mathrm{TM}}$ (0 - 24 steps) } & \multicolumn{2}{|l|}{235 mins } \\
\hline \multicolumn{4}{|c|}{ Sedentary time diary } & \multicolumn{2}{|l|}{240 mins } \\
\hline \multicolumn{4}{|c|}{ Inaccuracy (0 - 24 steps) } & \multicolumn{2}{|l|}{$+2.1 \%$} \\
\hline
\end{tabular}


Participant Two. Fitbit ${ }^{\circledR}$ Flex $^{\mathrm{TM}}$ worn between 10.45 AM and 4.50 PM. Green cells denote agreement between 'sitting time' noted in participant diary and zero steps recorded by Fitbit ${ }^{\circledR}$ Flex $^{\mathrm{TM}}$, purple cells denote agreement between 'sitting time' noted in participant diary and $<25$ steps recorded in the same five minute period by the Fitbit ${ }^{\circledast}$ Flex $^{\mathrm{TM}}$. Uncoloured cells indicate agreement between 'active' time noted in participant diary and 25 steps or more recorded in the same five minute period by the Fitbit $^{\circledR}$ Flex $^{\mathrm{TM}}$. Red cells indicate 'active time' noted in the participant diary and $<25$ steps recorded in the same five minute period on the Fitbit ${ }^{\circledR}$ Flex $^{\mathrm{TM}}$. Blue cells denote 'inactive time noted in the participant diary and 25 steps or more recorded in the same five minute period by the Fitbit ${ }^{\circledR}$ Flex $^{\mathrm{TM}}$.

\begin{tabular}{|c|c|c|c|c|c|}
\hline Time & Steps & Time & Steps & Time & Steps \\
\hline 10:45:00 a.m. & 0 & 1:00:00 p.m. & 0 & 3:00:00 p.m. & 143 \\
\hline 10:50:00 a.m. & 37 & 1:05:00 p.m. & 0 & 3:05:00 p.m. & 199 \\
\hline 10:55:00 a.m. & 16 & 1:10:00 p.m. & 19 & 3:10:00 p.m. & 75 \\
\hline 11:00:00 a.m. & 0 & 1:15:00 p.m. & 7 & 3:15:00 p.m. & 198 \\
\hline 11:05:00 a.m. & 10 & 1:20:00 p.m. & 9 & 3:20:00 p.m. & 156 \\
\hline 11:10:00 a.m. & 7 & 1:25:00 p.m. & 27 & 3:25:00 p.m. & 133 \\
\hline 11:15:00 a.m. & 6 & 1:30:00 p.m. & 0 & 3:30:00 p.m. & 99 \\
\hline 11:20:00 a.m. & 0 & 1:35:00 p.m. & 6 & 3:35:00 p.m. & 181 \\
\hline 11:25:00 a.m. & 0 & 1:40:00 p.m. & 69 & 3:40:00 p.m. & 15 \\
\hline 11:30:00 a.m. & 0 & 1:45:00 p.m. & 0 & 3:45:00 p.m. & 0 \\
\hline 11:35:00 a.m. & 0 & 1:50:00 p.m. & 0 & 3:50:00 p.m. & 0 \\
\hline 11:40:00 a.m. & 10 & 1:55:00 p.m. & 0 & 3:55:00 p.m. & 0 \\
\hline 11:45:00 a.m. & 67 & 2:00:00 p.m. & 43 & 4:00:00 p.m. & 0 \\
\hline 11:50:00 a.m. & 6 & 2:05:00 p.m. & 0 & 4:05:00 p.m. & 0 \\
\hline 11:55:00 a.m. & 10 & 2:10:00 p.m. & 9 & 4:10:00 p.m. & 0 \\
\hline 12:00:00 p.m. & 10 & 2:15:00 p.m. & 0 & 4:15:00 p.m. & 0 \\
\hline 12:05:00 p.m. & 96 & 2:20:00 p.m. & 0 & 4:20:00 p.m. & 0 \\
\hline 12:10:00 p.m. & 0 & 2:25:00 p.m. & 0 & 4:25:00 p.m. & 0 \\
\hline 12:15:00 p.m. & 0 & 2:30:00 p.m. & 142 & 4:30:00 p.m. & 0 \\
\hline 12:20:00 p.m. & 0 & 2:35:00 p.m. & 118 & 4:35:00 p.m. & 49 \\
\hline 12:25:00 p.m. & 0 & 2:40:00 p.m. & 29 & 4:40:00 p.m. & 11 \\
\hline 12:30:00 p.m. & 0 & 2:45:00 p.m. & 147 & 4:45:00 p.m. & 0 \\
\hline 12:35:00 p.m. & 73 & 2:50:00 p.m. & 188 & & \\
\hline 12:40:00 p.m. & 7 & 2:55:00 p.m. & 231 & & \\
\hline 12:45:00 p.m. & 12 & & & & \\
\hline 12:50:00 p.m. & 0 & & & & \\
\hline 12:55:00 p.m. & 39 & & & & \\
\hline \multicolumn{4}{|c|}{ Sedentary time Fitbit ${ }^{\circledR}$ Flex $^{\mathrm{TM}}$ (0 - 24 steps) } & 250 mins & \\
\hline \multicolumn{4}{|c|}{ Sedentary time diary } & 245 mins & \\
\hline \multicolumn{4}{|c|}{ Inaccuracy(0 - 24 steps) } & $+2.0 \%$ & \\
\hline
\end{tabular}


Participant Three. Fitbit ${ }^{\circledast}$ Flex $^{\mathrm{TM}}$ worn between 10.55 AM and 5.05 PM. Green cells denote agreement between 'sitting time' noted in participant diary and zero steps recorded by Fitbit ${ }^{\circledR}$ Flex $^{\mathrm{TM}}$, purple cells denote agreement between 'sitting time' noted in participant diary and $<25$ steps recorded in the same five minute period by the Fitbit ${ }^{\circledast}$ Flex $^{\mathrm{TM}}$. Uncoloured cells indicate agreement between 'active' time noted in participant diary and 25 steps or more recorded in the same five minute period by the Fitbit $^{\circledR}$ Flex $^{\mathrm{TM}}$. Red cells indicate 'active time' noted in the participant diary and $<25$ steps recorded in the same five minute period on the Fitbit $^{\circledR}$ Flex $^{\mathrm{Tm}}$. Blue cells denote 'inactive time noted in the participant diary and 25 steps or more recorded in the same five minute period by the Fitbit $^{\circledR}$ Flex $^{\mathrm{TM}}$.

\begin{tabular}{|c|c|c|c|c|c|}
\hline Time & Steps & Time & Steps & Time & Steps \\
\hline 10:55:00 a.m. & 26 & 1:00:00 p.m. & 589 & 3:00:00 p.m. & 0 \\
\hline 11:00:00 a.m. & 0 & 1:05:00 p.m. & 528 & 3:05:00 p.m. & 0 \\
\hline 11:05:00 a.m. & 29 & 1:10:00 p.m. & 84 & 3:10:00 p.m. & 17 \\
\hline 11:10:00 a.m. & 52 & 1:15:00 p.m. & 426 & 3:15:00 p.m. & 0 \\
\hline 11:15:00 a.m. & 24 & 1:20:00 p.m. & 378 & 3:20:00 p.m. & 213 \\
\hline 11:20:00 a.m. & 181 & 1:25:00 p.m. & 21 & 3:25:00 p.m. & 26 \\
\hline 11:25:00 a.m. & 0 & 1:30:00 p.m. & 28 & 3:30:00 p.m. & 61 \\
\hline 11:30:00 a.m. & 0 & 1:35:00 p.m. & 28 & 3:35:00 p.m. & 206 \\
\hline 11:35:00 a.m. & 0 & 1:40:00 p.m. & 101 & 3:40:00 p.m. & 0 \\
\hline 11:40:00 a.m. & 0 & 1:45:00 p.m. & 0 & 3:45:00 p.m. & 0 \\
\hline 11:45:00 a.m. & 0 & 1:50:00 p.m. & 49 & 3:50:00 p.m. & 0 \\
\hline 11:50:00 a.m. & 113 & 1:55:00 p.m. & 0 & 3:55:00 p.m. & 0 \\
\hline 11:55:00 a.m. & 12 & 2:00:00 p.m. & 7 & 4:00:00 p.m. & 0 \\
\hline 12:00:00 p.m. & 6 & 2:05:00 p.m. & 8 & 4:05:00 p.m. & 0 \\
\hline 12:05:00 p.m. & 44 & 2:10:00 p.m. & 0 & 4:10:00 p.m. & 0 \\
\hline 12:10:00 p.m. & 19 & 2:15:00 p.m. & 7 & 4:15:00 p.m. & 0 \\
\hline 12:15:00 p.m. & 34 & 2:20:00 p.m. & 0 & 4:20:00 p.m. & 0 \\
\hline 12:20:00 p.m. & 39 & 2:25:00 p.m. & 0 & 4:25:00 p.m. & 20 \\
\hline 12:25:00 p.m. & 24 & 2:30:00 p.m. & 0 & 4:30:00 p.m. & 0 \\
\hline 12:30:00 p.m. & 41 & 2:35:00 p.m. & 0 & 4:35:00 p.m. & 0 \\
\hline 12:35:00 p.m. & 15 & 2:40:00 p.m. & 0 & 4:40:00 p.m. & 0 \\
\hline 12:40:00 p.m. & 44 & 2:45:00 p.m. & 0 & 4:45:00 p.m. & 0 \\
\hline 12:45:00 p.m. & 26 & 2:50:00 p.m. & 4 & 4:50:00 p.m. & 93 \\
\hline 12:50:00 p.m. & 30 & 2:55:00 p.m. & 0 & 4:55:00 p.m. & 6 \\
\hline 12:55:00 p.m. & 15 & & & 5:00:00 p.m. & 32 \\
\hline \multicolumn{4}{|c|}{ Sedentary time Fitbit ${ }^{\circledast}$ Flex $^{\mathrm{TM}}$ (0 - 24 steps) } & 235 mins & \\
\hline \multicolumn{4}{|c|}{ Sedentary time diary } & 240 mins & \\
\hline \multicolumn{4}{|c|}{ Inaccuracy (0 - 24 steps) } & $-2.1 \%$ & \\
\hline
\end{tabular}


Participant Four. Fitbit ${ }^{\circledR}$ Flex $^{\mathrm{TM}}$ worn between 9.40 AM and 4.40 PM. Green cells denote agreement between 'sitting time' noted in participant diary and zero steps recorded by Fitbit $^{\circledR} \mathrm{Flex}^{\mathrm{TM}}$, purple cells denote agreement between 'sitting time' noted in participant diary and $<25$ steps recorded in the same five minute period by the Fitbit ${ }^{\circledR} \mathrm{Flex}^{\mathrm{TM}}$. Uncoloured cells indicate agreement between 'active' time noted in participant diary and 25 steps or more recorded in the same five minute period by the Fitbit ${ }^{\circledast}$ Flex $^{\mathrm{TM}}$. Red cells indicate 'active time' noted in the participant diary and $<25$ steps recorded in the same five minute period on the Fitbit $^{\circledR}$ Flex $^{\mathrm{TM}}$. Blue cells denote 'inactive time noted in the participant diary and $\mathbf{2 5}$ steps or more recorded in the same five minute period by the Fitbit $^{\circledR}$ Flex $^{\mathrm{TM}}$.

\begin{tabular}{|c|c|c|c|c|c|}
\hline Time & Steps & Time & Steps & Time & Steps \\
\hline 9:40:00 a.m. & 8 & 12:00:00 p.m. & 94 & 2:25:00 p.m. & 0 \\
\hline 9:45:00 a.m. & 62 & 12:05:00 p.m. & 52 & 2:30:00 p.m. & 0 \\
\hline 9:50:00 a.m. & 0 & 12:10:00 p.m. & 0 & 2:35:00 p.m. & 0 \\
\hline 9:55:00 a.m. & 0 & 12:15:00 p.m. & 0 & 2:40:00 p.m. & 0 \\
\hline 10:00:00 a.m. & 0 & 12:20:00 p.m. & 0 & 2:45:00 p.m. & 6 \\
\hline 10:05:00 a.m. & 53 & 12:25:00 p.m. & 47 & 2:50:00 p.m. & 87 \\
\hline 10:10:00 a.m. & 0 & 12:30:00 p.m. & 0 & 2:55:00 p.m. & 34 \\
\hline 10:15:00 a.m. & 0 & 12:35:00 p.m. & 0 & 3:00:00 p.m. & 0 \\
\hline 10:20:00 a.m. & 0 & 12:40:00 p.m. & 0 & 3:05:00 p.m. & 14 \\
\hline 10:25:00 a.m. & 14 & 12:45:00 p.m. & 0 & 3:10:00 p.m. & 62 \\
\hline 10:30:00 a.m. & 33 & 12:50:00 p.m. & 0 & 3:15:00 p.m. & 40 \\
\hline 10:35:00 a.m. & 62 & 12:55:00 p.m. & 0 & 3:20:00 p.m. & 0 \\
\hline 10:40:00 a.m. & 0 & 1:00:00 p.m. & 78 & 3:25:00 p.m. & 8 \\
\hline 10:45:00 a.m. & 31 & 1:05:00 p.m. & 8 & 3:30:00 p.m. & 0 \\
\hline 10:50:00 a.m. & 68 & 1:10:00 p.m. & 0 & 3:35:00 p.m. & 43 \\
\hline 10:55:00 a.m. & 22 & 1:15:00 p.m. & 13 & 3:40:00 p.m. & 0 \\
\hline 11:00:00 a.m. & 137 & 1:20:00 p.m. & 0 & 3:45:00 p.m. & 0 \\
\hline 11:05:00 a.m. & 25 & 1:25:00 p.m. & 8 & 3:50:00 p.m. & 0 \\
\hline 11:10:00 a.m. & 43 & 1:30:00 p.m. & 7 & 3:55:00 p.m. & 0 \\
\hline 11:15:00 a.m. & 12 & 1:35:00 p.m. & 6 & 4:00:00 p.m. & 44 \\
\hline 11:20:00 a.m. & 6 & 1:40:00 p.m. & 0 & 4:05:00 p.m. & 9 \\
\hline 11:25:00 a.m. & 34 & 1:45:00 p.m. & 0 & 4:10:00 p.m. & 64 \\
\hline 11:30:00 a.m. & 32 & 1:50:00 p.m. & 0 & 4:15:00 p.m. & 28 \\
\hline 11:35:00 a.m. & 13 & 1:55:00 p.m. & 0 & 4:20:00 p.m. & 19 \\
\hline 11:40:00 a.m. & 0 & 2:00:00 p.m. & 0 & 4:25:00 p.m. & 18 \\
\hline 11:45:00 a.m. & 0 & 2:05:00 p.m. & 0 & 4:30:00 p.m. & 63 \\
\hline 11:50:00 a.m. & 6 & 2:10:00 p.m. & 0 & 4:35:00 p.m. & 23 \\
\hline 11:55:00 a.m. & 72 & $\begin{array}{l}\text { 2:15:00 p.m. } \\
\text { 2:20:00 p.m. }\end{array}$ & $\begin{array}{l}0 \\
0\end{array}$ & & \\
\hline \multicolumn{4}{|c|}{ Sedentary time Fitbit ${ }^{\circledR}$ Flex $^{\mathrm{TM}}$ (0 - 24 steps) } & 295 mins & \\
\hline \multicolumn{4}{|c|}{ Sedentary time diary } & 310 mins & \\
\hline \multicolumn{4}{|c|}{ Inaccuracy (0 - 24 steps) } & $-4.8 \%$ & \\
\hline
\end{tabular}


Participant Five. Fitbit ${ }^{\circledR}$ Flex $^{\mathrm{TM}}$ worn between 8.25 AM and 4.45 PM. Green cells denote agreement between 'sitting time' noted in participant diary and zero steps recorded by Fitbit $^{\circledR} \mathrm{Flex}^{\mathrm{TM}}$, purple cells denote agreement between 'sitting time' noted in participant diary and $<25$ steps recorded in the same five minute period by the Fitbit ${ }^{\circledR} \mathrm{Flex}^{\mathrm{TM}}$. Uncoloured cells indicate agreement between 'active' time noted in participant diary and 25 steps or more recorded in the same five minute period by the Fitbit ${ }^{\circledR}$ Flex $^{\mathrm{TM}}$. Red cells indicate 'active time' noted in the participant diary and $<25$ steps recorded in the same five minute period on the Fitbit ${ }^{\oplus}$ Flex $^{\mathrm{TM}}$. Blue cells denote 'inactive time noted in the participant diary and 25 steps or more recorded in the same five minute period by the Fitbit $^{\circledR}$ Flex $^{\mathrm{TM}}$.

\begin{tabular}{|c|c|c|c|c|c|c|c|}
\hline Time & Steps & Time & Steps & Time & Steps & Time & Steps \\
\hline 8:25:00 a.m. & 24 & 10:30:00 a.m. & 0 & 12:35:00 p.m. & 25 & 2:40:00 p.m. & 0 \\
\hline 8:30:00 a.m. & 66 & 10:35:00 a.m. & 0 & 12:40:00 p.m. & 27 & 2:45:00 p.m. & 0 \\
\hline 8:35:00 a.m. & 81 & 10:40:00 a.m. & 19 & 12:45:00 p.m. & 9 & 2:50:00 p.m. & 14 \\
\hline 8:40:00 a.m. & 12 & 10:45:00 a.m. & 73 & 12:50:00 p.m. & 17 & 2:55:00 p.m. & 0 \\
\hline 8:45:00 a.m. & 47 & 10:50:00 a.m. & 12 & 12:55:00 p.m. & 0 & 3:00:00 p.m. & 0 \\
\hline 8:50:00 a.m. & 57 & 10:55:00 a.m. & 51 & 1:00:00 p.m. & 0 & 3:05:00 p.m. & 0 \\
\hline 8:55:00 a.m. & 104 & 11:00:00 a.m. & 50 & 1:05:00 p.m. & 0 & 3:10:00 p.m. & 51 \\
\hline 9:00:00 a.m. & 15 & 11:05:00 a.m. & 7 & 1:10:00 p.m. & 62 & 3:15:00 p.m. & 13 \\
\hline 9:05:00 a.m. & 21 & 11:10:00 a.m. & 45 & 1:15:00 p.m. & 23 & 3:20:00 p.m. & 0 \\
\hline 9:10:00 a.m. & 83 & 11:15:00 a.m. & 20 & 1:20:00 p.m. & 15 & 3:25:00 p.m. & 0 \\
\hline 9:15:00 a.m. & 18 & 11:20:00 a.m. & 0 & 1:25:00 p.m. & 71 & 3:30:00 p.m. & 0 \\
\hline 9:20:00 a.m. & 49 & 11:25:00 a.m. & 0 & 1:30:00 p.m. & 0 & 3:35:00 p.m. & 0 \\
\hline 9:25:00 a.m. & 42 & 11:30:00 a.m. & 11 & 1:35:00 p.m. & 6 & 3:40:00 p.m. & 27 \\
\hline 9:30:00 a.m. & 28 & 11:35:00 a.m. & 33 & 1:40:00 p.m. & 60 & 3:45:00 p.m. & 104 \\
\hline 9:35:00 a.m. & 23 & 11:40:00 a.m. & 36 & 1:45:00 p.m. & 49 & 3:50:00 p.m. & 0 \\
\hline 9:40:00 a.m. & 45 & 11:45:00 a.m. & 43 & 1:50:00 p.m. & 46 & 3:55:00 p.m. & 8 \\
\hline 9:45:00 a.m. & 15 & 11:50:00 a.m. & 21 & 1:55:00 p.m. & 56 & 4:00:00 p.m. & 28 \\
\hline 9:50:00 a.m. & 43 & 11:55:00 a.m. & 49 & 2:00:00 p.m. & 90 & 4:05:00 p.m. & 0 \\
\hline 9:55:00 a.m. & 34 & 12:00:00 p.m. & 21 & 2:05:00 p.m. & 234 & 4:10:00 p.m. & 0 \\
\hline 10:00:00 a.m. & 56 & 12:05:00 p.m. & 68 & 2:10:00 p.m. & 0 & 4:15:00 p.m. & 0 \\
\hline 10:05:00 a.m. & 32 & 12:10:00 p.m. & 30 & 2:15:00 p.m. & 67 & 4:20:00 p.m. & 0 \\
\hline 10:10:00 a.m. & 72 & 12:15:00 p.m. & 18 & 2:20:00 p.m. & 18 & 4:25:00 p.m. & 0 \\
\hline 10:15:00 a.m. & 14 & 12:20:00 p.m. & 38 & 2:25:00 p.m. & 48 & 4:30:00 p.m. & 0 \\
\hline 10:20:00 a.m. & 33 & 12:25:00 p.m. & 66 & 2:30:00 p.m. & 0 & 4:35:00 p.m. & 18 \\
\hline 10:25:00 a.m. & 142 & 12:30:00 p.m. & 90 & 2:35:00 p.m. & 7 & 4:40:00 p.m. & 29 \\
\hline \multicolumn{6}{|c|}{ Sedentary time Fitbit ${ }^{\circledast}$ Flex $^{\mathrm{TM}}$ (0 - 24 steps) } & \multicolumn{2}{|l|}{265 mins } \\
\hline \multicolumn{6}{|c|}{ Sedentary time diary } & \multicolumn{2}{|l|}{ 250mins } \\
\hline \multicolumn{6}{|c|}{ Inaccuracy (0 - 24 steps) } & \multicolumn{2}{|l|}{$+6 \%$} \\
\hline
\end{tabular}


Participant six. Fitbit ${ }^{\circledast}$ Flex $^{\mathrm{TM}}$ worn between 10.05 AM and 4.45 PM. Green cells denote agreement between 'sitting time' noted in participant diary and zero steps recorded by Fitbit $^{\circledR} \mathrm{Flex}^{\mathrm{TM}}$, purple cells denote agreement between 'sitting time' noted in participant diary and $<25$ steps recorded in the same five minute period by the Fitbit ${ }^{\circledR} \mathrm{Flex}^{\mathrm{TM}}$. Uncoloured cells indicate agreement between 'active' time noted in participant diary and 25 steps or more recorded in the same five minute period by the Fitbit ${ }^{\circledR}$ Flex $^{\mathrm{TM}}$. Red cells indicate 'active time' noted in the participant diary and $<25$ steps recorded in the same five minute period on the Fitbit ${ }^{\circledR}$ Flex $^{\mathrm{TM}}$. Blue cells denote 'inactive time noted in the participant diary and $\mathbf{2 5}$ steps or more recorded in the same five minute period by the Fitbit $^{\circledR}$ Flex $^{\mathrm{TM}}$.

\begin{tabular}{|c|c|c|c|c|c|}
\hline Time & Steps & Time & Steps & Time & Steps \\
\hline 10:05:00 a.m. & 224 & 12:20:00 p.m. & 0 & 2:35:00 p.m. & 0 \\
\hline 10:10:00 a.m. & 0 & 12:25:00 p.m. & 4 & 2:40:00 p.m. & 105 \\
\hline 10:15:00 a.m. & 14 & 12:30:00 p.m. & 0 & 2:45:00 p.m. & 19 \\
\hline 10:20:00 a.m. & 0 & 12:35:00 p.m. & 14 & 2:50:00 p.m. & 0 \\
\hline 10:25:00 a.m. & 0 & 12:40:00 p.m. & 0 & 2:55:00 p.m. & 4 \\
\hline 10:30:00 a.m. & 0 & 12:45:00 p.m. & 43 & 3:00:00 p.m. & 14 \\
\hline 10:35:00 a.m. & 26 & 12:50:00 p.m. & 0 & 3:05:00 p.m. & 20 \\
\hline 10:40:00 a.m. & 0 & 12:55:00 p.m. & 0 & 3:10:00 p.m. & 8 \\
\hline 10:45:00 a.m. & 0 & 1:00:00 p.m. & 96 & 3:15:00 p.m. & 24 \\
\hline 10:50:00 a.m. & 23 & 1:05:00 p.m. & 0 & 3:20:00 p.m. & 12 \\
\hline 10:55:00 a.m. & 0 & 1:10:00 p.m. & 0 & 3:25:00 p.m. & 20 \\
\hline 11:00:00 a.m. & 0 & 1:15:00 p.m. & 0 & 3:30:00 p.m. & 0 \\
\hline 11:05:00 a.m. & 0 & 1:20:00 p.m. & 0 & 3:35:00 p.m. & 0 \\
\hline 11:10:00 a.m. & 0 & 1:25:00 p.m. & 0 & 3:40:00 p.m. & 51 \\
\hline 11:15:00 a.m. & 0 & 1:30:00 p.m. & 15 & 3:45:00 p.m. & 4 \\
\hline 11:20:00 a.m. & 60 & 1:35:00 p.m. & 12 & 3:50:00 p.m. & 0 \\
\hline 11:25:00 a.m. & 82 & 1:40:00 p.m. & 0 & 3:55:00 p.m. & 0 \\
\hline 11:30:00 a.m. & 41 & 1:45:00 p.m. & 8 & 4:00:00 p.m. & 0 \\
\hline 11:35:00 a.m. & 117 & 1:50:00 p.m. & 16 & 4:05:00 p.m. & 0 \\
\hline 11:40:00 a.m. & 186 & 1:55:00 p.m. & 6 & 4:10:00 p.m. & 0 \\
\hline 11:45:00 a.m. & 0 & 2:00:00 p.m. & 0 & 4:15:00 p.m. & 0 \\
\hline 11:50:00 a.m. & 0 & 2:05:00 p.m. & 81 & 4:20:00 p.m. & 0 \\
\hline 11:55:00 a.m. & 0 & 2:10:00 p.m. & 109 & 4:25:00 p.m. & 19 \\
\hline 12:00:00 p.m. & 17 & 2:15:00 p.m. & 22 & 4:30:00 p.m. & 98 \\
\hline 12:05:00 p.m. & 0 & 2:20:00 p.m. & 0 & 4:35:00 p.m. & 26 \\
\hline 12:10:00 p.m. & 0 & 2:25:00 p.m. & 0 & 4:40:00 p.m. & 57 \\
\hline 12:15:00 p.m. & 0 & 2:30:00 p.m. & 15 & & \\
\hline \multicolumn{4}{|c|}{ Sedentary time Fitbit ${ }^{\circledR}$ Flex $^{\mathrm{TM}}$ (0 - 24 steps) } & 320 mins & \\
\hline \multicolumn{4}{|c|}{ Sedentary time diary } & 335 mins & \\
\hline \multicolumn{4}{|c|}{ Inaccuracy (0 - 24 steps) } & $-4.4 \%$ & \\
\hline
\end{tabular}


Participant seven. Fitbit ${ }^{\circledR}$ Flex $^{\mathrm{TM}}$ worn between 10.45 AM and 4.55 PM. Green cells denote agreement between 'sitting time' noted in participant diary and zero steps recorded by Fitbit ${ }^{\circledR}$ Flex $^{\mathrm{TM}}$, purple cells denote agreement between 'sitting time' noted in participant diary and $<25$ steps recorded in the same five minute period by the Fitbit ${ }^{\circledast}$ Flex $^{\mathrm{TM}}$. Uncoloured cells indicate agreement between 'active' time noted in participant diary and 25 steps or more recorded in the same five minute period by the Fitbit $^{\circledR}$ Flex $^{\mathrm{TM}}$. Red cells indicate 'active time' noted in the participant diary and $<25$ steps recorded in the same five minute period on the Fitbit $^{\circledR}{ }^{\text {Flex }^{\mathrm{Tm}}}$. Blue cells denote 'inactive time noted in the participant diary and 25 steps or more recorded in the same five minute period by the Fitbit ${ }^{\circledR}$ Flex $^{\mathrm{TM}}$.

\begin{tabular}{|c|c|c|c|c|c|}
\hline Time & Steps & Time & Steps & Time & Steps \\
\hline 10:45:00 a.m. & 0 & 12:55:00 p.m. & 0 & 3:05:00 p.m. & 0 \\
\hline 10:50:00 a.m. & 0 & 1:00:00 p.m. & 143 & 3:10:00 p.m. & 0 \\
\hline 10:55:00 a.m. & 6 & 1:05:00 p.m. & 42 & 3:15:00 p.m. & 0 \\
\hline 11:00:00 a.m. & 0 & 1:10:00 p.m. & 0 & 3:20:00 p.m. & 55 \\
\hline 11:05:00 a.m. & 0 & 1:15:00 p.m. & 44 & 3:25:00 p.m. & 0 \\
\hline 11:10:00 a.m. & 0 & 1:20:00 p.m. & 5 & 3:30:00 p.m. & 0 \\
\hline 11:15:00 a.m. & 0 & 1:25:00 p.m. & 13 & 3:35:00 p.m. & 0 \\
\hline 11:20:00 a.m. & 0 & 1:30:00 p.m. & 158 & 3:40:00 p.m. & 81 \\
\hline 11:25:00 a.m. & 0 & 1:35:00 p.m. & 60 & 3:45:00 p.m. & 4 \\
\hline 11:30:00 a.m. & 0 & 1:40:00 p.m. & 0 & 3:50:00 p.m. & 0 \\
\hline 11:35:00 a.m. & 9 & 1:45:00 p.m. & 0 & 3:55:00 p.m. & 0 \\
\hline 11:40:00 a.m. & 0 & 1:50:00 p.m. & 0 & 4:00:00 p.m. & 14 \\
\hline 11:45:00 a.m. & 42 & 1:55:00 p.m. & 5 & 4:05:00 p.m. & 0 \\
\hline 11:50:00 a.m. & 145 & 2:00:00 p.m. & 0 & 4:10:00 p.m. & 0 \\
\hline 11:55:00 a.m. & 339 & 2:05:00 p.m. & 33 & 4:15:00 p.m. & 0 \\
\hline 12:00:00 p.m. & 164 & 2:10:00 p.m. & 30 & 4:20:00 p.m. & 31 \\
\hline 12:05:00 p.m. & 0 & 2:15:00 p.m. & 17 & 4:25:00 p.m. & 0 \\
\hline 12:10:00 p.m. & 0 & 2:20:00 p.m. & 16 & 4:30:00 p.m. & 0 \\
\hline 12:15:00 p.m. & 0 & 2:25:00 p.m. & 42 & 4:35:00 p.m. & 0 \\
\hline 12:20:00 p.m. & 0 & 2:30:00 p.m. & 0 & 4:40:00 p.m. & 0 \\
\hline 12:25:00 p.m. & 0 & 2:35:00 p.m. & 0 & 4:45:00 p.m. & 0 \\
\hline 12:30:00 p.m. & 0 & 2:40:00 p.m. & 0 & 4:50:00 p.m. & 0 \\
\hline 12:35:00 p.m. & 0 & 2:45:00 p.m. & 122 & & \\
\hline 12:40:00 p.m. & 0 & 2:50:00 p.m. & 23 & & \\
\hline 12:45:00 p.m. & 0 & 2:55:00 p.m. & 0 & & \\
\hline 12:50:00 p.m. & 63 & 3:00:00 p.m. & 0 & & \\
\hline \multicolumn{4}{|c|}{ Sedentary time Fitbit $^{\circledR}$ Flex $^{\mathrm{TM}}$ (0 - 24 steps) } & 285 mins & \\
\hline \multicolumn{4}{|c|}{ Sedentary time diary } & 310 mins & \\
\hline \multicolumn{4}{|c|}{ Inaccuracy (0 - 24 steps) } & $-8.1 \%$ & \\
\hline
\end{tabular}


Participant eight. Fitbit ${ }^{\circledR}$ Flex $^{\mathrm{TM}}$ worn between 8.30 AM and 4.35 PM. Green cells denote agreement between 'sitting time' noted in participant diary and zero steps recorded by Fitbit ${ }^{\circledR}$ Flex $^{\mathrm{TM}}$, purple cells denote agreement between 'sitting time' noted in participant diary and $<25$ steps recorded in the same five minute period by the Fitbit ${ }^{\circledast}$ Flex $^{\mathrm{TM}}$. Uncoloured cells indicate agreement between 'active' time noted in participant diary and 25 steps or more recorded in the same five minute period by the Fitbit $^{\circledR}$ Flex $^{\mathrm{TM}}$. Red cells indicate 'active time' noted in the participant diary and $<25$ steps recorded in the same five minute period on the Fitbit ${ }^{\circledR}$ Flex $^{\mathrm{TM}}$. Blue cells denote 'inactive time noted in the participant diary and 25 steps or more recorded in the same five minute period by the Fitbit ${ }^{\circledR}$ Flex $^{\mathrm{TM}}$.

\begin{tabular}{|c|c|c|c|c|c|c|c|}
\hline Time & Steps & Time & Steps & Time & Steps & Time & Steps \\
\hline 8:30:00 a.m. & 20 & 10:35:00 a.m. & 0 & 12:35:00 p.m. & 259 & 2:35:00 p.m. & 0 \\
\hline 8:35:00 a.m. & 52 & 10:40:00 a.m. & 0 & 12:40:00 p.m. & 390 & 2:40:00 p.m. & 0 \\
\hline 8:40:00 a.m. & 135 & 10:45:00 a.m. & 0 & 12:45:00 p.m. & 54 & 2:45:00 p.m. & 0 \\
\hline 8:45:00 a.m. & 28 & 10:50:00 a.m. & 0 & 12:50:00 p.m. & 176 & 2:50:00 p.m. & 0 \\
\hline 8:50:00 a.m. & 0 & 10:55:00 a.m. & 34 & 12:55:00 p.m. & 16 & 2:55:00 p.m. & 0 \\
\hline 8:55:00 a.m. & 0 & 11:00:00 a.m. & 29 & 1:00:00 p.m. & 0 & 3:00:00 p.m. & 0 \\
\hline 9:00:00 a.m. & 65 & 11:05:00 a.m. & 83 & 1:05:00 p.m. & 0 & 3:05:00 p.m. & 0 \\
\hline 9:05:00 a.m. & 16 & 11:10:00 a.m. & 60 & 1:10:00 p.m. & 0 & 3:10:00 p.m. & 0 \\
\hline 9:10:00 a.m. & 44 & 11:15:00 a.m. & 0 & 1:15:00 p.m. & 0 & 3:15:00 p.m. & 0 \\
\hline 9:15:00 a.m. & 16 & 11:20:00 a.m. & 77 & 1:20:00 p.m. & 50 & 3:20:00 p.m. & 0 \\
\hline 9:20:00 a.m. & 0 & 11:25:00 a.m. & 83 & 1:25:00 p.m. & 4 & 3:25:00 p.m. & 50 \\
\hline 9:25:00 a.m. & 0 & 11:30:00 a.m. & 0 & 1:30:00 p.m. & 102 & 3:30:00 p.m. & 28 \\
\hline 9:30:00 a.m. & 0 & 11:35:00 a.m. & 0 & 1:35:00 p.m. & 0 & 3:35:00 p.m. & 0 \\
\hline 9:35:00 a.m. & 9 & 11:40:00 a.m. & 91 & 1:40:00 p.m. & 86 & 3:40:00 p.m. & 0 \\
\hline 9:40:00 a.m. & 0 & 11:45:00 a.m. & 66 & 1:45:00 p.m. & 0 & 3:45:00 p.m. & 0 \\
\hline 9:45:00 a.m. & 28 & 11:50:00 a.m. & 0 & 1:50:00 p.m. & 0 & 3:50:00 p.m. & 0 \\
\hline 9:50:00 a.m. & 20 & 11:55:00 a.m. & 48 & 1:55:00 p.m. & 84 & 3:55:00 p.m. & 29 \\
\hline 9:55:00 a.m. & 55 & 12:00:00 p.m. & 7 & 2:00:00 p.m. & 0 & 4:00:00 p.m. & 59 \\
\hline 10:00:00 a.m. & 74 & 12:05:00 p.m. & 0 & 2:05:00 p.m. & 0 & 4:05:00 p.m. & 0 \\
\hline 10:05:00 a.m. & 0 & 12:10:00 p.m. & 11 & 2:10:00 p.m. & 47 & 4:10:00 p.m. & 0 \\
\hline 10:10:00 a.m. & 50 & 12:15:00 p.m. & 68 & 2:15:00 p.m. & 30 & 4:15:00 p.m. & 0 \\
\hline 10:15:00 a.m. & 8 & 12:20:00 p.m. & 0 & 2:20:00 p.m. & 62 & 4:20:00 p.m. & 0 \\
\hline 10:20:00 a.m. & 47 & 12:25:00 p.m. & 146 & 2:25:00 p.m. & 15 & 4:25:00 p.m. & 176 \\
\hline 10:25:00 a.m. & 76 & 12:30:00 p.m. & 300 & 2:30:00 p.m. & 73 & 4:30:00 p.m. & 15 \\
\hline 10:30:00 a.m. & 0 & & & & & & \\
\hline \multicolumn{6}{|c|}{ Sedentary time Fitbit ${ }^{\circledR}$ Flex $^{\mathrm{TM}}$ (0 $0-24$ steps) } & 285 mins & \\
\hline \multicolumn{6}{|c|}{ Sedentary time diary } & 300 mins & \\
\hline \multicolumn{6}{|c|}{ Inaccuracy (0 - 24 steps) } & $-5 \%$ & \\
\hline
\end{tabular}


Participant nine. Fitbit ${ }^{\circledR}$ Flex $^{\mathrm{TM}}$ worn between 8.55 AM and 4.45 PM. Green cells denote agreement between 'sitting time' noted in participant diary and zero steps recorded by Fitbit $^{\circledR} \mathrm{Flex}^{\mathrm{TM}}$, purple cells denote agreement between 'sitting time' noted in participant diary and $<25$ steps recorded in the same five minute period by the Fitbit ${ }^{\circledR} \mathrm{Flex}^{\mathrm{TM}}$. Uncoloured cells indicate agreement between 'active' time noted in participant diary and 25 steps or more recorded in the same five minute period by the Fitbit ${ }^{\circledR}$ Flex $^{\mathrm{TM}}$. Red cells indicate 'active time' noted in the participant diary and $<25$ steps recorded in the same five minute period on the Fitbit ${ }^{\circledast}$ Flex $^{\mathrm{TM}}$. Blue cells denote 'inactive time noted in the participant diary and 25 steps or more recorded in the same five minute period by the Fitbit $^{\circledR}$ Flex $^{\mathrm{TM}}$.

\begin{tabular}{|c|c|c|c|c|c|c|c|}
\hline Time & Steps & Time & Steps & Time & Steps & Time & Steps \\
\hline 8:55:00 a.m. & 94 & 10:55:00 a.m. & 0 & 12:50:00 p.m. & 6 & 2:50:00 p.m. & 332 \\
\hline 9:00:00 a.m. & 48 & 11:00:00 a.m. & 5 & 12:55:00 p.m. & 50 & 2:55:00 p.m. & 46 \\
\hline 9:05:00 a.m. & 27 & 11:05:00 a.m. & 0 & 1:00:00 p.m. & 0 & 3:00:00 p.m. & 0 \\
\hline 9:10:00 a.m. & 99 & 11:10:00 a.m. & 0 & 1:05:00 p.m. & 0 & 3:05:00 p.m. & 0 \\
\hline 9:15:00 a.m. & 249 & 11:15:00 a.m. & 36 & 1:10:00 p.m. & 0 & 3:10:00 p.m. & 0 \\
\hline 9:20:00 a.m. & 0 & 11:20:00 a.m. & 144 & 1:15:00 p.m. & 0 & 3:15:00 p.m. & 0 \\
\hline 9:25:00 a.m. & 0 & 11:25:00 a.m. & 14 & 1:20:00 p.m. & 0 & 3:20:00 p.m. & 55 \\
\hline 9:30:00 a.m. & 0 & 11:30:00 a.m. & 0 & 1:25:00 p.m. & 0 & 3:25:00 p.m. & 0 \\
\hline 9:35:00 a.m. & 81 & 11:35:00 a.m. & 0 & 1:30:00 p.m. & 0 & 3:30:00 p.m. & 0 \\
\hline 9:40:00 a.m. & 23 & 11:40:00 a.m. & 0 & 1:35:00 p.m. & 46 & 3:35:00 p.m. & 0 \\
\hline 9:45:00 a.m. & 0 & 11:45:00 a.m. & 0 & 1:40:00 p.m. & 6 & 3:40:00 p.m. & 0 \\
\hline 9:50:00 a.m. & 12 & 11:50:00 a.m. & 7 & 1:45:00 p.m. & 0 & 3:45:00 p.m. & 6 \\
\hline 9:55:00 a.m. & 30 & 11:55:00 a.m. & 51 & 1:50:00 p.m. & 33 & 3:50:00 p.m. & 0 \\
\hline 10:00:00 a.m. & 34 & 12:00:00 p.m. & 5 & 1:55:00 p.m. & 86 & 3:55:00 p.m. & 0 \\
\hline 10:05:00 a.m. & 0 & 12:05:00 p.m. & 0 & 2:00:00 p.m. & 215 & 4:00:00 p.m. & 0 \\
\hline 10:10:00 a.m. & 0 & 12:10:00 p.m. & 0 & 2:05:00 p.m. & 0 & 4:05:00 p.m. & 0 \\
\hline 10:15:00 a.m. & 0 & 12:15:00 p.m. & 0 & 2:10:00 p.m. & 0 & 4:10:00 p.m. & 0 \\
\hline 10:20:00 a.m. & 0 & 12:20:00 p.m. & 100 & 2:15:00 p.m. & 0 & 4:15:00 p.m. & 0 \\
\hline 10:25:00 a.m. & 0 & 12:25:00 p.m. & 30 & 2:20:00 p.m. & 0 & 4:20:00 p.m. & 8 \\
\hline 10:30:00 a.m. & 29 & 12:30:00 p.m. & 9 & 2:25:00 p.m. & 0 & 4:25:00 p.m. & 27 \\
\hline 10:35:00 a.m. & 15 & 12:35:00 p.m. & 420 & 2:30:00 p.m. & 0 & 4:30:00 p.m. & 121 \\
\hline 10:40:00 a.m. & 35 & 12:40:00 p.m. & 273 & 2:35:00 p.m. & 0 & 4:35:00 p.m. & 23 \\
\hline 10:45:00 a.m. & 52 & 12:45:00 p.m. & 338 & 2:40:00 p.m. & 0 & 4:40:00 p.m. & 22 \\
\hline 10:50:00 a.m. & 16 & & & 2:45:00 p.m. & 0 & \multirow{2}{*}{\multicolumn{2}{|c|}{$\begin{array}{l}325 \text { mins } \\
360 \text { mins } \\
-9.7 \%\end{array}$}} \\
\hline \multicolumn{6}{|c|}{$\begin{array}{l}\text { Sedentary time } \text { Fitbit }^{\circledR}{ }^{\mathrm{F}} \text { Flex }^{\mathrm{TM}} \text { (0 - } 24 \text { steps) } \\
\text { Sedentary time diary } \\
\text { Inaccuracy ( } 0 \text { - } 24 \text { steps) }\end{array}$} & & \\
\hline
\end{tabular}


Participant ten. Fitbit ${ }^{\circledR}$ Flex $^{\mathrm{TM}}$ worn between 9.00 AM and 5.00 PM. Green cells denote agreement between 'sitting time' noted in participant diary and zero steps recorded by Fitbit $^{\circledR}$ Flex $^{\mathrm{TM}}$, purple cells denote agreement between 'sitting time' noted in participant diary and $<25$ steps recorded in the same five minute period by the Fitbit ${ }^{\circledR} \mathrm{Flex}^{\mathrm{TM}}$. Uncoloured cells indicate agreement between 'active' time noted in participant diary and 25 steps or more recorded in the same five minute period by the Fitbit ${ }^{\circledR}$ Flex $^{\mathrm{TM}}$. Red cells indicate 'active time' noted in the participant diary and $<25$ steps recorded in the same five minute period on the Fitbit ${ }^{\oplus}$ Flex $^{\mathrm{TM}}$. Blue cells denote 'inactive time noted in the participant diary and $\mathbf{2 5}$ steps or more recorded in the same five minute period by the Fitbit $^{\circledR}$ Flex $^{\mathrm{TM}}$.

\begin{tabular}{|c|c|c|c|c|c|c|c|}
\hline Time & Steps & Time & Steps & Time & Steps & Time & Steps \\
\hline 9:00:00 a.m. & 53 & 11:00:00 a.m. & 0 & 1:00:00 p.m. & 149 & 3:00:00 p.m. & 0 \\
\hline 9:05:00 a.m. & 21 & 11:05:00 a.m. & 0 & 1:05:00 p.m. & 169 & 3:05:00 p.m. & 53 \\
\hline 9:10:00 a.m. & 25 & 11:10:00 a.m. & 0 & 1:10:00 p.m. & 0 & 3:10:00 p.m. & 0 \\
\hline 9:15:00 a.m. & 21 & 11:15:00 a.m. & 0 & 1:15:00 p.m. & 6 & 3:15:00 p.m. & 18 \\
\hline 9:20:00 a.m. & 0 & 11:20:00 a.m. & 70 & 1:20:00 p.m. & 0 & 3:20:00 p.m. & 60 \\
\hline 9:25:00 a.m. & 23 & 11:25:00 a.m. & 233 & 1:25:00 p.m. & 50 & 3:25:00 p.m. & 6 \\
\hline 9:30:00 a.m. & 0 & 11:30:00 a.m. & 74 & 1:30:00 p.m. & 13 & 3:30:00 p.m. & 9 \\
\hline 9:35:00 a.m. & 8 & 11:35:00 a.m. & 0 & 1:35:00 p.m. & 0 & 3:35:00 p.m. & 66 \\
\hline 9:40:00 a.m. & 34 & 11:40:00 a.m. & 5 & 1:40:00 p.m. & 76 & 3:40:00 p.m. & 0 \\
\hline 9:45:00 a.m. & 0 & 11:45:00 a.m. & 4 & 1:45:00 p.m. & 23 & 3:45:00 p.m. & 22 \\
\hline 9:50:00 a.m. & 0 & 11:50:00 a.m. & 0 & 1:50:00 p.m. & 79 & 3:50:00 p.m. & 67 \\
\hline 9:55:00 a.m. & 0 & 11:55:00 a.m. & 199 & 1:55:00 p.m. & 0 & 3:55:00 p.m. & 21 \\
\hline 10:00:00 a.m. & 0 & 12:00:00 p.m. & 196 & 2:00:00 p.m. & 0 & 4:00:00 p.m. & 0 \\
\hline 10:05:00 a.m. & 0 & 12:05:00 p.m. & 0 & 2:05:00 p.m. & 0 & 4:05:00 p.m. & 11 \\
\hline 10:10:00 a.m. & 79 & 12:10:00 p.m. & 0 & 2:10:00 p.m. & 0 & 4:10:00 p.m. & 287 \\
\hline 10:15:00 a.m. & 151 & 12:15:00 p.m. & 0 & 2:15:00 p.m. & 0 & 4:15:00 p.m. & 411 \\
\hline 10:20:00 a.m. & 0 & 12:20:00 p.m. & 0 & 2:20:00 p.m. & 0 & 4:20:00 p.m. & 0 \\
\hline 10:25:00 a.m. & 10 & 12:25:00 p.m. & 0 & 2:25:00 p.m. & 0 & 4:25:00 p.m. & 18 \\
\hline 10:30:00 a.m. & 0 & 12:30:00 p.m. & 0 & 2:30:00 p.m. & 0 & 4:30:00 p.m. & 0 \\
\hline 10:35:00 a.m. & 0 & 12:35:00 p.m. & 0 & 2:35:00 p.m. & 0 & 4:35:00 p.m. & 0 \\
\hline 10:40:00 a.m. & 0 & 12:40:00 p.m. & 0 & 2:40:00 p.m. & 0 & 4:40:00 p.m. & 6 \\
\hline 10:45:00 a.m. & 56 & 12:45:00 p.m. & 107 & 2:45:00 p.m. & 0 & 4:45:00 p.m. & 0 \\
\hline 10:50:00 a.m. & 400 & 12:50:00 p.m. & 7 & 2:50:00 p.m. & 0 & 4:50:00 p.m. & 37 \\
\hline 10:55:00 a.m. & 358 & 12:55:00 p.m. & 44 & 2:55:00 p.m. & 15 & 4:55:00 p.m. & 68 \\
\hline \multicolumn{6}{|c|}{ Sedentary time Fitbit ${ }^{\circledR}$ Flex $^{\mathrm{TM}}$ (0 - 24 steps) } & \multicolumn{2}{|l|}{ 345mins } \\
\hline \multicolumn{6}{|c|}{ Sedentary time diary } & 350 mins & \\
\hline \multicolumn{6}{|c|}{ Inaccuracy (0 - 24 steps) } & \multicolumn{2}{|l|}{$-1.4 \%$} \\
\hline
\end{tabular}


Appendix VIII: Participant Information Sheet: Sedentary occupations: measurement of venous thromboembolism risk factors within the workforce, sedentary time at work and investigation of a possible intervention to promote venous blood flow of the lower limbs. (Dome device at work study) 
Locality: Medical Research Institute of

New Zealand

Lead investigator: Dr Irene Braithwaite
Ethics committee ref.: 15/STH/6

Contact phone number: (04) 8050245

You are invited to take part in a study looking at the amount of time spent seated in an office or callcentre and usage of a novel foot-stool that may improve blood flow in the legs while you are seated for long periods of time. If you don't want to take part you can say no. You don't have to give a reason. If you do want to take part now, but change your mind later, you can pull out of the study at any time.

This Participant Information Sheet tells you why we are doing the study, what is involved, what the benefits and risks to you might be, and what happens at the end of the study. We are very happy to go through this information with you and answer any questions you may have. You do not have to decide today whether or not you do this study. Before you decide you may want to talk about the study with other people, such as family, whānau, friends, or healthcare providers. Feel free to do this.

If you agree to take part in this study, you will be asked to sign the Consent Form on the last page of this document. You will be given a copy of both the Participant Information Sheet and the Consent Form to keep. We can arrange for an interpreter if you need one.

This document is 6 pages long including the Consent Form. Please make sure you have read and understood all the pages.

\section{What is the purpose of the study?}

We want to find out three things.

We want to be able to estimate how much time people spend seated during a working week in their usual office environment and how much they get up and move about.

We want to estimate the proportion of individuals working in a selected office environment who have either had a blood clot or who have one or more known risks for developing blood clots.

We want to see whether it is convenient and practical for office-based workers to use a foot-stool device regularly throughout the working day to improve blood flow through their legs while they are 
sitting down. We know that people sitting down for long periods of time (like when they are flying long distances, or in desk-bound role), may be at increased risk of developing blood clots called deep vein thrombosis (DVT) in their legs. This risk might be partly due to pooling of blood in the leg during long periods of sitting. Using the foot-stool every so often may help reduce pooling by improving the return of blood from the legs when you use it.

To try and get answers to the questions listed above, we are running a trial of approximately 200 office and call-centre-based people using a pedometer (a step-counter) and the foot-stool during a working week.

Understanding how long people sit down for during their usual working day and the proportion of individuals in office-based jobs that have risk factors for blood clots may help in the development of different workplace practices designed to reduce risk. And if we can show that the dome device is convenient, safe and practical, we may be able to introduce it as a simple office tool for people to help reduce the risk of developing blood clots when they are sitting down for long periods of time.

This study is funded by the Medical Research Institute of New Zealand. If you have any questions about the study, you may contact the study investigator:

\author{
Dr Irene Braithwaite \\ Medical Research Institute of New Zealand \\ Ph (04) 8050245 \\ E-mail: irene.braithwaite@mrinz.ac.nz
}

This study has been approved by the New Zealand Health and Disability Ethics Committee: Ref 15/STH/6, date 27 January 2015

\title{
What will my participation in the study involve?
}

If you are aged between 18 and 75 years, you may do this study.

Participation will involve two visits. At the first visit (about 20 minutes long on a Monday morning), a researcher will come and see you at your place of work to complete an informed consent, and to do a brief questionnaire about risk factors for blood clots and how long you sit for during the day. They will then show you how to use the pedometer and the foot-stool. 
The study researcher will give you a diary, in which you will be asked to record your daily usage of the foot-stool. You will also be asked to write down any issues or problems you had when using the foot-stool.

The researcher will visit you again on the following Monday morning to collect the pedometer, the foot-stool, to review your diary with you and ask you about whether you would use the foot-stool again. This visit should take only a few minutes.

\section{What are the possible benefits and risks of this study?}

You may find the use of the foot-stool slightly awkward initially, but this should not be painful. If you experience any pain or significant discomfort/distress, you may withdraw from the trial. If you do stop using the foot-stool we will ask you to write the reason for doing so in your diary and we will still collect your diary, the foot-stool and the pedometer from you on the following Monday morning.

Leaving the foot stool in the open walk areas of your office may create a trip hazard; we recommend that you keep it under your desk and out of the way of common walking areas as much as possible.

There are no other anticipated risks from participating in this study.

\section{Who pays for the study?}

We do not anticipate that you will incur any costs associated with taking part in this study.

\section{What if something goes wrong?}

In the event you were injured in this study, which is unlikely, you may be eligible for compensation from ACC. You will have to lodge a claim with ACC, which may take some time to assess. If your claim is accepted, you may receive funding to assist in your recovery.

If you have private health or life insurance, you may wish to check with your insurer that taking part in this study won't affect your cover.

\section{What are my rights?}

Your participation in this study is voluntary. You can choose not to participate at all, or withdraw at any time even after you have given your consent and started the study.

You have the ability to see any information collected about you during the study, and if you request, a researcher will be available to answer any questions you may have relating to that information. 
All information collected about you will be stored confidentially in secure premises. You will not be able to be identified individually in any published study results.

\section{What happens after the study or if I change my mind?}

The foot-stool being tested is not commercially available and the prototypes will have to be collected after the study has ended. The pedometers will also be collected form you after the study has ended.

Data gathered during this study, including any of your own personal information will be securely stored for 15 years by the MRINZ to meet our ethical and Good Clinical Practice responsibilities. After this period of time, the records will be destroyed to maintain confidentiality and privacy of all participants.

It is possible we may use some of the data from this study to contribute to future studies. If we do so, the data will be used in such a way that you will not be personally identified.

During the informed consent process, you will have the opportunity to tell us if you would like a copy of the final results of this study. We should be able to provide the results within approximately 6 months of study completion. The MRINZ aims to have all studies published in reputable peerreviewed journals, and to present our findings in medical and scientific forums. You will not be able to be personally identified in any of these publications or presentations.

\section{Who do I contact for more information or if I have concerns?}

If you have any questions, concerns or complaints about the study at any stage, you can contact:

Dr Irene Braithwaite

Senior Research Fellow

Medical Research Institute of New Zealand

(04) 8050245

Irene.braithwaite@mrinz.ac.nz

If you want to talk to someone who isn't involved with the study, you can contact an independent health and disability advocate on: 
Phone: 0800555050

Fax:

08002 SUPPORT (0800 2787 7678)

Email: $\quad$ advocacy@hdc.org.nz

For Maori Health support please contact :

Whānau Care Services, Cultural Care Centre, Level 2, Wellington Hospital

Phone: $\quad 0800999442$ or 048060948

Email: $\quad \underline{\text { wcs@ccdhb.org.nz }}$ 


\section{Consent Form}

\section{If you need an INTERPRETER, please tell us.}

\section{All of the 'Yes' boxes must be ticked to be able to progress on to the study.}

\begin{tabular}{l}
\hline I have read, or have had read to me in my first language, and I understand the Participant \\
Information Sheet. \\
\hline I have been given sufficient time to consider whether or not to participate in this study. \\
\hline I have had the opportunity to use a legal representative, whanau/ family support or a \\
friend to help me ask questions and understand the study. \\
$\begin{array}{l}\text { I am satisfied with the answers I have been given regarding the study and I have a copy of } \\
\text { this consent form and information sheet. }\end{array}$ \\
$\begin{array}{l}\text { I understand that taking part in this study is voluntary (my choice) and that I may } \\
\text { withdraw from the study at any time without this affecting my medical care. }\end{array}$ \\
$\begin{array}{l}\text { I consent to the research staff collecting and processing my information, including } \\
\text { information about my health. }\end{array}$ \\
$\begin{array}{l}\text { If I decide to withdraw from the study, I agree that the information collected about me up } \\
\text { to the point when I withdraw may continue to be processed. }\end{array}$ \\
$\begin{array}{l}\text { I consent to the study researcher contacting my GP in the event of any significant medical } \\
\text { problems during the study. }\end{array}$
\end{tabular}

I agree to an approved auditor appointed by the New Zealand Health and Disability Ethic Committees, or any relevant regulatory authority or their approved representative reviewing my relevant medical records for the sole purpose of checking the accuracy of the information recorded for the study.

I understand that my participation in this study is confidential and that no material, which could identify me personally, will be used in any reports on this study.

I understand the compensation provisions in case of injury during the study. Yes $\square$

I know who to contact if I have any questions about the study in general. Yes $\square$

I understand my responsibilities as a study participant.

Yes $\square$

Do you wish to receive a summary of the results from the study? Yes / No 


\section{Declaration by participant:}

I hereby consent to take part in this study.

Participant's name:

Signature:

Date:

\section{Declaration by member of research team:}

I have given a verbal explanation of the research project to the participant, and have answered the participant's questions about it.

I believe that the participant understands the study and has given informed consent to participate.

Researcher's name:

Signature:

Date: 
Appendix IX: Questionnaire: Sedentary occupations: measurement of venous thromboembolism risk factors within the workforce, sedentary time at work and investigation of a possible intervention to promote venous blood flow of the lower limbs. (Dome device at work study). 


\section{Section I: Demographic detalls}

Name:

Ethnicity

Date Of Birth:

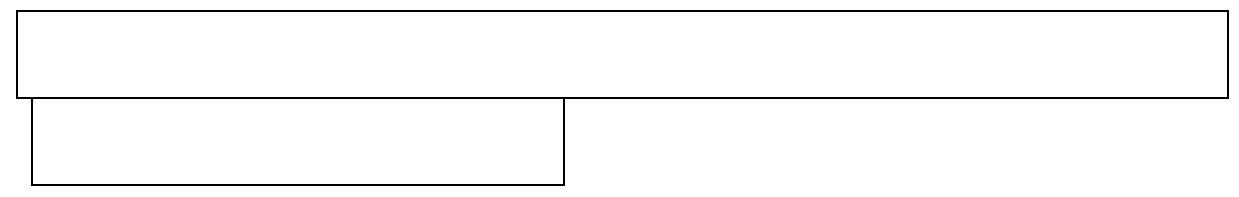

Gender:

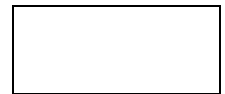

Weight:

Height:

Role title:

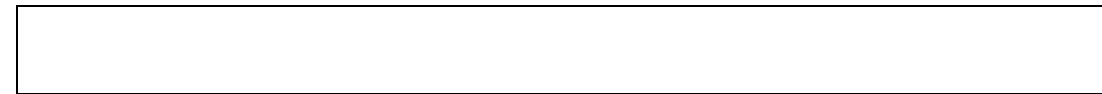

Brief description of role:

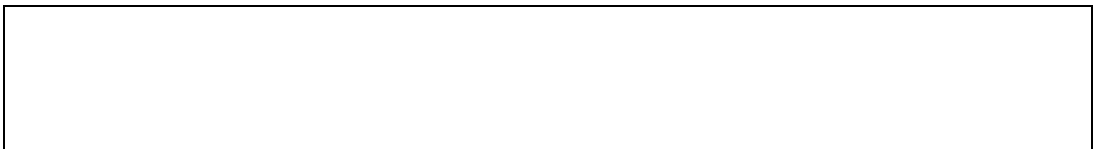

\section{Section II: VTE risk factors}

Have you had a Blood Clot previously?

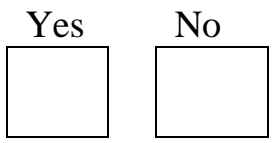

Previous history of Blood Clot - specify:

1.

2.

3.

Family history of Blood Clot - specify:

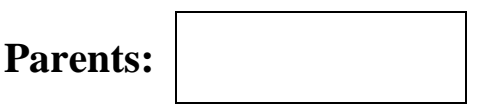

Siblings:

Other: 


\section{Part A: Do you have any of the following blood disorders?}

Antithrombin III deficiency

Protein C deficiency

Protein S deficiency

Factor V Leiden mutation (heterozygous)

Factor V Leiden mutation (homozygous)

Prothrombin gene mutation

Antiphospholipid antibodies

\begin{tabular}{|l|l|}
\multicolumn{1}{l|}{ Yes } & No \\
\hline & \\
\hline & \\
\hline & \\
\hline & \\
\hline & \\
\hline & \\
\hline & \\
\hline
\end{tabular}

Details:

Part B: In the last 4 weeks have you undertaken any of the following?

Long distance travel $(\geq 4 \mathrm{hrs})$ :

Car

International air travel

Other (specify)
Date
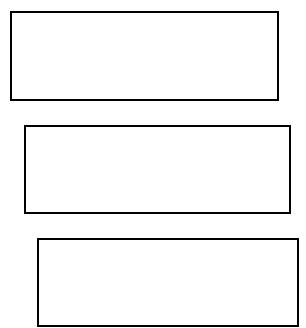

Duration of travel Max. hours per sector Total Duration (hrs)
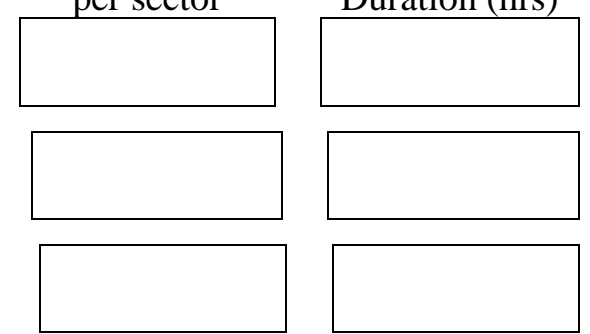


\section{Part C: In the last 4 weeks have you had any of the following?}

Surgery

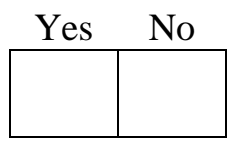

Details

Other immobility (e.g.Hospital admission, drug overdose, , wheelchair bound, bedrest) $\geq 2$ days

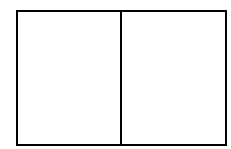

Cast/plaster and/or traumatic injury

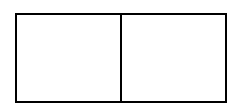

Pregnancy or hormonal treatment including oral contraceptive pill

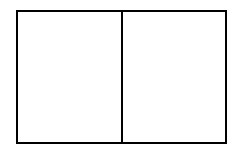

Malignancy or chronic disease

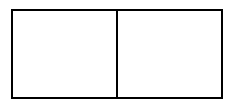

Smoking

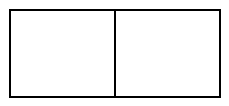

History of varicose veins

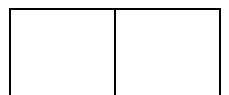

Part D: In the last 4 weeks have you done any of the following?

Strenuous exercise ( $\geq 30$ mins): $\quad$ Details of the form of exercise

Specify whether road or MTB,

Cycling

Rowing / kayaking / waka ama

Weights (i.e. squats, lunges)

Long distance running

Other strenuous exercise
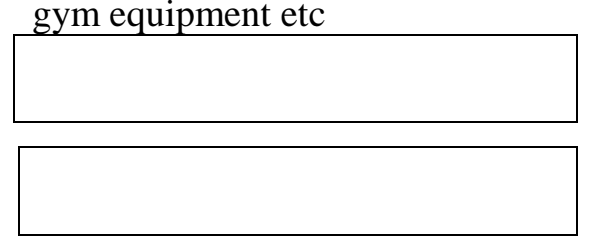

Frequency and duration of exercise

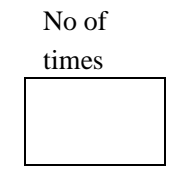

Longest

duration (hrs)
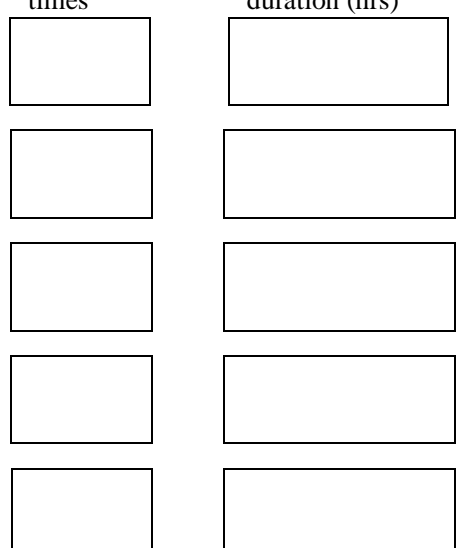

Note: "strenuous exercise" is defined as: cardiovascular or weight related exercise that involves repetitive physical movements 


\section{Section III: Medications}

In the last 4 weeks have you taken any of the following medications?

Anti-thrombotic agents

Enoxaparin / clexane

Warfarin

Aspirin

Clopidogrel

Dipyridamole

Other

Pro-thrombotic agents

Anti-cancer medications

Hormone Replacement Therapy

Oral Contraceptive Pill

Tranexamic acid

Other

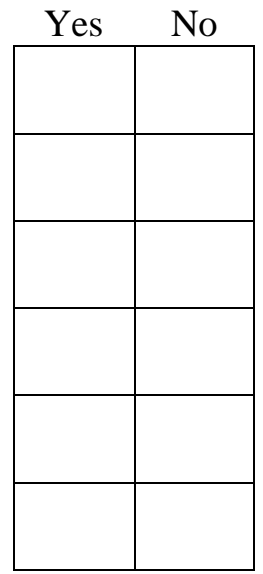

Yes No

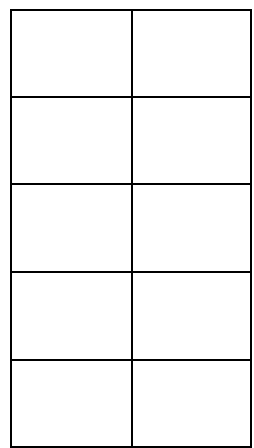

Details:

Details of treatment i.e. tamoxifen, anastrozole, chemotherapy etc

All other medications: (list) 


\section{Section IV: Sitting habits assoclated with work}

\section{In the last 4 weeks at work:}

1. How many days a week did you work?

2. How many hours did you spend at work in a USUAL working day?

3. What was MAXIMUM number of hours you spent at work in one day?

4. What was the total amount of time you USUALLY spent sitting in any one day?

5. What was the MAXIMUM total amount of time you spent sitting in any one day?

6. What was the USUAL amount of time you spent sitting without getting up?

7. What was the MAXIMUM amount of time you spent sitting without getting up?

8. No of days with $\geq 10$ hours sitting at work:

\section{In the last 4 weeks at home:}

1. Did you sit for any work related activities at home?

2. What was the total amount of time you USUALLY spent sitting for work related activities each day?

3. What was MAXIMUM amount of time you sat for work related activities in any one day?

4. What was the USUAL amount of time you spent sitting for work related activities without getting up?

5. What was the MAXIMUM amount of time you spent sitting for work related activities without getting up?

6. No of days with $\geq 10$ hours sitting at work OR at home for work-related activities: 


\section{Section V: Office structure}

1. Do you have a specific desk and chair at work?

2. Is your chair adjustable?

3. Can you stretch out your legs fully while seated?

4. Do you work in an office environment for more than $50 \%$ of your working time?

5. Do you eat lunch at your work desk?

Always:

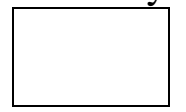

Usually:

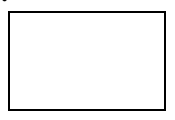

Occasionally:
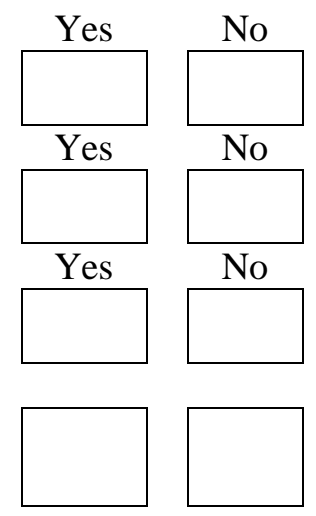

Never:

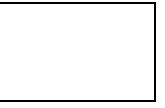

6. Do you have a computer reminder on your work computer, to get up periodically from being seated? (select only one option)

(a) I don't have a specific work computer

(b) I have a specific work computer but am not aware of having a computer reminder installed on it

(c) My work computer which has a computer reminder, but I tend to ignore it, or it has been turned off

(d) I have a computer reminder and I usually get up when reminded to do so

If yes: how frequently does the reminder prompt you to get up? mins

6. Do you stand up for prolonged periods without moving around? (i.e. more than $1 \mathrm{hr})$

7. Do you stand, on average, for more than 6 hours/day at work?
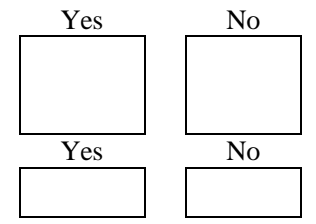

\section{If yes please specify:}

\section{Investigators signature:}

Date: 
Appendix X: Participant Diary: Sedentary occupations: measurement of venous thromboembolism risk factors within the workforce, sedentary time at work and investigation of a possible intervention to promote venous blood flow of the lower limbs. (Dome device at work study). 


\section{Dome Device at Work study - Weekly Diary}

\section{Study ID:}

\section{Date study initiated:}

\section{Place of work: $\quad$ Office / Call-centre (circle appropriate answer)}

Please try and use the Dome device at least once every hour for a minimum of one minute at a time, and fill out the diary on page 3 as per the example below. Please write in this diary the times you started and finished work, the time each day that you used the Dome foot-stool, and how long you used it for in seconds.

\begin{tabular}{|c|l|l|l|l|l|}
\hline Day of week & \multicolumn{1}{|c|}{ Monday } & \multicolumn{1}{|c|}{ Tuesday } & \multicolumn{1}{c|}{ Wednesday } & \multicolumn{1}{c|}{ Thursday } & Friday \\
\hline $\begin{array}{c}\text { TIME WORK } \\
\text { STARTED }\end{array}$ & \multicolumn{1}{|c|}{$08: 15$} & $08: 00$ & $07: 55$ & $08: 25$ & $08: 15$ \\
\hline $\begin{array}{c}\text { TIME WORK } \\
\text { FINISHED }\end{array}$ & $16: 15$ & $16: 45$ & $16: 00$ & $16: 45$ & $16: 50$ \\
\hline Time used (24 hr) & 10.10 & 08.45 & 20.00 & 20.30 & 21.00 \\
\hline Duration (secs) & 60 & 50 & 60 & 45 & 45 \\
\hline Time used & 11.25 & 10.00 & 09.15 & 22.00 & 22.00 \\
\hline Duration (secs) & 35 & 70 & 65 & 30 & 60 \\
\hline
\end{tabular}

Investigators will complete a brief final questionnaire with you when they come to pick up your diary, pedometer and foot-stool at the end of the study.

This diary will be reviewed with you by the study researcher on:

Day:

Date:

Time (approximately) 
Please note down any POSITIVE comments you have about the Dome Device

Please note down any NEGATIVE comments you have about the Dome Device 


\begin{tabular}{|c|c|c|c|c|c|c|c|}
\hline $\begin{array}{l}\text { TIME WORK } \\
\text { STARTED } \\
\text { TIIE WORK } \\
\text { FINISHED }\end{array}$ & Monday & Tuesday & Wednesday & Thursday & Friday & Saturday & Sunday \\
\hline \multicolumn{8}{|c|}{ FOOT-STOOL USE } \\
\hline \multicolumn{8}{|l|}{ Time used } \\
\hline \multicolumn{8}{|l|}{ Duration (secs) } \\
\hline \multicolumn{8}{|l|}{ Time used } \\
\hline \multicolumn{8}{|l|}{ Duration (secs) } \\
\hline \multicolumn{8}{|l|}{ Time used } \\
\hline \multicolumn{8}{|l|}{ Duration (secs) } \\
\hline \multicolumn{8}{|l|}{ Time used } \\
\hline \multicolumn{8}{|l|}{ Duration (secs) } \\
\hline \multicolumn{8}{|l|}{ Time used } \\
\hline \multicolumn{8}{|l|}{ Duration (secs) } \\
\hline \multicolumn{8}{|l|}{ Time used } \\
\hline \multicolumn{8}{|l|}{ Duration (secs) } \\
\hline \multicolumn{8}{|l|}{ Time used } \\
\hline \multicolumn{8}{|l|}{ Duration (secs) } \\
\hline \multicolumn{8}{|l|}{ Time used } \\
\hline Duration (secs) & & & & & & & \\
\hline
\end{tabular}




\begin{tabular}{|c|c|c|c|c|c|c|c|}
\hline Page 2 & Monday & Tuesday & Wednesday & Thursday & Friday & Saturday & Sunday \\
\hline \multicolumn{8}{|l|}{ Time used } \\
\hline \multicolumn{8}{|l|}{ Duration (secs) } \\
\hline \multicolumn{8}{|l|}{ Time used } \\
\hline \multicolumn{8}{|l|}{ Duration (secs) } \\
\hline \multicolumn{8}{|l|}{ Time used } \\
\hline \multicolumn{8}{|l|}{ Duration (secs) } \\
\hline \multicolumn{8}{|l|}{ Time used } \\
\hline \multicolumn{8}{|l|}{ Duration (secs) } \\
\hline \multicolumn{8}{|l|}{ Time used } \\
\hline \multicolumn{8}{|l|}{ Duration (secs) } \\
\hline \multicolumn{8}{|l|}{ Time used } \\
\hline \multicolumn{8}{|l|}{ Duration (secs) } \\
\hline \multicolumn{8}{|l|}{ Time used } \\
\hline \multicolumn{8}{|l|}{ Duration (secs) } \\
\hline \multicolumn{8}{|l|}{ Time used } \\
\hline \multicolumn{8}{|l|}{ Duration (secs) } \\
\hline \multicolumn{8}{|l|}{ Time used } \\
\hline Duration (secs) & & & & & & & \\
\hline
\end{tabular}




\section{Dome Device at Work study - End of study questionnaire}

\section{How convenient was it to use the device while you were at work?}
1. Very convenient
2. Quite convenient
3. Slightly inconvenient
4. Very inconvenient

How easy was the device to use?
1. Very easy
2. Quite easy
3. Not that easy
4. Very hard

\section{How comfortable was the device to use?}

1. Very comfortable

2. Quite comfortable

3. Slightly uncomfortable

4. Very uncomfortable

How likely would you be to use this device reqularly in the future (ie at least hourly on a daily basis)?
1. Very likely
2. Quite likely
3. Not that likely
4. Very unlikely

Did you find the device easier to use with or without shoes?
1. With shoes
2. Without shoes
3. Didn't make a difference

If you wore shoes, what type were they (eg running shoes, sneakers, sandals, heels)

\section{Participant Number:}

Date:

\section{How likely would you be to use this device on a long haul plane flight?}
1. Very likely
2. Quite likely
3. Not that likely
4. Very unlikely 
Appendix XI: Participant Information Sheet: Venous thromboembolism prevention in lower leg injury requiring immobilisation: a feasibility study and open-label trial of Jet Impulse Technology (JIT) within-cast. (JIT in-cast study). 
Locality: Medical Research Institute of New Zealand

Lead investigator: Dr Irene Braithwaite
Ethics committee ref: 14/STH/138

Contact phone number: (04) 8050245

You are invited to take part in a study assessing the effectiveness of a device designed to improve blood flow in a leg after it has been placed in a below-knee plaster cast. Whether or not you take part is your choice. If you don't want to take part, you don't have to give a reason, and it won't affect the care you receive. If you do want to take part now, but change your mind later, you can pull out of the study at any time.

This Participant Information Sheet will help you decide if you'd like to take part. It sets out why we are doing the study, what your participation would involve, what the benefits and risks to you might be, and what would happen after the study ends. We will go through this information with you and answer any questions you may have. Because your leg needs to be casted today, you will need to decide before it is casted whether you wish to take part in this study. You do not have to decide today whether or not you will participate in this study. Before you decide you may want to talk about the study with other people, such as family, whānau, friends, or healthcare providers. Feel free to do this.

If you agree to take part in this study, you will be asked to sign the Consent Form on the last page of this document. You will be given a copy of both the Participant Information Sheet and the Consent Form to keep. We can arrange for an interpreter if you need one.

This document is 7 pages long, including the Consent Form. Please make sure you have read and understood all the pages.

\section{What is the purpose of the study?}

We know that when people have casts on their legs they may be at increased risk of developing blood clots called deep vein thrombosis (DVT). This is because the cast prevents you from using your usual muscles of the foot, ankle and calf to promote blood flow. We want to see whether it is possible to use a small pump-like device called Jet Impulse Technology (JIT) around your foot, while your leg is in a cast, to improve blood flow in your leg. Using the JIT system may help reduce the risk of DVT by improving the return of blood from the legs. We want 70 people to try out this device 
while their leg is in a cast, up to a maximum of 8 weeks. We want to find out how much they use the device, and what sort of problems (if any), they might have while using it. If this looks like a viable option, we would consider undertaking a larger trial to compare the effectiveness of this device to oral medications that are also used to reduce the risk of DVT

This study is funded by the Health Research Council of New Zealand and is being undertaken by the Medical Research Institute of New Zealand. If you have any questions about the study, you may contact the study investigator:

Dr Irene Braithwaite

Medical Research Institute of New Zealand

$\mathrm{Ph}$ (04) 8050245

E-mail: irene.braithwaite@mrinz.ac.nz

This study has been approved by the New Zealand Health and Disability Ethics Committee: 14/STH/138

\section{What will my participation in the study involve?}

If you are aged between 18 and 70 years, and have no current or previous history of blood vessel or muscular problems in your legs, and are not already taking medication to thin your blood, you may be eligible for this study.

Participation will involve having a small inflatable foot-pad placed around the sole of your foot before your leg cast is put on. A portable pump will be attached to the foot-pad via an air hose, and it will blow up the part of the foot-pad around your foot. It will take less than a second to blow up, stay inflated for 3 seconds, and then slowly deflate. This will happen every minute. The maximum pressure the device can blow up to is $130 \mathrm{mmHg}$ ( $\mathrm{mm}$ of mercury). This is about the same as a blood pressure cuff on your arm when a health professional takes your blood pressure.

We want you to have the JIT system working as much as possible while you are immobile (e.g. on the couch, at the computer, even in bed at night). It is most effective when it is used as much as possible. You do not need to use it while you are moving around on your crutches, going to the bathroom, showering, or outdoors. 
The first visit will take place today, as part of your first presentation to fracture clinic with your injury. If you agree to participate in the study, the fracture clinic staff and research staff will work together to undertake an informed consent, take a brief medical history, and examine your legs to make sure there are no problems that will exclude you from the study. Then we will also show you how to use the JIT system, ensure you are able to participate in the study, and the fracture clinic staff will cast your leg with the JIT system inside it.

The research staff will come and see you each time you come in for a fracture clinic appointment. As well as seeing the orthopaedic doctor as you normally would, the researchers and fracture clinic staff will

Make sure the JIT system is working properly

Ask you questions about how and when you are using the device

Remove the cast and the old foot-pad from inside the cast

Examine your leg for evidence of a DVT and to make sure the skin of your leg and foot is okay.

If there are no issues and you are happy to carry on, researchers and fracture clinic staff will place a new foot-pad to the JIT system and replace the cast

We expect these follow up visits for cast and foot-pad changes would be at 2 weeks, 4 weeks and 6 weeks. At 8 weeks, even if you need another cast, the footpad will be removed and the pump unit will be returned to the researchers.

We also want to check the veins in your leg to see if there are any blood clots while you are on the study. So we will organize for you to attend Pacific Radiology offices at 2 weeks, 4 weeks and 8 weeks to have an ultrasound of the veins in your casted leg. An ultrasound is like the tests that pregnant women have. A gel is applied to the leg and a device (probe) is used to measure the blood flow in the veins of your leg. At week 2 and week 4, you are likely to still have your leg in a cast, so the ultrasound will be done of the veins in your thigh. At week 8 , it is most likely that your cast or moonboot will have been removed, and an ultrasound will be done of your entire leg. If we find evidence of a blood clot, you will be advised, and we will start treatment if it is indicated.

The orthopaedic doctors may decide you don't need a leg cast at any of your clinic visits. If this occurs you will not need the JIT system anymore and the researchers will take it back to allocate to another participant. 
If you develop any problems with your cast between your follow up visits you may call the orthopedic clinic to arrange a review sooner than your planned appointment. If you develop symptoms of a blood clot in your leg (pain - like a heavy ache in the affected area, warmth of your leg, and redness behind the knee), please call the researchers if it is within usual working hours and they will organize for you to have an assessment and investigations as required. If it is outside of usual working hours, you will need to attend the After Hours Medical Centre or your local Emergency Department for further evaluation If you develop symptoms of blood clots in your lungs (breathlessness, chest pain, a mild fever, coughing up blood, fainting), then you should seek emergency treatment - call an ambulance. If you develop a blood clot in the course of this study that requires treatment, you will be treated in accordance with Wellington District Health Board practice and you will be reviewed at an outpatient clinic for Venous Thromboembolism.

The study will be completed with a final follow up call 6 weeks after:

- You decide you don't want to use the JIT system any more

- You are withdrawn from the study for any reason

- The orthopedic doctors decide it's time for you to stop wearing a leg cast OR at 8 weeks, whichever comes first

We believe that participating in this study may mean that your fracture clinic appointment may take about half an hour longer than usual. We will do our best to minimize any delays.

If you wish to have family / whanau present while you attend any appointments, feel free to do this.

\section{What are the possible benefits and risks of this study?}

The JIT system: This is a study designed to find out how people will use the device, so any information about use (or disuse) is valuable.

- You may find the JIT system slightly disconcerting initially, but it should not be painful. It is possible to have the device set to a lower pressure setting if you have sensitive feet.

- You may find it too intrusive to wear at night, but give it a go. You can always turn it off if it keeps you awake. 
- It is possible that there may be some damage to the skin as a result of wearing the foot pump device inside the cast and using it for up to 2 weeks at a time. We recommend that you keep your cast as dry as possible to reduce any issues with dampness causing chafing to your skin (this is usual practice for all casts anyway). We will inspect your feet and lower leg carefully at each visit to see if there are any problems with your skin. If you develop any problems between appointments, you can call the study investigator to organize a review sooner. As this kind of study has not been done before, any information about problems associated with long term use of the JIT system inside a cast will be very valuable information.

- If you experience any pain or significant discomfort/distress, or if the researchers are concerned about your wellbeing during the trial, you may be withdrawn from the trial.

In order to measure the blood flow in your legs using ultrasound equipment it is necessary to apply a viscous gel to the skin for the duration of each measurement. In some individuals with sensitive skin there is a small possibility of minor skin irritation from the use of this gel.

There are no other anticipated risks from participating in this study.

\section{Who pays for the study?}

We do not anticipate that you will incur any costs associated with taking part in this study. We will provide reimbursement of $\$ 50$ for each follow-up visit to cover some of the costs or inconvenience that may have been incurred as a result of you participating.

\section{What if something goes wrong?}

If you were injured in this study, which is unlikely, you would be eligible for compensation from ACC just as you would be if you were injured in an accident at work or at home. You will have to lodge a claim with ACC, which may take some time to assess. If your claim is accepted, you will receive funding to assist in your recovery.

If you have private health or life insurance, you may wish to check with your insurer that taking part in this study won't affect your cover. 


\section{What are my rights?}

You have the ability to see any information collected about you during the study, and if you request, a researcher will be available to provide explanation, or to answer any questions you may have relating to that information.

All information collected about you will be stored confidentially in secure offices. You will not be able to be identified individually in any published study results.

\section{What happens if I change my mind and withdraw from the study?}

Your participation in this study is voluntary. You can choose not to participate at all, or withdraw at any time even after you have given your consent and started the study. Choosing not to participate, or choosing to withdraw after you have started the study will not affect your future healthcare.

If you decide to withdraw from the study, you may do so at any time, you just need to let me know at the time. Any information collected about you up to the point when you withdraw may continue to be used in the study.

\section{What happens after the study?}

Data gathered during this study, including any of your own personal information will be securely stored for 15 years by the MRINZ to meet our ethical and Good Clinical Practice responsibilities. After this period of time, the records will be destroyed to maintain confidentiality and privacy of all participants.

It is possible we may use some of the data from this study to contribute to future studies. This would comprise ultrasound measurement results and other non-identifiable data.

You have the opportunity to tell us if you would like a copy of the final results of this study during the informed consent process. This should be able to be done within approximately 6 months of study completion. The MRINZ aims to have all studies written up and published in reputable journals, and to present our findings in medical and scientific forums. You will not be able to be personally identified in any of these publications or presentations.

\section{Who do I contact for more information or if I have concerns?}

If you have any questions, concerns or complaints about the study at any stage, you can contact: 
Dr Irene Braithwaite

Senior Research Fellow

Medical Research Institute of New Zealand

(04) 8050245

Irene.braithwaite@mrinz.ac.nz

If you want to talk to someone who isn't involved with the study, you can contact an independent health and disability advocate on:

Phone: 0800555050

Fax:

08002 SUPPORT (0800 2787 7678)

Email: advocacy@hdc.org.nz

For Maori health support please contact :

Whānau Care Services, Cultural Care Centre, Level 2, Wellington Hospital

Phone: $\quad 0800999442$ or 048060948

Email: $\quad$ wcs@ccdhb.org.nz 


\section{Consent Form}

\section{If you need an INTERPRETER, please tell us.}

\begin{tabular}{|c|c|}
\hline $\begin{array}{l}\text { I have read, or have had read to me in my first language, and I understand the Participant } \\
\text { Information Sheet. }\end{array}$ & Yes $\square$ \\
\hline I have been given sufficient time to consider whether or not to participate in this study. & Yes $\square$ \\
\hline $\begin{array}{l}\text { I have had the opportunity to use a legal representative, whanau/ family support or a } \\
\text { friend to help me ask questions and understand the study. }\end{array}$ & Yes $\square$ \\
\hline $\begin{array}{l}\text { I am satisfied with the answers I have been given regarding the study and I have a copy of } \\
\text { this consent form and information sheet. }\end{array}$ & Yes $\square$ \\
\hline $\begin{array}{l}\text { I understand that taking part in this study is voluntary (my choice) and that I may } \\
\text { withdraw from the study at any time without this affecting my medical care. }\end{array}$ & Yes $\square$ \\
\hline $\begin{array}{l}\text { I consent to the research staff collecting and processing my information, including } \\
\text { information about my health. }\end{array}$ & Yes $\square$ \\
\hline $\begin{array}{l}\text { If I decide to withdraw from the study, I agree that the information collected about me up } \\
\text { to the point when I withdraw may continue to be processed. }\end{array}$ & Yes $\square$ \\
\hline $\begin{array}{l}\text { I wish to have my GP or current provider informed about my participation in the study } \\
\text { and of any significant abnormal results obtained during the study (please circle). }\end{array}$ & Yes / No \\
\hline $\begin{array}{l}\text { I agree to an approved auditor appointed by the New Zealand Health and Disability Ethic } \\
\text { Committees, or any relevant regulatory authority or their approved representative } \\
\text { reviewing my relevant medical records for the sole purpose of checking the accuracy of } \\
\text { the information recorded for the study. }\end{array}$ & Yes $\square$ \\
\hline $\begin{array}{l}\text { I understand that my participation in this study is confidential and that no material, which } \\
\text { could identify me personally, will be used in any reports on this study. }\end{array}$ & Yes $\square$ \\
\hline I understand the compensation provisions in case of injury during the study. & Yes $\square$ \\
\hline I know who to contact if I have any questions about the study in general. & Yes $\square$ \\
\hline I understand my responsibilities as a study participant. & Yes $\square$ \\
\hline I wish to receive a summary of the results from the study (please circle). & Yes / \\
\hline
\end{tabular}


Declaration by participant:

I hereby consent to take part in this study.

Participant's name:

Signature:

Date:

Declaration by member of research team:

I have given a verbal explanation of the research project to the participant, and have answered the participant's questions about it.

I believe that the participant understands the study and has given informed consent to participate.

Researcher's name: 
Appendix XII: Publisher permissions to reproduce journal articles 
Study One: Journal of the Royal Society of Medicine: Open

Venous thromboembolism risk associated with protracted work- and computer-related seated immobility: a case-control study.

\section{Journal Authors}

The following SAGE's Journal Author Reuse Policy, effective as of March 20, 2013:

- You may do whatever you wish with the version of the article you submitted to the journal (Version 1).

- Once the article has been accepted for publication, you may post the accepted version (Version 2) of the article on your own personal website, your department's website or the repository of your institution without any restrictions.

- You may not post the accepted version (Version 2) of the article in any repository other than those listed above (i.e. you may not deposit in the repository of another institution or a subject-matter repository) until 12 months after publication of the article in the journal.

- You may use the published article (version 3) for your own teaching needs or to supply on an individual basis to research colleagues, provided that such supply is not for commercial purposes.

- You may use the article (version 3) in a book authored or edited by you at any time after publication in the journal.

- You may not post the published article (version 3) on a website or in a repository without permission from SAGE.

- When posting or re-using the article please provide a link to the appropriate DOI for the published version of the article on SAGE Journals (http://online.sagepub.com)

All commercial or any other re-use of the published article should be referred to SAGE. More information can be found at: http://www.sagepub.com/journalsPermissions. nav. 
From:

Sent:

To:

Subject:
PermissionsUK <Permissions@sagepub.co.uk>

Saturday, 23 July 2016 3:56 AM

Irene Braithwaite

RE: Permission to use article accepted for publication, but not yet published:

JRSMO-15-043.R1

Dear Irene Braithwaite,

Thank you for the information. The journal editors have replied to me and informed me that your article is slated to

be published in the August journal issue. I am also pleased to report we can grant your request without a fee as part

of your thesis.

Please accept this email as permission for your request as detailed below. Permission is granted for the life of the edition on a non-exclusive basis, in the English language, throughout the world in all formats provided full citation is made to the original SAGE publication. If your thesis will be published before the article, then we request that the notice "published ahead of print" be included with your citation to the source.

The permission is subject to approval from any co-authors on the original project. Please note approval excludes any graphs, photos, excerpts, etc. which required permission from a separate copyright holder at the time of publication. If your material includes anything which was not your original work, please contact the rights holder for permission to reuse those items.

If you have any questions, pelase let me know.

Best Wishes,

Craig Myles

on behalf of SAGE Ltd. Permissions Team

SAGE Publishing

1 Oliver's Yard, 55 City Road

London, EC1Y 1SP

UK

WwW.sagepublishing.com

SAGE Publications Ltd, Registered in England No.1017514

Los Angeles | London | New Delhi

Singapore | Washington DC

The natural home for authors, editors \& societies

Thank you for considering the environment before printing this email. 
Study Four: Postgraduate Medical Journal.

Lower limb immobilisation and venous thromboembolism risk: combined case-control studies

BMJ PUBLISHING GROUP LTD. LICENSE
TERMS AND CONDITIONS

This Agreement between Medical Research Institute of New Zeal and -- Irene Braithwaite ("You") and BMJ Publishing Group Ltd. ("BMJ Publishing Group Ltd.") consists of your license details and the terms and conditions provided by BMJ Publishing Group Ltd. and Copyright Clearance Center.

License Number 3990010930868

License date Nov 15, 2016

Licensed Content Publisher BMJ Publishing Group Ltd.

Licensed Content Publication Postgraduate Medical Journal

Licensed Content Title Lower limb immobilisation and venous thromboembollsm risk:

$\begin{array}{ll}\text { Licensed Content Author } & \begin{array}{l}\text { Irene Braithwaite, Bridget Healy, Laird Cameron, Mark Weatherall, } \\ \text { Richard Beasley }\end{array}\end{array}$

Licensed Content Date Oct 26, 2016

Type of Use Dissertation/Thesis

$\begin{array}{ll}\text { Rype of Use } & \text { Author of this article } \\ \text { Requestor type } & \text { Pint }\end{array}$

Format Print and electronic

Portion Figure/table/extract

Number of figure/table

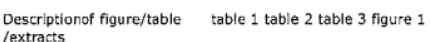

Will you be translating?

Circulation/distribution

Order reference number

Titie of your thesis /
dissertation

Expected completion date

Estimated size(pages)

Requestor Location

No

Thesis PhD

Lower limb immoblity and venous thromboembolism risk :

investigating preventive measures

Nov 2016

352

Medical Research Institute of New Zealand

Level 7, CSB
Wellington Hospital

Wellington Hospita
Newtown,

Wellington, 6021

New Zealand
Attn: Irene Braithwa

Publisher Tax ID GB674738491

Billing Type Invoice

Billing Address Medical Research Institute of New Zealand

Level 7, CSB

Wellington Hospital

Newtown

Wellington, New Zealand 6021
Attn: Irene Braithwaite

Total

$0.00 \mathrm{GBP}$

Terms and Conditions

BMJ Group Terms and Conditions for Permissions

When you submit your orderyou are subject to the terms and conditions set out below. You will also haveagreed to the Copyright Clearance Center's ("CCC") terms and conditionsregarding billing and payment https:/s/ 100.copyright.com/App/PaymentTermsAndConditions.jsp. CCC are acting as the BMJ Publishing Group Limited's ("BMJ Group's") agent.

Subject to the terms set outherein, the BMJ Group hereby grants to you (the Licensee) a

non-exclusive,non-transferable licence to re-use material as detailed in your request forthis/those purpose(s) only and in accordance with the following conditions:

1) Scope of Licence: Useof the Licensed Material(s) is restricted to the ways specified by you duringthe order process and any additional use(s) outside of those specified in thatrequest, require a further grant of permission.

2) Acknowiedgement: Inall cases, due acknowledgement to the original publication with permission fromthe BMJ Group should be stated adjacent to the reproduced Licensed Material. The format of such acknowledgement should read as follows

"Reproduced from [publicationtitle, author(s), volume number, page numbers, copyright notice year] withpermission from BMJ Publishing Group Ltd."

3) Third Party Material:BMJ Group acknowledges to the best of its knowledge, it has the rights to 
licenceyour reuse of the Licensed Material, subject always to the caveat thatimages/diagrams, tables and other illustrative material included within, whichhave a separate copyright notice, are presumed as excluded from the licence. Therefore,you should ensure that the Licensed Material you are requesting is original toBMJ Group and does not carry the copyright of another entity (as credited inthe published version). If the credit line on any part of the material you haverequested in any way
indicates that it was reprinted or adapted by BMJ Groupwith permission from another source, then you should seek permission from thatsource directly to re-use the Licensed Material, as this is you should seek permission from thatsou
outside of thelicence granted herein.

4) Altering/ModifyingMaterial: The text of any material for which a licence is granted may notbe altered in any way without the prior express permission of the BMJ Group. Subject to Clause 3 above however, single figure adaptations do not require BMJGroup's approval; however, the adaptation should be credited as follows:

"Adapted by permission fromBMJ Publishing Group Limited. [publication title, author, volume number, pagenumbers, copyright notice year]

5) Reservation of Rights:The BMJ Group reserves all rights not specifically granted in the combinationof (i) the licence details provided by you and accepted in the course of thislicensing transaction, (ii) these tems and conditions and (iii) CCC's Billing and Payment Tems and Conditions.

6) Timing of Use: Firstuse of the Licensed Material must take place within 12 months of the grant ofpermission.

7). Creation of Contractand Termination: Once you have submitted an order via Rightslink and thisis received by $\mathrm{CCC}$, and subject to you completing accurate details of yourproposed use, this is when a binding contract is in effect and our acceptanceoccurs. As you are ordering rights from a periodical, to the fullest extentpermitted by law, you will have no right to cancel the contract from periodical, to the fullest extentpermitted by law, you will have no right to cancel the contract from
this pointother than for BMJ Group's material breach or fraudulent misrepresentation oras otherwise permitted under a statutory right. Payment must be made inaccordance with CCC's Billing and permitted under a statutory right. Payment must be made inaccordance with CCC's Billing and
Payment Terms and conditions. In the eventthat you breach any material condition of these terms and condition or any of CCC's Billing and Payment Terms and Conditions, the license is automatically terminated uponwritten notice from the BMJ Group or CCC or as otherwise provided for in CCC's Billing and Payment Terms and Conditions, where these apply. Continued use of materials wherea licence has been terminated, as well as any use of the Licensed Materialsbeyond the scope of an unrevoked licence, may constitute intellectual propertyrights infringement and BMJ Group reserves the right to take any and all actionto protect its intellectual property rights in the Licensed Materials.

8. Warranties: BMJGroup makes no express or implied representations or warranties with respect tothe Licensed Material and to the fullest extent permitted by law this isprovided on an "as is" basis. For the avoidance of doubt BMJ Group does notwarrant that the Licensed Material is accurate or fit for any particularpurpose.

9. Limitation ofLiability: To the fullest extent permitted by law, the BMJ Group disclaimsall liability for any indirect, consequential or incidental damages (includingwithout limitation, damages for loss of profits, information or interruption)arising out of the use or inability to use the Licensed Material or theinability to obtain additional rights to use the Licensed Material. To thefullest extent permitted by law, the maximum aggregate liability of the BMJGroup for any claims, costs, permitted by law, the maximum aggregate liability of the BMJGroup for any claims, costs,
proceedings and demands for direct losses causedby BMJ Group's breaches of its obligations herein shall be limited to twice theamount paid by you to CCC for the licence granted herein.

10. Indemnity: You hereby indemnify and holdharmless the BMJ Group and their respective officers, directors, employees andagents, from and against any and all claims, costs, proceeding or demands arisingout of your unauthorised use of the Licensed Material.

11. No Transfer of License: This licence ispersonal to you, and may not be assigned or transferred by you without priorwritten consent from the BMJ Group or its authorised agent(s). BMJ Groupmay assign or transfer any of its tights and obligations under this Agreement,upon written notice to you.

12. No Amendment Except inWriting: This licence may not be amended except in a writing signed by bothparties (or, in the case of BMJ Group, by CCC on the BMJ Group's behalf).

13. Objection to Contraryterms: BMJ Group hereby objects to any terms contained in any purchaseorder, acknowledgment, check endorsement or other writing prepared by you, which terms are inconsistent with these terms and conditions or CCC's Billing and Payment Terms and Conditions. These terms and conditions, together with $\mathrm{CCC}^{\prime} \mathrm{s}$ Billing and Payment Terms and Conditions (which to the extent they are consistent areincorporated herein), comprise the entire agreement between you and BMJ Group (and CCC) and the Licensee concerning this licensing transaction. In the event ofany conflict between your obligations established by these terms and conditionsand those established by CCC's Billing and Payment Terms and Conditions, theseterms and conditions shall control.

14. Revocation: BMJGroup or CCC may, within 30 days of issuance of this licence, deny thepermissions described in this licence at their sole discretion, for any reasonor no reason, with a full refund payable to you should you have no ben able to exectse your rights in fill Notce of such denal will be you using thect notice from BMJGroup or CCC will not, to the fullest extent permitted by law alter orinvalidate the denial. For the fullest extent permitted by law in no event willBMJ Group or CCC be responsible or liable for any costs, expenses or damagencured by you as a result of a denial of your permission request, other thana refund of the amount(s) paid by you to BMJ Group and/or CCC for denied permissions

15. Restrictions to thelicense: 
15.1 Promotion: BMJ Group will not give permission to reproduce infull or in part any Licensed Material for use in the promotion of thefollowing

a) non-medical products thatare hannful or potentially harmful to health: alcohol, baby milks and/or, sunbeds

b) medical products that donot have a product license granted by the Medicines and Healthcare productsRegulatory Agency (MHRA) or its international equivalents. Marketing of theproduct may start only after data sheets have been released to members of themedical profession and must start only atter data sheets have been released to members of themedical
conform to the marketing authorization contained inthe product license.

16. Translation: This permission is granted for non-exclusive worldEnglish language rights only unless explicitly stated in your licence. Iftranslation rights are granted, a professional translator should be employedand the content should be reproduced word for word preserving the integrity ofthe content.

17. General:Neither party shall be liable for failure, default or delay in performingits obligations under this Licence, caused by a Force Majeure event which shallinclude any act of God, war, or threatened war, act or threatened act ofterrorism, riot, strike, lockout, individual action, fire, flood, drought, tempest or other event beyond the reasonable control of either party

17.1 Inthe event that any provision of this Agreement is held to be invalid, theremainder of the provisions shall continue in full force and effect.

17.2 Thereshall be no right whatsoever for any third party to enforce the terms andconditions of this Agreement. The Parties hereby expressly wish to exclude theoperation of the Contracts (Rights of Third Parties) Act 1999 and any otherlegislation which has this effect and is binding on this agreement.

17.3 Tothe fullest extent permitted by law, this Licence will be governed by the lawsof England and shall be governed and construed in accordance with the laws of England. Any action arising out of or relating to this agreement shall be brought in courtssituated in England save where it is necessary for BMJ Group for enforcement tobring proceedings to bring an action in an alternative jurisdiction 


\section{Study Four: Occupational Ergonomics}

Legflow increases lower limb venous haemodynamics when seated: a randomised controlled trial

Irene Braithwaite

From:

Sent:

To:

Cc:

Subject:

\author{
Biman Das <Biman.Das@Dal.Ca> \\ Friday, 1 July 2016 7:30 AM \\ Irene Braithwaite \\ Marion Lilley \\ Permission to use publication
}

Dear Irene,

I am pleased to receive your e-mail of June 29,2016 . It will be in order to include your publication from Occupational Ergonomics (Vol. 12, No.4, 2015, pg. 131-140) in your PhD Dissertation, since you are the principal researcher. With this e-mail, I am also informing the publisher, IOS press, Ms. Marion Lilley.

Best wishes and regards.

Sincerely,

Biman 


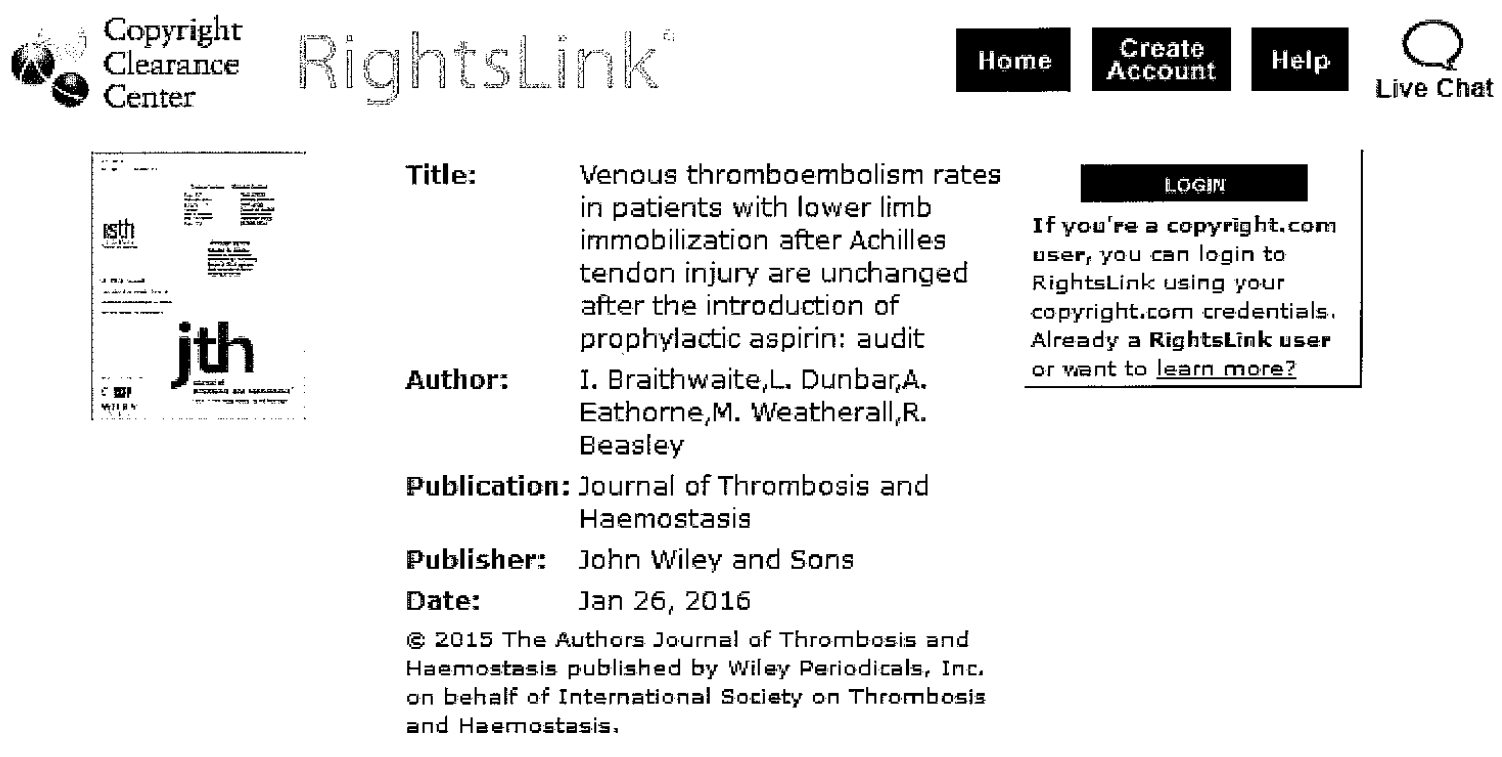

\section{Welcome to RightsLink}

This article is available under the terms of the Creative Commons Attribution Non-Commercial No Derivatives License CC BY-NC-ND (which may be updated from time to time) and permits non-commercial use, distribution and reproduction in any medium, without alteration, provided the original work is properly cited and it is reproduced yerbatim.

For an understanding of what is meant by the terms of the Creative Commons License, please refer to wilev's Open Access Terms and Conditions.

Permission only needs to be obtained for commercial use.

John wiley and Sons has partnered with Copyright Clearance Center's RightsLink service to offer a variety of options for reusing John wiley and Sons content. Select the "I would like to..." drop-down menu to view the many reuse aptions available to you.

If you wish to adapt, alter, translate or create any other derivative work from this article, permission must be sought from the Publisher. Please enail your requirements to RiahtsLink@ilew.com.

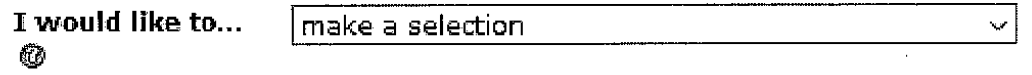




\section{Study Eight: BMJ Open}

Cohort feasibility study of an intermittent pneumatic compression device within a belowknee cast for the prevention of venous thromboembolism

BMJ PUBLISHING GROUP LTD. LICENSE

TERMS AND CONDITIONS

This Agreement between Medical Research Institute of New Zealand -- Irene Braithwaite ("You") and BMJ Publishing Group Ltd. ("BMJ Publishing Group Ltd.") consists of your license details and the terms and conditions provided by BMJ Publishing Group Ltd. and Copyright Clearance Center.

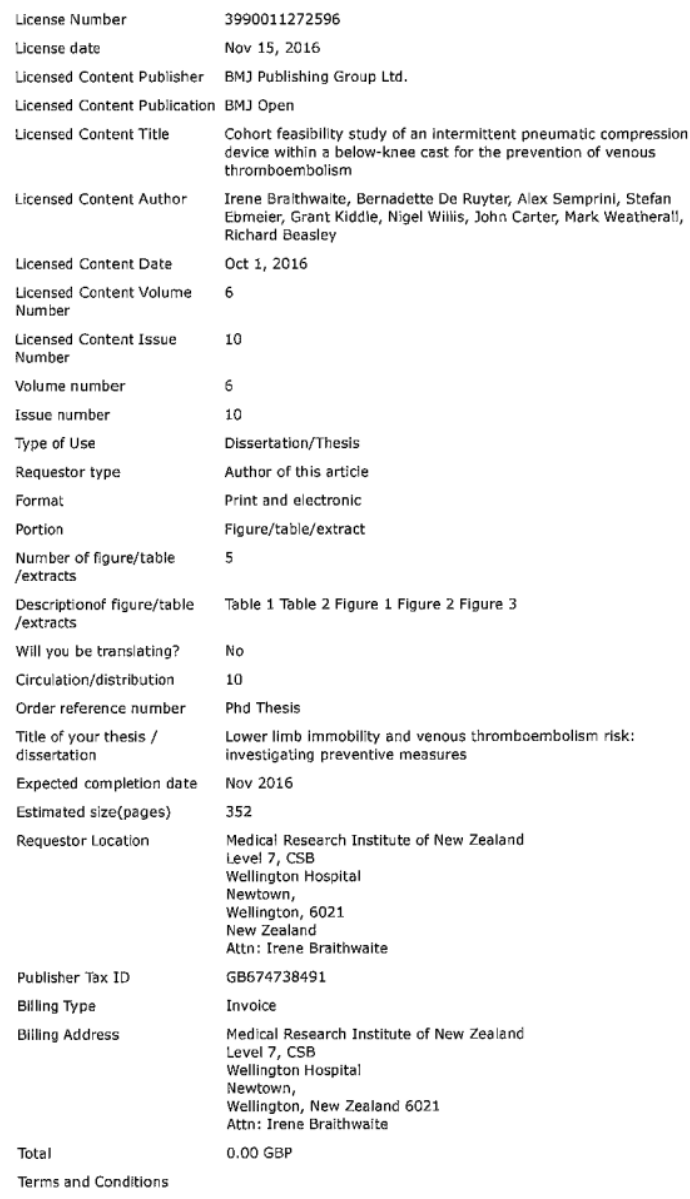

When you submit your orderyou are subject to the terms and conditions set out below. You will also haveagreed to the Copyright Clearance Center's ("CCC") ierms and conditionssegarding billing ant payment hitps://s100.copyright.com/App/PaymentTermsAndConditions.jsp. CCC are acting as the BMJ Publishing Group Limited's ("BMJ Group's") agent.

Subject to the terms set outherein, the BMJ Group hereby grants to you (the Licensee) a

non-exclusive,non-transferabie licence to re-use material as detailed in your request forthis/those

purpose(s) only and in accordance with the following condition

1) Scope of Licence: Useof the Licensed Material(s) is restricted to the ways specified by you 
duringthe order process and any additional use(s) outside of those specified in thatrequest, require a further grant of permission.

2) Acknowledgement: Inall cases, due acknowledgement to the original publication with permission fromthe BMJ Group should be stated adjacent to the reproduced Licensed Material. The format of such acknowledgement should read as follows:

"Reproduced from [publicationtitle, author(s), volume number, page numbers, copyright notice year] withpermission from BMJ Publishing Group Ltd

3) Third Party Material:BMJ Group acknowledges to the best of its knowledge, it has the rights to licenceyour reuse of the Licensed Material, subject always to the caveat thatimages/diagrams, tables and other illustrative material included within, whichhave a separate copyright notice, are presumed as excluded from the licence. Therefore,you should ensure that the Licensed Material you are requesting is original toBMJ Group and does not carry the copyright of another entity (as credited inthe published version). If the credit line on any part of the material you haverequested in any way indicates that it was reprinted or adapted by BMJ Groupwith permission from another source, then you should seek permission from thatsource directiy to re-use the Licensed Material, as this is outside of thelicence granted herein.

4) Altering/ModifyingMaterial: The text of any material for which a licence is granted may notbe altered in any way without the prior express permission of the BMJ Group. Subject to Clause 3 above however, single figure adaptations do not require BMJGroup's approval; however, the adaptation should be credited as follow:

"Adapted by permission fromBMJ Publishing Group Limited. [publication title, author, volume number, pagenumbers, copyright notice year

5) Reservation of Rights:The BMJ Group reserves all rights not specifically granted in the combinationof (i) the licence details provided by you and accepted in the course of thislicensin Condition

6) Timing of Use: Firstuse of the Licensed Material must take place within 12 months of the gran ofpermission

7). Creation of Contractand Termination: Once you have submitted an order via Rightslink and thisis received by $\mathrm{CCC}$, and subject to you completing accurate details of yourproposed use, this is when a binding contract is in effect and our acceptanceoccurs. As you are ordering rights from a periodical, to the fullest extentpermitted by law, you will have no right to cancel the contract from permitted under a statutory right. Payment must be made inaccordance with CCC's Billing and permitted under a statutory right. Payment must be made inaccordance with CCC's Billing and Payment Tems and conditions. In the eventthat you breach any material condition of thes
and condition or any of CCC's Billing and Payment Terms and Conditions, the license is and condition or any terminated uponwritten notice from the BMJ Group or CCC the license is for in CCC's Billing and Payment Terms and Conditions, where these apply.. Continued use of materials where licence has been terminated, as well as any use of the Licensed Materialsbeyond the scope of an unrevoked licence, may constitute intellectual propertyrights infringement and $B M$ Group reseres the Licensed Materials.

8. Warranties: BMJGroup makes no express or implied representations or warranties with respect tothe Licensed Material and to the fullest extent permitted by law this isprovided on an "as is" basis. For the avoidance of doubt BMJ Group does notwarrant that the Licensed Material is accurate or fi for any particularpurpose

9. Limitation ofLiability: To the fullest extent permitted by law, the BMJ Group disclaimsall liability for any indirect, consequential or incidental damages (includingwithout limitation, damages for loss of profits, information or interruption)arising out of the use or inability to use the Licensed Material or theinability to obtain additional rights to use the Licensed Material. To thefullest extent permitted by law, the maximum aggregate liability of the BMJGroup for any claims, costs, proceedings and demands for direct losses causedby BMJ Group's breaches of its obligations herein shall be limited to twice theamount paid by you to $\mathrm{CCC}$ for the licence granted herein.

10. Indemnity: You hereby indemnify and holdharmless the BMJ Group and their respective officers, directors, employees andagents, from and against any and all claims, costs, proceeding or demands arisingout of your unauthorised use of the Licensed Material.

11. No Transfer of License: This licence ispersonal to you, and may not be assigned or transferred by you without priorwritten consent from the BMJ Group or its authorised agent(s). BMJ Groupmay assign or transfer any of its rights and obligations under this Agreement,upon written notice to you.

12. No Amendment Except inWriting: This licence may not be amended except in a writing signed by bothparties (or, in the case of BMJ Group, by CCC on the BMJ Group's behalf).

13. Objection to Contraryterms: BMJ Group hereby objects to any terms contained in any purchaseorder, acknowledgment, check endorsement or other writing prepared by you, which terms are inconsistent with these terns and conditions or CCC's Billing and Payment Terms and Condtions. These terms and conditions, together with CCC's Billing and Payment Terms and Condilons (which to the enter agreenent beth transaction. Tn the event of and conditions shall control.

14. Revocation: BMJGroup or CCC may, within 30 days of issuance of this licence, deny thepermissions described in this licence at their sole discretion, for any reasonor no reason, with a 
full refund payable to you should you have not been ableto exercise your rights in full. Notice of such denial will be made using thecontact information provided by you. Failure to receive such notice from BMJGroup or CCC will not, to the fullest extent permitted by law alter orinvalidate the denial. For the fullest extent permitted by law in no event willBMJ Group or CCC be responsible or liable for any costs, expenses or damageincurred by you as a result of a denial of your permission request, other thana refund of the amount(s) paid by you to BMJ Group and/or CCC for denied permissions.

15. Restrictions to thelicense

15.1 Promotion: BMJ Group will not give permission to reproduce infull or in part any Licensed Material for use in the promotion of thefollowing:

a) non-medical products thatare harmful or potentially harmful to health: alcohol, baby milks and/or, sunbeds

b) medical products that donot have a product license granted by the Medicines and Healtheare productsRegulatory Agency (MHRA) or its international equivalents. Marketing of theproduct may start only after data sheets have been released to members of themedical profession and must conform to the marketing authorization contained inthe product license.

16. Translation: This permission is granted for non-exclusive worldEnglish language rights only unless explicitly stated in your licence. Iftranslation rights are granted, a professional translator should be employedand the content should be reproduced word for word preserving the integrity ofthe content.

17. General:Neither party shall be liable for failure, default or delay in performingits obligations under this Licence, caused by a Force Majeure event which shallinclude any act of God, war, or threatened war, act or threatened act ofterrorism, riot, strike, lockout, individual action, fire, flood, drought,tempest or other event beyond the reasonable control of either party.

17.1 Inthe event that any provision of this Agreement is held to be invalid, theremainder of the provisions shall continue in full force and effect.

17.2 Thereshall be no right whatsoever for any third party to enforce the terms andconditions of this Agreement. The Parties hereby expressly wish to exclude theoperation of the Contracts (Rights of Third Parties) Act 1999 and any otherlegislation which has this effect and is binding on this agreement.

17.3 Tothe fullest extent permitted by law, this Licence will be governed by the lawsof England and shall be governed and construed in accordance with the laws of England. Any action arising out of or relating to this agreement shall be brought in courtssituated in England save where it is necessary for BMJ Group for enforcement tobring proceedings to bring an action in an alternative jurisdiction 


\section{References}

Aldington S, Pritchard A, Perrin K, Jame, K, Wijesinghe M, Beasley R. Prolonged seated immobility at work is a common risk factor for venous thromboembolism leading to hospital admission. Intern Med J.2008;38(2):133-135.

Anderson Jr. F, Wheeler H, Goldberg R, Hosmer D, Patwardhan N, Jovanovic B et al. A PopulationBased Perspective of the Hospital Incidence and Case-Fatality Rates of Deep Vein Thrombosis and Pulmonary Embolism. Arch Intern Med.1991;151(5):933-938.

Antiplatelet Trialists' Collaboration. Collaborative overview of randomised trials of antiplatelet therapy - III: reduction in venous thrombosis and pulmonary embolism by antiplatelet prophylaxis among surgical and medical patients. BMJ. 1994:308:235-246.

Asano H, Matsubara M, Suzuki K, Morita S, Shinomiya K. Prevention of pulmonary embolism by a foot sole pump. J Bone Joint Surg Br. 2001;83-B(8):1130-2.

Ashrani A, Heit J. Incidence and cost burden of post-thrombotic syndrome. J Thromb Thrombolysis. 2009;28(4):465-476.

Bayer New Zealand Limited. (2015). DATA SHEET XARELTO ${ }^{\circledR}$ (Rivaroxaban). Bayer New Zealand Limited.

Beasley R, Heuser P, Raymond N. SIT (Seated immobility thromboembolism) syndrome: a 21st Century lifestyle hazard. N Z Med J. 2005;118(1212):U1376.

Beasley R, Raymond N, Hill S, Nowitz M Hughes R. eThrombosis: the 21st century variant of venous thromboembolism associated with immobility. Eur Respir J. 2003;21(2):374-376.

Beckman M, Hooper W, Critchley S, Ortel T. Venous Thromboembolism. A Public Health Concern. Am J Prev Med. 2010;38(4 SUPPL.):S495-S501.

Beebe-Dimmer J, Pfeifer J, Engle J, Schottenfeld D. The epidemiology of chronic venous insufficiency and varicose veins. Ann Epidemiol. 2005;15(3):175-184.

Belcaro G, Geroulakos G, Nicolaides A, Myers K, Winford M. Venous thromboembolism from air travel: the LONFLIT study. Angiolog. 2001;52(6):369-374.

Bergqvist D, Benoni G, Bjorgell O, Fredin $\mathrm{H}$, Hedlunch U, Nicolas S, et al. Low Molecular Weight Heparin (enoxaparin) as Prophylaxis against Venous Thromboembolism after Total Hip Replacement. N Eng J Med. 1996;335(10):696-700.

Biddle S, Edwardson C, Wilmot E, Yates T, Gorely T, Bodicoat D et al. A Randomised Controlled Trial to Reduce Sedentary Time in Young Adults at Risk of Type 2 Diabetes Mellitus : Project STAND (Sedentary Time ANd Diabetes). PLoS ONE. 2015;10(12):e0143398. 
Blom J, Doggen C, Osanto S, Rosendaal F. Malignancies, Prothrombotic Mutations and the Risk of Venous Thrombosis. JAMA. 2005;93(6):715-722.

Bovill EG, van der Vliet A. Venous valvular stasis-associated hypoxia and thrombosis: what is the link? Annu Rev Physiol. 2011;3:527-545.

Bradley J, Krugener $\mathrm{G}$, Jager $\mathrm{H}$. The effectiveness of intermittent plantar venous compression in prevention of deep venous thrombosis after total hip arthroplasty. J Arthroplasty. 1993;8(1):57-61.

Braithwaite I, Dunbar L, Eathorne A, Weatherall M, Beasley R. Venous thromboembolism rates in lower limb immobilization after Achilles tendon injury unchanged after introduction of prophylactic aspirin: audit. J Thromb Haemost. 2016;14:331-335.

Braithwaite I, Mackintosh S, Mallon A, Weatherall M, Beasley R. Legflow increases lower-limb venous haemodynamics when seated: A randomised controlled trial. Occupational Ergonomics. 2016;12(4):DOI 10.3233/OER-150231.

Braithwaite I, Maher B, Cameron L, Weatherall M, Beasley R. Venous thromboembolism risk associated with protracted work- and computer-related immobility: a case-control study. JRSM Open. 2016 accepted for publication.

Brown WJ, Miller YD, Miller R. Sitting time and work patterns as indicators of overweight and obesity in Australian adults. Int J Obes Relat Metab Disord. 2003;27(11):1340-1346.

Brownson RC, Boehmer TK, Luke DA. Declining rates of physical activity in the United States: What Are the Contributors? Annu Rev Public Health. 2005;26:421-443.

Cannegieter SC. Thrombosis Prophylaxis During Plaster Cast Lower Leg Immobilisation (Pot-Cast). Posted online 2012. Retrieved June 18, 2016, from https://clinicaltrials.gov/ct2/show/record/NCT01542762?term=lower+limb+immobilisation\&ra $\mathrm{nk}=4$

Cannegieter SC, Doggen CJ, van Houwelingen HC, Rosendaal FR. Travel-related venous thrombosis: results from a large population-based case control study (MEGA study). PLoS Med. 2006;3(8): e307.

Caprini J, Chucker J, Zuckerman L, Vagher J, Franck C, Cullen J. Thrombosis prophylaxis using external compression. Surg Gynaecol Obstet. 1983;156: 599-604.

Carrothers A, Rodriguez-Elizalde S, Rogers B, Razmjou H, Gollish J, Murnaghan J. Patient-reported compliance with thromboprophylaxis using an oral factor Xa inhibitor (Rivaroxaban) following total hip and total knee arthroplasty. Journal of Arthroplasty. 2014;29(7):1463-1467.

Caschman J, Blagg S, Bishay M. The efficacy of the A-V Impulse system in the treatment of posttraumatic swelling following ankle fracture: a prospective randomized controlled study. Journal Orthop Trauma. 2004;18(9):596-601. 
Chang $\mathrm{HCL}$, BurbridgeH, Wong C. Extensive deep vein thrombosis following prolonged gaming ('gamer's thrombosis'): a case report. J Med Case Rep. 2013;7(1):235.

Charalambous C, Cleanthous S, Tryfonidis M, Goel A, Swindell R, Ellis D. Foot pump prophylaxis for deep venous thrombosis - Rate of effective usage following knee and hip arthroplasty. Int Orthop. 2003;27(4):208-210.

Charles T, Mackintosh S, Fingleton J, Braithwaite I, Weatherall M, Beasley R. Efficacy of micromobile foot compression device in increasing lower limb venous blood flow. Int J Vasc Med. 2013;2013:948769.

Chau JY, Grunseit AC, Chey T, Stamatakis E, Brown WJ, Matthews CE et al. Daily sitting time and allcause mortality: a meta-analysis. PLoS ONE. 2013;8(11):e80000.

Chew H. Shortness of breath in a computer gamer. Am J Emerg Med. 2006;24(7):892-894.

Clark BK, Sugiyama T, Healy GN, Salmon J, Dunstan DW, Owen N. Validity and reliability of measures of television viewing time and other non-occupational sedentary behaviour of adults: a review. Obes Rev. 2009;10(1):7-16.

Clarkson S. How sitting at a desk for too long could give you a deadly blood clot. Daily Mail UK. 05 July 2011. Health Section.

Clements R, Churilov L, Wahab A, Ng L. Exploratory analysis of factors associated with venous thromboembolism in Victorian acute traumatic spinal cord-injured patients 2010-2013. Spinal Cord. 2016:epub ahead of print.

Clemes S, Patel R, Mahon C, Griffiths P. Sitting time and step counts in office workers. Occupational Medicine. 2014;64(3):188-192.

Cohen A, Agnelli G, Anderson F, Arcelus J, Bergqvist D, Brecht J et al. Venous thromboembolism (VTE) in Europe: The number of VTE events and associated morbidity and mortality. Thromb Haemost. 2007;98(4):756-764.

Cohen A, Quinlan D. Correspondence: PEP trial. Lancet. 2000;356:247.

Cohen AT, Bailey CS, Alikhan R, Cooper DJ. Letter to the Editor: Extended thromboprophylaxis with low molecular weight heparin reduces symptomatic venous thromboembolism following lower limb arthroplasty - A meta-analysis. Thromb Haemost. 2001;85(5):940-941.

Colwell CW, Froimson MI, Mont MA, Ritter MA, Trousdale RT, Buehler KC et al. Thrombosis Prevention After Total Hip Arthroplasty: A Prospective, Randomized Trial Comparing a Mobile Compression Device with Low-Molecular-Weight Heparin. J Bone Joint Surg Am. 2010:92(3):527-535.

Comerota AJ, Chouhan V, Harada RN, Sun L, Hosking J, Veermansunemi R et al. The fibrinolytic effects of intermittent pneumatic compression: mechanism of enhanced fibrinolysis. Ann Surg. 1997:226(3):306-13. 
Comerota AJ, Katz ML, White JV. Why does prophylaxis with external pneumatic compression for deep vein thrombosis fail? Am J Surg. 1992:164(3):265-268.

Coon W. Risk factors in pulmonary embolism. Surg Gynaecol Obstet. 1996;143(3):385-90.

Coon W, Willis III P, Keller J. Venous Thromboembolism and Other Venous Disease in the Tecumseh Community Health Study. Circulation. 1973;48(4):839-846.

Cruickshank J, Gorlin R, Jennett B. Air travel and thrombotic episodes: the economy class syndrome. Lancet. 1998;2(8609):497-498.

Cushman M, Tsai A, White R, Heckbert S, Rosamond W, Enright P, Folsom A. Deep vein thrombosis and pulmonary embolism in two cohorts: the longitudinal investigation of thromboembolism etiology. Am J Med. 2004:117(1):19-25.

Darvall KAL, Sam RC, Silverman SH, Bradbury AW, Adam DJ. Obesity and Thrombosis. Eur J Vasc Endovasc Surg. 2007;33(2):223-233.

De Groot P, Bleeker MWP, Hopman MTE. Ultrasound: a reproducible method to measure conduit vein compliance. J Appl Physiol (1985). 2005;98(5):1878-1883.

Delcroix M, Lang I, Pepke-Zaba J, Jansa P, D’Armini A, Snijder R et al. Long-Term Outcome of Patients With Chronic Thromboembolic Pulmonary Hypertension (CTEPH): Results From an International Prospective Registry. Circulation. 2016:133(9):859-71.

Delis KT, Slimani G, Hafez HM, Nicolaides AN. Enhancing venous outflow in the lower limb with intermittent pneumatic compression. A comparative haemodynamic analysis on the effect of foot vs. calf vs. foot and calf compression. Eur J Vasc Endovasc Surg. 2000:19(3):250-260.

Dellinger R, Carlet J, Masur H, Gerlach H, Calandra T, Cohen J et al. Surviving Sepsis Campaign guidelines for management of severe sepsis and septic shock. Intensive Care Med. 2004:30(4):536-55.

Dickson B. Historical Review Venous Thrombosis: On the History of Virchow's Triad. Univ Toronto Med J. 2004;81(3):166-171.

Domeij-Arverud E, Labruto F, Latifi A, Nilsson G, Edman G, Ackermann PW. Intermittent pneumatic compression reduces the risk of deep vein thrombosis during postoperative lower limb immobilisation: A prospective randomised trial of acute ruptures of the Achilles tendon. Bone Joint J. 2015;97-B(5):675-680.

Domeij-Arverud, E, Latifi A, Labruto F, Nilsson G, Ackermann P. (2013). Can foot compression under a plaster cast prevent deep-vein thrombosis during lower limb immobilisation? Bone Joint J. 2013;95-B(9):1227-1231.

Dugdill L, Brettle A, Hulme C, McCluskey S, Long, A. (2008). Workplace physical activity interventions: a systematic review. International Journal of Workplace Health Management, 2008;1(1):20-40. 
Dunstan D, Barr E, Healy G, Salmon J, Shaw J, Balkau B et al. Television viewing time and mortality: The australian diabetes, obesity and lifestyle study (ausdiab). Circulation. 2010;121(3):384-391.

Edwards J, Pulido P, Ezzet K, Copp S, Walker R, Colwell C. (2008). Portable Compression Device and Low-Molecular-Weight Heparin Compared With Low-Molecular-Weight Heparin for Thromboprophylaxis After Total Joint Arthroplasty. J Arthroplasty. 2008;3(8):1122-1127.

Egeberg O. Inherited antithrombin deficiency causing thrombophilia. Thrombosis Diath Haemorr. 1965;15(13):516-30.

Elikowski W, Kurosz J, Zawilska K. "eThrombosis" - zatorowa sprowokowana przez wielogodzinną pozycję siedzącą przed komputerem. Kardiologia Polska. 2011;69(4):377-381. [Article in Polish]

Engbers M, Blom J, Cushman M, Rosendaa, F, van Hylckama Vlieg A. The contribution of immobility risk factors to the incidence of venous thrombosis in an older population. $J$ Thromb Haemost. 2014;12(3):290-296.

Eriksen D, Rosth $\varnothing \mathrm{j}$ S, Burr H, Holtermann A. Sedentary work-Associations between five-year changes in occupational sitting time and body mass index. Preventive Medicine. 2015;73:1-5.

Eriksson B, Borris L, Friedman R, Haas S, Huisman M, Kakkar A et al. Rivaroxaban versus Enoxaparin for thromboprophylaxis after hip arthroplasty. New Engl J Med. 2008;358(26):2765-75.

Eriksson BI, Kakkar AK, Turpie AG, Gent M, Bandel TJ, Homering M et al. Oral rivaroxaban for the prevention of symptomatic venous thromboembolism after elective hip and knee replacement. J Bone Joint Surg [Br]. 2009;91-B:636-644.

Ettema H, Kollen B, Verheyen C, Büller H. Prevention of venous thromboembolism in patients with immobilization of the lower extremities: a meta-analysis of randomized controlled trials. $J$ Thromb Haemost. 2008;6(7):1093-1098.

Falck-Ytter Y, Francis C, Johanson N, Curley C, Dahl O, Schulman S et al. Prevention of VTE in Orthopedic Surgery Patients: Antithrombotic therapy and prevention of thrombosis, 9th ed: American College of Chest Physicians Evidence-Based Clinical Practice Guidelines. Chest. 2012;141((2)(Suppl)):e278S-325S.

Ferrari E, Chevallier T, Chapelie, A, Baudouy M. Travel as a risk factor for venous thromboembolic disease: a case-control study. Chest.1999;115(3):440-444.

Ferrari E, Morgan G. Travel as a risk factor for venous thromboembolic disease. Eur J Med Res. 2004;9(3):146-149.

File T. Computer and Internet Use in the United States: population characteristics. Current Population Survey Reports.US Census Bureau. US Department of Commerce. May 2013. Retrieved June 21, 2016 from https://www.census.gov/history/pdf/2013computeruse.pdf

Fitbit. Fitbit.com. Retrieved June 21, 2016, from https://www.fitbit.com/nz/about 
Fordyce MJF, Ling R. A venous foot pump reduces thrombosis after total hip replacement. J Bone Joint Surg [Br]. 1992;74-B(1):45-9.

Fowkes FJ, Price JF, Fowkes FGR. Incidence of diagnosed deep vein thrombosis in the general population: systematic review. Eur J Vasc Endovasc Surg. 2003;25:1-5.

Freemantle N, Calvert M, Wood J, Eastaugh J, Griffin C. Composite Outcomes in Randomized Trials: Greater precision but with greater uncertaintly? JAMA. 2003;289(19):2554-2559.

Gaitini D. Current approaches and controversial issues in the diagnosis of deep vein thrombosis via duplex Doppler ultrasound. J Clin Ultrasound. 2006;34(6):289-297.

Gardner AMN, Fox RH. The venous footpump: influence on tissue perfusion and prevention of venous thrombosis. Ann Rheum Dis. 1992;51:1173-1178.

Geerts W, Bergqvist D, Pineo G, Heit J, Samama C, Lassen M, Colwell C. Prevention of venous thromboembolism: American College of Chest Physicians evidence-based clinical practice guidelines (8th edition). Chest. 208;133(SUPPL.6):381s-453s.

Geerts W, Code K, Jay R, Chen E, Szalai J. A prospective study of venous throbmoembolism after major trauma. New Engl J Med. 1994;331(24):1601-1606.

Geerts W, Pineo G, Heit J, Bergqvist D, Lassen M, Colwell C, Ray J. Prevention of Venous Thromboembolism: The Seventh ACCP Conference on Antithrombotic and Thrombolytic Therapy. Chest. 2004;126:338s-400s.

Gehling H, Giannadakis K, Lefering R, Hessmann M, Achenbach S, Gotzen L. Prospective randomized pilot study of ambulatory prevention of thromboembolism. 2 times $500 \mathrm{mg}$ aspirin (ASS) vs. clivarin 1750 (NMH). Unfallchirurg. 1998;101(1):42-9. [Article in German].

Gherman R, Goodwin T, Leung B, Byrne J, Hethumumi R, Montoro M. Incidence, clinical characteristics, and timing of objectively diagnosed venous thromboembolism during pregnancy. Obstet and Gynecol. 1999;94(5):730-734.

Gillum R. (1987). Pulmonary embolism and thrombophlebitis in the United States, 1970-1985. Am Heart J. 1987;114(5):1262-1264.

Glynn R, Rosner B. (2005). Comparison of risk factors for the competing risks of coronary heart disease, stroke, and venous thromboembolism. Am J Epidemiol. 2005;162(10):975-982.

Goel DP, Buckley R, De Vries G, Abelseth G, Ni A, Gray R. Prophylaxis of deep-vein thrombosis in fractures below the knee: a prospective randomised controlled trial. J Bone Joint Surg [Br]. 2009;91-B(3):388-94.

Goldhaber S, Bounameaux H. Pulmonary embolism and deep vein thrombosis. Lancet. 2012;379(9828):1835-1846.

Grainge M, West J, Card T. Venous thromboembolism during active disease and remission in inflammatory bowel disease: a cohort study. Lancet. 2010;375(9715):657-663. 
Griffiths JT, Matthews L, Pearce CJ, Calder, JDF. (2012). Incidence of venous thromboembolism in elective foot and ankle surgery with and without aspirin prophylaxis. J Bone Joint Surg [Br]. 2012;94-B(2):210-4.

Grodstein F, Stampfer M, Goldhaber S, Manson J, Colditz,G, Speizer F et al. Prospective study of exogenous hormones and risk of pulmonary embolism in women. Lancet. 1996;348(9033):9837.

Hansson P, Eriksson H, Welin L, Svardsudd K, Wilhelmsen L. Smoking and abdominal obesity: risk factors for venous thromboembolism among middle-aged men: the study of men born in 1913. Arch Int Med. 1999;159(16):1886-90.

Haque S, Davies MB. (2015). Oral thromboprophylaxis in patients with ankle fractures immobilized in a below the knee cast. Foot and Ankle Surgery. 2015;21(4):266-268.

Healy B, Beasley R, Weatherall M. (2010). Venous thromboembolism following prolonged cast immobilisation for injury to the tendo Achillis. J Bone Joint Surg [Br]. 2010;92-B(5):646-650.

Healy B, Levin E, Perrin K, Weatherall M, Beasley R. Prolonged work- and computer-related seated immobility and risk of venous thromboembolism. J R Soc Med. 2010;103(11):447-454.

Healy GN, Dunstan DW, Salmon J, Cerin E, Shaw JE, Zimmet PZ, Owen N. Breaks in sedentary time: beneficial associations with metabolic risk. Diabetes Care. 2008;31(4):661-666.

Heit JA. Venous Thromboembolism: Mechanisms, Treatment, and Public Awareness: The Epidemiology of Venous Thromboembolism in the Community. Arterioscler Thromb Vasc Biol. 2008;28:370-372.

Heit JA. Venous thromboembolism: disease burden, outcomes and risk factors. J Thromb Haemost. 2005;3:1611-7.

Heit JA, O'Fallon W, Petterson T, Lohse C, Silverstein M, Mohr D, Melton III L. (2002). Relative impact of risk factors for deep vein thrombosis and pulmonary embolism: a population-based study. Arch Intern Med. 2002;162(11):1245-1248.

Heit JA, Silverstein M, Mohr D, Petterson T, O'Fallon W, Melton III L. Risk factors for deep vein thrombosis and pulmonary embolism: a population-based case-control study. Arch Intern Med. 2000;160(6):809-815.

Hitos K, Cannon M, Cannon S, Garth S, Fletcher JP. Effect of leg exercises on popliteal venous blood flow during prolonged immobility of seated subjects: implications for prevention of travelrelated deep vein thrombosis. J Thromb Haemost.2009;5(9):1890-1895.

Ho K, Tan J. Stratified meta-analysis of intermittent pneumatic compression of the lower limbs to prevent venous thromboembolism in hospitalized patients. Circulation. 2013;128:1003-20.

Holtermann A, Mortensen O, Burr H, Søgaard K, Gyntelberg F, Suadicani P. Fitness, work, and leisure-time physical activity and ischaemic heart disease and all-cause mortalit. Scand J Work Environ Health. 2010;36(5):366-372. 
Homans J. Thrombosis of the Deep Veins of the Lower Leg, Causing Pulmonary Embolism. New Eng/ J Med. 1934;211(22):993-997.

Homans J. Thrombosis of the deep leg veins due to prolonged sitting. New Engl J Med. 1954;250(4):148-9.

Horner D, Hogg K, Body R, Nash M, Mackway-Jones K. The Anticoagulation of Calf Thrombosis (ACT) project: study protocol for a randomized controlled trial. Trials. 2012;13(31). doi:

10.1186/1745-6215-13-31

House of Commons Health Committee. The Prevention of Venous Thromboembolism in Hospitalised Patients: second report of session 2004-05. 2005. House of Commons, United Kingdom.

Huerta C, Johansson S, Wallande M-A, García R. Risk factors and short-term mortality of venous thromboembolism diagnosed in the primary care setting in the United Kingdom. Arch Intern Med. 2007;167:935-943.

Hughes RJ, Hopkins RJ, Hill S, Weatherall M, Van de Water N, Nowitz M et al. Frequency of venous thromboembolism in low to moderate risk long distance air travellers: the New Zealand Air Traveller's Thrombosis (NZATT) study. Lancet. 2003;362(9401):2039-2044.

Hull RD, Raskob GE, Gent M, McLoughlin D, Julian D, Smith FC et al. Effectiveness of intermittent pneumatic leg compression for preventing deep vein thrombosis after total hip replacement. JAMA. 1990;263(17):2313-2317.

IJmker S, Huysmans MA, Blatter BM, van der Beek AJ, van Mechelen W, Bongers PM. (2007). Should office workers spend fewer hours at their computer? A systematic review of the literature. Occup Environ Med. 2007;64(4):211-222.

Jakes RW, Day NE, Khaw K-T, Luben R, Oakes S, Welch A et al. Television viewing and low participation in vigorous recreation are independently associated with obesity and markers of cardiovascular disease risk: EPIC-Norfolk population-based study. Eur J Clin Nutr. 2003;57(9):1089-96.

Jameson SS, Augustine A, James P, Serrano-Pedraza I, Oliver K, Townshend D, Reed MR. (2011). Venous thromboembolic events following foot and ankle surgery in the English National Health Service. J Bone Joint Surg [Br]. 2011;93-B(4):490-497.

Jans MP, Proper KI, Hildebrandt VH. Sedentary behavior in Dutch workers: differences between occupations and business sectors. Am J Prev Med. 2007;33(6):450-454.

John D, Bassett D, Thompson D, Fairbrother J, Baldwin D. Effect of using a treadmill workstation on performance of simulated office work tasks. J Phys Act Health. 2009;6(5):617-624.

Johnson H. Traveller's ankle. BMJ. 1973;3(5871):109.

Jorgensen LN, Wille-Jorgensen P, Hauch O. Prophylaxis of postoperative thromboembolism with low molecular weight heparins. Br J Surg. 1993;80(6):689-704. 
Jorgensen P, Warming T, Hansen K, Paltved C, Vibeke Berg H, Jensen R et al. Low molecular weight heparin (Innohep) as thromboprophylaxis in outpatients with a plaster cast: a venografic controlled study. Thromb Research. 2002;105(6):477-480.

Kabrhel C, Varraso R, Goldhaber SZ, Rimm E, Camargo Jr. CA. Physical inactivity and idiopathic pulmonary embolism in women: prospective study. BMJ. 2011;342:d3867.

Kahn SR, Ducruet T, Lamping D, Arsenault L, Miron M, Roussin A et al. Prospective Evaluation of Health-Related Quality of Life in Patients With Deep Venous Thrombosis. JAMA. 2005;165(10):1173-1178.

Kakkar A, Brenner B, Dahl O, Eriksson B, Mouret P, Muntz J et al. Extended duration rivaroxaban versus short-term enoxaparin for the prevention of venous thromboembolism after total hip arthroplasty: a double-blind, randomised controlled trial. Lancet. 2008;372(9632):31-39.

Kakkos S, Caprini J, Geroulako, G, Nicolaides A, Stansby G, Reddy D. Combined intermittent pneumatic leg compression and pharmacological prophylaxis for prevention of venous thromboembolism in high-risk patients (Cochrane Review). Cochrane Database Syst Rev. 2011;(3):CD005258.

Katzmarzyk T, Church S, Craig C, Bouchard C. Sitting time and mortality from all causes, cardiovascular disease, and cancer. Med Sci Sports Exerc.2009;41(5):998-1005.

Kesteven $\mathrm{P}$, Robinson B. Clinical risk factors for venous thrombosis associated with air travel. Aviat Space Environ Med. 2001;72(2):125-128.

Kock HJ, Schmit-Neuerburg KP, Hanke J, Rudofsky G, Hirche H. Thromboprophylaxis with lowmolecular-weight heparin in outpatients with plaster-cast immobilisation of the leg. Lancet. 1995;346(8973):459-461.

Kock H, Schmit-Neuerburg KP, Hanke J, Hakmann A, Althoff M, Rudofsky G, Hirche H. Ambulatory prevention of thrombosis with low molecular weight heparin in plaster immobilization of the lower extremity. Chirurg. 1993;64(6):483-91. [article in German].

Koster A, Caserotti P, Patel K, Matthews C, Berrigan D, van Domelen D et al. Association of Sedentary time with mortality independent of moderate to vigorous physical activity. PLOS ONE. 2012;7(6):e37696.

Kuipers S, Cannegieter S, Middeldorp S, Robyn L, Büller H, Rosendaal F. The absolute risk of venous thrombosis after air travel: A cohort study of 8,755 employees of international organisations. PLoS Medicine. 2007;4(9):e290.

Kuipers S, Venemans-Jellema A, Cannegieter SC, van Haften M, Middeldorp S, Buller HR, Rosendaal, FR. The incidence of venous thromboembolism in commercial airline pilots: a cohort study of 2630 pilots. J Thromb Haemost. 2014;12(8):1260-1265.

Kujath P, Spannagel U, Habscheid W. Incidence and prophylaxis of deep vein thrombosis in outpatients with injury of the lower limb. Haemostasis. 1993;23(suppl 1):20-26. 
Kujovich J. Factor V Leiden thrombophilia. Genet Med. 2011;13(1):1-16.

Lang I, Madan M. Update on chronic thromboembolic pulmonary hypertension. Circulation. 2014;130(6):508-518.

Lapidus L, Ponzer S, Elvin A, Levander C, Larfars G, Rosfors S, de Bri E. Prolonged thromboprophylaxis with Dalteparin during immobilization after ankle fracture surgery: a randomized placebo-controlled, double-blind study. Acta Orthop. 2007;78(4): 528-535.

Lapidus L, Rosfors S, Ponzer S, Levander C, Elvin A, Larfars G, de Bri E. Prolonged thromboprophylaxis with dalteparin after surgical treatment of achilles tendon rupture: a randomized, placebo-controlled study. J Orthop Trauma. 2007;21(1):52-57.

Lapidus L, Ponzer S, Pettersson H, de Bri E. Symptomatic venous thromboembolism and mortality in orthopaedic surgery - an observational study of 45968 consecutive procedures. BMC Musculoskelet Disord. 2013;14(1):177.

Lapostolle F, Surget V, Borron SW, Desmaizieres M, Sordelet D, Lapandry C et al. Severe pulmonary embolism associated with air travel. N Engl J Med. 2001;345(11):779-783.

Lassen M, Ageno W, Borris L, Lieberman J, Rosencher N, Bandel T, et al. Rivaroxaban versus Enoxaparin for Thromboprophylaxis after Total Knee Arthroplasty. N Engl J Med. 2008;358(26):2776-86.

Lassen MR, Borris LC, Nakov RL. Use of the low-molecular-weight heparin reviparin to prevent deepvein thrombosis after leg injury requiring immobilization. N Engl J Med. 2002;347(10):726-730.

Lee A, Levine M. Venous thromboembolism and cancer: Risks and outcomes. Circulation. 2003;107:117-121.

Lee $\mathrm{H}$. A new case of fatal pulmonary thromboembolism associated with prolonged sitting at computer in Korea. Yonsei Med J. 2004;45(2):349-351.

Lee K, Lim C, Hsu A. Letter to the Editor: Killed by Internet Addiction ? A Real Possibility. Annals Academy of Medicine, 2015;44(12):565-566.

Levin E, Macintosh D, Baker T, Weatherall M, Beasley R. Effect of sitting in ergonomic chairs on lower limb venous blood flow. Occ Erg. 2008;8(2):125-132.

Lidegaard O, Nielsen LH, Skovlund CW, Skjeldestad FE, Lokkegaard E. Risk of venous thromboembolism from use of oral contraceptives containing different progestogens and oestrogen doses: Danish cohort study, 2001-9. BMJ. 2011;343:d6423.

Llach F. Hypercoagulability, renal vein thrombosis, and other thrombotic complications of nephrotic syndrome: Editorial review. Kidney Int. 1985;28(3):429-439.

Lopez-Reyes R, Nauffal D, Ballester M, Martin-Antorian J, de Sousa M, Riera-Mestre A et al. Venous thromboembolism in patients immobilised at home. Eur Respir J. 2015;45:1728-1731. 
Macdonald R, Amidei C, Baron J, Weir B, Brown F, Erickson R et al. Randomized, pilot study of intermittent pneumatic compression devices plus dalteparin versus intermittent pneumatic compression devices plus heparin for prevention of venous thromboembolism in patients undergoing craniotomy. Surg Neurol. 2003;59(5):363.

MacMahon S, Rodgers A, Collins R, Farrell B. Antiplatelet therapy to prevent thrombosis after hip frature: rationale for a randomised trial. J Bone Joint Surg Br. 1994;76(4):521-524.

Makhdom AM, Cota A, Saran N, Chaytor R. Incidence of symptomatic deep venous thrombosis after Achilles tendon rupture. J Foot Ankle Surg. 2013;52(5):584-587.

Manly DA, Boles J, Mackman N. Role of tissue factor in venous thrombosis. Annu Rev Physiol. 2011;73:515-25.

Martinelli I, Taioli E, Battaglioli T, Podda G, Passamonti S, Pedotti P, Mannucci P. Risk of Venous Thromboembolism After Air Travel. Arch Intern Med. 2003;163:2771-2774.

Matthews CE, George SM, Moore SC, Bowles HR, Blair A, Park Y et al. Amount of time spent in sedentary behaviors and cause-specific mortality in US adults 1-3. Am I Clin Nutr. 2012;95(2):437-445.

Meek R, Tong RL-K. Venous thromboembolism in emergency department patients with rigid immobilization for lower leg injury: Incidence and risk factors. Emerg Med Australas. 2012;24(3):277-284.

Meissner MH, Caps MT, Bergelin RO, Manzo RA, Strandness DE. (1997). Early outcome after isolated calf vein thrombosis. J Vasc Surg. 1997;26(5):749-56.

Mercer A, Brown J. Venous thromboembolism associated with air travel: a report of 33 patients. Aviat Space Environ Med. 1998;69(2):154-157.

Mizel MS, Temple HT, Michelson JD, Alvarez RG, Clanton TO, Frey CC et al. Thromboembolism after foot and ankle surgery. A multicenter study. Clin Orthop Relat Res. 1998:(348):180-185.

Morita H, Abe C, Tanaka K, Shiratori M, Oguri M, Shiga T. Neuromuscular electrical stimulation and an ottoman-type seat effectively improve popliteal venous flow in a sitting position. J Physiol Sci. 2006;56(2):183-6.

Morris RJ, Woodcock JP, Pitto RP, Young S. Evidence-Based Compression: Prevention of stasis and deep vein thrombosis. Ann Surg. 2004;239(3):162-171.

Mummery WK, Schofield GM, Steele R, Eakin EG, Brown WJ. Occupational sitting time and overweight and obesity in Australian workers. Am J Prev Med. 2005;29(2):91-97.

Naide, M. (1952). Spontaneous venous thrombosis in the legs of tall men. JAMA. 148(14), 1202-3.

National Institute for Health and Clinical Excellence. (January 2010). Venous thromboembolism: reducing the risk for patients in hospital. Venous thromboembolism: reducing the risk for patients in hospital. 
National Institute of Clinical Studies. (2005). The Incidence and Risk Factors for Venous Thromboembolism in Hospitals in Western Australia 1999-2001. 2005. National Institute of Clinical Studies, Australia.

Nauffal D, Ballester M, Reyes RL, Jiménez D, Otero R, Quintavalla R, Monreal M. Influence of recent immobilization and recent surgery on mortality in patients with pulmonary embolism. $J$ Thromb Haemost. 2012;10(9):1752-1760

New Zealand Accident Compensation Corporation (ACC). (2013). Preventing and managing discomfort pain and injury.

New Zealand Venous Thromboembolism Prevention Steering Group. (2012). National Policy Framework: VTE Prevention in Adult Hospitalised Patients.

Ng SM., Khurana RM., Yeang HAW, Hughe, UM, Manning DJ. Is prolonged use of computer games a risk factor for deep venous thrombosis in children? Clin Med. 2003;3(6):593-4.

NHS. (2010). Prevention and management of venous thromboembolism: a national clinical guideline. Scottish Intercollegiate Guidelines Network.

Nickel K, Labberton L, Long A, Langer F, Fuchs T, Stavrou E et al. The polyphosphate/factor XII pathway in cancer-associated thrombosis: novel perspectives for safe anticoagulation in patients with malignancies. Thromb Res. 2016;141(suppl 2):S4-S7.

Nicolaides A, Miles C, Hoare M, Jury P, Helmis E, Venniker R. Intermittent sequential pneumatic compression of the legs and thromboembolism-deterrent stockings in the prevention of postoperative deep venous thrombosis. Surgery (UK). 1983;94(1):21-5.

Nilsson-Helander K, Thurin A, Karlsson J, Eriksson BI. High incidence of deep venous thrombosis after Achilles tendon rupture: a prospective study. Knee Surg Sports Traumatol Arthrosc. 2009;17(10):1234-1238.

Nordström M, Lindblad B, Bergqvist D, Kjellström T. (1992). A prospective study of the incidence of deep-vein thrombosis within a defined urban population. J Intern Med. 1992;232(2):155-60.

Oger E. Incidence of venous thromboembolism: a community-based study in Western France. EPIGETBP Study Group. Groupe d'Etude de la Thrombose de Bretagne Occidentale. Thromb Haemost. 2000;83(5):657-60.

Paganin F, Bourde A, Yvin JL., Genin R, Guijarro JL, Bourdin A, Lassalle C. Venous thromboembolism in passengers following a 12-h flight: a case control study. Aviat Space Environ Med. 2003;74(12):1277-1280.

Palareti, G., \& Sartori, M. (2016). Treatment of Isolated Below the Knee Deep Vein Thrombosis. Current Atherosclerosis Reports. 18(7), 37. http://doi.org/10.1007/s11883-016-0594-1

Patel A, Ogawa B, Charlton T, Thordarson D. Incidence of deep vein thrombosis and pulmonary embolism after Achilles tendon rupture. Clin Orthop Relat Res. 2012;470(1):270-274. 
Patel AV, Bernstein L, Deka A, Feigelson HS, Campbell PT, Gapstur SM et al. (2010). Leisure time spent sitting in relation to total mortality in a prospective cohort of US adults. Am J Epidemiol. 2010;172(4):419-429.

Patil S, Gandhi J, Curzon I, Hui ACW. Incidence of deep-vein thrombosis in patients with fractures of the ankle treated in a plaster cast. J Bone Joint Surg [Br]. 2007;89(10):1340-1343.

Perez-Rodriguez E, Jimenez D, Diaz G, Perez-Walton I, Luque M, Guillen C et al. Incidence of air travel-related pulmonary embolism at the Madrid-Barajas airport. Arch Intern Med. 2003;163(22):2766-2770.

Pitto RP, Young S. (2008). Foot pumps without graduated compression stockings for prevention of deep-vein thrombosis in total joint replacement: efficacy, safety and patient compliance. Int Orthop. 2008;32(3):331-336.

Planes A, Vochelle N, Mazas F, Mansat C, Zucman J, Landais A et al. Prevention of postoperative venous thrombosis: a randomized trial comparing unfractionated heparin with low molecular weight heparin in patients undergoing total hip replacement. Thromb Haemost. 1998;60(3):407-410.

Poelkens F, Thijssen DHJ, Kersten B, Scheurwater H, van Laarhoven EW, Hopman MTE. Counteracting venous stasis during acute lower leg immobilization. Acta Physiol. 2006;186(2):111-8.

Prandoni P, Lensing A, Cogo A, Cuppini S, Villalta S, Carta M et al. The long term clinical course of acute deep vein thrombosis of the arm: prospective cohort study. Ann Intern Med. 1996;125:17.

Printen K, Miller E, Mason E, Barnes R. Venous thromboembolism in the morbidly obese. Surg Gynecol Obstet. 1978;147(1):63-64.

Pulmonary Embolism Prevention (PEP) Trial Collaborative Group. Prevention of pulmonary embolism and deep vein thrombosis with low dose aspirin: Pulmonary Embolism Prevention (PEP) trial. Lancet. 2000;355(9212):1295-1302.

Qu H, Li Z, Zhai Z, Liu C, Wang S, Guo S, Zhang Z. Predicting of Venous Thromboembolism for Patients Undergoing Gynecological Surgery. Medicine. 2015;94(39):e1653.

Randelli F, Romanini E, Biggi F, Danelli G, Della Rocca G, Laurora NR et al. (2013). II Italian intersociety consensus statement on antithrombotic prophylaxis in orthopaedics and traumatology. J Orthopaed Traumatol. 2013;14(1):1-13.

Rebouças D, Costa M, Thuler L, Garces A, Aquino L, Bines J. Breast cancer-associated venous thromboembolism: A case-control study. Breast. 2016;28:84-88.

Reilmann $\mathrm{H}$, Weinberg A, Forster E, Happe B. Prevention of thombosis in ambulatory patients. Der Orthpade. 1993;22(2):117 - 120. [Article in German]. 
Riou B, Rothmann C, Lecoules N, Bouvat E, Bosson JL, Ravaud P et al. Incidence and risk factors for venous thromboembolism in patients with nonsurgical isolated lower limb injuries. Am J Emerg Med. 2007;25(5):502-508.

Roberts C, Horner D, Coleman G, Maitland L. Curl-Roper T, Smith R et al. Guidelines in Emergency Medicine Network (GEMNet): guideline for the use of thromboprophylaxis in ambulatory trauma patients requiring temporary limb immobilisation. Emerg Med J. 2013;30(11):968-982.

Rockette-Wagner B, Edelstein S, Venditti E, Reddy D, Bra, G, Carrion-Petersen M, For The Diabetes Prevention Program Research Group. The impact of lifestyle intervention on sedentary time in individuals at high risk of diabetes. Diabetologia. 2015;58;1198-1202.

Rosendaal F. Venous Thrombosis: The Role of Genes, Environment, and Behavior. Hematology Am Soc Hematol Educ Program. 2005;(1):1-12.

Salazar C, Malaga G, Malasquez G. Direct thrombin inhibitors versus vitamin K antagonists or low molecular weight heparins for prevention of venous thromboembolism following total hip or knee replacement: Cochrane Review. Cochrane Database Syst Rev. 2010;(4):CD005981.

Samama MM. An epidemiologic study of risk factors for deep vein thrombosis in medical outpatients: the Sirius study. Arch Intern Med. 2011;160(22):3415-3420.

Sanfilippo K, Wang T, Gage B, Luo S, Riedell P, Carson K. Incidence of venous thromboembolism in patients with non-Hodgkin lymphoma. Thromb Res. 2016;143:86-90.

Santori F, Vitullo A, Stopponi M, Santori N, Ghera S. Prophylaxis against deep-vein thrombosis in total hip replacement: Comparison of heparin and foot impulse pump. J Bone Joint Surg $[B r]$. 1994;76-B(4):579-583.

Saragas N, \& Ferrao P. The incidence of venous thromboembolism in patients undergoing surgery for acute Achilles tendon ruptures. Foot Ankle Surg. 2011;17(4):263-5.

Sarvesvaran R. Sudden natural deaths associated with commercial air travel. Med Sci Law. 1986;26(1):35-8.

Schmid D, Leitzmann MF. Television viewing and time spent sedentary in relation to cancer risk: A meta-analysis. J Natl Cancer Inst. 2014;106(7):1-19.

Schobersberger W, Fries D, Mittermayr M, Innerhofer P, Sumann G, Schobersberger B et al. Changes of biochemical markers and functional tests for clot formation during long-haul flights. Thromb Res. 2003;108(1):19-24.

Schobersberger W, Mittermayr M, Innerhofer P, Sumann G, Schobersberger B, Klingler A et al. Coagulation changes and edema formation during long-distance bus travel. Blood Coagul Fibrinolysis. 2004;15(5):419-425.

Schwarz T, Siegert G, Oettler W, Halbritter K, Beyer J, Frommhold R et al. Venous thrombosis after long-haul flights. Arch Intern Med. 2003;163(22):2759-64. 
Selby R, Geerts WH, Krede, HJ, Crowther MA, Kaus L, Sealey F. Symptomatic venous thromboembolism uncommon without thromboprophylaxis after isolated lower-limb fracture: the knee-to-ankle fracture (KAF) cohort study. J Bone Joint Surg Am. 2014;96(10):e83.

Selby R, Geert, W, Kreder H, Crowther M, Kaus L, Sealey F. A double-blind, randomized controlled trial of the prevention of clinically important venous thromboembolism after isolated lower leg fractures. J Orthop Trauma. 2015;29(5):224-30.

Sevitt S, Gallagher N. Venous thrombosis and pulmonary embolism. A clinico-pathological study in injured and burned patients. Br J Surg. 1961;48(211):475-89.

Sharpe R, Gupta R, Gracias V, Pryor J, Pieracci F, Reilly P, Schwab C. Incidence and natural history of below-knee deep venous thrombosis in high-risk trauma patients. J Trauma. 2002;53(6):104852.

Shibuya N, Frost CH, Campbell JD, Davis ML, Jupiter DC. Incidence of acute deep vein thrombosis and pulmonary embolism in foot and ankle trauma: analysis of the National Trauma Data Bank. J Foot Ankle Surg. 2012;51(1):63-68.

Silverstein MD, Heit JA, Mohr DN, Petterson TM, O'Fallon WM, Melton III LJ. Trends in the incidence of deep vein thrombosis and pulmonary embolism: a 25 -year population-based study. Arch Intern Med. 1998;158(6):585-593.

Simpson K. Shelter deaths from pulmonary embolism. Lancet. 1940;2(6120):744.

Sitthipornvorakul E, Janwantanakul P, van der Beek A J. Correlation between pedometer and the Global Physical Activity Questionnaire on physical activity measurement in office workers. BMC Research Notes. 2014;7(1):280.

Solis G, Saxby T. Incidence of DVT following surgery of the foot and ankle. Foot Ankle Int. 2002;23(5):411-4.

Sonnevi K. (2013). Venous thromboembolism in women: risk factors and long term follow-up. Karolinska Institutet, Stockholm, Sweden.

SooHoo N, Krenek L, Eagan M, Gurbani B, Ko C, Zingmond D. Complication Rates Following Open Reduction and Internal Fixation of Ankle Fractures. J Bone Joint Surg Am. 2009;91(5):10421049.

Spannagel U, Kujath P. Low molecular weight heparin for the prevention of thromboembolism in outpatients immobilized by plaster cast. Semin Thromb Haemost. 1993;19(Suppl 1):131-141.

Spyropoulos A, Lin J. Direct medical costs of venous thromboembolism and subsequent hospital readmission rates: an administrative claims analysis from 30 managed care organizations. $J$ Manag Care Pharm. 2007;13(6):475-486

Statistics New Zealand. (2016). Statistics New Zealand: Household Labour Force Survey tables for March 2016 quarter. 
Stein P, Matta F. Epidemiology and incidence: The scope of the problem and risk factors for development of venous thromboembolism. Clin Chest Med. 2010;31(4): 611-628.

Straker L, Levine J, Campbell A. The Effects of Walking and Cycling Computer Workstations on Keyboard and Mouse Performance. Hum Factors. 2009;51(6):831-844.

Suadicani P, Hannerz H, Bach E, Gyntelberg F. Jobs encompassing prolonged sitting in cramped positions and risk of venous thromboembolism: cohort study. J R Soc Med Sh Rep. 2012;3(2):8.

Sweetland, S., Green, J., Liu, B., Berrington de Gonzalez, A., Canonico, M., Reeves, G., \& Beral, V. (2009). Duration and magnitude of the postoperative risk of venous thromboembolism in middle aged women: prospective cohort study. BMJ. 339(1):b4583. http://doi.org/10.1136/bmj.b4583

Symington I, Stack B. Pulmonary thromboembolism after travel. Br J Dis Chest. 1997;71(2):138-40.

Testroote M, Stigter WA, de Visser DC, Janzing HM, Stigter-Willem AH, Janssen L et al. Low molecular weight heparin for prevention of venous thromboembolism in patients with lower-leg immobilization. (Cochrane Review). Cochrane Database Syst Rev. 2014;(3):CD006681.

Testroote M, Stigter W, de Visser D, Janzing H. Low molecular weight heparin for prevention of venous thromboembolism in patients with lower-leg immobilization (Cochrane Review). Cochrane Database Syst Rev. 2008;(3):CD006681.

Thompson WG, Foster RC, Eide DS, Levine JA. Feasibility of a walking workstation to increase daily walking. Br. J Sports Med. 2008;42(3):225-228.

Thordarson DB, Ghalambor N, Perlman M. Intermittent pneumatic pedal compression and edema resolution after acute ankle fracture: a prospective, randomized study. Foot Ankle Int. 1997;18(6):347-350.

Timsit J, Farkas J, Boyer J, Martin J, Misset B, Renaud B, Carlet J. Central vein catheter-related thrombosis in intensive care patients: Incidence, risks factors, and relationship with catheterrelated sepsis. Chest. 1998;114(1):207-213.

Tsai A, Cushman M, Rosamond W, Heckbert S, Polak J, Folsom A. Cardiovascular Risk Factors and Venous Thromboembolism Incidence The Longitudinal Investigation of Thromboembolism Etiology. Arch Intern Med. 2002;162(10):1182-9.

Turpie A, Bauer K, Caprini J, Comp P, Gent M, Muntz J. Fondaparinux combined with intermittent pneumatic compression vs. intermittent pneumatic compression alone for prevention of venous thromboembolism after abdominal surgery: a randomized, double-blind comparison. $J$ Thromb Haemost. 2007;5(9):1854-61.

Turpie A, Levine M, Hirsh J, Carter C, Jay R, Powers P et al. A randomized controlled trial of a lowmolecular-weight heparin (enoxaparin) to prevent deep-vein thrombosis in patients undergoing elective hip surgery. N Eng/ J Med. 1986;315(15):925-9. 
VADOplex. (2016). VADOplex. Retrieved July 25, 2016, from http://www.vadoplex.com/deen/application-under-cast.html

Van Adrichem R, Debeij J, Nelissen R, Schipper I, Rosendaal F, Cannegieter S. Below-knee cast immobilization and the risk of venous thrombosis: results from a large population-based casecontrol study. J Thromb Haemost. 2014;12:1461-9.

Van der Ploeg H, Chey T, Korda R, Banks E, Bauman A. Sitting Time and All-Cause Mortality Risk in 222497 Australian Adults. Arch Intern Med. 2012;172(6):494-500.

Van Stralen K, Rosendaal F, Doggen C. Minor injuries as a risk factor for venous thrombosis. Arch Intern Med. 2008;168(1):21-26.

Venkatachalam S, Wright KU. Fatal pulmonary embolism following conservatively managed tendo achilles rupture-A case report. Int J Surg Case Rep. 2012;3(1):3-5.

Virchow R. (1856). Thrombose und Embolie. Gefässentzündung und septische Infektion. (A. Matzdorff \& W. Bell, Eds.) (Klassiker). Eingeleitet von Rudolf Beneke. Leipzig: reprint 1910. [Article in German].

Virchow R. (1859). Die Cellularpathologie in ihrer Begrundung auf physiologische und pathologische Gewebelehre. (Vol. Berling). Verlag von August Hirschwald. [Article in German].

Vollan S, Chaturvedi A, Sivasankaran K, Madhu T, Hadland Y, Allgar V, Sharma HK. Symptomatic venous thromboembolism following circular frame treatment for tibial fractures. Injury. 2015;46(6):1108-1111.

Walker A, Baldwin D, Card T, Powell H, Hubbard R, Grainge M. Risk of venous thromboembolism in people with lung cancer: a cohort study using linked UK healthcare data. Br J Cancer. 2016;115(1):115-21.

Warwick DJ, Shaikh A, Gadola SD, Stokes M, Worsley P, Bain D et al. Neuromuscular electrostimulation via the common peroneal nerve promotes lower limb blood flow in a belowknee cast: a potential for thromboprophylaxis. Bone Joint Res. 2013;2(9):179-185.

Waters C, Ling E, Chu A, Ng S, Chia A, Lim Y, Müller-Riemenschneider F. Assessing and understanding sedentary behaviour in office-based working adults: a mixed-method approach. BMC Public Health. 2016;16(1):360.

West J, Perrin K, Aldington S, Weatherall M, Beasley R. A case-control study of seated immobility at work as a risk factor for venous thromboembolism. J R Soc Med. 2008;101(5):237-243.

Westrich G, Bottner F, Windsor R, Laskin R, Haas S, Sculco T. VenaFlow Plus Lovenox vs VenaFlow Plus Aspirin for Thromboembolic Disease Prophylaxis in Total Knee Arthroplasty. J Arthroplasty. 2006;21(6 SUPPL 2):139-143.

Westrich G H, Haas S B, Mosca P, Peterson M. Meta-analysis of thromboembolic prophylaxis after total knee arthroplasty. J Bone Joint Surg [Br]. 2000;82-B(6):795-800. 
White R, Zhou H, Romano P. Incidence of symptomatic venous thromboembolism after different elective or urgent surgical procedures. Thromb Haemost. 2003;90:446-55.

Willenberg T, Schumacher A, Amann-Vesti B, Jacomella V, Thalhammer C, Diehm N et al. Impact of obesity on venous hemodynamics of the lower limbs. J Vasc Surg. 2010;52(3):664-8.

Wilson N, Das S, Maurice H, Smibert G, Thomas E, Kakkar V. Thrombosis prophylaxis in total knee replacement: does a simple mechanical method help? Br J Surg. 1990;77(12):1417.

Won S, Lee Y, Suh Y, Koo K. Extensive bullous complication associated with intermittent pneumatic compression. Yonsei Med J. 2013;54(3):801-802.

World Health Organisation (WHO). (2013). Global action plan for the prevention and control of noncommunicable diseases 2013-2020.

Wright HP, Osborn SB. Effect of posture on venous velocity, measured with $24 \mathrm{NaCl}$. Br Heart J. 1952;14(3):325-330.

Wukich DK, Waters D H. Thromboembolism following foot and ankle surgery: a case series and literature review. J Foot Ankle Surg. 2008;47(3):243-249. 